\title{
Health technology assessment in epilepsy
}

\author{
Citation for published version (APA):
}

Wijnen, B. F. M. (2017). Health technology assessment in epilepsy: moving towards patient-centered, efficient care. [Doctoral Thesis, Maastricht University]. Universitaire Pers Maastricht. https://doi.org/10.26481/dis.20170705bw

Document status and date:

Published: 01/01/2017

DOI:

10.26481/dis.20170705bw

Document Version:

Publisher's PDF, also known as Version of record

\section{Please check the document version of this publication:}

- A submitted manuscript is the version of the article upon submission and before peer-review. There can be important differences between the submitted version and the official published version of record.

People interested in the research are advised to contact the author for the final version of the publication, or visit the DOI to the publisher's website.

- The final author version and the galley proof are versions of the publication after peer review.

- The final published version features the final layout of the paper including the volume, issue and page numbers.

Link to publication

\footnotetext{
General rights rights.

- You may freely distribute the URL identifying the publication in the public portal. please follow below link for the End User Agreement:

www.umlib.nl/taverne-license

Take down policy

If you believe that this document breaches copyright please contact us at:

repository@maastrichtuniversity.nl

providing details and we will investigate your claim.
}

Copyright and moral rights for the publications made accessible in the public portal are retained by the authors and/or other copyright owners and it is a condition of accessing publications that users recognise and abide by the legal requirements associated with these

- Users may download and print one copy of any publication from the public portal for the purpose of private study or research.

- You may not further distribute the material or use it for any profit-making activity or commercial gain

If the publication is distributed under the terms of Article $25 \mathrm{fa}$ of the Dutch Copyright Act, indicated by the "Taverne" license above, 


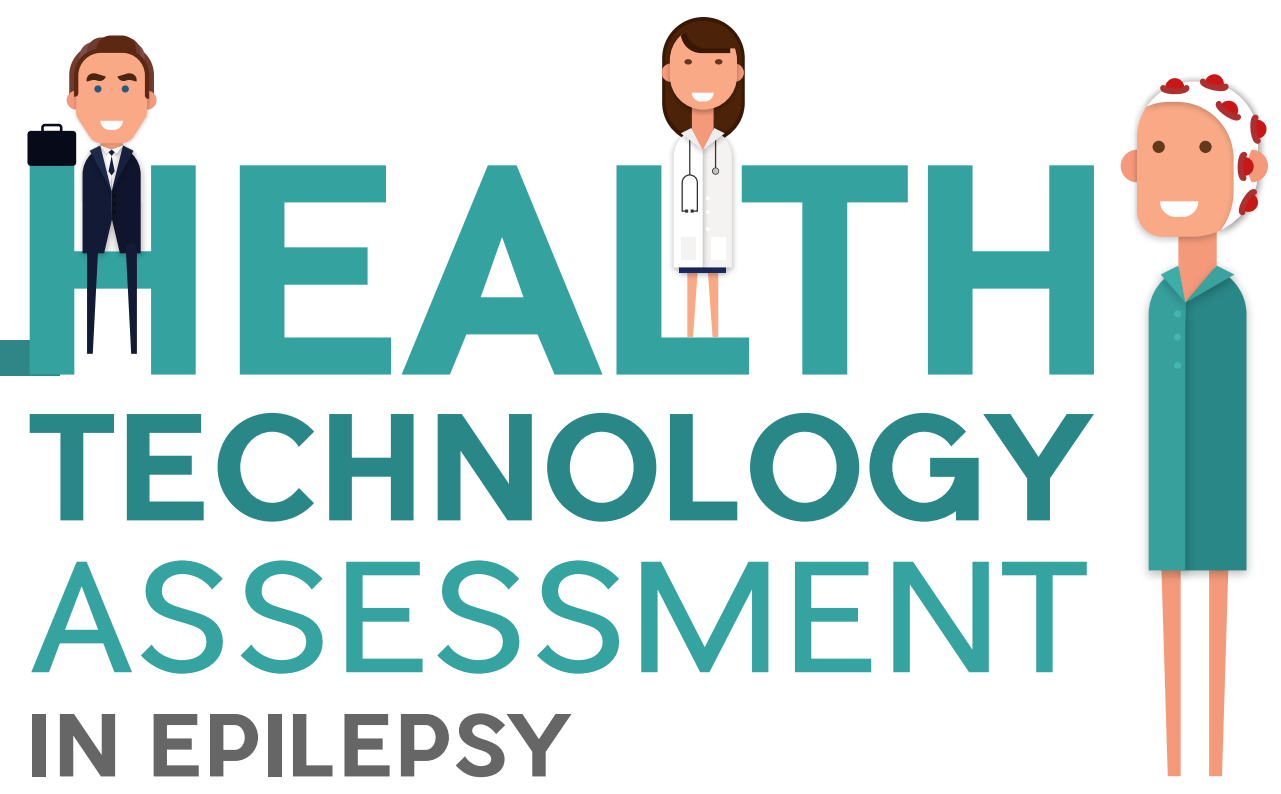

moving towards patient-centered, efficient care

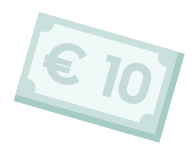

Ben F.M. Wijnen 
The studies presented in this thesis were conducted at the epilepsy center Kempenhaeghe and at the Care and Public Health Research Institute (CAPHRI), Department of Health Services Research (HSR) at Maastricht University.

The research presented in this thesis was funded by the Netherlands Organization for Health Research and Development (ZonMw; project171002204 [KOEK-study] and project 836011018 [ZMILE-study]).

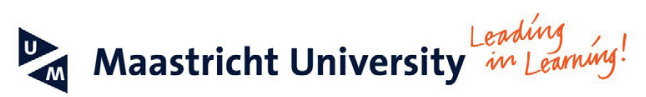

Kempenhaeghe
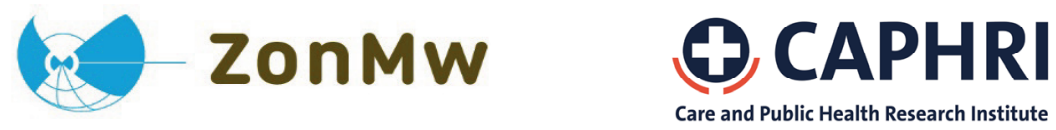

Printing and dissemination of this thesis was financially supported by CAPHRI HTA program, Kempenhaeghe, Nutricia Advanced Medical Nutrition, and UCB Pharma B.V.

\section{Inspired by patients. Driven by science.}

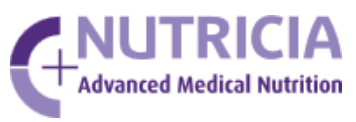

(c) Ben Wijnen, Maastricht 2017

No part of this book may be reproduced or transmitted in any form or by any means, without prior permission in writing by the author, or when appropriate, by the publishers of the publications.

Layout: Tiny Wouters

Cover design: Kim Deuning

Printing: Datawyse | Universitaire Pers Maastricht

ISBN: 9789461597021

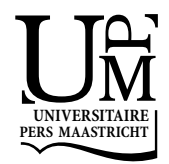




\title{
Health technology assessment in epilepsy; moving towards patient-centered, efficient care
}

\author{
PROEFSCHRIFT \\ ter verkrijging van de graad van doctor aan de Universiteit Maastricht, \\ op gezag van de Rector Magnificus, Prof. dr. Rianne M. Letschert, \\ volgens het besluit van het College van Decanen, \\ in het openbaar te verdedigen \\ op woensdag 5 juli 2017 om 14:00 uur
}

door

Ben Franciscus Martinus Wiinen 


\section{Promotores}

Prof. dr. mr. S.M.A.A. Evers

Prof. dr. H.J.M. Majoie

\section{Co-promotor}

Dr. R.J.A. de Kinderen

\section{Beoordelingscommissie}

Prof. dr. M.A. Joore (voorzitter)

Prof. dr. P.A.J.M. Boon (Academisch Centrum voor Epilepsie Kempenhaeghe \& Universitair Ziekenhuis Gent)

Dr. J. Lokkerbol (Trimbos Instituut Utrecht)

Dr. J. Nicolai

Prof. dr. D. Ruwaard 



\section{Contents}

Chapter 1: General introduction

Part I Methodological aspects and preference based studies in the health technology assessment of epilepsy

Chapter 2: Eliciting patients' preferences for epilepsy diagnostics:

A discrete choice experiment.

Chapter 3: From clinically relevant outcome measures to quality of life in epilepsy: A time trade-off study

Chapter 4: $\quad$ Mapping of the QOLIE-3 IP to EQ-5D-5L utilities and comparison of the responsiveness of both instruments in epilepsy

Chapter 5: Using best-worst scaling to investigate preferences in health care: A systematic review

Part II Economic evaluations within epilepsy

Chapter 6: How to prepare a systematic review of economic evaluations for informing evidence-based healthcare decisions: data extraction, risk of bias, and transferability (Part 3 of 3)

Chapter 7: A systematic review of economic evaluations of treatments for patients with epilepsy

Chapter 8: Long-term clinical outcomes and economic evaluation of the ketogenic diet versus care as usual among children and adolescents with intractable epilepsy

Chapter 9: (Cost)-effectiveness of a multi-component intervention for adults with epilepsy: study protocol of a Dutch randomized controlled trial (ZMILE study).

Chapter 10: An economic evaluation of a multi-component Intervention for adults with epilepsy (ZMILE study) 
Part III Discussion and summary

Chapter 11: General discussion 223

$\begin{array}{lll}\text { Chapter 12: } & \text { Summary } & 247\end{array}$

$\begin{array}{ll}\text { Chapter 13: } & \text { Samenvatting } 253\end{array}$

Part IV Addendum

$\begin{array}{ll}\text { Valorization } & 261\end{array}$

$\begin{array}{ll}\text { List of publications } & 267\end{array}$

$\begin{array}{ll}\text { Dankwoord } & 273\end{array}$

$\begin{array}{ll}\text { Curriculum vitae } & 281\end{array}$ 



\section{General introduction to the}

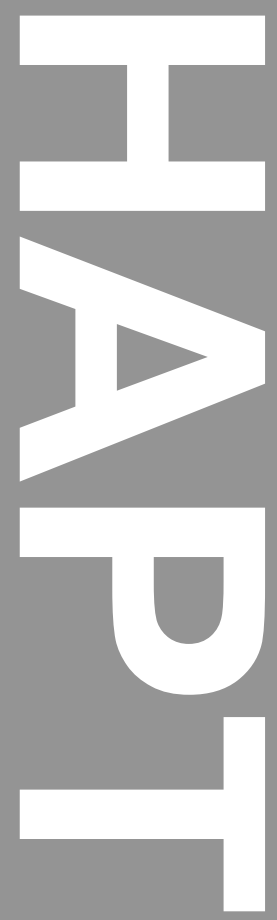

thesis
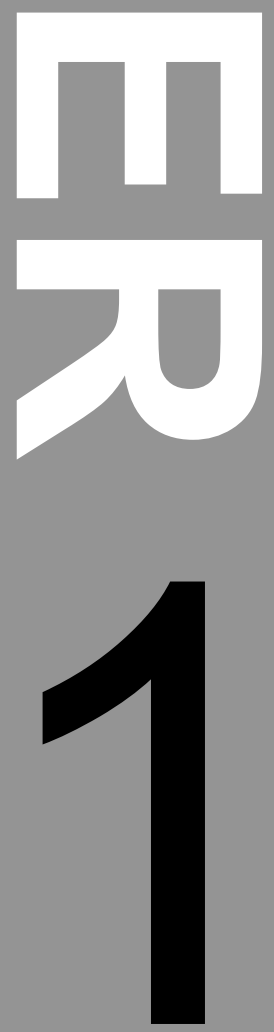

Parts of the general introduction have been published as:

Wijnen, B.F.M., Majoie, M.H.J.M., Leenen, L.A.M., \& Evers, S.M.A.A. (2015). Economische evaluatiestudies binnen de epilepsie. Epilepsie, periodiek voor professionals, 13/2), 16-20 



\section{Burden of epilepsy}

Epilepsy is a neurological disorder, characterized by recurrent unprovoked seizures. A seizure is the result of sudden, usually brief, excessive electrical discharges in a group of nerve cells in the brain. Different parts of the brain can be the site of such discharges. Clinical manifestations of seizures will, therefore, vary depending on where in the brain the disturbance first starts and how far it spreads. Transient symptoms, such as loss of awareness or consciousness and disturbances of movement, sensation, mood or mental function can occur. Two major categories of seizure can be distinguished: generalized seizures (electrical discharge involves the whole brain) and partial seizures (electrical discharge involves one or more localized areas of the brain). ${ }^{\prime}$ In the Netherlands, approximately 120,000 people are currently suffering from epilepsy; the incidence is estimated at 30 per 100,000 people, meaning there are 5,000 to 8,000 newly identified cases per year. ${ }^{2}$ In 2015 , based on the Netherlands Institute for Health Services Research (NIVEL) Primary Care Database, the prevalence of epilepsy in the Netherlands was even estimated to be 180,000 with an incidence of 14,600 new cases. ${ }^{3}$

Epilepsy has a large impact on patients' quality of life, ${ }^{4,5}$ their surroundings and society as a whole. ${ }^{6,7}$ A study which examined DALYs (disability-adjusted life years) suggested that globally, around $1 \%$ of the number of days lost due to ill health may be due to epilepsy. ${ }^{8}$ The same study estimated that the aggregate burden due to epilepsy is around $0.5 \%$ of the total disease burden. ${ }^{8}$ This condition also has a considerable effect on the patient's psychological and emotional state. In the Netherlands, children with epilepsy seem to have poorer school attendance and performance. ${ }^{9}$ Moreover, adults with epilepsy are twice as likely to self-report depression or anxiety. Adults with active epilepsy were 3 times as likely to self-report depression and twice as likely to have anxiety compared to adults without epilepsy. ${ }^{10}$ It has been argued that depression affects $20-50 \%$ of individuals with epilepsy. ${ }^{11}$ Lastly, it has been estimated that up to $50 \%$ of epilepsy cases have cognitive impairments that affect health, productivity and quality of life. ${ }^{12}$

\subsection{Clinical trajectory}

In current practice, there is still a considerable need for improvement of the diagnosis, classification, treatment and management of epilepsy. For example, diagnosing epilepsy is a lengthy process that can be a burden for the patient and weighs (financially) heavily on the health system (health insurance), while misdiagnoses are quite common. As seizures are infrequent events in the majority of patients, recording of ictal (e.g. during seizures) EEG is both time-consuming and expensive. The mainstay of diagnosis, therefore, remains the detection of interictal epileptiform discharges (IEDs). ${ }^{13}$ IEDs in the electroencephalograph are used to confirm the clinical diagnosis of epilepsy and to classify the epilepsy syndrome in patients. ${ }^{14}$

About $30 \%$ of the patients with epilepsy are not seizure-free while on anti-epileptic drugs (AEDs). These patients, with uncontrolled epileptic seizures and in whom two or more 
AEDs have failed, are commonly referred to as having refractory or intractable epilepsy. Patients with intractable epilepsy, depending on their preferences and comorbidities, may be eligible for several other treatments such as vagus nerve stimulation, deep brain stimulation, the ketogenic diet and surgery depending on the type and localization of the epilepsy.

\subsection{Economic impact}

Although the impact of interventions on population health is vital, it is also important to determine the role of different interventions in contributing to other socially desirable goals, such as improving patients' quality of life and reducing societal costs. Societal costs include healthcare and other (inter-sectoral) costs such as productivity losses. In addition, due to scarcity of resources and rising costs in the Dutch healthcare system, economic evaluations are becoming more important for decision-makers and healthcare reimbursement companies. In 2011, in The Netherlands, costs of epilepsy were about 248.7 million euro, that is $4.8 \%$ of the total healthcare costs spent on neurological conditions. ${ }^{15}$ This is comparable to the cost of epilepsy in other western countries. ${ }^{6,7}$ Furthermore, healthcare costs attributable to epilepsy are rising, especially in the past decades, which highlights the need for an efficient allocation of resources (see Figure 1.1).

The costs presented in Figure 1.1 reflect healthcare costs, which only represent a small part of the costs attributable to epilepsy. For example, various studies have found that the unemployment rate among epileptic patients is at least twice as high as in the general population; when epileptic patients do find a job, they find it difficult to hold onto it, leading to even higher rates of unemployment. ${ }^{16-18}$ In addition, living with epilepsy may lead to dependency on others (e.g. for transport or housekeeping).

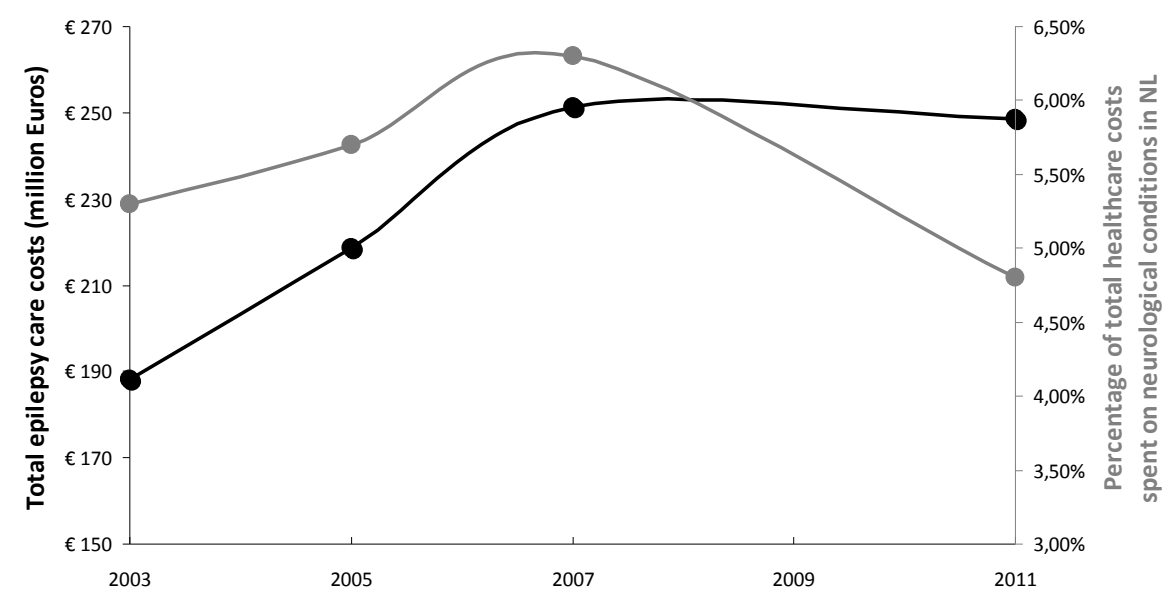

Figure 1.1 Overview of healthcare expenditure in the Netherlands attributable to epilepsy (source: www.kostenvanziekten.nl). 


\section{The field of health technology assessment (HTA)}

Health technology assessment is a broad multidisciplinary field which examines the social, economic, organizational and ethical issues of a health intervention or health technology. ${ }^{19}$ HTA aims to provide policymakers and practitioners with evidence-based choices in health services and patient care. This thesis will focus on two disciplines in particular, namely research into patient preferences and economic evaluations.

\subsection{Patient preferences}

In HTA, patient preferences form an important factor in determining the overall quality of a (new) treatment or clinical tool. Eliciting preferences has become increasingly important in healthcare ${ }^{20}$; understanding them can be very useful for both policy and clinical decisions. Over the years, weighing the patient's opinion in these decisions has become more evident $^{2}$; incorporating them in clinical decisions might also lead to improved adherence and outcomes. ${ }^{21}$ The most common way to measure preferences in healthcare is by stated preference (SP) methods, which involve the elicitation of responses to predefined alternatives, people hypothetically stating their preference. This is in contrast to revealed preference methods which involve observing preferences in real life. Three broad categories of SP methods have been distinguished: ranking, ratings and choice-based approaches. ${ }^{22}$ In ranking exercises, respondents will be given a pre-specified number of alternatives which they will be asked to rank from 'most preferable' to 'least preferable'. In rating scale exercises, respondents are usually asked to rate each alternative on a Likert scale with a pre-specified range (i.e. 1 to 7). The third category is a choice-based approach, for example a discrete choice experiment (DCE), involving the presentation of a series of pairwise choice tasks involving hypothetical scenarios, in which respondents are asked to select their preferred scenario. Another type of choice-based approach, namely best-worst scaling (BWS), is becoming increasingly popular. ${ }^{23}$ BWS devised by Finn and Louviere $(1992)^{24}$ and formally introduced to healthcare research by Mclntosh and Louviere (2002), ${ }^{25}$ is a novel method in which respondents are asked to indicate the best and the worst in a set of available items or options.

\begin{tabular}{|l|l|l|}
\hline Characteristics & Method A & Method B \\
\hline Way of measuring brain activity & By use of a helmet & By use of an electro cap \\
\hline Duration of recording & 30 minutes & 90 minutes \\
\hline Freedom of movement & No movements possible & Minor movements possible \\
\hline Travel time & 30 minutes & 90 minutes \\
\hline Type of additional examination & EEG after sleep deprivation & 24-hour EEG \\
\hline Chance of additional examination & $50 \%$ (50 out of 100 patients) & $20 \%$ (20 out of 100 patients) \\
\hline Which option do you prefer? & $\square$ & \\
\hline
\end{tabular}

Figure 1.2 Example of a DCE choice set (adapted from Wiinen et al. ${ }^{26}$ ). 
Preference studies are also used to determine the value people attach to certain health states. In health economics, the value of a health state (often referred to as a utility) is used to calculate quality adjusted life years (QALYs). A utility can be seen as measure which states the value of a particular health state. Utilities are expressed on a scale from 0 to 1 , in which 0 means "worst imaginable health state" and 1 means "perfect health". Next, utilities are used to calculate quality adjusted life years (QALYs), by multiplying the utility of a health state by the time spent in that particular health state. For example, if someone would live 5 years longer with a utility of 0.8 , then this person gains 4 QALYs $\left(5^{*} 0.8=4\right)$. Utilities can be determined in many ways but, if all else is equal, most health economists prefer choice-based methods to rating methods, as choosing, unlike rating, is a (more) natural human task at which we all have considerable experience; furthermore, it is observable and verifiable. ${ }^{27}$

As mentioned above, utilities are determined (or measured) for specific health states. Hence, if one knows the health state of a patient, one can easily calculate QALYs. Quality of life instruments, such as the EuroQol-5 dimensions-5 levels (EQ-5D-5L) or the ShortForm-36, are questionnaires which try to identify the health state (and hence quality of life) of patients by asking pre-specified questions with pre-specified response alternatives. For example, the EuroQol 5 Dimensions with 5 levels (EQ-5D-5L), consists of 5 dimensions: mobility, self-care, usual activities, pain/discomfort and anxiety/depression, and comprises 5 levels (ranging from no problems to extreme problems). Thus, a total of 3125 possible health states can be composed. If one knows the utilities associated with each health state, it is easy to determine respondents' utility by looking at their answers. The set of utilities corresponding to health states is often referred to as a valuation set. This is where preferences studies come in. In most cases, the general population is asked to put a utility value on each health state, often in an indirect manner. One way of doing this is to use the time-trade-off method (TTO), which is a choice-based preference approach. People are asked how many healthy years they are willing to give up in order to become indifferent between the specific disease state and the remaining years of life in perfect health. For example, if a person is told to live 10 years in health state $A$, and indicates that he or she is willing to give up 2 years of those 10 years to be in perfect health (i.e. 8 perfect years instead of 10 years in health state $A$ ), the corresponding utility for that health state is $(10-2) / 10=0.80$. By repeating this exercise for every health state and with a large enough sample, the valuation set is obtained.

Although patients' quality of life or utility is assessed by use of instruments such as the EQ-5D-5L, it is important to note that the general population is used to derive utility values, not the patients, the main reason being that it is often assumed that the use of patients' preferences may lead to underestimation due to adaptation to their health state. The general population is assumed to be best able to discriminate between diseases and conditions. 


\subsection{Economic evaluations}

In an economic evaluation (EE), one compares both the costs and effects of two (or more) alternative treatments or interventions in a systematic manner. Hence, it is possible to examine which alternative is most efficient. On the whole, there are two forms of EE: 1) an EE piggy-backed to a randomized controlled trial in which all costs and effects are measured (prospectively) during the trial period, a so-called trial-based EE, and 2) an EE based on previously published literature synthesized in a decision model, a so-called model-based EE. Model-based EE are generally used to examine long-term consequences, whereas trial-based EE are often used for short-term goals.

\subsubsection{Outcome measures}

The most common types of economic evaluations are the cost-effectiveness analysis (CEA) and the cost-utility analysis (CUA). A CEA is an economic evaluation in which the outcomes are expressed in natural units, such as seizure frequency or seizure severity. Hence, outcomes are often assessed with instruments used in clinical practice (e.g. blood pressure monitoring or EEG registration).

In a CUA, the outcomes are expressed in terms of quality of life. Quality of life is referred to as an individual's well-being including more subjective domains such as psychological and social state and not merely the absence of disease. As mentioned above, Quality of life is usually expressed in terms of utilities, which in turn can be used to calculate QALYs.

Often a distinction is made between disease-specific quality of life instruments, designed to be relevant to a particular condition or state, in this case epilepsy, ${ }^{28}$ and generic quality of life instruments. The latter are designed to be applicable across all diseases or conditions, across different medical interventions and across a wide range of populations.

In epilepsy, an often used disease-specific quality of life instrument is the Quality of Life in people living with epilepsy (QOLIE-31p). ${ }^{29}$ An example of a generic quality of life instrument is the EQ-5D-5L or the SF-36. Generic QALYs can be particularly useful for decision-makers as most instruments used to measure utilities are designed to be applicable in a large variety of conditions (generic quality of life instruments). Hence these outcomes can be used for reimbursement or policy decisions when deciding between several competing treatments covering a range of conditions.

\subsubsection{Categorization of costs}

To present all relevant and identified costs in an economic evaluation, the following categorization is often used $^{27}$ :

- Healthcare costs: these include treatment costs and all other medical costs incurred during the study follow-up, such as prevention, diagnostics, therapy and rehabilitation costs. 
- Patient \& family costs: these are all services paid by the patient, e.g. time invested, informal care and travel expenses.

- Productivity costs: costs caused by the (temporary) inability to perform professional activities or due to reduced productivity.

- Other costs: all costs in other (inter-sectoral) sectors, such as special education or housing adjustments (see Drost et al. $2014^{30}$ ).

Resource use is often measured by costs diaries or retrospective costs questionnaires. Questionnaires or diaries are especially important for measuring costs outside the healthcare sector as there are no databases available which contain this type information. For a more standardized approach to the valuation for identified costs, the Dutch Healthcare Institute has published the Guideline for Cost Research in the Netherlands; this includes standardized cost prices for the most common healthcare resources. ${ }^{31}$

\subsubsection{The cost-effectiveness criteria}

To value the expediency, or cost-effectiveness, of a certain intervention or treatment, it is essential to calculate the incremental cost-effectiveness ratio (ICER). This ratio combines both the costs and effects of two treatments into a single measure using the following formula:

$$
I C E R=\frac{\left(\text { Costs }_{\text {intervention group }}-\text { Costs }_{\text {control group }}\right)}{\left(E f f e c t s_{\text {intervention group }}-E f f e c t s_{\text {control group }}\right)}
$$

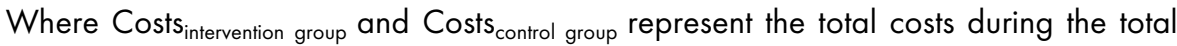

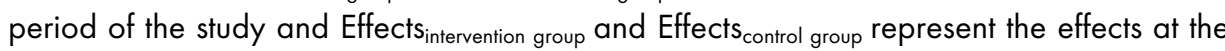
end of the follow-up. Using this formula, one can calculate the extra costs of one unit of effect. In case of a CUA, this will be the costs per one additional QALY gained.

For easy interpretation of the ICER, it is important to plot it in a cost-effectiveness plane (CE-plane). This is a graph in which the incremental effects are shown on the $x$-axis and the incremental costs on the $y$-axis. The slope of the point estimate (from the origin of the graph) represents the ICER. The CE-plane can be divided into four quadrants (see Figure 1.3). An ICER in quadrant $A$ represents lower effects combined with higher costs; hence the intervention is dominated by the comparator. An ICER in quadrant $D$ is associated with more effects and lower costs; hence the intervention dominates the comparator. ICERs in quadrant $B \& C$ are more difficult to judge as the intervention is either more expensive but results in more effects (B), or the intervention is less expensive but also less effective (C). Hence, one should know how much society is willing to pay for a particular gain in clinical benefits. To help decision-makers in these scenarios, the Dutch Council for Public Health and Healthcare has provided a report with guidance on the ceiling ratios for a QALY for specific disability weights. Based on this report, the ceiling ratio can be roughly estimated to be $€ 20,000-80,000$ per QALY depending on the severity of the disease or disorder. ${ }^{32}$ 


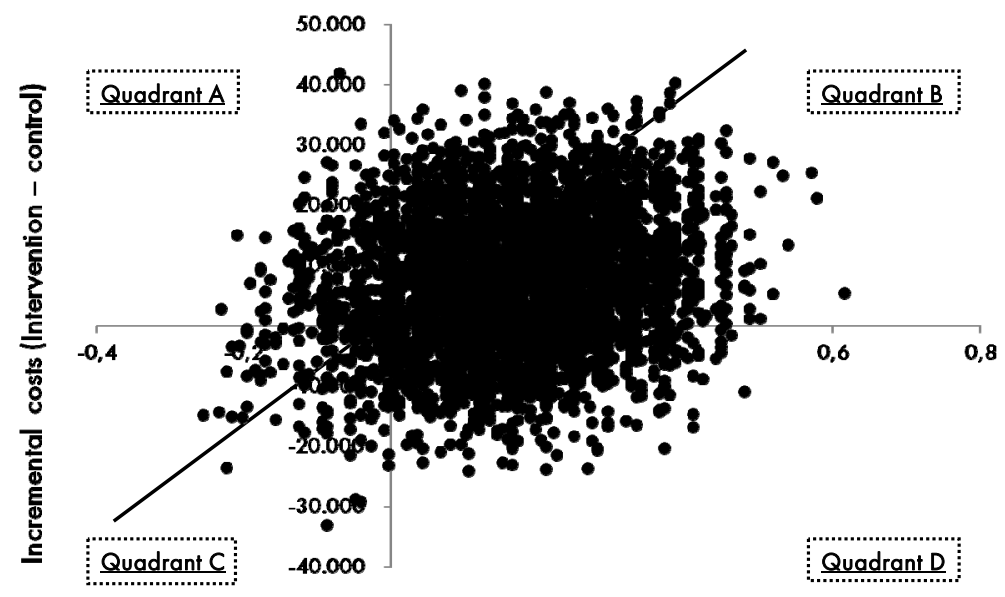

Incremental percentage responders (Intervention - control)

Figure 1.3 Example of a (fictional) cost-effectiveness plane including a fictional threshold.

\subsubsection{Uncertainty encountered in economic evaluations}

One of the most common forms of uncertainty is sampling uncertainty. In every economic evaluation it is possible that, by chance, patients with extremely high or low costs are included in the study which can heavily impact the ICER. To investigate the uncertainty surrounding the ICER, a technique called bootstrapping is often used. When bootstrapping, one creates $N$ times (usually 5000 times) a new sample out of the original sample with replacement. This results in $N$ different samples, each with a different ICER. When plotting these ICERs in a CE-plane, one can visualize the uncertainty around the ICER (see Figure 1.3). Alternatively, one can also calculate the $95 \%$ confidence interval by selecting the $2.5^{\text {th }}$ percentile and $97.5^{\text {th }}$ percentile.

\section{Outline of the present thesis}

The title of this thesis is "Health technology assessment in epilepsy; moving towards patientcentered, efficient care". In order to actually achieve more patient-centered and efficient care, it is important to examine these two domains more carefully. Therefore, the first two parts of this thesis are devoted to: 1) methodological aspects and preference-based studies in the health technology assessment of healthcare interventions, and 2) economic evaluations within epilepsy, as the ultimate goal of most economic evaluations is to assess the efficiency of particular healthcare interventions or treatments. The last part consists of a more elaborate discussion on the results found in this thesis and the implications and 
recommendations for further research. Guided by research questions, the thesis is outlined below.

\subsection{Part I: Methodological aspects and preference-based studies in the health technology assessment of epilepsy}

\section{Research question 1 - What preferences do people have regarding epilepsy diagnostics} and the valuation of clinical manifestations of epilepsy?

Preference studies are a trivial concept within health technology assessment. Preferences can be obtained from specific (epilepsy) patients or from the general public, depending on one's objectives.

Chapter 2 provides insight into patients' preferences regarding epilepsy diagnostics by means of a discrete-choice experiment. In the (primary) diagnosis of epilepsy, it is reasonable to assume that, besides psychometrics, such as sensitivity and specificity, other factors or attributes may play an important role in the willingness of patients to be diagnosed with a certain modality. One could think of attributes like preparation, technique, complication rate, duration, whether or not they have to travel, sound, pain, invasiveness and discomfort. Chapter 2 examines patients' preference for several attributes of diagnostic modalities in the primary diagnosis of epilepsy. A discrete-choice experiment involves a series of hypothetical scenarios (two each time), which consist of several attributes (e.g. travel time, freedom of movement) varying according to level (e.g. 15-60 min travel time; no freedom of movement or freedom of movement is permitted). Each time patients were asked to select the most preferred option.

In chapter 3, preferences are derived from the general public to determine utility values for several epilepsy health states. This is important as, compared to epilepsy-specific outcomes, utilities are easier to compare between diseases and can be used to calculate QALYs. For this study, the time-trade-off methodology was used. Hence, in a situation where only epilepsy-specific outcomes (such as seizure frequency and severity) are available, one could potentially estimate utilities for epilepsy health states.

Research question 2 - How do generic quality of life instruments compare to epilepsyspecific quality of life instruments?

In chapter 4, a generic quality of life instrument is compared with an epilepsy-specific quality of life. The use of generic instruments is important for policy-makers; however, the lack of sensitivity of these instruments may be a matter of concern. If generic instruments are found to be less sensitive to change in patients with epilepsy, interventions may be deemed to be not effective when in fact they are. Given the importance of generic instruments in decision-making, a lack of improvement in quality of life could mean the difference between in- or excluding the (new) treatment in the health insurance package. 
Research question 3 - To what extent is a novel method to elicit preferences used to determine preferences in healthcare?

Chapter 5 describes an examination of the use of best-worst scaling (BWS) in healthcare. As discussed in chapter 2, discrete-choice experiments (DCE) have been used in healthcare since the 1990s for various purposes involving several stakeholders. ${ }^{33}$ The review by Clark et al. ${ }^{33}$ has provided an overview of DCEs in healthcare and may have stimulated the development and the use of DCEs within healthcare. BWS is, however, a relatively novel method to elicit patients' preferences and has been argued to overcome some drawbacks of the DCE. For example, a BWS can include more items than a DCE (especially for BWS case 1), and a BWS is assumed to be easier to complete, as people are, in general, used to discriminating between extremes. The overview of BWS provided in chapter 5 sheds some light on its application in healthcare. This may increase awareness of BWS among researchers in the field of health technology assessment and provide insights into the various purposes for which it can be applied.

\subsection{Part II: Economic evaluations within epilepsy}

Research question 4 - How to appraise economic evaluations and the applicability of an economic evaluation to a particular setting?

When using economic evaluations for decision-making or clinical practice guideline (CPG) development, it is essential to determine their quality and applicability. Many quality and transferability checklists have been developed over the years, and so in chapter 6 we have tried to clarify the situation by providing some guidance as to which checklists could be used when appraising the quality and transferability of economic evaluations.

\section{Research question 5 - What are cost-effective treatments and strategies in epilepsy care?}

A good basis for examining cost-effective treatments in epilepsy, is the identification of all currently available evidence. Chapter 7 reports results of a systematic literature review, focusing on all available treatments for people with epilepsy.

In chapter 8, the long-term (clinical) outcomes of a randomized controlled trial on the cost-effectiveness of the ketogenic diet are presented. In this study, effects and costs of the ketogenic diet were compared to care as usual in children and adolescents with intractable epilepsy.

Chapters $9 \& 10$, respectively, describe the design and cost-effectiveness results of a multi-component intervention for adult patients with epilepsy. Both costs and effects of the intervention and care as usual group were compared. The multi-component intervention was aimed at improving a patient's ability to self-manage his or her disease and at increasing adherence to medication. 


\section{Part III - Discussion and summary}

Chapter 11 is an elaborate discussion on the results found in several chapters of this thesis as well as the implications and recommendations for further research.

Lastly, chapters 12 \& 13 provide a summary of the thesis in both English (chapter 12) and Dutch (chapter 13). 


\section{References}

1. World Health Organization. Epilepsy. 2009.

http://www.who.int/mediacentre/factsheets/fs999/en/index.html.

2. Kotsopoulos I, de Krom M, Kessels F, Lodder J, Troost J, Twellaar $M$ et al. Incidence of epilepsy and predictive factors of epileptic and non-epileptic seizures. Seizure. 2005; 14(3):175-82.

3. Netherlands institute for health services research NIVEL Zorgregistraties eerste lijn. 2015. https://www.nivel.nl/nl/NZR/zorgregistraties-eerstelijn.

4. Jacoby A, Baker GA. Quality-of-life trajectories in epilepsy: a review of the literature. Epilepsy Behav. 2008; 12(4):557-71.

5. Taylor RS, Sander JW, Taylor RJ, Baker GA. Predictors of health-related quality of life and costs in adults with epilepsy: a systematic review. Epilepsia. 2011;52(12):2168-80.

6. Kotsopoulos IA, Evers SM, Ament AJ, de Krom MC. Estimating the costs of epilepsy: an international comparison of epilepsy cost studies. Epilepsia. 2001;42(5):634-40.

7. Kotsopoulos IA, Evers SM, Ament AJ, Kessels FG, de Krom MC, Twellaar M et al. The costs of epilepsy in three different populations of patients with epilepsy. Epilepsy Res. 2003;54(2-3):131-40.

8. de Boer HM, Mula M, Sander JW. The global burden and stigma of epilepsy. Epilepsy Behav. 2008; 12(4):540-6.

9. De Boer HM. "Out of the shadows": a global campaign against epilepsy. Epilepsia. 2002;43 Suppl 6:7-8.

10. Kobau R, Gilliam F, Thurman DJ. Prevalence of self-reported epilepsy or seizure disorder and its associations with self-reported depression and anxiety: results from the 2004 HealthStyles Survey. Epilepsia. 2006;47(1 1):1915-21.

11. Barry JJ, Ettinger AB, Friel P, Gilliam FG, Harden CL, Hermann B et al. Consensus statement: The evaluation and treatment of people with epilepsy and affective disorders. Epilepsy Behav. 2008;13, Supplement $1: S 1-S 29$

12. Hoppe $C$, Elger $C E$, Helmstaedter $C$. Long-term memory impairment in patients with focal epilepsy. Epilepsia. 2007;48 Suppl 9:26-9.

13. Bromfield EB. Epileptiform Discharges. MedScape. 2012.

http://emedicine.medscape.com/article/1138880-overview. Accessed 16-05 2012.

14. Noachtar S, Remi J. The role of EEG in epilepsy: a critical review. Epilepsy Behav. 2009; 15(1):22-33.

15. Poos MJJCS, Smit JM, Groen J, Kommer GJ, Slobbe LCJ. Kosten van ziekten in Nederland 2005. Bilthoven, RIVM. 2008.

16. Elwes R, Marshall J, Beattie A, Newman P. Epilepsy and employment. A community based survey in an area of high unemployment. J Neurol Neurosurg Psychiatry. 1991;54(3):200-3.

17. Hart YM, Shorvon SD. The nature of epilepsy in the general population. I. Characteristics of patients receiving medication for epilepsy. Epilepsy Res. 1995;21(1):43-9.

18. Pato Pato A, Cebrian Perez E, Cimas Hernando I, Lorenzo Gonzalez JR, Rodriguez Constenla I, Gude Sampedro F. Analysis of direct, indirect, and intangible costs of epilepsy. Neurologia. 2011;26(1):32-8.

19. World Health Organization. Health technology assessment. 2016. http://www.who.int/medical_devices/assessment/en/.

20. Bridges J. Stated preference methods in health care evaluation: an emerging methodological paradigm in health economics. Appl Health Econ Health Policy. 2003;2(4):213-24.

21. Bridges JF, Hauber AB, Marshall D, Lloyd A, Prosser LA, Regier DA et al. Conjoint analysis applications in health-a checklist: a report of the ISPOR Good Research Practices for Conjoint Analysis Task Force. Value Health. $2011 ; 14(4): 403-13$.

22. Ryan M, Scott DA, Reeves $C$, Bate A, van Teijlingen ER, Russell EM et al. Eliciting public preferences for healthcare: a systematic review of techniques. Health Technol Assess. 2001;5(5):1-186.

23. Marley AA, Louviere JJ. Some probabilistic models of best, worst, and best-worst choices. J Math Psychol. 2005;49(6):464-80.

24. Finn A, Louviere JJ. Determining the appropriate response to evidence of public concern: the case of food safery. J Public Policy Marketing. 1992:12-25.

25. McIntosh E, Louviere J. Separating weight and scale value: an exploration of best-attribute scaling in health economics. Health Economics Study Group Odense, Denmark. 2002. 
26. Wiinen B, de Kinderen $R$, Colon A, Dirksen $C$, Essers B, Hiligsmann $M$ et al. Eliciting patients' preferences for epilepsy diagnostics: A discrete choice experiment. Epilepsy Behav. 2014;31:102-9.

27. Drummond MF, Sculpher MJ, Claxton K, Stoddart GL, Torrance GW. Methods for the economic evaluation of health care programmes. Oxford university press; 2015.

28. Coons SJ, Rao S, Keininger DL, Hays RD. A comparative review of generic quality-of-life instruments. Pharmacoeconomics. 2000;17(1):13-35.

29. Cramer JA, Perrine K, Devinsky O, Bryant-Comstock L, Meador K, Hermann B. Development and cross-cultural translations of a 31 -item quality of life in epilepsy inventory. Epilepsia. 1998;39(1):81-8.

30. Drost R, Paulus A, Ruwaard D, Evers S. Handleiding intersectorale kosten en baten van (preventieve) interventies: Classificatie, identificatie en kostprijzen. Maastricht: Maastricht University, Department of Health Services Research. 2014.

31. Nederland Z. Richtlijn voor het vitvoeren van economische evaluaties in de gezondheidszorg. Diemen: Zorginstituut Nederland. 2015.

32. Council for Public Health and Health Care. Sensible and sustainable care (in Dutch). Zoetermeer2006.

33. Clark MD, Determann D, Petrou S, Moro D, de Bekker-Grob EW. Discrete choice experiments in health economics: a review of the literature. Pharmacoeconomics. 2014;32(9):883-902. 


\section{Methodological aspects}

and preference based studies in the health technology assessment of epilepsy
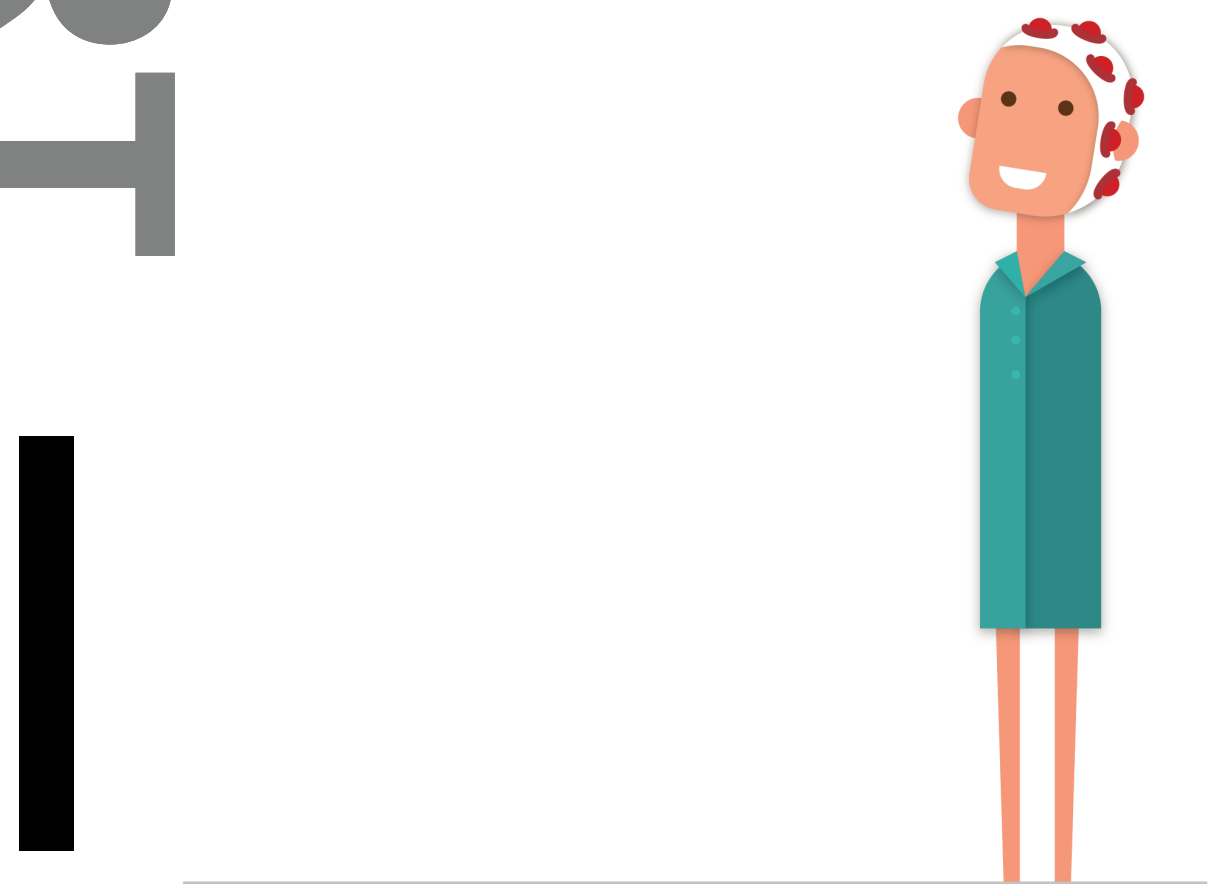



\section{Eliciting patients' preferences}

for epilepsy diagnostics A discrete choice experiment
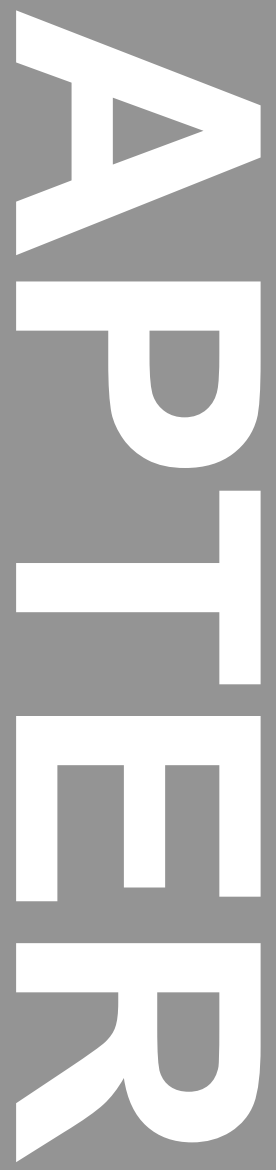

B.F.M. Wiinen R.J.A. de Kinderen A.J. Colon C.D. Dirksen

B.A.B. Essers M. Hiligsmann

F.S.S. Leijten P.P.W. Ossenblok

S.M.A.A. Evers

Epilepsy \& Behavior, 2014;31:102-109 


\section{Abstract}

\section{Background}

Diagnosing epilepsy is a lengthy and burdensome process for patients and their family. Although the need for a more patientcentered approach in clinical practice is widely acknowledged, empirical evidence regarding patient preferences for diagnostic modalities in epilepsy is missing. The objectives of this study are 1) to identify to what extent important attributes of diagnostic procedures in epilepsy affect preferences for a procedure; 2) to determine the relative importance of these attributes; 3) to calculate overall utility scores for routine electroencephalography (EEG) and magnetoencephalography (MEG) recordings.

\section{Methods}

A discrete choice experiment was performed to determine patients' preferences, which involved presentation of pair-wise choice tasks regarding hypothetical scenarios. Scenarios varied along six attributes: "Way of measuring brain activity"; "Duration"; "Freedom of movement"; "Travel time"; "Type of additional examination"; and "Chance of additional examination". Choice tasks were constructed using a statistically efficient design and the questionnaire contained 15 unique unlabeled choice tasks. Mixed multinomial logistic regression was used to estimate patients' preferences.

\section{Results}

A total of 289 questionnaires were included in the analysis. McFadden's pseudo R2 showed a model fit of 0.28 and all attributes were statistically significant. Heterogeneity in preferences was present for all attributes. "Freedom of movement" and "Chance of additional examination" were perceived as the most important attributes. Overall utility scores did not substantially differ between routine EEG and MEG.

\section{Conclusions}

This study suggests that the identified attributes are important in determining patients' preference for epilepsy diagnostics. It can be concluded that MEG is not necessarily more patient-friendly than a routine EEG in primary diagnostics and, regarding additional diagnostics, patients have a strong preference for long-term 24h EEG over EEG after sleep deprivation. Furthermore, barring substantial heterogeneity within the parameters in mind, our study suggests that it is important to take individual preferences into account in medical decision-making. 


\section{Introduction}

Epilepsy is a severe brain disease with a large impact on patients' quality of life, ${ }^{1,2}$ their surrounding and society as a whole. ${ }^{3,4}$ It is one of the most prevalent non-communicable neurologic diseases and estimated to affect almost 70 million people around the world. 5 Internationally, the incidence of epilepsy is estimated at 50 per 100,000 people, with large differences between high-income countries $(45 / 100,000)$ and low- and middleincome countries $(82 / 100,000) .^{5-7}$ Diagnosing epilepsy is a lengthy process that can be a burden for the patient and has a large impact on the health system, while misdiagnoses are quite common. Of patients diagnosed with epilepsy who are presented at epilepsy centers, $20 \%$ to $30 \%$ are found to have been misdiagnosed. ${ }^{8-10}$

According to several European guidelines, ${ }^{11-13}$ it is recommended that an Electroencephalography (EEG) should be performed to support a diagnosis of epilepsy in patients in whom the clinical history suggest that the symptoms are likely to be epileptic in origin. Consequently, EEG is the most used diagnostic procedure for epilepsy. ${ }^{14}$ However, its sensitivity and specificity depends on several factors such as age and recording procedures (e.g. activation procedures such as provoked hyperventilation or photic stimulation). ${ }^{14}$ A routine EEG recording in a patient with epilepsy will show no epileptiform activity in about $50 \%$ of cases. ${ }^{15}$ Hence, additional EEG examinations are needed to increase diagnostic yield by increasing epileptiform activity without inducing seizures. To this extent, EEG after sleep deprivation (EEGsd) and long-term 24h-EEG are generally recommended. ${ }^{11-13,15}$ The latter is often used in children or mentally ill patients, since an EEGsd is considered to be too burdensome for these patients.

Nonetheless, both 24h-EEG and EEGsd, have considerable disadvantages. Sleep deprivation is shown to elicit seizures, ${ }^{15-18}$ which is supported by the fact that it is still one of the three most important seizure inducing factors mentioned by patients in questionnaires. $^{19-21}$ The $24 \mathrm{~h}-\mathrm{EEG}$ impairs patients in their daily routine; wires attached to the portable EEG-recorder refrain them from physical exercises and limit their freedom of movement.

An alternative to these diagnostic procedures is magnetoencephalography (MEG), which seems to be a more patient friendly way to detect epileptiform discharges, ${ }^{22}$ potentially with great diagnostic value in the early stage of the diagnostic process. ${ }^{22-24}$ MEG is a non-invasive technique that enables recording of epileptiform discharges over the whole head, with minimal preparations. Nevertheless, MEG also has some considerable disadvantages; most importantly a MEG recording requires that patient's move as little as possible, which could be very limiting for some patients.

It is important to include patients' preferences in evaluation of health technologies as patients differ from each other in their biologic, social or cultural characteristics as well as in their preferences. Although the need for a more patient-centered approach in clinical practice and evaluation studies is widely acknowledged ${ }^{25-27}$ and patients preferences have been shown to significantly impact their willingness to use healthcare services, ${ }^{28-30}$ empirical evidence is missing regarding patients' preferences for routine EEG, EEGsd, 
24h-EEG, and MEG as diagnostic tools in epilepsy. Using a discrete choice experiment (DCE), the present study attempted to determine patients' preferences for different elements of the diagnostic trajectory of epilepsy. The DCE involved presentation of a series of pairwise choice tasks regarding hypothetical scenarios. ${ }^{31,32}$ DCEs have increasingly been used in health care as an approach to elicit patients' preferences for medication attributes and have been applied in similar cases (e.g. patients' preferences regarding several screening tests). ${ }^{33-37}$

Therefore, the objectives of this study are 1) to identify to what extent important attributes of diagnostic procedures in epilepsy affect patients' preferences for a procedure; 2) to determine the relative importance of these attributes; and 3) to calculate overall utility scores for routine EEG and MEG recordings.

\section{Methods}

\subsection{Discrete choice experiment}

A DCE is an attribute-based stated preference valuation technique. ${ }^{32}$ In DCEs, individuals are presented with a series of hypothetical choice tasks and are asked to express a preference. The technique is based on the premises that healthcare interventions can be described by a number of attributes and that preferences for these interventions are influenced by the variations (i.e. levels) within these attributes (see Table 2.1). ${ }^{32}$

Using these attributes and levels, one can construct a "scenario" by choosing a level for each attribute. Subsequently, respondents are asked to indicate, by pairwise comparison of alternative scenarios, which would be their preferred option (see Figure 2.1). In this way, respondents are encouraged to make a trade-off between attributes and their corresponding levels. Afterwards, the relative importance of each attribute (and corresponding levels) can be identified using a regression model.

In this study, an unlabeled DCE is used (i.e. the alternatives are named method $A$ and method $B$, instead of e.g. EEG and MEG), mainly because the labeled alternatives themselves convey information to respondents. Thus, in a labeled design, respondents may use labeled alternatives to deduce information which they perceive as missing, particularly when patients are familiar with some of the alternatives. ${ }^{38}$ Hence, an unlabeled DCE might encourage respondents to choose an alternative by only trading-off attribute levels. ${ }^{38}$

DCE methodology is well described in the literature. ${ }^{31,32}$ This study was conducted according to the stepped approach proposed by Ryan and Farrar. ${ }^{39}$ 
Table 2.1 Attributes and levels for epilepsy diagnostics

\begin{tabular}{ll}
\hline Attributes & Levels \\
\hline Way of measuring brain activity & Sensor net (Base case ${ }^{\star}$ ) \\
& Helmet \\
& Electro cap \\
Duration of recording * & 30 minutes \\
& 60 minutes \\
& 90 minutes \\
Freedom of movement & Minor movements possible (Base case ${ }^{*}$ ) \\
& No movements possible \\
Travel time & 30 minutes \\
& 60 minutes \\
& 90 minutes \\
Type of additional examination & $24 \mathrm{~h}$-EEG (Base level ${ }^{*}$ ) \\
& EEG after sleep deprivation \\
Chance of additional examination * & 20 out of 100 patients \\
& 35 out of 100 patients \\
& 50 out of 100 patients \\
\hline
\end{tabular}

* Level of the attribute which was used as the reference level; ** Estimated as continuous variable within the MMNL model

\begin{tabular}{|l|c|c|}
\hline Characteristics & Method A & Method B \\
\hline Way of measuring brain activity & By use of a helmet & By use of an electro cap \\
\hline Duration of recording & 30 minutes & 90 minutes \\
\hline Freedom of movement & No movements possible & Minor movements possible \\
\hline Travel time & 30 minutes & 90 minutes \\
\hline Type of additional examination & EEG after sleep deprivation & 24 -hour EEG \\
\hline Chance of additional examination & $50 \%$ (50 out of 100 patients) & $20 \%$ (20 out of 100 patients) \\
\hline Which option do you prefer? & $\square$ & $\square$ \\
\hline
\end{tabular}

Figure 2.1 Example of unlabeled choice set as presented to patients

\subsection{Identifying attributes and levels}

Attributes were identified based on literature review, ${ }^{11,12,14,15,22}$ expert and patient interviews ( $n=7$, three neurologists; two patients and two laboratory technicians) and observations during clinical practice. Expert and patient interviews were semi-structured to give participants the opportunity to explain their thoughts rather than forcing them to choose from fixed responses. In our case, due to the impact on the random component variability ${ }^{40}$ and to minimize cognitive burden, ${ }^{41}$ the number of attributes was limited to six. Other attributes were considered to be less relevant or were left out due to commonality between attributes. The final attributes, based on a consensus meeting with clinicians and experts, were: 1) way of measuring brain activity, 2) duration of the recording (DURATION), 3) freedom of movement (FOM), 4) travel time (TT), 5) type of additional examination (TAE), and 6) chance of additional examination (CAE) (see Table 2.1). Levels for the first attribute were chosen to represent a routine EEG recording (electro cap); MEG recording (helmet); and routine EEG recording with a HydroCel Geodesic Sensor Net. ${ }^{42}$ 
The addition of the sensor net, which is said to not require any scalp preparation or abrasion, was merely to see what the impact of future improvements in EEG recording might offer to patients.

Levels for the attribute "Chance of additional examination" were based on previous studies regarding the sensitivity of MEG and EEG. ${ }^{14,22,23,43,44}$ Other levels were determined based on expert opinion (i.e. travel time; and type of additional examination) and clinical characteristics of each recording (i.e. duration of recording; and freedom of movement). Travel time was taken into account as up to now the availability of MEG in the Netherlands is limited.

\subsection{Designing choice sets and questionnaire}

The choice sets are constructed using Ngene (Version 1.1.1, http://www.choicemetrics.com/). Four attributes with three levels, and two attributes with two levels, result in $3^{4} * 2^{2}=324$ hypothetical scenarios. Hence, for practical reasons, a fractional factorial design (subset of hypothetical scenarios) was used.

The fractional factorial design was constructed using Bayesian principles, a so-called Bayesian optimal or statistically efficient design. Using an efficient design it is possible to maximize the precision of estimated parameters for a given number of choice tasks (by maximizing D-efficiency). ${ }^{45,46}$ The statistically efficient design approach assumes a prior distribution of likely parameter values (e.g. the beta coefficients in the regression analysis) for some or all parameters. ${ }^{47}$ For example, it is likely to assume that patients prefer a shorter duration of recording and travel time. Therefore, these attributes were assumed to have a negative sign (i.e. longer duration decreases patients' preferences).

Within each choice task, respondents were asked to choose between two unlabeled scenarios (see Figure 2.1). We did not include an opt-out option representing the alternative 'no diagnostic testing'. This way, respondents were forced to make trade-offs between attributes and it ensured that respondents could not avoid difficult decisions. ${ }^{48,49}$ Moreover, little information is captured in terms of attribute level trade-offs when such an option is selected.

After a short introduction to the study, respondents were given a detailed description about the DCE task. Next, attributes and levels were explained and an example of a choice set was shown. Subsequently, the choice tasks were presented and, lastly, respondent characteristics were collected.

The final version of the questionnaire contained 15 unique choice tasks. To evaluate the test-retest reliability of the questionnaire, one choice task was repeated at the end of the questionnaire. This makes a total of 16 choice tasks per respondents, which is considered good practice and cognitively acceptable. ${ }^{50,51}$ Afterwards, respondents were asked to evaluate the difficulty of the tasks on a seven-point scale ranging from "very easy" to "very difficult". The questionnaire was pilot tested among experts to ensure that the levels of each choice were relevant and comprehensible. The full questionnaire in Dutch is available from the authors on request. 


\subsection{Data collection and participants}

The study was conducted in the outpatient clinic of Kempenhaeghe, expertise centre in the field of epilepsy, and at the University Medical Centre Utrecht (UMCU). Both involved parties have a leading role in the application of MEG in clinical epilepsy research.

Data was collected between February and April 2013. The DCE questionnaire was sent by mail to 1009 patients, completed by the patients at home and returned in a postage-paid envelope. All patients had to be at least 18 years of age, diagnosed as having epilepsy, visited the outpatient clinic of Kempenhaeghe in the past year and homedwelling. Patients living in specialized healthcare institutions (i.e. for mentally handicapped patients) were excluded from the study. Due to the limited application of MEG in primary diagnostics of epilepsy, we deliberately contacted patients from UMCU, experienced with MEG, to ensure that these patients were represented in the sample.

Only patients who agreed to be contacted for participation in (clinical) studies were approached. An information letter attached to the questionnaire was sent to these patients, which explained that by returning the questionnaire respondents consented to participate in the study. Approval for this consent procedure and study was obtained from the ethics committee of Kempenhaeghe and UMCU.

\subsection{Statistics and data analysis}

Discrete choice data were analyzed using Nlogit version 5 (Econometric Software, Inc). A mixed multinomial logit model ( $M M N L)$ was used to determine the effect of the attribute levels on patients' preferences. Respondents who completed less than five choice sets were excluded from the analysis.

MMNL was chosen to allow for possible preference heterogeneity across respondents and to account for the panel nature of the data. ${ }^{38,52}$ A MMNL model is constructed regarding the assumption that parameters are randomly distributed in the population. Heterogeneity within these parameters can be assessed by estimating the standard deviation of the parameters. ${ }^{46}$ Therefore, MMNL is able to relax some or all of the assumptions of the standard multinomial logit model, most importantly homogeneity of patients' preferences. $^{52}$

The six attributes in the DCE were modelled as determinants of whether respondents chose "Method A" or "Method B", hence the difference in utility between both scenarios. Therefore, the regression function to be estimated had the following form:

$$
\begin{aligned}
& V_{\mathrm{ii}}=\left(\beta_{1}+\eta_{1 \mathrm{i}}\right) * \text { HELMET }+\left(\beta_{2}+\eta_{2 \mathrm{i}}\right) \text { * ELECTROCAP } \\
& +\left(\beta_{3}+\eta_{3 \mathrm{i}}\right){ }^{*} \text { DURATION }+\left(\beta_{4}+\eta_{4 \mathrm{i}}\right) * \text { FOM }+\left(\beta_{5}+\eta_{5 i}\right) * \mathrm{TT}+\left(\beta_{6}+\eta_{6 \mathrm{i}}\right) \\
& \text { TAE }+\left(\beta_{7}+\eta_{7 \mathrm{i}}\right) * \text { CAE }+\varepsilon_{\mathrm{ij}}
\end{aligned}
$$

In which the value of the regression coefficients indicate the relative importance of the attribute; $V$ represents the observable relative utility; and $\eta i$ represents the standard deviation of the random parameter. Lastly, $\eta_{i j}+\varepsilon i i$ captures the individual-specific 
unexplained variance around the mean. ${ }^{46}$ No constant term was included in the final model as the constant term represents the mean of the unobserved effects for each of the alternatives, after accounting for the role each attribute plays in the decision making process. ${ }^{52}$ Hence, in an unlabeled design, a significant constant term would be an indication of left-to right bias (i.e. tendency to consistently choose either the first or the second alternative in the choice task). ${ }^{48}$ To describe categorical variables, dummy coding was used for ease of interpretation, base-case levels can be found in Table 2.1.

We tested interactions between attributes and between attributes and respondent characteristics. All parameters included in the MMNL model were treated as random parameters and estimated using 2000 Halton draws. Parameters were tested for significance at $\alpha=0.05$ and assumed to be normally distributed. Next, model fit was assessed using log-likelihood and McFadden's pseudo- $R^{2}$. To test for left-to right bias the constant term was included in the initial analyses. To account for scale differences and ease of interpretation, relative importance weights were calculated using the method described by Malhotra and Birks. ${ }^{53}$ For continuous variables we based this calculation on the total range of the attribute.

Reliability analyses were conducted to explore the impact of respondents who failed the test-retest and to explore the impact of differences in perceived difficulty. Additional analyses were performed on age (two groups; split by mean value), educational level (two groups, split by mean value), and gender, by means of creating interaction terms within the final model. These analyses were done to assess the potential impact of covariates and to explain some heterogeneity within the data.

Results of the MMNL model were translated to clinical practice. As mentioned above, levels for each attribute were chosen based on expert opinion and clinical characteristics of each recording. Using this information, one can (hypothetically) compare routine EEG and MEG by selecting levels which closely resemble clinical features of each recording and calculate overall utility scores using the utility function derived by the MMNL model. The lower the overall utility score, the less preferred that scenario is.

Data file and additional analyses can be retrieved by contacting the corresponding author.

\section{Results}

\subsection{Respondent characteristics}

A total of 304 respondents (30.1\%) completed the DCE questionnaire. Excluding respondents who completed five choice tasks or less, a total of 289 questionnaires were included in the analysis. Mean age of the respondents was 45.5 years. Nearly all respondents reported to have experience with epilepsy diagnostic using brain imaging techniques; only $5.9 \%$ reported not to have experience with any of the techniques. Other patient characteristics are presented in Table 2.2. No significant differences were found between responders and non-responders for all respondent characteristics. 
Table 2.2 Respondent characteristics

\begin{tabular}{lrr}
\hline Characteristic & & Total cohort (N = 289) \\
\hline Gender & Male & $130(45 \%)$ \\
& Female & $158(54.7 \%)$ \\
Agely) & Missing & $1(0.3 \%)$ \\
& Mean & 45.5 years \\
& $20-34$ & $80(27.7 \%)$ \\
& $35-49$ & $91(31.5 \%)$ \\
& $50-64$ & $82(28.4 \%)$ \\
& $65-85$ & $33(11.4 \%)$ \\
& Missing & $3(1.0 \%)$ \\
& Primary & $83(28.7 \%)$ \\
Intervention experience * & Secondary education & $123(42.6 \%)$ \\
& Secondary vocational education & $55(19.0 \%)$ \\
& Higher education & $22(7.6 \%)$ \\
& Missing & $6(2.1 \%)$ \\
& Electro cap & $219(75.8 \%)$ \\
& Helmet & $63(21.8 \%)$ \\
& EEGsd & $152(52.6 \%)$ \\
& $24 h-E E G$ & $164(56.7 \%)$ \\
& No experience & $17(5.9 \%)$ \\
& Missing & $2(0.7 \%)$ \\
& Relatively easy (range: $1-3)$ & $114(39.4 \%)$ \\
& Neutral (range: 4$)$ & $61(21.1 \%)$ \\
& Relatively difficult (range: $5-7)$ & $102(35.3 \%)$ \\
& Missing & $12(4.2 \%)$ \\
\hline
\end{tabular}

* The "sensor net" is not in this table because it is not used in routine practice; thus no respondent could have experience with it.

\subsection{DCE choice task}

McFadden's pseudo $R^{2}$ shows a model fit of 0.28 . All attributes are statistically significant, which provides evidence of the theoretical validity of the model and indicates that the attributes are considered as being important in determining patients' preferences. $^{28}$ The regression coefficient for each attribute level represents the mean part-worth utility of that attribute level in the respondent sample. A negative $\beta$-coefficient indicates that as the level of the attribute increases, utility is decreasing. Results of the MMNL model are presented in Table 2.3.

The estimated coefficients all have the anticipated negative sign, apart from "Way of measuring brain activity" and "Type of additional examination", of which no specific effect was anticipated. 
Table 2.3 Results from mixed multinomial logit model illustrating influence of attributes on utility

\begin{tabular}{lll}
\hline Attributes & Coefficient $(95 \% \mathrm{Cl})$ & Standard deviation $(95 \% \mathrm{Cl})$ \\
\hline Way of measuring brain activity & & \\
Helmet & $-0.220(-0.445,-0.005)^{* *}$ & $1.243(0.993,1.492)^{* * *}$ \\
Electro cap & $-0.017(-0.203,0.169)$ & $0.896(0.651,1.142)^{* * *}$ \\
Duration (per minute) & $-0.018(-0.022,-0.015)^{* * *}$ & $0.016(0.013,0.020)^{* * *}$ \\
Freedom of movement & $-1.840(-2.117,-1.563)^{* * *}$ & $1.783(1.509,2.056)^{* * *}$ \\
Travel time (per minute) & $-0.015(-0.019,-0.012)^{* * *}$ & $0.020(0.016,0.024)^{* * *}$ \\
Type of additional examination & $-0.939(-1.206,-0.671)^{* * *}$ & $2.001(1.711,2.291)^{* * *}$ \\
Chance of additional examination (\%) & $-0.057(-0.070,-0.045)^{* * *}$ & $0.079(0.067,0.092)^{* * *}$ \\
Log likelihood & -2164.86 & \\
Pseudo R-squared & 0.28 & \\
Number of observations & 4335 & \\
Number of individuals & 289 & \\
\hline
\end{tabular}

$* * *, * *, *$ Significance at $1 \%, 5 \%, 10 \%$ level. Table represents $\beta$-coefficients from MMNL model. The regression coefficients represent the mean part-worth utility of that attribute in the respondent sample. $\mathrm{Cl}$ : Confidence interval.

To determine which attribute has the largest impact on patients' preferences, it is convenient to look at the relative importance weights as they represent the importance of each attribute in percentages (see Table 2.4). As shown, "Freedom of movement" has the largest impact on patients' preference (27\%). Respondents had a significant preference for scenarios in which minor movements were possible. The attribute with the second largest impact on patients' preference was "Chance of additional examination" (26\%), indicating respondents preferred a lower chance of additional examination. Furthermore, respondents had a significant preference for shorter recording (16\%), shorter travel times $(13 \%)$, and significantly preferred $24 \mathrm{~h}-\mathrm{EEG}$ over EEGsd (14\%). As for the way of measuring brain activity $(4 \%)$, respondents significantly preferred the sensor net over the helmet, but had no significant preference between the sensor net and the electro cap.

Furthermore, standard deviations of the random parameters were significant for all attributes, which is an indication of preference heterogeneity within the sample. Hence, this shows the presence of variations in the importance of attributes between respondents. ${ }^{46,52}$

Table 2.4 Relative importance of attributes (in percentage) for the total sample, males and females

\begin{tabular}{lccc}
\hline Attribute & Total sample (N=289) & $\begin{array}{c}\text { Males } \\
(\mathrm{N}=130)\end{array}$ & $\begin{array}{c}\text { Females } \\
(\mathrm{N}=158)\end{array}$ \\
\hline Way of measuring brain activity & $4 \%$ & $9 \%$ & $2 \%$ \\
Duration & $16 \%$ & $10 \%$ & $17 \%$ \\
Freedom of movement & $27 \%$ & $23 \%$ & $31 \%$ \\
Travel time & $13 \%$ & $21 \%$ & $17 \%$ \\
Type of additional examination & $14 \%$ & $11 \%$ & $16 \%$ \\
Chance of additional examination & $26 \%$ & $26 \%$ & $17 \%$ \\
\hline
\end{tabular}




\subsection{Differences in preferences between subgroups}

Different subgroup analyses, based on interaction terms within the final model, were examined. Models with interactions between attributes and covariates (educational level, age, experience with MEG/EEG(sd/24h), difficulty) were tested, but all covariates turned out to be non-significant. However, a significant difference was found between males and females. Results of the model for male and female respondents are presented in Table 2.5. Female respondents had a significantly stronger preference for recordings in which minor movements were possible, as can also be seen when looking at the difference in relative importance weights (see Table 2.4). Stratified analysis based on gender had large implications on the relative importance weights. In males, "Chance of additional examination" turns out to be the most important attribute (26\%); whereas in females "Freedom of movement" is still the most important attribute (31\%). Preference for other attributes did not significantly differ between males and females.

\subsection{Reliability and difficulty}

A total of 248 respondents (85.8\%) successfully completed the test-retest exercise. Analyses excluding respondents who failed the test-retest showed a significant difference between the relative importance of the "Electro cap". Respondents who successfully completed the test-retest exercise significantly preferred a sensor net over an electro cap, whereas respondents who failed the test-retest had no significant preference between the two modalities (see Table 2.6). Excluding respondents who failed the test-retest exercise improved McFadden's pseudo- $R^{2}$ to 0.30 .

Difficulty of the task was estimated on average at 3.78 on a scale from 1 to 7 . The task was perceived relatively easy (score 1 to 3 ) by 114 respondents (39.4\%) while 102 respondents (35.3\%) perceived the task as relatively difficult (score 5 to 7 ). No significant differences were found between respondents who perceived the task as relatively difficult and respondents who perceived the task as relatively easy. Excluding respondents who perceived the task as extremely difficult (score 7), only marginally improved McFadden's pseudo $R^{2}$ (from 0.275 to 0.281 ). 
Table 2.5 Differences between male and female respondents in their preferences for epilepsy diagnostics

\begin{tabular}{|c|c|c|c|}
\hline Attributes & Males $(95 \% \mathrm{CI})$ & Females $(95 \% \mathrm{Cl})$ & P Value $^{\dagger}$ \\
\hline \multicolumn{4}{|l|}{ Way of measuring brain activity } \\
\hline Helmet & $\begin{array}{l}-0.36(-0.66,-0.06)^{* *} \\
S D: 1.16^{* * *}\end{array}$ & $\begin{array}{l}-0.09(-0.38,0.20) \\
S D: 1.30^{* * *}\end{array}$ & 0.25 \\
\hline Electro cap & $\begin{array}{l}-0.16(-0.43,0.11) \\
S D: 0.84^{* * *}\end{array}$ & $\begin{array}{l}0.05(-0.19,0.29) \\
\text { SD: } 0.74^{* * *}\end{array}$ & 0.64 \\
\hline Duration (per minute) & $\begin{array}{l}-0.01(-0.02,-0.01)^{* * *} \\
S D: 0.02^{* * *}\end{array}$ & $\begin{array}{l}-0.02(-0.03,-0.02)^{* * *} \\
\text { SD: } 0.01^{* * *}\end{array}$ & 0.28 \\
\hline Freedom of movement & $\begin{array}{l}-1.36(-1.74,-0.99)^{* * *} \\
\text { SD: } 1.65^{* * *}\end{array}$ & $\begin{array}{l}-2.14(-2.55,-1.73)^{* * *} \\
S D: 1.97^{* * *}\end{array}$ & $0.01 * *$ \\
\hline Travel time (per minute) & $\begin{array}{l}-0.02(-0.02,-0.01)^{* * *} \\
\text { SD: } 0.02 * * *\end{array}$ & $\begin{array}{l}-0.02(-0.02,-0.01)^{* * *} \\
\text { SD: } 0.02 * * *\end{array}$ & 0.71 \\
\hline Type of additional examination & $\begin{array}{l}-0.64(-0.10,-0.27)^{* * *} \\
S D: 1.96^{* * *}\end{array}$ & $\begin{array}{l}-1.12(-1.47,-0.76)^{* * *} \\
S D: 1.91^{* * *}\end{array}$ & 0.25 \\
\hline \multirow[t]{2}{*}{$\begin{array}{l}\text { Chance of additional examination } \\
\text { (per \%) }\end{array}$} & $-0.05(-0.07,-0.04)^{* * *}$ & $-0.04(-0.06,-0.03)^{* * *}$ & $0.08^{*}$ \\
\hline & SD: $0.09 * * *$ & SD: $0.07^{* * *}$ & \\
\hline Log likelihood & -1029.49 & -1134.19 & \\
\hline Pseudo R-squared & 0.24 & 0.31 & \\
\hline Number of observations & 1965 & 2385 & \\
\hline Number of individuals & 130 & 158 & \\
\hline
\end{tabular}

$* * *, * *,{ }^{*}$ Significance at $1 \%, 5 \%, 10 \%$ level; SD: standard deviation; ${ }^{\dagger} \mathrm{p}$ Value was estimated in a joint model with interaction terms.

Table 2.6 Differences between respondents who passed the test-retest exercise and respondents who failed the test-retest exercise in their preferences for epilepsy diagnostics

\begin{tabular}{|c|c|c|c|}
\hline Attributes & $\begin{array}{l}\text { Passed test-retest exercise } \\
(95 \% \mathrm{Cl})\end{array}$ & $\begin{array}{l}\text { Failed test-retest exercise } \\
(95 \% \mathrm{Cl})\end{array}$ & P Value \\
\hline \multicolumn{4}{|l|}{ Way of measuring brain activity } \\
\hline Helmet & $\begin{array}{l}-0.22(-0.47,0.02)^{*} \\
S D: 1.29^{* * *}\end{array}$ & $\begin{array}{l}-0.25(-0.67,0.18) \\
\text { SD: } 0.93^{* * *}\end{array}$ & 0.46 \\
\hline Electro cap & $\begin{array}{l}-0.16(-0.38,0.04) \\
S D: 0.91^{* * *}\end{array}$ & $\begin{array}{l}0.36(0.04,0.67)^{* *} \\
\text { SD: } 0.04\end{array}$ & $0.04^{* *}$ \\
\hline Duration (per minute) & $\begin{array}{l}-0.02(-0.02,-0.02)^{* * *} \\
\text { SD: } 0.02 * * *\end{array}$ & $\begin{array}{l}-0.01(-0.01,0.00)^{* * *} \\
\mathrm{SD}: 0.01^{* * *}\end{array}$ & 0.67 \\
\hline Freedom of movement & $\begin{array}{l}-2.01(-2.35,-1.67)^{* * *} \\
\text { SD: } 1.92^{* * *}\end{array}$ & $\begin{array}{l}-1.07(-1.44,-0.7)^{* * *} \\
\text { SD: } 0.71 * * *\end{array}$ & 0.85 \\
\hline Travel time (per minute) & $\begin{array}{l}-0.02(-0.02,-0.01)^{* * *} \\
\text { SD: } 0.02 * * *\end{array}$ & $\begin{array}{l}-0.01(-0.01,0.0)^{* * *} \\
\mathrm{SD}: 0.01^{* * *}\end{array}$ & 0.89 \\
\hline Type of additional examination & $\begin{array}{l}-0.87(-1.19,-0.55)^{* * *} \\
\text { SD: } 2.40^{* * *}\end{array}$ & $\begin{array}{l}-0.18(-0.45,0.10) \\
S D: 0.50 * * *\end{array}$ & 0.12 \\
\hline \multirow[t]{2}{*}{$\begin{array}{l}\text { Chance of additional } \\
\text { (per } \%)\end{array}$} & $-0.05(-0.06,-0.04)^{* * *}$ & $-0.03(-0.05,-0.01)^{* * *}$ & 0.12 \\
\hline & SD: $0.08 * * *$ & SD: $0.05^{* * *}$ & \\
\hline Log likelihood & -1824.52 & -335.42 & \\
\hline Pseudo R-squared & 0.30 & 0.16 & \\
\hline Number of observations & 3795 & 540 & \\
\hline Number of individuals & 248 & 36 & \\
\hline
\end{tabular}

$* * *, * *,{ }^{*}$ Significance at $1 \%, 5 \%, 10 \%$ level; SD: standard deviation; $\dagger p$ Value was estimated in a joint model with interaction terms. 


\subsection{Translation to clinical characteristics of diagnostic modalities}

Overall utility scores for routine EEG and MEG were -5.97 and -6.12 (see Figure 2.2). Hence, in general, albeit the difference is marginal, routine EEG is preferred over MEG. However, when stratifying for gender, it can be seen that males prefer MEG over routine EEG whereas females prefer routine EEG over MEG. As this study mainly focuses on discomforts of each modality, overall utility scores are negative. However, this does not imply that patients would prefer to not be diagnosed with EEG or MEG at all as an opt-out option was not included into the choice tasks.

\section{Discussion}

To the best of our knowledge, this is the first study to investigate patients' preferences for brain imaging techniques in the primary diagnosis of epilepsy. This study was designed to identify to what extent important attributes of diagnostic procedures in epilepsy affected preferences for a procedure and to determine the relative importance of these attributes.

The overall fit of the model, as assessed with McFadden's pseudo $R^{2}$, was 0.28 and, when excluding respondents who failed the test-retest exercise, it increased to 0.30 . Both represent a decent model fit for a discrete choice model, as a pseudo $R^{2}$ of 0.30 represents a $R^{2}$ of approximately 0.6 for the equivalent $R^{2}$ of a linear regression model. ${ }^{52}$ Our results indicate that respondents would prefer a test with a short duration, a short travel time, in which minor movements are possible, the electric activity of the brain should be recorded by either a sensor net or an electro cap, a low chance of additional examination and, when additional examinations are necessary, it should preferably be $24 \mathrm{~h}-\mathrm{EEG}$.

When looking at the relative importance weights, two attributes stand out: "Chance of additional examinations" and "Freedom of movement". The attribute "Chance of additional examination", was expected to have a large influence as it relates to the sensitivity of a recording, which is often shown to be of great impact in preference studies. ${ }^{29,54,55}$ Regarding "Freedom of movement", we anticipated the fact that patients would prefer recordings in which minor movements are possible. However, on forehand, it was not entirely evident to assume that it would be the most influential factor in determining patients' preferences. Given these importance weights, one could argue that, albeit the presumed higher sensitivity and shorter duration of MEG, it is not straightforward to assume that, in general, patients would prefer MEG over routine EEG as shown in Figure 2.2. The comparison between both types of additional examination, EEGsd and 24h-EEG, is more clear-cut. For additional diagnostics, this study suggest that patients have a strong preference for 24h-EEG over EEGsd, which could be explained by the fact that sleep deprivation may induce seizures and therefore, patients might try to avoid it. In current practice, $24 \mathrm{~h}-\mathrm{EEG}$ is often used in mentally ill patients or children. However, this study suggests that $24 \mathrm{~h}-\mathrm{EEG}$ could be a more patient-friendly alternative for other patients as well. 


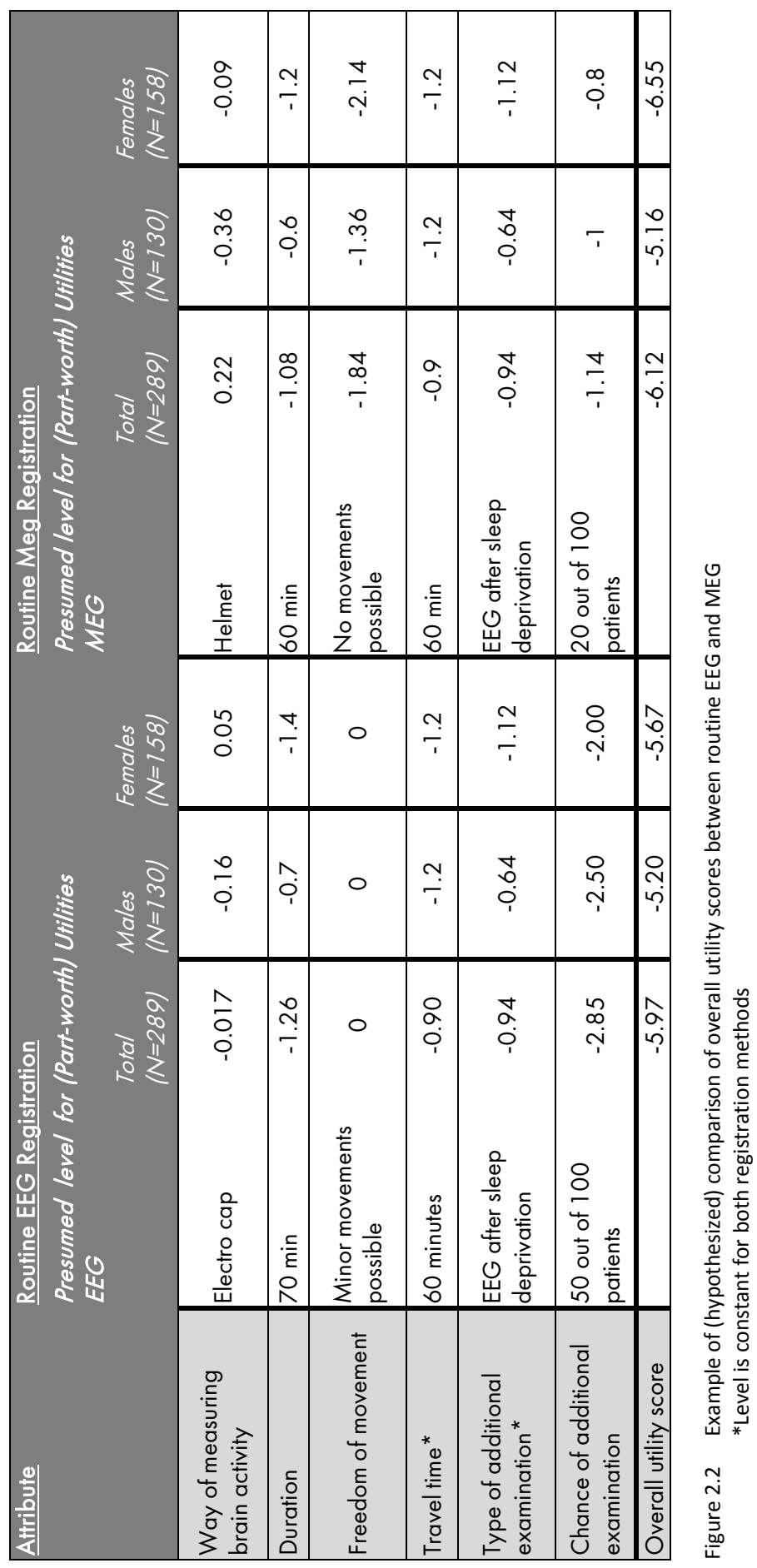


All parameters contained substantial heterogeneity, which indicates differences in the relative importance of the attributes between respondents. A part of the heterogeneity could be explained by the interaction between males and females regarding the attribute "Freedom of movement" and by the interaction regarding the level "Electro cap" between respondents who successfully completed the test-retest exercise and who failed the testretest exercise. These interactions highlight the fact that preferences could be substantially different between subgroups and even between individuals within a subgroup. For example, differences between males and females regarding "Freedom of movement" could have an impact on their preferred diagnostic modality. Other factors explaining the heterogeneity in preferences could not be identified from the available data.

This study has several limitations. First, as Kempenhaeghe and UMCU are expertise centers in the field of epilepsy, often the most complex or severe patients are being referred to these institutes. This is also supported by the fact that a relatively large percentage of the respondents $(56.7 \%$ ) had experience with $24 \mathrm{~h}-\mathrm{EEG}$, which is regularly only used in a specific subset of the patients. Therefore, selection of the sample could potentially be a problem and our findings may only be limited generalizable to the overall epilepsy-care within the Netherlands.

Second, we encountered a response rate of $30.1 \%$ which is moderately low. Response rates to postal questionnaires are approximately $62 \%$, ${ }^{56}$ however regarding DCEs large variations are reported ranging from $10 \%$ to $98 \% .^{57,58}$ Some respondents reported difficulties when filling in the questionnaire (i.e. cognitive burden or too much attributes to take into account) which could be an explanation for the low response rate. The DCE method can be cognitively demanding which could lead to biased results by reducing respondents' ability to make a well-considered choice between different scenarios, especially because epilepsy is often associated with cognitive impairments. ${ }^{41,59}$ However, stratified analyses between respondents who perceived the task as relatively difficult and respondents who perceived the task as relatively easy, did not show any significant differences. Furthermore, no differences were found between patients with different educational levels.

Lastly, in our sample a reasonable amount of respondents (21.8\%) reported experience with MEG recording, which is relatively high as MEG is currently only used as pre-surgical evaluation instrument or in scientific research. In addition, a reasonable amount of respondents (24.2\%) reported to have not been diagnosed with routine EEG. Hence, given that in 2012, according to the Dutch Taskforce for Epilepsy Surgery, approximately 180 patients were referred for pre-surgical evaluation and the fact that a routine EEG is recommended as a first step of the diagnostic process in all European guidelines, $^{11-13}$ recall bias is likely to be present within our sample. However, we have to rely on data as reported by respondents.

In conclusion, our study suggests that the way brain activity is measured, duration of the recording, freedom of movement, travel time, type of additional examination, and chance of additional examination are all important attributes in determining patients' preference for epilepsy diagnostics. The attributes with the largest impact on patients' preferences are 
freedom of movement and type of additional examination. Translated to clinical practice, this means that MEG is not necessarily more patient-friendly than a routine EEG, although an external validation study, comparing revealed preferences in patients experiencing both routine EEG and MEG, would be required to confirm this finding. Furthermore, regarding additional diagnostics, it can be concluded that patients have a strong preference for 24h-EEG over EEGsd, which could help physicians in medical decisionmaking. Additionally, barring the substantial heterogeneity within the parameters in mind, it is important to take individual preferences into account in medical decision-making to improve epilepsy diagnostics.

We believe that this study can be a valuable contribution to patient-centered care within epilepsy diagnostics. Understanding and incorporating patients' preferences in the diagnostic trajectory of epilepsy (i.e. preferred type of additional examination) could improve the lengthy and burdensome process which is often associated with the diagnosis of epilepsy. ${ }^{10}$ In addition, incorporating patients' preferences into medical decision-making has been shown to significantly impact their willingness to use healthcare services. ${ }^{28-30}$

Recent studies have however revealed a disparity between patients' preferences and those of physicians. ${ }^{60,61}$ For most conditions, physicians underestimated the impact of intervention characteristics on patients' decision making. ${ }^{61}$ Moreover, although it has been shown that a majority of physicians identified patient preferences as the most important general ethical guideline for surrogate decision-making, their medical decision-making relied on a variety of factors including prognosis, costs, patients' pain and suffering, and what was best for the patient overall (which closely resembles their preferences). ${ }^{62}$

In the Netherlands, the unit price of a MEG recording is about 200 Euros whereas the unit price of a $24 \mathrm{~h}-E E G$ or an EEGsd recording is about 160 Euros (price index year $2011) .{ }^{63}$ While the MEG recording is more expensive at first sight, the cost-effectiveness (i.e. value for money) of this modality in epilepsy diagnostics will highly depend on the quantity and quality of the data delivered by the MEG recording compared to the data of a $24 \mathrm{~h}-E E G$ and EEGsd recording. Although Colon et al. (2009) examined the additional value of MEG compared to EEGsd, future research should try to identify the informational value of the various diagnostic tests in relation to their financial implications as no studies have yet been performed on routine EEG, EEGsd, 24h-EEG and MEG in a head-to-head comparison. Furthermore, future research should try to identify sources of heterogeneity between epilepsy patients and the importance of incorporating these individual preferences in medical decision-making.

\section{Acknowledgements}

We would like to thank all respondents who completed the questionnaire, lab technicians and neurologists, especially Dr. M. Majoie and Drs. S. Ebus, for their participation in this study. Furthermore, we would like to thank L. Linssen and M. van Beers for their logistical support. 


\section{References}

1. Jacoby A, Baker GA. Quality-of-life trajectories in epilepsy: a review of the literature. Epilepsy Behav 2008; 12:557-71.

2. Taylor RS, Sander JW, Taylor RJ, Baker GA. Predictors of health-related quality of life and costs in adults with epilepsy: a systematic review. Epilepsia $2011 ; 52: 2168-80$.

3. Kotsopoulos IA, Evers SM, Ament AJ, de Krom MC. Estimating the costs of epilepsy: an international comparison of epilepsy cost studies. Epilepsia 2001;42:634-40.

4. Kotsopoulos IA, Evers SM, Ament AJ, Kessels FG, de Krom MC, Twellaar M, Metsemakers JF, Knottnerus AJ. The costs of epilepsy in three different populations of patients with epilepsy. Epilepsy Res 2003;54: 131-40.

5. Ngugi AK, Kariuki SM, Bottomley C, Kleinschmidt I, Sander JW, Newton CR. Incidence of epilepsy: a systematic review and meta-analysis. Neurology $2011 ; 77: 1005-12$.

6. Ngugi AK, Bottomley C, Kleinschmidt I, Sander JW, Newton CR. Estimation of the burden of active and life-time epilepsy: a meta-analytic approach. Epilepsia 2010;51:883-90.

7. Neligan A, Hauser WA, Sander JW. The epidemiology of the epilepsies. Handbook of clinical neurology / edited by P.J. Vinken and G.W. Bruyn 2012;107:113-33.

8. Smith D, Defalla BA, Chadwick DW. The misdiagnosis of epilepsy and the management of refractory epilepsy in a specialist clinic. QJM 1999;92:15-23.

9. Chadwick D, Smith D. The misdiagnosis of epilepsy. BMJ 2002;324:495-6.

10. Benbadis S. The differential diagnosis of epilepsy: a critical review. Epilepsy Behav 2009;15:15-21.

11. Evidence NIfC. NICE guidelines: the diagnosis and management of the epilepsies in adults and children in primary and secondary care. In: NICE, editor. London; 2012.

12. Network SIG. Diagnosis and management of epilepsy in adults. In. Edinburgh: SIGN; 2003.

13. Epilepsie NvvnNLt. Epilepsie; Richtlijnen voor diagnostiek en behandeling. Werkgroep Richtlijnen Epilepsie 2006.

14. Noachtar $S$, Remi J. The role of EEG in epilepsy: a critical review. Epilepsy Behav 2009; 15:22-33.

15. Flink R, Pedersen B, Guekht AB, Malmgren K, Michelucci R, Neville B, Pinto F, Stephani U, Ozkara C. Guidelines for the use of EEG methodology in the diagnosis of epilepsy. International League Against Epilepsy: commission report. Commission on European Affairs: Subcommission on European Guidelines. Acta neurologica Scandinavica 2002; 106:1-7.

16. Rajna $P$, Veres J. Correlations between night sleep duration and seizure frequency in temporal lobe epilepsy. Epilepsia 1993;34:574-9.

17. Scalise A, Desiato MT, Gigli GL, Romigi A, Tombini M, Marciani MG, Izzi F, Placidi F. Increasing cortical excitability: a possible explanation for the proconvulsant role of sleep deprivation. Sleep 2006;29: 1595-8.

18. Matos G, Andersen ML, do Valle AC, Tufik S. The relationship between sleep and epilepsy: Evidence from clinical trials and animal models. J Neurol Sci 2010;295:1-7.

19. Frucht MM, Quigg M, Schwaner C, Fountain NB. Distribution of seizure precipitants among epilepsy syndromes. Epilepsia 2000;41:1534-9.

20. Malow BA. Sleep deprivation and epilepsy. Epilepsy Curr 2004;4:193-5.

21. Nakken KO, Refsland G, Lillestolen KM, Solaas MH. Seizure-precipitating factors in epilepsy--what do patients report? Tidsskr Nor Laegeforen 2005; 125:2172-4.

22. Colon AJ, Ossenblok P, Nieuwenhuis L, Stam KJ, Boon P. Use of routine MEG in the primary diagnostic process of epilepsy. J Clin Neurophysiol 2009;26:326-32.

23. Heers M, Rampp S, Kaltenhauser M, Pauli E, Rauch C, Dolken MT, Stefan H. Detection of epileptic spikes by magnetoencephalography and electroencephalography after sleep deprivation. Seizure 2010;19: 397-403.

24. Ossenblok P, de Munck JC, Colon A, Drolsbach W, Boon P. Magnetoencephalography is more successful for screening and localizing frontal lobe epilepsy than electroencephalography. Epilepsia 2007;48: $2139-49$.

25. Bridges JF, Jones C. Patient-based health technology assessment: a vision of the future. Int J Technol Assess Health Care 2007; $23: 30-5$. 
26. Facey K, Boivin A, Gracia J, Hansen HP, Lo Scalzo A, Mossman J, Single A. Patients' perspectives in health technology assessment: a route to robust evidence and fair deliberation. Int J Technol Assess Health Care 2010;26:334-40.

27. Ryan M. Discrete choice experiments in health care. BMJ 2004;328:360-1.

28. Dominitz JA, Provenzale D. Patient preferences and quality of life associated with colorectal cancer screening. Am J Gastroenterol 1997;92:2171-8.

29. Marshall DA, Johnson FR, Kulin NA, Ozdemir S, Walsh JM, Marshall JK, Van Bebber S, Phillips KA. How do physician assessments of patient preferences for colorectal cancer screening tests differ from actua preferences? A comparison in Canada and the United States using a stated-choice survey. Health Econ 2009; 18:1420-39.

30. Woolf SH. The best screening test for colorectal cancer--a personal choice. N Engl J Med 2000;343: 1641-3.

31. Lancsar $E$, Louviere J. Conducting discrete choice experiments to inform healthcare decision making: a user's guide. PharmacoEconomics 2008;26: 661-77.

32. Ryan M, Gerard K. Using discrete choice experiments to value health care programmes: current practice and future research reflections. Appl Health Econ Health Policy 2003;2:55-64.

33. Essers BA, Dirksen CD, Prins MH, Neumann HA. Assessing the public's preference for surgical treatment of primary basal cell carcinoma: a discrete-choice experiment in the south of the Netherlands. Dermatol Surg 2010;36:1950-5.

34. Hol L, de Bekker-Grob EW, van Dam L, Donkers B, Kuipers EJ, Habbema JD, Steyerberg EW, van Leerdam ME, Essink-Bot ML. Preferences for colorectal cancer screening strategies: a discrete choice experiment. Br J Cancer 2010; 102:972-80.

35. Paczkowski MM, Kruk ME, Tessema F, Tegegn A, Galea S. Depressive symptoms and posttraumatic stress disorder as determinants of preference weights for attributes of obstetric care among Ethiopian women. PloS One 2012;7:e46788.

36. Regier DA, Diorio C, Ethier MC, Alli A, Alexander S, Boydell KM, Gassas A, Taylor J, Kellow C, Mills D, Sung L. Discrete choice experiment to evaluate factors that influence preferences for antibiotic prophylaxis in pediatric oncology. PloS One 2012;7:e47470.

37. Manjunath R, Yang J-C, Ettinger AB. Patients' preferences for treatment outcomes of add-on antiepileptic drugs: A conjoint analysis. Epilepsy Behav 2012;24(4):474-9.

38. de Bekker-Grob EW, Hol L, Donkers B, van Dam L, Habbema JDF, van Leerdam ME, Kuipers EJ, Essink-Bot M-L, Steyerberg EW. Labeled versus unlabeled discrete choice experiments in health economics: an application to colorectal cancer screening. Value Health 2010;13:315-323.

39. Ryan M, Farrar S. Using conjoint analysis to elicit preferences for health care. Bmi 2000;320:1530-3.

40. Louviere JJ, Hensher DA, Swait JD. Stated choice methods: analysis and applications: Cambridge University Press; 2000.

41. Bryan S, Dolan P. Discrete choice experiments in health economics. For better or for worse? Eur J Health Econ 2004; 5: 199-202.

42. Electrical Geodesics I. HydroCel Geodesic Sensor Nets http://www.egi.com/research-division-researchproducts/sensor-nets. In.

43. Binnie CD, Stefan H. Modern electroencephalography: its role in epilepsy management. Clin Neurophysiol 1999; 110:1671-97.

44. Walter WG, Hodge R, Hutchinson J. Discussion on recent advances in the EEG diagnosis of epilepsy. Proc R Soc Med 1951;44:315.

45. Reed Johnson F, Lancsar E, Marshall D, Kilambi V, Muhlbacher A, Regier DA, Bresnahan BW, Kanninen B, Bridges JF. Constructing experimental designs for discrete-choice experiments: report of the ISPOR Conjoint Analysis Experimental Design Good Research Practices Task Force. Value 2013;16:3-13.

46. Hiligsmann M, Dellaert 2 BG, Dirksen CD, van der Weijden T, S. G, Reginster J, Watson V, Boonen A. Patients' preferences for osteoporosis drug treatment: a discrete choice experiment. In. manuscript presented at the 5e LolaHESG conference, 23-24 May 2013, Landgoed Vennendal (the Netherlands) 2013.

47. Kessels R, Jones B, Goos P, Vandebroek M. The usefulness of Bayesian optimal designs for discrete choice experiments. Applied Stochastic Models in Business and Industry 201 1;27:173-188. 
48. Alayli-Goebbels AF, Dellaert BG, Knox SA, Ament AJ, Lakerveld J, Bot SD, Nijpels G, Severens J. Consumer Preferences for Health and Nonhealth Outcomes of Health Promotion: Results from a Discrete Choice Experiment. Value Health 2013;16:114-123.

49. Ryan M, Skatun D. Modelling non-demanders in choice experiments. Health Econ 2004; 13:397-402.

50. Bech $M$, Kjaer $T$, Lauridsen J. Does the number of choice sets matter? Results from a web survey applying a discrete choice experiment. Health Econ $2011 ; 20: 273-86$.

51. Bridges JF, Hauber AB, Marshall D, Lloyd A, Prosser LA, Regier DA, Johnson FR, Mauskopf J. Conjoint analysis applications in health--a checklist: a report of the ISPOR Good Research Practices for Conjoint Analysis Task Force. Value Health $2011 ; 14: 403-13$.

52. Hensher DA, Rose JM, Greene WH. Applied Choice Analysis: A Primer. Cambridge University Press 2005.

53. Malhotra NK, Birks DF. Marketing Research: An Applied Approach: Pearson Education, Limited; 2007.

54. Marshall DA, Johnson FR, Phillips KA, Marshall JK, Thabane L, Kulin NA. Measuring patient preferences for colorectal cancer screening using a choice-format survey. Value Health 2007; 10:415-30.

55. Nayaradou M, Berchi C, Dejardin O, Launoy G. Eliciting population preferences for mass colorectal cancer screening organization. Med Decis Making 2010;30:224-33.

56. Asch DA, Jedrziewski MK, Christakis NA. Response rates to mail surveys published in medical journals. J Clin Epidemiol 1997;50:1 129-1136.

57. van Helvoort-Postulart D, van der Weijden T, Dellaert BG, de Kok M, von Meyenfeldt MF, Dirksen CD. Investigating the complementary value of discrete choice experiments for the evaluation of barriers and facilitators in implementation research: a questionnaire survey. Implement Sci 2009;4:10.

58. Ryan M. Using conjoint analysis to take account of patient preferences and go beyond health outcomes: an application to in vitro fertilisation. Social science \& medicine 1999;48:535-46.

59. Helmstaedter C, Kurthen M, Lux S, Reuber M, Elger CE. Chronic epilepsy and cognition: a longitudinal study in temporal lobe epilepsy. Ann Neurol 2003;54:425-32.

60. Montgomery A, Fahey T. How do patients' treatment preferences compare with those of clinicians? Quality in Health Care 2001; 10:i39-i43.

61. Mühlbacher AC, Juhnke C. Patient Preferences Versus Physicians' Judgement: Does it Make a Difference in Healthcare Decision Making? Appl Health Econ Health Policy 2013:1-18.

62. Torke AM, Moloney R, Siegler M, Abalos A, Alexander GC. Physicians' Views on the Importance of Patient Preferences in Surrogate Decision-Making. J Am Geriatr Soc 2010;58:533-538.

63. The Dutch Healthcare Authority. DBC zorgproducten tariefapplicatie. http://dbc-zorgproductentarieven.nza.nl/nzaZpTarief/Welkom.aspx. 2013. 



\section{From clinically relevant}

\section{outcome measures to quality}

of life in epilepsy

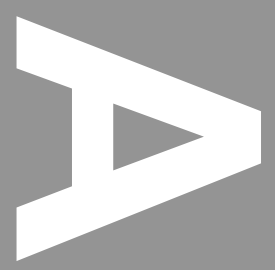

A time trade-off study
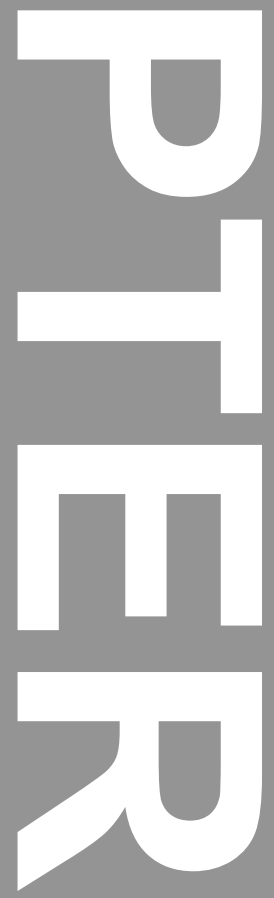

R.J.A. de Kinderen

B.F.M. Wiinen

G. van Breukelen

D. Postulart

M.H.J.M. Majoie

A.P. Aldenkamp

C.D. Dirksen

S.M.A.A. Evers

Epilepsy Res, 2016;25:24-31

*This study has been part of the PhD thesis of dr. R.J.A. de Kinderen 


\section{Abstract}

\section{Objective}

A proposed method for bridging the gap between clinically relevant epilepsy outcome measures and quality-adjusted life years is to derive utility scores for epilepsy health states. The aim of this study is to develop such a utilityfunction and to investigate the impact of the epilepsy outcome measures on utility.

\section{Methods}

Health states, based on clinically important epilepsy attributes (e.g. seizure frequency, seizure severity, side-effects), were valued by a sample of the Dutch population ( $N=525)$ based on the time trade-off method. In addition to standard demographics, every participant was asked to rate 10 or 11 different health state scenarios. A multilevel regression analysis was performed to account for the nested structure of the data.

\section{Results}

Results show that the best health state (no seizures and no side-effects) is estimated at 0.89 and the worst state (seizures type 5 twice a day plus severe side-effects) at 0.22 (scale: 0 1). An increase in seizure frequency, occurrence of side-effects, and seizure severity were all significantly associated with lower utility values. Furthermore, seizure severity has the largest impact on quality of life compared with seizure frequency and side-effects.

\section{Conclusions}

This study provides a utility-function for transforming clinically relevant epilepsy outcome measures into utility estimates. We advise using our utility-function in economic evaluations, when quality of life is not directly measured in a study and hence, no health state utilities are available, or when there is convincing empirical evidence of the insensitivity of a generic quality-of-life-instrument within epilepsy. 


\section{Introduction}

Quality of life (QoL) is a very important outcome measure, not only for patients, but also as an input for cost-utility analyses. A cost-utility analysis is a form of economic evaluation in which costs and benefits of alternative interventions are compared to see whether the investigated intervention offers good 'value for money'. The benefits of a cost-utility analysis are expressed in Quality Adjusted Life Years (QALYs), which incorporate effects in terms of both QoL (utilities) and survival (life-years gained). The value a person assigns to a particular health state (i.e. description of a particular set of symptoms which is common to a particular disease) is commonly referred to as a utility, and lies on a scale where death and full health are assigned values of 0 and 1, respectively. Using utility values one can calculate QALYs by multiplying the numbers of life-years gained by the utility of those added life-years. However, the essential utilities are not always available. Especially in case of model-based, cost-utility analyses, where researchers are dependent on the published literature, suitable utilities for certain health states are often hard to find.

Obviously, as utilities are used to calculate QALYs, they need to be measured in a methodologically sound and preferably uniform manner. Hence, the ISPOR Task force regarding the measurement of utilities, called for the use of direct utility elicitation methods, such as the standard gamble (SG) or time trade-off (TTO) exercises. ${ }^{1}$

Problems in gathering utility values may occur because clinical trials and clinical evaluations measure the effects of interventions with common clinically relevant measures, rather than with QoL-measures.

Within the field of epilepsy, three disease-specific clinical outcome measures are often used to reflect patients' health, namely; seizure frequency, seizure severity and side-effects due to antiepileptic drugs (AEDs). Frequency and severity of seizures in people with epilepsy vary from one individual to another. In some people, seizures are very severe and occur frequently despite treatment with AEDs. In others, seizures are mild, less frequent and more easily controlled by AEDs. Seizure severity is measured using standardized questionnaires. One of the most frequently used seizure severity questionnaires focusing on the clinical events of a seizure, is the National Hospital Seizure Severity Scale (NHS3), a revised and simplified version of the Chalfont scale. ${ }^{2}$

In order to bridge the gap between clinical trials, which measure clinical outcomes, and economic evaluations, which require utility values, it would be preferable if one could make clinically relevant epilepsy outcome measures applicable to cost-utility analysis by deriving utility scores for the epilepsy outcome measures. For this purpose, the TTO method was used, in which participants are asked to determine how many life years one is willing to give up in order to avoid a particular health state. ${ }^{3}$ This method has been used before in four other studies which elicited utilities for epilepsy health states. Firstly, Messori et al. (1998) interviewed a small series of refractory epilepsy patients $(N=81)$ and asked them how much of their current state of health they would be willing to give up in order to live the remaining years in excellent health. ${ }^{4}$ Secondly, Forbes et al. (2003) executed a TTO experiment among a smaller group of epilepsy patients $(\mathrm{N}=43) .{ }^{5}$ However, only seven 
patients understood the exercise. ${ }^{5}$ Thirdly, Carroll and Downs (2009) interviewed a large group of parents $(\mathrm{N}=4016)$ to obtain their assessment of various health states while imagining that it would be one of their children who was experiencing the health state under consideration. ${ }^{6}$ Lastly, Kang et al. (2014) asked the general population ( $N=300$ ) to assess three epilepsy health states. ${ }^{7}$ These published TTO-studies elicited utilities for a very small number of specific health states (i.e. 1-5); furthermore, in these studies, seizure severity and side-effects were not (fully) included in the health state description.

To overcome this gap, the objective of this study is twofold: first of all, to create a reliable utility-function to transfer clinically relevant epilepsy outcomes to health state utilities based on preferences of the general public measured with the TTO method, and secondly, to investigate the impact of the separate clinically relevant outcome measures on health state utility. Preferences of the general public are used in economic evaluations as policy decisions are often made on societal level. Hence, it is stated within health economics, that the population potentially affected by resource allocation decision should be polled, and that the general public is supposed to value policy decisions with the most benefit for society as a whole. ${ }^{8}$ In addition it has been demonstrated that 'outsiders' may be more able to differentiate across treatment groups than patients are themselves.

\section{Methods}

\subsection{Study participants}

Participants from the general population aged $>18$ years without epilepsy were recruited using e-mail, personal communication and social media. A link was provided to participants, which gave access to the online questionnaire. As part of this communication, we asked the participants to forward our invitation to participate in the study to their family and friends (again aged $>18$, without epilepsy). Participants who did not fully complete the questionnaire were excluded from the analyses. Participants were told that if they decided to complete the questionnaire, they thereby consented to participate in the study. In addition, information was provided that participation, and/or forwarding the invitation to friends and family, was completely voluntary and anonymous. This consent procedure was approved by the medical ethical committee of the epilepsy center Kempenhaeghe.

\subsection{Attributes and levels}

Health states were developed based on three clinically important disease-specific outcome measures (i.e. attributes): seizure frequency, seizure severity and treatment-related sideeffects. These attributes were selected based on expert advice and commonality of use within the clinical epilepsy setting. Table 3.1 presents the three attributes and their levels. The attribute "seizure frequency" comprises 6 levels ranging from no seizures to two seizures per day. The levels of "seizure severity" were based on the description of seizures 
used in the valuation study of the National Hospital Seizure Severity Scale-3 (NHS3). ${ }^{2}$ The attribute "experience of side-effects" was categorized into three levels: no or mild, moderate and severe side-effects. Figure 3.1 presents an example of a health state to be valued by participants.

Table 3.1 Attributes and levels for TTO exercise

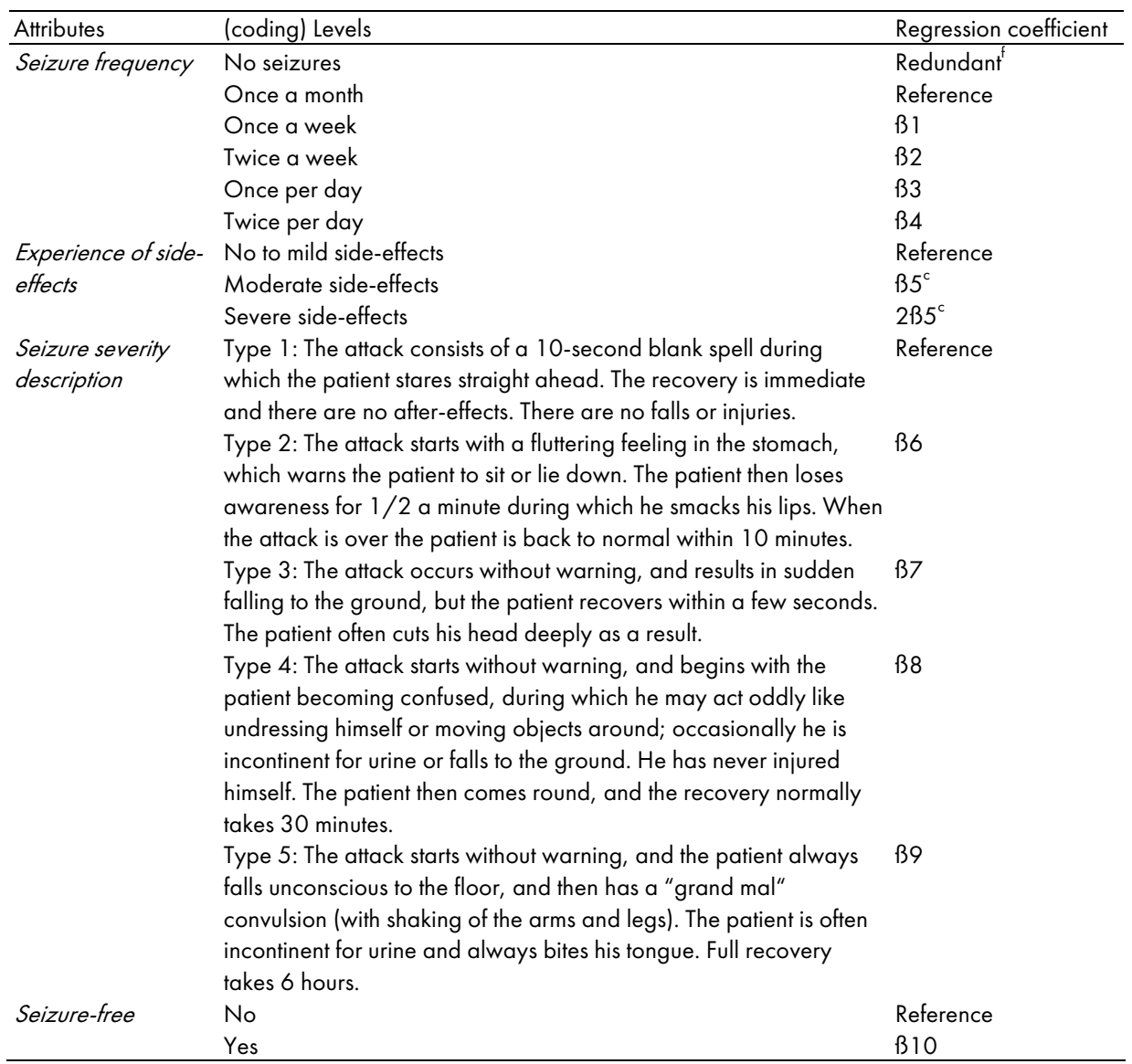

"Level "No seizures" is captured by the extra dummy indicator "Seizure-free" coded as 0, not seizure-free; 1, seizure-free. ${ }^{c}$ Included in the model as a continuous variable

Seizure frequency:
You will experience one seizure a day
Side-effects:
You will experience moderate side-effects
Seizure severity:
The attack occurs without warning, and results in sudden
falling to the ground, but you will recover within a few
seconds. You will often cut your head deeply as a result.

Figure 3.1 Example of a health state description 


\subsection{Valuation exercise}

Preferences of a sample of the general population were elicited through a time trade-off (TTO) exercise for each of the epilepsy health states. The TTO was based on a process of outward titration to select a length of time in a health state with full health which they regarded as being equivalent to 10 years in an epilepsy health state. In other words; participants were asked which life they thought would be better, life A or life B (or are the two equivalent)? Life $A$ : living for 10 years within the presented epilepsy health state; life $B$ : living $t=5$ years in perfect health. In the case that the participant prefers life $A$ to life $B, t$ is increased by 1 point (maximum $t=10$ ), until the participant becomes indifferent to whether it is life $A$ or life $B$. The other way around, if a participant prefers life $B$ to life $A, t$ is decreased (minimum $t=0$ ), until the participant becomes indifferent to whether it is life $A$ or life $B$. The smaller the 'equivalent' number ' $t$ ', the worse the epilepsy health state. If a respondent was willing to sacrifice all t years in full health to avoid a certain epilepsy health state, then the participant valued the epilepsy health state as being equivalent to death.

A (nearly) full factorial design was used which resulted, after removing all unrealistic scenario's (i.e. all combinations of "no seizures" with "seizure type"), in 78 different epilepsy health states. Each participant valued a subset of 10 or 11 epilepsy health states, comprising the mildest possible health state (no seizures and no to mild side-effects), the worst possible health state (i.e. two seizures per day, severe side-effects and seizure description 5) and a random subset of 8 or 9 relatively mild, moderate and severe health states (until saturation was reached i.e. 50 values per health state).

\subsection{Questionnaire}

The questionnaire was self-administered using online survey software Qualtrics, version 2013 (Qualtrics, Provo, UT, USA). The first part of the online questionnaire consisted of socio-economic and background questions/characteristics: age, gender, educational level and experience with epilepsy (e.g. friend or family with epilepsy). Furthermore, we asked patients whether they believe in life after death, as this might influence their willingness to trade-off life years in the valuating exercise. ${ }^{10}$

Lastly, participants were asked to describe their own QoL using the EuroQol-5 Dimensions (EQ-5D) ${ }^{11}$ including a Visual Analogue Scale (VAS). The second part of the questionnaire started with background information about epilepsy and quality of life. Next, the attributes and levels of epilepsy health states were explained and some example questions were shown as a warm-up exercise for the TTO. The third and final part of the questionnaire consisted of the TTO exercises and questions about the time required by the participants to complete the questionnaire and their experience regarding the difficulty of the TTO exercise. 


\subsection{Analyses}

Utilities (U) were derived by determining the number of life years one is willing to sacrifice in order to become indifferent with regard to the choice between the epilepsy health state $(\mathrm{H})$ scenario $(\mathrm{j})$ to be valued and t years spent in full health. Afterwards, $U$ is determined as $U\left(H_{i}\right)=t / 10$, where $t$ is the time in full health.

To account for the nested structure of the data (i.e. each respondent evaluated 10 or 11 scenarios), multilevel analyses were performed to develop the best model for the effects of the three attributes (seizure frequency, seizure severity, side-effects) on utility. Thereby, multilevel analyses were used to develop the utility-function, and to determine the effect of other covariates on the health state valuation of the respondents. We started with a random intercept model to capture inter-individual differences plus an ARMA auto-correlated errors model for the unexplained intra-individual response variation throughout the trials. To find the best model for the attribute effects on the utility, several models were compared by likelihood ratio (LR) testing, using maximum likelihood (ML) estimation. ML estimation is required for $L R$ tests of models differing in their fixed/predictor part. ${ }^{12}$ However, the final model was re-estimated using restricted maximum likelihood (REML) estimation to obtain unbiased estimates of standard errors. ${ }^{12}$

Subsequently, to determine whether participants' religious belief, age, gender, educational level, experience with epilepsy (i.e. a close relative or a friend with epilepsy) and current QoL (EQ-5D and VAS score) significantly impacted the valuation exercise, they were included in the model as covariates. Multicollinearity of the covariates was assessed by checking if the Variance Inflation Factor (VIF) was below 10 for every covariate.

\subsection{Additional analyses}

We used the same model for the additional analyses, but now incorporating the utility values derived with the lead time trade-off. ${ }^{13}$ Only when participants traded off all 10 healthy life years to avoid a certain health state, they were offered the possibility of valuing the health state as being worse than death (SWD). In this case, the same scenario was presented using the lead time trade-off approach in which the respondent was asked to select a length of time $(t)$ for which one is indifferent between spending $t$ years in full health or spending 5 years in full health followed by 10 years in the given health state $\left(\mathrm{H}_{\mathrm{i}}\right)$. The corresponding utility is then estimated as $U\left(H_{i}\right)=(t-5) /(15-5)$, resulting in a range of 0.5 to 0 for states worse than death.

\section{Results}

\subsection{Background characteristics}

In total, 529 subjects from the general population participated in the TTO study. Four participants were excluded from the analyses as their data were incomplete due to a 
computer error. Therefore, a total of 525 participants were included in the analyses. The mean age of the included sample was 43 years; more than half $(59.5 \%)$ were women; most of them had an educational level of at least secondary vocational education $(86.1 \%)$ and $61.9 \%$ had had no experience with epilepsy whatsoever. They valued their own health state, based on the EQ-5D at 0.86 (Dutch tariffs), ${ }^{10} 0.84$ (UK tariffs) ${ }^{14}$ and 84.7 (VAS). Of the included participants, $37 \%$ indicated that they believed in life after death (Table 3.2).

Table 3.2 Respondent characteristics

\begin{tabular}{|c|c|c|}
\hline Characteristics & $\begin{array}{c}\text { Total cohort \% } \\
(\mathrm{N}=525)\end{array}$ & $\begin{array}{c}\text { General Dutch } \\
\text { population }\end{array}$ \\
\hline \multicolumn{3}{|l|}{ Gender } \\
\hline Male & 40.5 & 49.5 \\
\hline Female & 59.5 & 50.5 \\
\hline \multicolumn{3}{|l|}{ Age in years } \\
\hline $20-40$ & $44.9^{\mathrm{i}}$ & 24.6 \\
\hline $40-65$ & 45.4 & 35.5 \\
\hline $65-80$ & 9.7 & 12.6 \\
\hline $80+$ & 0 & 4.2 \\
\hline \multicolumn{3}{|l|}{ Education } \\
\hline No education & 0.4 & 1 \\
\hline Primary school & 1.3 & 8 \\
\hline Pre-vocational secondary school & 12.2 & 19 \\
\hline Secondary school ${ }^{c}$ & 30.2 & 40 \\
\hline Higher education $^{d}$ & 55.9 & 32 \\
\hline \multicolumn{3}{|l|}{ Experience with epilepsy } \\
\hline No experience & 61.9 & N.a. \\
\hline Relative with epilepsy & 10.1 & N.a. \\
\hline Friend with epilepsy & 4.8 & N.a. \\
\hline Former epilepsy patient & 0.8 & N.a. \\
\hline Other experience ${ }^{e}$ & 22.5 & N.a. \\
\hline \multicolumn{3}{|l|}{ Believes in life after death } \\
\hline Yes & 37.0 & N.a. \\
\hline No & 63.0 & N.a. \\
\hline \multicolumn{3}{|l|}{ Mean EuroQol-5D score } \\
\hline Dutch tariffs & 0.86 & $0.91^{f}$ \\
\hline UK tariffs & 0.84 & $0.89^{f}$ \\
\hline Visual analogue scale & 84.7 & $87.4^{f}$ \\
\hline
\end{tabular}

N.a., Not available. ${ }^{i}$ Includes 17 respondents aged $18-20 ;{ }^{c}$ Includes: General vocational school, pre-university school and secondary vocational education ${ }^{\mathrm{d}}$ Includes: Professional and academic higher education; ${ }^{\mathrm{e}}$ Includes other ways of having experience with epilepsy (e.g. schoolmate with epilepsy, a pet with epilepsy etc.); ${ }^{f}$ Based on Bernert et al. ${ }^{20} ;$ "f Based on Statistics Netherlands (CBS, 2013)

\subsection{Health state valuation}

We started with an initial model with a random intercept to capture inter-individual differences plus an ARMA model for the unexplained intra-individual response variation over trials. In this analysis we treated all three attributes as categorical variables to allow any kind of relationship between independent and dependent variables. The categories 
"one seizure per month", "no to mild side-effects" and "type I seizure" were used as reference categories for, respectively, "seizure frequency", "experience of side-effects" and "seizure severity", and a dummy indicator was included into the model for every other category of each attribute, plus one extra dummy indicator for absence of seizures (to distinguish between absence of seizures and the mildest epilepsy health state, i.e. a type 1 seizure once a month).

Then we compared the model fit with that of a simplified model in which all attributes were included in the model as covariates, thus assuming a linear effect of each attribute on the health state evaluation. This resulted in a significantly worse model fit (Likelihood Ratio test: $\left.\chi^{2}=53.823, \mathrm{df}=7, \mathrm{p}<0.005\right)$. However, treating only the attribute "experience of sideeffects" as a covariate gave a model fit comparable with that of the initial categorical model $\left(\chi^{2}=0.054, d f=1, p>0.05\right)$. Next, we allowed the effect of the covariate "experience of side-effects" to vary randomly between participants, and this random slope was allowed to covary with the random intercept. This resulted in a significantly better model fit $\left(\chi^{2}=46.039, d f=1, p<0.005\right)$. Finally, we included interactions between all attributes and then reduced the model by deleting non-significant interactions. This resulted in a model with an interaction between the attributes "seizure frequency" and "seizure severity", which fitted better than the model without interaction $\left(\chi^{2}=65.213, \mathrm{df}=16\right.$, $\mathrm{p}<0.005)$, but at the cost of sixteen additional parameters $(4 * 4)$. Compared to the plotted mean observed utility values (Figure $3.2 a$ ), the plotted fixed predicted values of this model (Figure 3.2b) look very similar. To investigate whether the simplified model without interaction is also acceptable, although strictly speaking significantly worse (which may be due to the large sample size), the fixed predicted values of the model without interaction are plotted in Figure 3.2c. This shows that this model is also quite acceptable.

Although Figure 3.2 suggests that the model can be even further simplified by treating the attributes "seizure frequency" and "seizure severity" as continuous covariates and thus assuming their effects to be linear, this model was significantly worse than the model behind Figure $3.2 \mathrm{c}\left(\chi^{2}=55.87, \mathrm{df}=6, \mathrm{p}<0.005\right)$. In relation to the above, it is clinically more relevant to address the attribute "seizure severity" as a categorical rather than continuous predictor, because the successive seizure severity types do not look equidistant clinically, ${ }^{2}$ and the attribute "seizure frequency" was not measured on an equidistant scale.

Finally, all other covariates were put into the model (there was no collinearity, i.e. all $\mathrm{VIF}<10$ ). Participants' gender, religious belief, educational level, experience with epilepsy and current QoL (EQ-5D and VAS) did not impact the valuation exercise significantly (all $p>0.10)$. Age was the only characteristic which significantly influenced the health state valuation. The older the participant, the more negative the valuation of the health states. This age effect was, however, very small (i.e. 0.001 per year) and the regression coefficients of the attributes hardly changed when age was added to the model. The final model is, therefore, a model with a random intercept, in which "seizure frequency", "seizure severity" and "seizure-free" are categorical predictors, "experience of sideeffects" is a continuous predictor with a random slope, and in which no interactions or other covariates are included (Table 3.3). 


$$
\begin{aligned}
& \text { a } \\
& \text { a }
\end{aligned}
$$
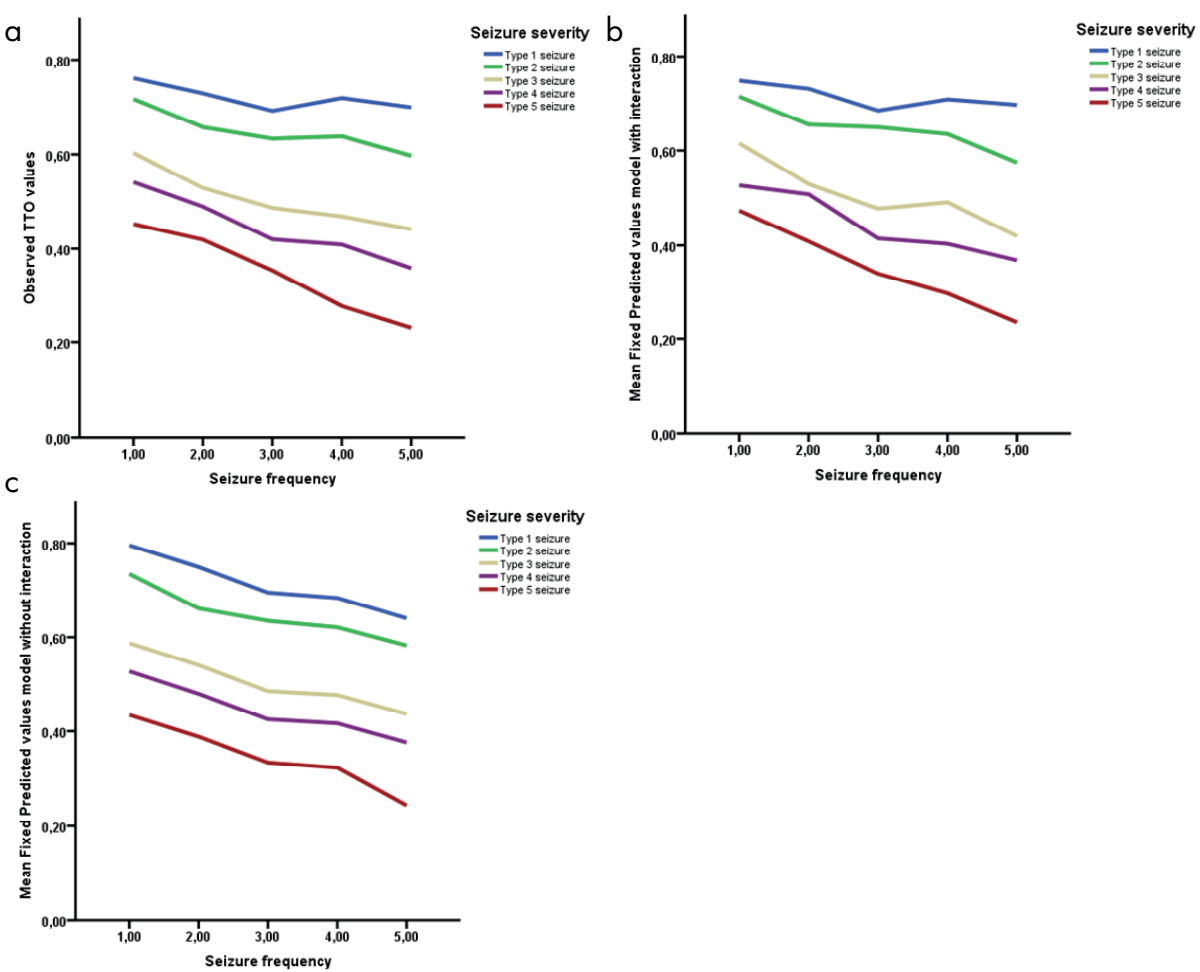

Seizure severity

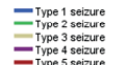

Figure 3.2 a) Plotted mean observed utility values, b) plotted fixed predicted utility values with interaction, and c) plotted fixed predicted utility values without interaction

All attributes significantly affected patients' health state valuation $(p<0.05)$. Furthermore, the coefficient of the indicator "seizure-free" represents the increase in health state value of being seizure-free as compared to having a type 1 seizure once a month (i.e. reference category), keeping side-effects the same.

Finally, the constant term can be interpreted as the average utility for a patient with one seizure per month of seizure type 1 and who experiences no to mild side-effects. The utilityfunction, including example utility calculations for three hypothetical health states, can be found in Figure 3.3. Furthermore, when looking at the total range of impact of each attribute (i.e. difference between "best" level and "worst" level), these results indicate that "seizure type" has a substantially larger maximum effect on health state valuation than "seizure frequency" and "experience of side-effects", and that the latter two attributes have almost the same maximum effect (bearing in mind the coding of experience of side-effects). 
Table 3.3 Results from multilevel analysis illustrating influence of variables on health state valuation in the final model ( $t$ between 0 to 10 ) and for the additional model (SWD, t between -5 to 10 )

\begin{tabular}{|c|c|c|c|c|c|c|}
\hline \multirow[b]{2}{*}{ Variable } & \multicolumn{3}{|c|}{ Final model } & \multicolumn{3}{|c|}{ Additional model } \\
\hline & Coefficient & SE & Sign. & Coefficient & $\mathrm{SE}$ & Sign. \\
\hline Intercept & .857 & .023 & .000 & .861 & .013 & .000 \\
\hline \multicolumn{7}{|l|}{ Seizure frequency } \\
\hline \multicolumn{7}{|l|}{ Once a month $^{f}$} \\
\hline Once a week & -.045 & .009 & .000 & -.053 & .010 & .000 \\
\hline Twice a week & -.102 & .009 & .000 & -.110 & .010 & .000 \\
\hline Once per day & -.113 & .009 & .000 & -.130 & .010 & .000 \\
\hline Twice per day & -.150 & .008 & .000 & -.171 & .009 & .000 \\
\hline Experience of side-effects ${ }^{c}$ & -.061 & .004 & .000 & -.068 & .004 & .000 \\
\hline \multicolumn{7}{|l|}{ Seizure severity description } \\
\hline \multicolumn{7}{|l|}{ Seizure type $1^{\dagger}$} \\
\hline Seizure type 2 & -.060 & .009 & .000 & -.058 & .010 & .000 \\
\hline Seizure type 3 & -.208 & .009 & .000 & -.216 & .010 & .000 \\
\hline Seizure type 4 & -.273 & .009 & .000 & -.290 & .010 & .000 \\
\hline Seizure type 5 & -.361 & .008 & .000 & -.391 & .009 & .000 \\
\hline Seizure-free ${ }^{d}$ & .037 & .011 & .001 & .028 & .013 & .024 \\
\hline
\end{tabular}

SWD, States worse than death; SE, Standard error; Sign., Significance. ${ }^{\circ}$ Experience of side-effects is coded as 0 , no to mild side-effects; 1 , moderate side-effects; 2 , severe side-effects; ${ }^{d}$ Seizure-free is coded as 0 , no; 1 , yes Note: Both models contain no interactions or other covariates; the random part consists of a random intercept plus random slope of the attribute "experience of side-effects" (with intercept-slope covariance) for the betweensubject part, and ARMA 11 for the within-subject part, of the covariance pattern; the results are based on REML estimation. ${ }^{f}$ Reference category

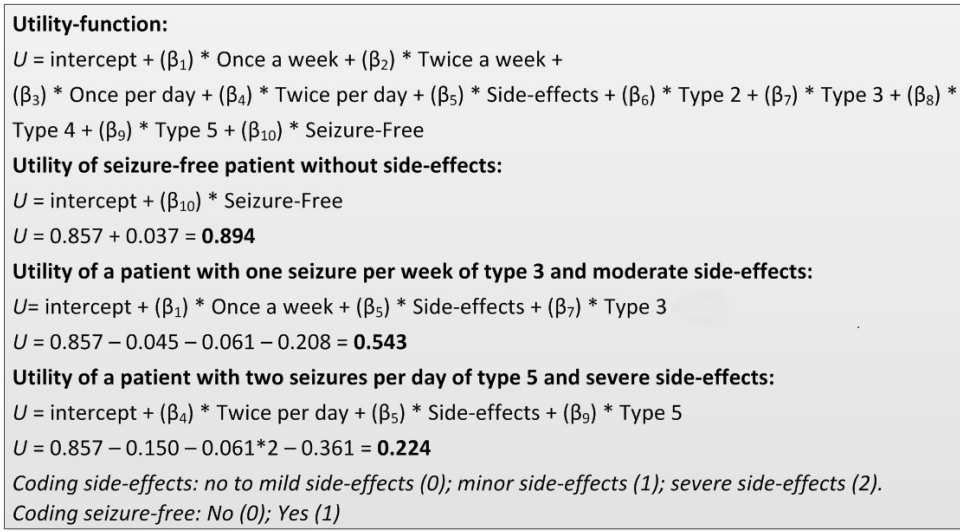

Figure 3.3 Utility-function and examples of utility calculations for three health states

\subsection{Additional analyses}

Table 3.3 also includes the results from the random effects model illustrating the influence of variables on health state valuation when allowing participants to value SWD. The inclusion of SWD results in a slightly higher constant term, and all regression coefficients 
become larger in absolute terms except for the coefficient, seizure-free. This seems logical as the limit of valuation of a health state in the lead TTO was not 0 , but -0.5 , as lower boundary.

\section{Discussion}

This study has provided utility values for 78 health states based on three clinically important epilepsy attributes (e.g. seizure frequency, seizure severity, side-effects). The results suggest a utility-function that could be used when health states, described by seizure frequency, seizure severity and side-effects are available to predict a utility score. In short, our study shows that seizure frequency, seizure severity and side-effects have a significant impact on QoL. However, looking at the results more closely, this study clearly demonstrates that seizure severity has a greater negative impact on utility compared with the attributes seizure frequency and side-effects.

It is important that utility values are accurately assessed. In the absence of accurate values, researchers performing cost-utility analyses are often forced to guess the utility values for disease states, possibly leading to extreme variability between studies and questionable results. This study provides a utility-function to transform multiple epilepsy health states into utilities valued by a fairly large sample of the general Dutch population and elicited using established methods. As a result this utility-function is suitable for economic evaluations.

This study has some limitations that deserve consideration. Firstly, our sample was a convenience sample, not fully representative of the general Dutch population. The sample consisted of slightly more women, was relatively young, quite highly educated, and their own quality of life was somewhat lower than that of the general Dutch population. Furthermore, the sample had a variety of experience with epilepsy which can also bias the results. Secondly, the TTO exercise was conducted via a computerized outward titration method. Although participants could consult the researcher via email or telephone in case of questions/difficulties, there was no interviewer present during the computerized exercise. Most participants (88.2\%) indicated that they had understood the exercise; however, only $46.1 \%$ said the questions were easy. Participants indicated that the health descriptions were clear $(87.3 \%)$ but that it was sometimes difficult to make a choice between the health states $(43.8 \%)$, or to become indifferent between the described epilepsy health state and a shorter health state in perfect health (45.9\%). Most of the participants $(88 \%)$ needed less than 30 minutes to complete the TTO exercise; only a small number of patients $(0.6 \%)$ needed 1.5 hours. Finally, although most participants indicated that they had understood the exercise, potential misunderstanding can lead to biased results.

Large differences are found between studies presenting utility values for epilepsy health states. $^{4-7}$ This is due to differences in health state descriptions, the sample used to value health states (e.g. patients or general population), and the elicitation method used. ${ }^{15}$ The 
latter issue, is for example also present in our study, although not surprisingly, the coefficients measured with the 'normal' TTO differed from those measured with the 'Leadtime' TTO.

Obviously, as utilities are used to calculate QALYs necessary for economic evaluations, they need to be measured in a methodologically sound and preferably uniform manner. The recommendation outlined by the ISPOR RCT-CEA Task force regarding the measurement of utilities, called for the use of generic preference-based outcome instruments, such as the EuroQol (EQ-5D), or for direct utility elicitation methods, such as the standard gamble or time trade-off exercises. 'Despite the growing use of generic preference-based outcome instruments such as the EQ-5D, there are many clinical studies, which have recorded outcome information only in the form of non-preference-based instruments. Likewise, researchers working in the field of epilepsy are primarily concerned with measuring symptoms, disease progression and disease-specific QoL and the instruments to do this are generally referred to by economists as non-preference-based measures. On the one hand, using clinically relevant epilepsy outcome measures to calculate utilities, as is done in this study, may have the merit of sensitivity to diseasespecific effects of interventions. On the other hand, the loss of comparability of utilities will fully overrule this gain.

As long as there is no overall consensus on the use of utilities and how to measure them, it will remain a real challenge to deal with this issue properly. Therefore, the use of utilities derived from clinically relevant outcome measures for cost-utility analyses is warranted only under strict conditions. Because of the difference in scope of both methods, utility values derived from clinical outcome measures are not comparable with those derived from a generic preference-based QoL instrument, even though they appear to lie on the same scale (0 to 1). The development of a utility-function bridging the gap between clinically relevant outcome measures and utilities is valuable for research purposes, although there is concern about the application of our results in economic evaluations. First of all, when health states in a preference elicitation study solely consist of clinical outcome measures, rather than general items of QoL, focusing effects may occur. ${ }^{16,17}$ The context of the valuation exercise is namely more specific, and therefore an exaggeration of health problems may occur, which will be reflected by lower utilities. Secondly, and in contrast to the previous statement, an upward bias of utility scores may occur, as the focus of clinical measures is solely on the physiological aspects of the disease itself, therefore, capturing co-morbidities and/or psychological aspects of the disease might be difficult. ${ }^{16,17}$ Thirdly, developing a utility-function from clinical outcome measures to utilities is not a clear-cut exercise. Decisions made may vary per study and will, therefore, hamper comparability between studies. $^{18,19}$

\section{Conclusions}

This study provides a utility-function for epilepsy health states and gives insight into the relative impact of clinical outcomes in epilepsy on QoL. The possible increase in sensitivity 
of outcome measures by using our utility-function comes at the price of a loss of comparability with other utilities. We, therefore, advise only using these utility values (or others) in economic evaluations when quality of life is not directly measured in a study and hence, no health state utilities are available, or when there is convincing empirical evidence of the insensitivity of a generic QoL-instrument within the field of epilepsy. Furthermore, when doing so, explicitly state the imposed bias, as using these diseasespecific utilities may come at the cost of comparability with other utility values within and across medical conditions and diseases. Finally, it is worth stating that all forms of translation-methods are always 'second best', and the existence of these techniques should not be taken as an argument to rely on them instead of obtaining utilities via generic preference-based QoL measurements in prospectively designed studies.

\section{Acknowledgements}

This paper was discussed during the LowLands Health Economists' Study Group (LoLaHESG). We would like to thank Dr. Margreet Franken for her valuable comments on the previous version of this paper. This study was funded by the Netherlands Organization for Health Research and Development (ZonMw), grant application number 171002204 and by the HTAcademy scholarship (2011) from Pfizer. 


\section{References}

1. Ramsey S, et al. Good research practices for cost-effectiveness analysis alongside clinical trials: the ISPOR RCT-CEA task force report. Value Health 2005;8(5):52 1-533.

2. O'donoghue M, Duncan J, Sander J. The national hospital seizure severity scale: a further development of the Chalfont seizure severity scale. Epilepsia 1996;37(6):563-571.

3. Drummond $M$, et al. Methods for the economic evaluation of health care programmes: Oxford University Press. New York, 2005.

4. Messori A, et al. Adjunctive lamotrigine therapy in patients with refractory seizures: a lifetime cost-utility analysis. Eur J Clin Pharmacol 1998;53(6):421-427.

5. Forbes RB, et al. Cost-utility analysis of vagus nerve stimulators for adults with medically refractory epilepsy. Seizure 2003;12(5):249-256.

6. Carroll AE, Downs SM. Improving decision analyses: parent preferences (utility values) for pediatric health outcomes. J Pediatr 2009; 155(1):21-25. e5.

7. Kang $\mathrm{H}-\mathrm{J}$, et al. The utility score of epilepsy with partial seizure measured by TTO, VAS, and EQ-5D in the general Korean population. Epilepsy Res 2014;108(5):963-971

8. McDonough CM, Tosteson AN. Measuring preferences for cost-utility analysis. Pharmacoeconomics 2007;25(2):93-106.

9. De Wit GA, Busschbach JJ, De Charro FT. Sensitivity and perspective in the valuation of health status: whose values count? Health Econ 2000;9(2):109-126.

10. Lamers $L M$, et al. The Dutch tariff: results and arguments for an effective design for national EQ-5D valuation studies. Health Econ 2006;15(10):1121-1132.

11. Group TE. EuroQol-a new facility for the measurement of health-related quality of life. Health Policy 1990; 16(3):199-208.

12. Verbeke G. Linear mixed models for longitudinal data, in Linear mixed models in practice. 1997, Springer. 63-153.

13. Robinson A, Spencer A. Exploring challenges to TTO utilities: valuing states worse than dead. Health Econ 2006; 15(4):393-402.

14. Dolan $P$, et al. The time trade-off method: Results from a general population study. Health Econ 1996;5(2):141-154.

15. Dolan $P$. The measurement of health-related quality of life for use in resource allocation decisions in health care. Handbook of health economics 2000; 1:1723-1760.

16. Brazier J, Tsuchiya A. Preference-based condition-specific measures of health: what happens to cross programme comparability? Health Econ 2010;19(2):125-129.

17. Fryback DG, Lawrence WF. Dollars may not buy as many QALYs as we think: a problem with defining quality-of-life adjustments. Med Decis Making 1997; 17(3):276-284.

18. Arnesen T, Trommald M. Are QALYs based on time trade-off comparable?-A systematic review of TTO methodologies. Health Econ 2005; 14(1):39-53.

19. Lenert LA, et al. The effect of search procedures on utility elicitations. Med Decis Making 1998;18(1): 76-83.

20. Bernert $S$, et al. Comparison of Different Valuation Methods for Population Health Status Measured by the EQ-5D in Three European Countries. Value Health 2009; 12(5):750-758.

21. CBS. Population numbers. 2013; Available from: http://statline.cbs.nl/StatWeb/publication/?VW= $T \& D M=S L E N \& P A=7461$ eng \&LA $=E N$. 



\section{Mapping of the QOLIE-3 1P to EQ-5D-5L utilities and comparison of the responsiveness of both instruments in epilepsy}
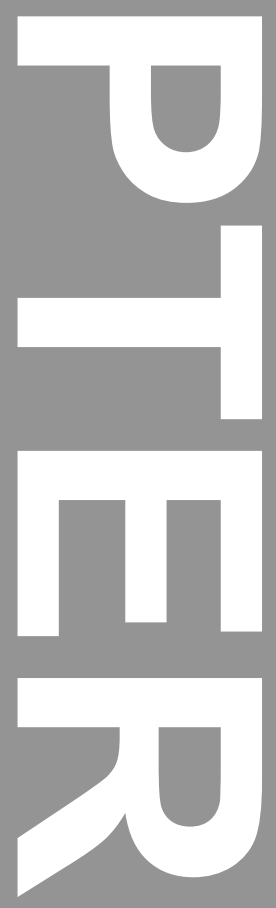

B.F.M. Wijnen

I. Mosweu

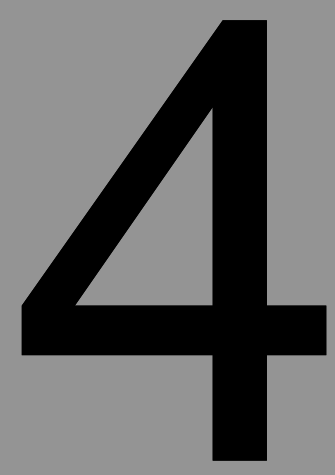
M.H.J.M. Majoie

L. Ridsdale R.J.A. de Kinderen S.M.A.A. Evers P. McCrone 


\section{Abstract}

\section{Objective}

To investigate the responsiveness of and correlation between the EQ-5D-5L and the QOLIE-31P in patients with epilepsy, and develop a mapping function to predict EQ-5D$5 \mathrm{~L}$ values based on the QOLIE-31P for use in economic evaluations.

\section{Methods}

The dataset was derived from two clinical trials, the ZMILE study in the Netherlands and the SMILE study in the UK. In both studies, patients' quality of life using the EQ-5D-5L and QOLIE$31 \mathrm{P}$ was measured at baseline and 12 months follow-up. Spearman's correlations, effect sizes (EF) and standardized response means (SRM) were calculated for both the EQ-5D-5L and QOLIE-31P domains and sub scores. Mapping functions were derived using ordinary least square (OLS) and censored least absolute deviations models.

\section{Results}

A total of 509 patients were included in this study. Moderately strong significant correlations were found between both instruments. The EQ$5 \mathrm{D}-5 \mathrm{~L}$ showed high ceiling effects and small EFs and SRMs, whereas the QOLIE-31P did not show ceiling effects and also showed small to moderate EFs and SRMs. Results of the different mapping functions indicate that the highest adjusted R2 we were able to regress was 0.265 using an OLS model with squared terms, leading to a mean absolute error of 0.103 .

\section{Conclusion}

Results presented in this study emphasize the importance of the development of conditionspecific preference-based instruments which can be used within the QALY framework, and hence incorporated as an important supplement in economic evaluations. Development of such instruments may ensure that the benefits of health-care interventions are more adequately reflected in QALY estimates. 


\section{Introduction}

Epilepsy is a disorder of the brain, characterized by recurrent seizures. Seizure episodes are a result of excessive electrical discharges in a group of brain cells. Different parts of the brain can be the site of such discharges. These discharges result in a variety of clinical manifestations, depending on where they occur in the brain. The clinical manifestations can vary from the briefest lapses of attention or muscle jerks to severe and prolonged convulsions. ${ }^{1}$

In economic evaluation, both in general and in the field of epilepsy, the quality adjusted life year (QALY) is routinely used as a summary measure of health outcome for economic evaluations, which incorporates the impact on both the quantity and quality of life (QoL). For example, the use of QALYs is required by the National Institute for Health and Clinical Excellence (NICE) in England and Wales ${ }^{2}$ and the Healthcare institute in the Netherlands ${ }^{3}$ for an intervention to be reimbursed. The utility part of QALYs requires health state values as QALYs are calculated based on the time spent in a specific health state multiplied by the corresponding utility of that health state. Commonly used measures to include in the QALYs are generic utility measures, such as the EuroQol 5 dimensions 5 levels (EQ-5D-5L), 4,5 Short Form 6 dimensions (SF-6D) ${ }^{6}$ and the Health Utility Index (HUI). ${ }^{7}$ Generic utility instruments are designed to be applicable in a large variety of conditions.

However, there are instances, especially in clinical research were some generic utility measures fail to capture changes that, even if small, are important to patients. Some studies use condition-specific or condition-specific utility measures to address this limitation. It is suggested that these instruments are likely to be more responsive than generic instruments, whose strengths include breadth and applicability across conditions and interventions. ${ }^{8}$ The responsiveness of an instrument is likely to be dependent on several factors such as the nature of the condition and the domains included in the instrument. For example, the EuroQOI-5D-3L (i.e. a generic utility instrument) has been demonstrated to correlate moderately to good with criterion measures in patients with chronic low back pain. ${ }^{9}$ In contrast, the EQ-5D-3L was deemed unsuitable for people with dementia, leading to the development of a condition-specific questionnaire (DEMQOL). ${ }^{10}$ In epilepsy, the EQ-5D$3 \mathrm{~L}$ has been shown to correlate well with another generic quality of life instrument, the 15D-instrument. ${ }^{11}$ However, in patients with newly diagnosed focal epilepsy, the EuroQol5D-3L was compared to an epilepsy-specific instrument (NEWQOL-6D) and was shown to be less responsive than the NEWQOL-6D. ${ }^{12}$ Selai et al. ${ }^{13}$ examined the use of the EQ$5 \mathrm{D}-3 \mathrm{~L}$ in people with epilepsy and concluded that adaptation, seizures, and the stigma of epilepsy considerably impair quality of life but are not captured using the EQ-5D-3L, which limit its applicability. ${ }^{13}$

Wiebe et al. ${ }^{8}$ evaluated 43 randomized controlled trials which used generic and specific QoL instruments and concluded that specific instruments are more responsive than generic tools. Furthermore, they stress that investigators may come to misleading conclusions by using generic instruments. However, condition-specific measures lack cross program comparability. Furthermore, if a condition-specific quality of life instrument were used for 
the calculation of QALYs, the valuation set should be constructed according to the same principles as generic utility measures (i.e. the multi attribute utility theory ${ }^{14}$ ), which is often not the case. An alternative option would be to derive well-conducted and validated mapping functions to map condition-specific outcomes to generic utilities. A mapping function is a regression equation used to predict values of, in this case, a generic utility instrument, using scores/values from a condition-specific instrument as regressors (also known as 'cross-walking'). ${ }^{15}$ Albeit not resolving issues regarding insensitivity of generic instruments, mapping is a solution which enables health state utilities to be predicted when no preference-based measure has been included in the study. ${ }^{15,16}$ Such mapping functions are supposed to yield utility values comparable generic instruments. ${ }^{16}$ However, the performance of a mapping function is dependent on and requires a degree of overlap between both measures and that the two measures are administered on the same population $^{15,17}$ The aim of this study is to compare the EQ-5D-5L and an often used condition-specific QoL instrument, the Quality of Life in Epilepsy-Patients-Weighted $31 \mathrm{p}$ (QOLIE-31P). ${ }^{18}$ The objective of this study is to investigate the responsiveness of and correlation between the EQ-5D-5L and the QOLIE-31P in patients with epilepsy. In addition, we aim to develop a mapping function to predict EQ-5D-5L values based on the QOLIE-31P for use in economic evaluations.

\section{Methods}

\section{QOLIE-31P}

The QOLIE-3 1-P is a condition-specific QoL instrument which consists of 38 items assessing 7 domains of epilepsy: seizure worry, overall QOL, emotional well-being, energy-fatigue, cognitive functioning including memory, medication effects, social functioning and an overall score. In addition, for each domain, questions regarding how much distress a person feels about problems and worries related to epilepsy are included. Each domain is scored on a scale ranging from 0 to 100 . Afterwards a final score can be calculated using weights derived from the amount of distress related to each domain. The final score ranges from 0 to 100, in which higher values indicate a better QoL. ${ }^{19}$

\section{$E Q-5 D-5 L$}

The EQ-5D-5L is a generic QoL instrument which consists of five dimensions: mobility, selfcare, usual activities, pain/discomfort, anxiety/depression, each of which can have one of five responses ${ }^{4,5}$ (e.g. no pain, slight pain, moderate pain, severe pain and extreme pain). This measure produces a possible 3125 distinct health states ranging from 11111 (full health) to 55555 (worst). The EQ-5D-5L was valued using both the Dutch and the UK tariffs. $^{5,20,21}$ 


\section{Data set}

The SMILE study data ${ }^{22,23}$ and the ZMILE study data ${ }^{24}$ were used for the analyses. Both studies examined the (cost-) effectiveness of a self-management program for patients with epilepsy. Follow-up data was available for 12 months in both studies. Inclusion criteria for both studies were similar. However, the SMILE study included patients from age $\geq 16$ years whereas the ZMILE study included patients aged $\geq 18$ years. Patients with complete data for each of the measures across each time point were included.

For the direct response mapping, the data set was randomly split (using the "approximately $50 \%$ of the cases"-function in SPSS) into two separate data sets: 1) the "estimation sample" ( $N=283)$, which was used to derive the mapping functions; 2) the "validation sample" ( $\mathrm{N}=224)$ which was used to validate the mapping functions.

\section{Responsiveness analyses}

Descriptive analyses are presented for patient characteristics. To measure concurrent validity (i.e. the strength of the relationship between measures of the same concept) Spearman's correlation was calculated between the domains and total scores of the EQ5D-5L and the QOLIE-3 1P. Spearman's correlation was used due to the skewed nature of the data, especially EQ-5D-5L values. Strong correlations indicate that the preferencebased measures are assessing related constructs. Correlations are considered weak if scores are less than 0.3 , moderate if scores are between 0.3 and less than 0.7 , and strong if scores are 0.7 or higher. ${ }^{25}$ Bonferroni correction was applied to account the p-values for multiple testing. $^{26}$

To determine the predictive validity or responsiveness of both instruments (i.e. the ability of an instrument to detect relevant changes in QoL over time) the Standardized Response Mean (SRM) and effect size (EF) were calculated. The SRM is a standard indicator of change across measures and time points and was calculated by $S R M=(M 1-M 2) /(S D 1-$ SD2), whereas $M 1$ is the mean pre-assessment and the $M 2$ is the mean post assessment and SD1 and SD2 are the standard deviations of both assessments. ${ }^{25}$ SRMs of less than 0.2 are considered small, 0.5 moderate, and 0.8 large. $^{25,27}$ The EF is calculated as the difference between follow-up and baseline divided by the standard deviation of the group's baseline scores. The SRM and EF were calculated for those patients amongst whom a change in health state was observed between baseline and follow-up. Floor and ceiling effects were examined. For each questionnaire the proportion of respondents with a minimum score (referred to as 'floor effects') or a maximum score (referred to as 'ceiling effects') was calculated. If a large proportion of the population is at the floor (lowest possible score) or ceiling (highest possible score), then this impairs the ability of the measure to pick up decreases or increases in QoL, respectively. ${ }^{12}$ The ES and the SRM are the most common measures for responsiveness. Positive values reflect (standardized) improvements in the number of standard deviations of the baseline scores (ES) or the score differences (SRM) (i.e. unit-free). ${ }^{28}$ 


\section{Mapping approach}

To estimate EQ-5D-5L utility scores based on the QOLIE-31P, direct response mapping was used to regress QOLIE-31P scores to EQ-5D-5L scores. In direct mapping, a regression equation is used to predict the values of the EQ-5D-5L using scores/values from the QOLIE-3 1P as regressors. Next, the coefficients of the model are used to carry out the conversion from the source measure to the target measure in the required dataset. ${ }^{29}$

Spearman's correlations of the independent variables were used to determine whether there was collinearity between independent variables, which would then be removed from the analyses. A collinearity threshold of $>0.70$ was used. $^{30}$

Ordinary least-squares (OLS) and Censored least absolute deviations (CLAD) regression was used as to estimate the model. The OLS is the most commonly used model in mapping studies. ${ }^{15,31}$ However, it is unable to restrict for the range of values and may lead to implausible predicted values (e.g. EQ-5D values above 1). The CLAD model was therefore used as it has the ability to account for censored or bounded data. In addition, it is robust to heteroscedasticity and can also be used for skewed data. ${ }^{15,32}$

As the aim of this study was to derive a predictive model, all items (domains) were included in the model despite their significance level, which is often considered best practice. $^{33-35}$

Furthermore, no attempt was made to predict the individual EQ-5D-5L dimensions separately as this has been shown to be a less efficient strategy or to give similar results in terms of prediction. ${ }^{36}$

For both the OLS and the CLAD model, specifications of the mapping functions were constructed as proposed by Brazier et al. (2010). ${ }^{15}$ We started with a simple additive model by predicting EQ-5D values from the total QOLIE-31P scores including age, gender, employment, and living arrangements (model 1). Next, the EQ-5D-5L values were predicted from the 7 QOLIE-31P dimension sub scores (model 2). To relax the assumptions of the simple additive model, squared terms for dimension sub scores were included in the model (model 3). ${ }^{15}$ As suggested by Brazier et al. (2010) only significant squared terms were included in the model to reduce the number of variables. ${ }^{15}$

The predictive validity of the mapping models was assessed by using: 1) the goodness of fit as assessed using adjusted/pseudo R-squared (OLS and CLAD) in the estimation sample; and 2) the predictive performance of the models in the validation sample was assessed using the mean absolute error (MAE).

All analyses were done in STATA 15 (StataCorp, College Station, TX, USA).

\section{Results}

The dataset consisted of 509 patients of which 102 patients were recruited for the ZMILE study and 407 for the SMILE study. In total, $53.0 \%$ of the patients were female and the majority of the patients were aged between 25 and 44 years old. Most of the patients had a household or lived with others $(73.1 \%)$ and $51.3 \%$ of the patients were unemployed. 
Mean quality of life according to the EQ-5D-5L was 0.86 and mean condition-specific quality of life according to the QOLIE-31P was 65.82. More detail regarding the characteristics of the population(s) is reported in Table 4.1.

Table 4.1 (Baseline) characteristics of the population

\begin{tabular}{|c|c|c|c|}
\hline Characteristics & $\begin{array}{l}\text { ZMILE sample } \\
\qquad(n=102)\end{array}$ & $\begin{array}{l}\text { SMILE sample } \\
\quad(n=407)\end{array}$ & $\begin{array}{c}\text { Total } \\
(n=509)\end{array}$ \\
\hline \multicolumn{4}{|l|}{ Gender } \\
\hline Male & $50(49.0 \%)$ & $185(45.5 \%)$ & $235(46.2 \%)$ \\
\hline Female & $52(51.0 \%)$ & $219(53.8 \%)$ & $271(53.2 \%)$ \\
\hline Missing values & 0 & 3 & \\
\hline \multicolumn{4}{|l|}{ Age in years } \\
\hline $16-24$ & $17(16.7 \%)$ & $46(11.3 \%)$ & $63(12.4 \%)$ \\
\hline $25-44$ & $42(41.2 \%)$ & $194(47.7 \%)$ & $236(46.4 \%)$ \\
\hline $45-64$ & $37(36.2 \%)$ & $142(34.9 \%)$ & $179(35.2 \%)$ \\
\hline \multirow[t]{2}{*}{$\geq 65$} & $6(5.9 \%)$ & $22(5.4 \%)$ & $28(5.5 \%)$ \\
\hline & 0 & 3 & \\
\hline \multicolumn{4}{|l|}{ Living arrangements, $\mathrm{n}(\%)$} \\
\hline Household / living with others & $67(65.7 \%)$ & $305(74.9 \%)$ & $372(73.1 \%)$ \\
\hline Living alone & $26(25.5 \%)$ & $95(23.4 \%)$ & $121(23.8 \%)$ \\
\hline Other arrangements & $4(3.9 \%)$ & $4(1 \%)$ & $8(1.6 \%)$ \\
\hline Missing values & 5 & 3 & \\
\hline \multicolumn{4}{|l|}{ Employment } \\
\hline Not unemployed & $54(52.9 \%)$ & $207(50.9 \%)$ & $261(51.3 \%)$ \\
\hline Specifically employed or student & $44(43.1 \%)$ & $197(48.4 \%)$ & $241(47.4 \%)$ \\
\hline Missing values & 4 & 3 & \\
\hline \multicolumn{4}{|l|}{ Quality of life } \\
\hline EQ-5D-5L baseline & 0.83 & 0.87 & 0.86 \\
\hline EQ VAS & 74.77 & 67.00 & 68.53 \\
\hline QOLIE-3 1 P baseline & 64.74 & 66.05 & 65.82 \\
\hline
\end{tabular}

\section{Validity \& responsiveness}

An assessment of the strength of the relationship between the EQ-5D-5L and the QOLIE$31 \mathrm{P}$ based on the Spearman's correlation coefficient, showed moderately strong significant correlations between both instruments for the total score (Table 4.2). Only a few statistically significant correlations were found between the sub scores of the QOLIE-3 1P and the sub scores of the EQ-5D-5L. All subscores of the QOLIE-31P did significantly correlate with the total EQ-5D-5L scores. At baseline and 12 months follow-up ceiling effects on the EQ-5D-5L were substantial with $37.8 \%$ and $33.5 \%$ of the patients reporting the maximum score. No ceiling effects were found for the QOLIE-3 1 P.

Details regarding the EF and SRM are presented in Table 4.3. EFs and SRMs, all appear to be relatively small. Both the EF and SRM estimates are smaller for the EQ-5D-5L than the QOLIE-3 1P. For the EQ-5D-5L, values range from -0.017 to 0.043 for the EF and from -0.023 to 0.025 for the SRM which would be considered small. The EF and SRM values for the QOLIE-31P range from 0.082 to 0.290 (EF) and from 0.07 to 0.270 (SRM), which would be regarded as small to moderate. 
Table 4.2 Correlation coefficients between QOLIE-31P values to EQ-5D-5L values.

\begin{tabular}{lcccccc}
\hline & $\begin{array}{c}\text { EQ-5D } \\
\text { mobility }\end{array}$ & $\begin{array}{c}\text { EQ-5D self- } \\
\text { care }\end{array}$ & $\begin{array}{c}\text { EQ-5D } \\
\text { usual } \\
\text { activities }\end{array}$ & $\begin{array}{c}\text { EQ-5D } \\
\text { pain }\end{array}$ & $\begin{array}{c}\text { EQ-5D } \\
\text { Anxiety and } \\
\text { depression }\end{array}$ & $\begin{array}{c}\text { Total } \\
\text { EQ-5D } \\
\text { score }\end{array}$ \\
\hline QOLIE-3 1P Energy & -0.2330 & -0.1896 & -0.1991 & $-0.2689^{*}$ & -0.2293 & $0.4499^{*}$ \\
QOLIE-3 1P Mood & -0.1252 & -0.0285 & -0.1725 & -0.1743 & $-0.3823^{*}$ & $0.4881^{*}$ \\
QOLIE-3 1P Daily activities & -0.2350 & -0.2138 & $-0.2557^{*}$ & $-0.2644^{*}$ & $-0.2422^{*}$ & $0.4335^{*}$ \\
QOLIE-3 1P Cognition & -0.1041 & -0.0607 & -0.1906 & -0.1661 & $-0.2539^{*}$ & $0.2948^{*}$ \\
QOLIE-3 1P Medication effects & -0.0569 & -0.1061 & -0.1744 & -0.1969 & -0.2304 & $0.2730^{*}$ \\
QOLIE-3 1P Seizure worry & -0.1099 & -0.0802 & -0.1502 & -0.1901 & $-0.3057^{*}$ & $0.3906^{*}$ \\
QOLIE-3 1P Overall QOL & -0.1794 & -0.1620 & -0.2245 & -0.1808 & $-0.3142^{*}$ & $0.4049^{*}$ \\
Total QOLIE-31P score & -0.2000 & -0.1476 & $-0.2855^{*}$ & $-0.3171^{*}$ & $-0.3354^{*}$ & $0.5653^{*}$ \\
\hline
\end{tabular}

* Significant correlation at $5 \%$ level.

Table 4.3 Standardized response means for QOLIE-31P and EQ-5D-5L.

\begin{tabular}{lccc}
\hline & $\begin{array}{c}\text { Mean difference between } \\
\text { BS and FU 1 2M }\end{array}$ & Effect size & $\begin{array}{c}\text { Standardized response } \\
\text { mean }\end{array}$ \\
\hline Total EQ-5D-5L score & -0.004 & -0.017 & -0.023 \\
EQ-5D mobility & 0.276 & 0.010 & 0.003 \\
EQ-5D self-care & 0.305 & 0.010 & 0.003 \\
EQ-5D usual activities & 2.600 & 0.043 & 0.025 \\
EQ-5D pain & 0.619 & 0.012 & 0.006 \\
EQ-5D anxiety & -1.847 & -0.017 & -0.019 \\
Total QOLIE-3 1P score & 2.414 & 0.187 & 0.212 \\
QOLIE-3 1P energy & 1.875 & 0.110 & 0.080 \\
QOLIE-3 1P mood & 1.906 & 0.082 & 0.070 \\
QOLIE-3 1P daily activities & 8.317 & 0.284 & 0.270 \\
QOLIE-3 1P cognition & 3.196 & 0.131 & 0.125 \\
QOLIE-3 1P medication effects & 5.878 & 0.177 & 0.178 \\
QOLIE-3 1P seizure worry & 7.696 & 0.290 & 0.254 \\
QOLIE-3 1P overall QOL & 2.963 & 0.128 & 0.116 \\
\hline
\end{tabular}

EQ-5D-5L: EuroQol 5 dimensions with 5 levels; NL: the Netherlands; UK: United Kingdom.

\section{Mapping functions}

The EQ-5D index scores had a somewhat bimodal distribution, and the QOLIE-3 IP index scores were normally distributed (see Supplemental Figure S4.1). The inclusion of age was shown to have a significant effect on the prediction of EQ-5D-5L scores. All other demographic variables were excluded from the analyses. In addition, there was a significant effect associated with country (i.e. SMILE- or ZMILE-dataset). Hence, age and country were included in all mapping function.

For the OLS mapping functions, model 3 performed best with an MAE of 0.103 and an adjusted $R^{2}$ of 0.265 . Inclusion of age significantly improved the model, hence a model without age was only constructed for OLS. For the CLAD mapping functions, CLAD model 3 performed best with a MAE of 0.097 and a pseudo $R^{2}$ of 0.160 . It should be noted, however, that including squared terms did only improve adjusted/pseudo $R^{2}$ values and only marginally improved MAE in the estimation sample (see Table 4.4). All models predicted values above 1 (full health), of which OLS model 2 was closest to 1 with 
maximum values of 1.020 . A graphical representation of the model fits is presented in Figure 4.1.

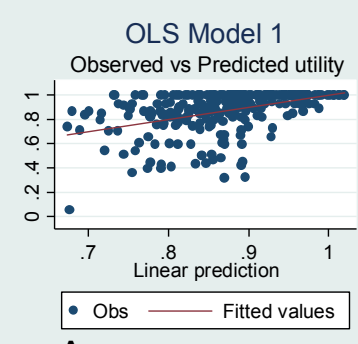

A

CLAD Model 1

Observed vs Predicted utility

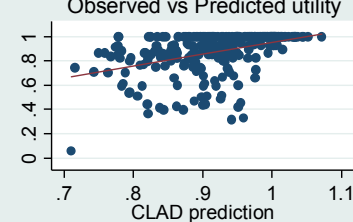

- Obs $\longrightarrow$ Fitted values

D
OLS Model 2

Observed vs Predicted utility

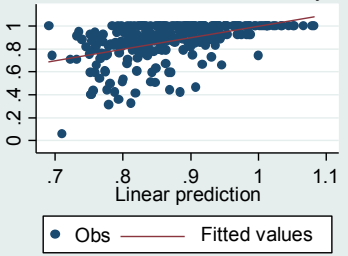

B

CLAD Model 2

Observed vs Predicted utility

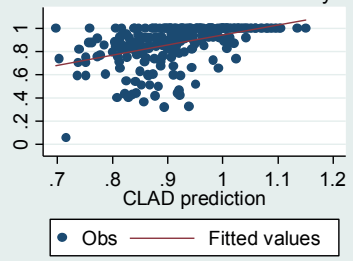

E
OLS Model 3

Observed vs Predicted utility

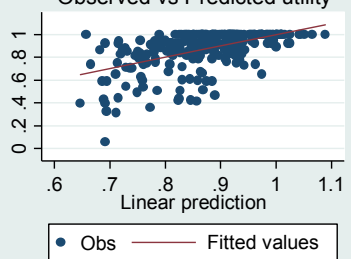

C

CLAD Model 3

Observed vs Predicted utility

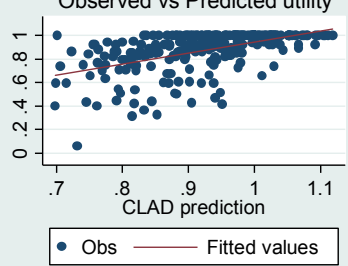

F

Figure 4.1 Overview of observed and predicted utilities for OLS and CLAD models

Table 4.4 Table 4 Summary of observed and predicted values for all models in estimation dataset (N=283).

\begin{tabular}{|c|c|c|c|c|c|}
\hline & \multirow{2}{*}{$\begin{array}{c}\text { Observed EQ- } \\
\text { 5D utility }\end{array}$} & \multicolumn{4}{|c|}{ Predicted EQ-5D utilities } \\
\hline & & $\begin{array}{c}\text { Total QOLIE-3 IP } \\
\text { scores }\end{array}$ & $\begin{array}{l}\text { Total QOLIE-3IP } \\
\& \text { age \& country }\end{array}$ & $\begin{array}{c}\text { QOLIE-3 IP } \\
\text { domain scores \& } \\
\text { age \& country }\end{array}$ & $\begin{array}{c}\text { Domain scores \& } \\
\text { squared terms \& age } \\
\& \text { country }\end{array}$ \\
\hline & & OLS Model 0 & OLS Model 1 & OLS Model 2 & OLS Model 3 \\
\hline Mean & 0.867 & 0.868 & 0.868 & 0.871 & 0.872 \\
\hline Minimum & 0.055 & 0.668 & 0.673 & 0.690 & 0.647 \\
\hline Maximum & 1.000 & 1.026 & 1.020 & 1.082 & 1.086 \\
\hline MAE & & 0.110 & 0.107 & 0.103 & 0.103 \\
\hline \multirow[t]{2}{*}{ Adjusted $R^{2}$} & & 0.151 & 0.180 & 0.211 & 0.265 \\
\hline & & & CLAD Model 1 & CLAD Model 2 & CLAD Model3 \\
\hline Mean & 0.867 & - & 0.915 & 0.916 & 0.920 \\
\hline Minimum & 0.055 & - & 0.711 & 0.697 & 0.698 \\
\hline Maximum & 1.000 & - & 1.072 & 1.150 & 1.119 \\
\hline MAE & & - & 0.099 & 0.099 & 0.097 \\
\hline Pseudo $R^{2}$ & & - & 0.116 & 0.129 & 0.160 \\
\hline
\end{tabular}

MAE: Mean Absolute Error; OLS: Ordinary Least Squares; CLAD: Censored Least Absolute Deviations; POS: Palliative Care Outcome Scale 
When assessing the MAE in the validation sample, using the mapping functions derived from the estimation sample, OLS model 1 performed best with a MAE of 0.114. Likewise, for the CLAD mapping functions, model 1 performed best with a MAE value of 0.109 (see Table 4.5). Given the higher adjusted/pseudo $R^{2}$ values of model 3 compared to model 1 for both OLS and CLAD, and give the lower MAE for OLS model 3 compared to CLAD model 3 in the validation sample, the best mapping function would be OLS model 3 . The regression coefficients for this model are presented in Supplemental Table S4.1.

Table 4.5 Summary of observed and predicted values for all models in validation dataset ( $N=224)$.

\begin{tabular}{|c|c|c|c|c|}
\hline & \multirow{2}{*}{$\begin{array}{c}\text { Observed EQ-5D } \\
\text { utility }\end{array}$} & \multicolumn{3}{|c|}{ Predicted EQ-5D utilities } \\
\hline & & $\begin{array}{c}\text { Total QOLIE-3 IP \& age } \\
\text { \& country }\end{array}$ & $\begin{array}{c}\text { QOLIE-3 IP domain } \\
\text { scores \& age \& country }\end{array}$ & $\begin{array}{c}\text { Domain scores \& } \\
\text { squared terms \& age \& } \\
\text { country }\end{array}$ \\
\hline & & OLS Model 1 & OLS Model 2 & OLS Model 3 \\
\hline Mean & 0.863 & 0.865 & 0.865 & 0.865 \\
\hline Minimum & 0.054 & 0.649 & 0.685 & 0.629 \\
\hline Maximum & 1.000 & 1.039 & 1.100 & 1.084 \\
\hline \multirow[t]{2}{*}{ MAE } & & 0.114 & 0.116 & 0.118 \\
\hline & & CLAD Model 1 & CLAD Model 2 & CLAD Model 3 \\
\hline Mean & 0.863 & 0.912 & 0.907 & 0.911 \\
\hline Minimum & 0.054 & 0.682 & 0.688 & 0.699 \\
\hline Maximum & 1.000 & 1.062 & 1.136 & 1.120 \\
\hline MAE & & 0.109 & 0.117 & 0.119 \\
\hline
\end{tabular}

\section{Discussion}

The aim of this study was to compare the responsiveness of the QOLIE-31P and the EQ5D-5L in epilepsy and to predict EQ-5D-5L values based on QOLIE-3 1P scores with the development of a mapping function. Looking at concurrent validity, although the correlations were significant, the strength was only moderate between both instruments when looking at the total score. This may imply that both instruments are only measuring the same concept to some extent and impose conceptual differences.

The EQ-5D-5L showed substantially high ceiling effects and was demonstrated to have rather small EFs and SRMs whereas the QOLIE-3 1 P did not show ceiling effects and had small to moderate EFs and SRMs. This indicates that the QOLIE-31P has an overall higher responsiveness based on EF, SRM and ceiling effects.

Furthermore, this study provides a mapping function which can be used in (future) economic evaluations to map QOLIE-3 1 P data to EQ-5D-5L values.

The relatively small EFs and SRMs may be explained by a lack of responsiveness of both instruments, especially the EQ-5D-5L. However, part of these small estimates can be explained by the fact that the patients' health state did not change much over time. The EF and SRM were calculated in all patients who had any change during follow-up, due to the 
lack of a known clinically meaningful difference for both instruments, this led to an underestimation of the EF and SRM. The EQ-5D-5L, however, performed substantially worse than the QOLIE-31P.

Using different mapping functions, the highest adjusted $R^{2}$ we were able to regress was 0.265 using an OLS model with squared terms, which led to a MAE of 0.103 . Overall, this model performed best given the results within the estimation and validation sample. Although theoretically preferred, the use of a CLAD model did not perform better than the OLS model, especially in the validation sample. Mixed results have been reported in studies using CLAD models, ${ }^{17}$ with some concluding that CLAD improved the model fit ${ }^{37,38}$ and others concluding that the improvement of CLAD over OLS was small or did not have an impact. ${ }^{39}$ The adjusted/pseudo $\mathrm{R}^{2}$ values found in this study were relatively small, which is not uncommon. In a review of Brazier et al. (2010), it was found that models mapping a generic instrument onto a generic preference-based measure achieved an adjusted $R^{2}$ of more than 0.5 within sample. However, in studies examining the fit of functions mapping from condition-specific to generic measures, results were more variable ranging from 0.17 to $0.51 .{ }^{15}$ In addition, errors were often larger for models mapping a generic measure onto a generic preference-based measure. ${ }^{15,17}$ Likewise, the usefulness of our mapping function in economic evaluations is questionable given the relatively large mean absolute error and poor model fit.

Another way of mapping would be to use a model to predict responses of each of the five dimensions of the EQ-5D-5L from the QOLIE-31P (sub) scores, so called indirect response mapping or response mapping models. ${ }^{40}$ As the purpose of the mapping part of this study was to derive a regression function, this method was not applied. In addition, as mentioned above, indirect response mapping has been shown to be a less efficient strategy or to give similar results in terms of prediction. ${ }^{36}$

The use of mapping to derive EQ-5D-5L values is fundamentally limited by the degree of overlap between two instruments. ${ }^{17}$ Although several studies reported limitations with generic preference-based quality of life instruments regarding their responsiveness and ability to discriminate between health states (e.g. McTaggart-Cowan et al. ${ }^{41}$ ), the use of generic preference-based instruments is mandatory in most national guidelines for pharmaco-economic evaluations, for example in the UK and the Netherlands. ${ }^{3,42}$ However, given the limited responsiveness, low correlations, and the poor model fit of the mapping functions it may be argued that there is a need for the development of conditionspecific preference-based measures. General (non-preference based) condition-specific instruments, such as the QOLIE-31P, are an important source of evidence, however, their use in economic evaluation is severely limited because they were not designed for this purpose and, unless they are preference-based, they theoretically cannot be used to calculate quality adjusted life years (QALYs). ${ }^{14,43}$ Several attempts have been made to derive condition-specific quality of life instruments to calculate (condition-specific) QALYs, such as the development of new instruments ${ }^{44,45}$ or the development of condition-specific preference-based measures from existing instruments. ${ }^{46,47}$ Of course, such a measure could not be the sole outcome of interest for economic evaluations, as they lack the 
comparability between conditions, a distinct advantage of generic instruments. ${ }^{48} \mathrm{We}$ agree with Brazier et al. (2010), that development of a condition-specific preferencebased instrument should not be seen as an alternative to generic preference-based measures, but rather as a supplement. ${ }^{43}$ Condition-specific preference-based instruments may have an important role in ensuring that the benefits of health-care interventions are adequately reflected in QALY estimates for economic evaluations in all conditions. ${ }^{49}$

This study is subject to several limitations. First, we only investigated the use of a few mapping models, whereas a wide variety of models exists such as GLM of Tobit models. However, given the marginal differences between the models used in this study, the model fit is not likely to be improved substantially. Second, our estimations are based on a pooled data set containing data from both the UK and the Netherlands. Although inclusion criteria for both studies were similar and there was no significant difference between the countries regarding regression estimates, this may have introduced extra heterogeneity within the data. Lastly, the pooled dataset was divided in an estimation sample and a validation sample. This has the advantage that it assesses the mapping function by its prime purpose; however, it reduces the sample size of the estimation sample. The use of the whole sample for the model estimations, however, did not substantially improve the model(s).

\section{Conclusion}

There was a low to moderate correlation between the EQ-5D-5L and the QOLIE-3 1P. Both the EF and SMRs were relatively low, especially for the EQ-5D-5L. Mapping functions to regress $Q$ OLIE-3 1P values to EQ-5D-5L values did not show an optimal fit with relatively low adjusted $R^{2}$ values. The results presented in this study may emphasize the importance of the development of condition-specific preference-based instruments which can be used within the QALY framework and hence incorporated as an important supplement in economic evaluations. The development of such condition-specific preference-based quality of instruments can ensure that the benefits of health-care interventions are adequately reflected in QALY estimates for economic evaluations not only in epilepsy but for all conditions.

\section{Acknowledgements}

This study was funded by the Netherlands Organization for Health Research and Development (ZonMw), grant application number 836011018 . Data collection in the SMILE trial was funded by the National Institute for Health Research [Health Technology Assessment (HTA) programme] (project number 09/165/01). The views and opinions expressed are those of the authors and do not necessarily reflect those of the HTA programme, the UK NIHR, NHS or the Department of Health.

We would like to thank Dr. M. Dzingina (King's College London) for his help with the analyses of the results. 


\section{References}

1. World Health Organisation. Epilepsy. 2016; Available from:

http://www.who.int/mediacentre/factsheets/fs999/en/.

2. Whitehead SJ, Ali S. Health outcomes in economic evaluation: the QALY and utilities. Br Med Bull 2010;96(1):5-21.

3. Zorginstituut Nederland. Richtlijn voor het vitvoeren van economische evaluaties in de gezondheidszorg Diemen: Zorginstituut Nederland, 2015.

4. Brooks R. EuroQol: the current state of play. Health Policy 1996;37(1):53-72.

5. Devlin N, et al. Valuing health-related quality of life: An EQ-5D-5L value set for England. 2016.

6. Brazier J, Roberts J, Deverill M. The estimation of a preference-based measure of health from the SF-36.J Health Econ 2002;21(2):271-292.

7. Horsman J, et al. The Health Utilities Index (HUI(囚)): concepts, measurement properties and applications. Health Qual Life Outcomes 2003;1:54.

8. Wiebe $\mathrm{S}$, et al. Comparative responsiveness of generic and specific quality-of-life instruments. J Clin Epidemiol 2003;56(1):52-60.

9. Soer $R$, et al. Clinimetric properties of the EuroQol-5D in patients with chronic low back pain. Spine $J$ 2012;12(11):1035-9.

10. Smith SC, et al. Measurement of health-related quality of life for people with dementia: development of a new instrument (DEMQOL) and an evaluation of current methodology. Health Technol Assess 2005; 9(10): 1-93, iii-iv.

11. Stavem K, Biørnæs H, Lossius MI. Properties of the $15 \mathrm{D}$ and EQ-5D utility measures in a community sample of people with epilepsy. Epilepsy Res 2001;44(2-3):179-189.

12. Mulhern B, et al. Comparing generic and condition-specific preference-based measures in epilepsy: EQ5D-3L and NEWQOL-6D. Value in Health, 2016.

13. Selai CE, Elstner K, Trimble MR. Quality of life pre and post epilepsy surgery. Epilepsy Res 2000;38(1): 67-74.

14. Von Neumann, J. Morgenstern O. Theory of games and economic behavior. 2007: Princeton university press.

15. Brazier JE, et al. A review of studies mapping (or cross walking) non-preference based measures of health to generic preference-based measures. Eur J Health Econ 2010;1 1(2):215-225.

16. Versteegh $M M$, et al. Condition-specific preference-based measures: benefit or burden? Value Health 2012;15(3):504-513.

17. Longworth L, Rowen D. Mapping to obtain EQ-5D utility values for use in NICE health technology assessments. Value Health 2013;16(1):202-210.

18. Cramer JA, et al. Development and cross-cultural translations of a 31 -item quality of life in epilepsy inventory. Epilepsia 1998;39(1):81-88.

19. Cramer JA, Van Hammee G. Maintenance of improvement in health-related quality of life during long-term treatment with levetiracetam. Epilepsy Behav 2003;4(2):1 18-123.

20. Versteegh M, et al. Dutch Tariff for the Five-Level Version of EQ-5D. Value Health 2016;19(4):343-352.

21. Feng Y, et al. New Methods for Modelling EQ-5D-5L Value Sets: An Application to English Data. 2016, Office of Health Economics Research.

22. Krali-Hans I, et al. Self-Management education for adults with poorly controlled epILEpsy (SMILE (UK)): a randomised controlled trial protocol. BMC Neurol 2014;14:69.

23. Magill $\mathrm{N}$, et al. Self-management education for adults with poorly controlled epilepsy (SMILE (UK)): statistical, economic and qualitative analysis plan for a randomised controlled trial. Trials 2015;16:269.

24. Leenen LA, et al. (Cost)-effectiveness of a multi-component intervention for adults with epilepsy: study protocol of a Dutch randomized controlled trial (ZMILE study). BMC Neurol 2014; 14:255.

25. Cohen J. Statistical power analysis for the behavioral sciences. 1988, Hillsdale, N.J.: L. Erlbaum Associates.

26. Rosner B. Multiple comparisons: Bonferroni approach. Fundamentals of biostatistics, 2000. 5.

27. Kazis LE, Anderson JJ, Meenan RF. Effect sizes for interpreting changes in health status. Medical Care 1989;27(3):S178-S189.

28. Angst $F$, et al. Responsiveness of five condition-specific and generic outcome assessment instruments for chronic pain. BMC Med Res Methodology 2008;8(1):26. 
29. Khan KA, et al. Mapping EQ-5D Utility Scores from the PedsQL ${ }^{\top M}$ Generic Core Scales. Pharmacoeconomics 2014;32(7):693-706.

30. Tabachnick BG, Fidell LS, Osterlind SJ, Using multivariate statistics. 2001.

31. Longworth $L$, et al. Use of generic and condition-specific measures of health-related quality of life in NICE decision-making: a systematic review, statistical modelling and survey. Health Technol Assess 2014; 18(9): $1-224$.

32. Teckle $P$, et al. Mapping the FACT-G cancer-specific quality of life instrument to the EQ-5D and SF-6D. Health Qual Life Outcomes 2013;11(1):203.

33. Wu S, Harris TJ, McAuley KB, The Use of Simplified or Misspecified Models: Linear Case. The Canadian Journal of Chemical Engineering 2007;85(4):386-398.

34. Shmueli G. To explain or to predict? Statistical science 2010:289-310.

35. Greenberg E, Parks RP. A predictive approach to model selection and multicollinearity. Journal of Applied Econometrics 1997; 12(1):67-75.

36. Crott R, Briggs A. Mapping the QLQ-C30 quality of life cancer questionnaire to EQ-5D patient preferences. Eur J Health Econ 2010; 11 (4):427-434.

37. Sullivan PW, Ghushchyan V. Mapping the EQ-5D index from the SF-12: US general population preferences in a nationally representative sample. Med Decis Making 2006;26(4):401-409.

38. Payakachat N, et al. Predicting EQ-5D utility scores from the 25-item National Eye Institute Vision Function Questionnaire (NEI-VFQ 25) in patients with age-related macular degeneration. Qual Life Res 2009; 18(7): 801-813.

39. Kontodimopoulos N, et al. Mapping the cancer-specific EORTC QLQ-C30 to the preference-based EQ5D, SF-6D, and 15D instruments. Value Health 2009;12(8):1151-1157.

40. Dakin $\mathrm{H}$. Review of studies mapping from quality of life or clinical measures to EQ-5D: an online database. Health Qual Life Outcomes 2013;11:151-151.

41. McTaggart-Cowan HM, et al. The validity of generic and condition-specific preference-based instruments: the ability to discriminate asthma control status. Qual Life Res 2008; 17(3):453-462.

42. Williams I, et al. The use of economic evaluations in NHS decision-making: a review and empirical investigation. Health Technol Assess 2008; 12(7):iii, ix-x, 1-175.

43. Brazier J, Tsuchiya A. Preference-based condition-specific measures of health: what happens to cross programme comparability? Health Economics 2010;19(2):125-129.

44. Torrance $G$, et al. Development and initial validation of a new preference-based disease-specific healthrelated quality of life instrument for erectile function. Qual life Res 2004;13(2):349-359.

45. Revicki DA, et al. Integrating patient preferences into health outcomes assessment: the multiattribute Asthma Symptom Utility Index. Chest 1998; 1 14(4):998-1007.

46. Brazier J, et al. Estimation of a preference-based index from a condition-specific measure: the King's Health Questionnaire. Med Decis Making 2008;28(1):113-126.

47. Yang $Y$, et al. Deriving a preference-based measure for health from the AQLQ. in Health Economists Studying Group Meeting (HESG), London. 2006.

48. Donaldson C, et al. Should QALYs be programme-specific? J Health Econ 1988;7(3):239-257.

49. Brazier JE, et al. Developing and testing methods for deriving preference-based measures of health from condition-specific measures (and other patient-based measures of outcome). Health Technol Assess 2012; 16(32): $1-114$. 


\section{Supplemental material}

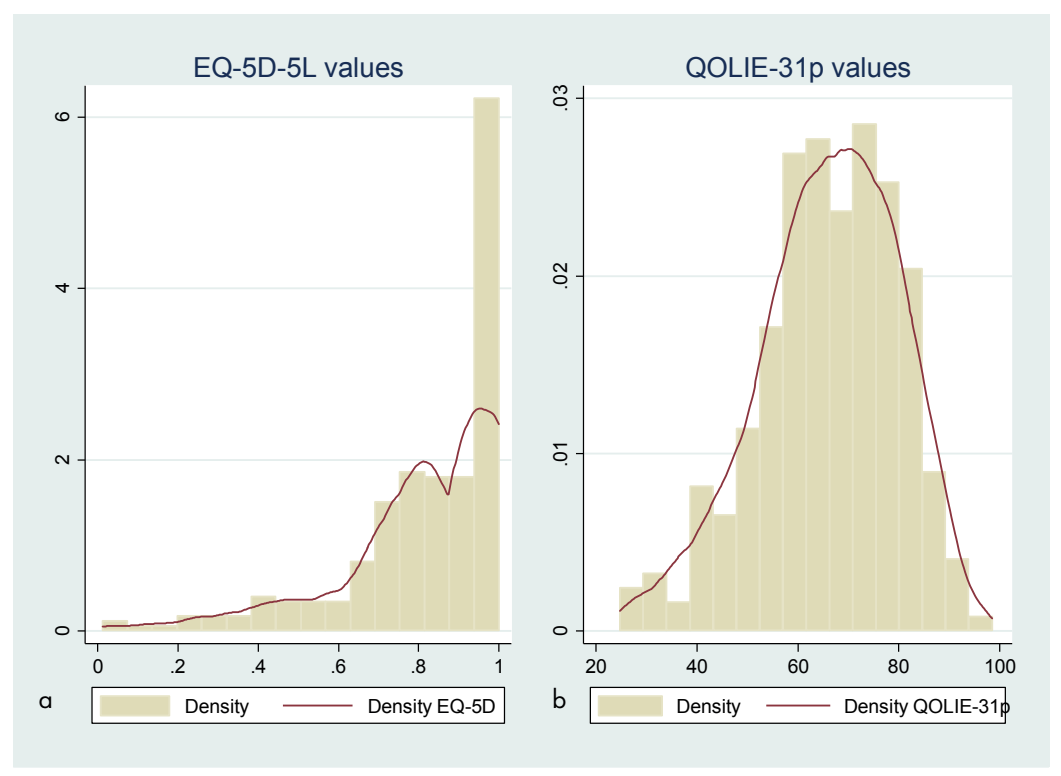

Figure S4.1 Distribution of the EQ-5D index scores (a); and distribution of the QOLIE-3 1P index scores (b)

Table S4.1 "Best" mapping function given adjusted ${ }^{R}$ values and mean absolute error rates to map QOLIE-31P values to $\mathrm{EQ}-5 \mathrm{D}-5 \mathrm{~L}$ values.

\begin{tabular}{lcc}
\hline & Coefficients & Std. Error \\
\hline (Constant) & 0.68416780 & 0.0480296 \\
Country & 0.07690210 & 0.0243357 \\
Age (years) & -0.00134930 & 0.0006669 \\
Score sub score "Seizure worry" & 0.00027910 & 0.0003756 \\
Score sub score "Overall QOL" & 0.00022530 & 0.0014603 \\
Score sub score "Emotional well-being" & 0.00431610 & 0.0014529 \\
Score sub score "Energy-fatigue" & 0.00408590 & 0.0015880 \\
Score sub score "Cognitive functioning" & -0.00009420 & 0.0003570 \\
Score sub score "Medication effects" & -0.00348630 & 0.0011060 \\
Score sub score "Social functioning" & 0.00073940 & 0.0004200 \\
Squared sub score "Energy-fatigue" & -0.00002710 & 0.0000172 \\
Squared sub score "Emotional well-being" & -0.00004190 & 0.0000143 \\
Squared sub score "Medication effects" & 0.00003180 & 0.0000102 \\
Squared sub score "Overall QOL" & 0.00000632 & 0.0000146 \\
Adjusted R & & 0.265 \\
\hline
\end{tabular}





\title{
Using best-worst scaling to
} investigate preferences in

\author{
health care:
}

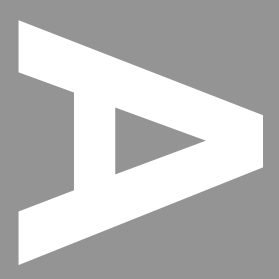

A systematic review
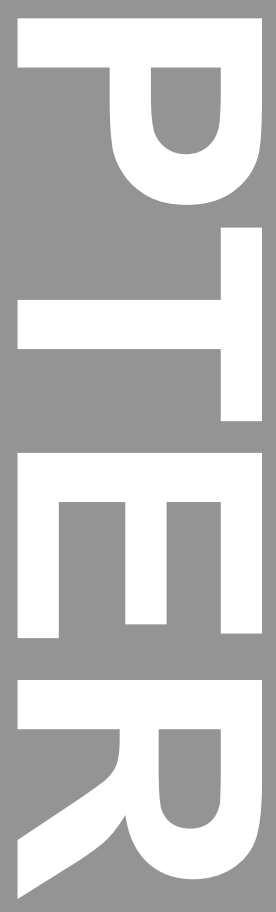

$$
\begin{array}{r}
\text { B.F.M. Wiinen }{ }^{*} \\
\text { K.L. Cheung } \\
\text { I.L.Hollin } \\
\text { A.J. Colon } \\
\text { E.M. Janssen } \\
\text { J.F. Bridges } \\
\text { S.M.A.A. Evers } \\
\text { M. Hiligsmann }
\end{array}
$$

Pharmacoeconomics 2016; 34(12), 1195-1209 * both authors contributed equally 


\section{Abstract}

\section{Introduction}

Best-worst scaling (BWS) is becoming increasingly popular to elicit preferences in health care. However, little is known about current practice and trends in the use of BWS in health care. This study aims to identify, review and critically appraise BWS in health care, and to identify trends over time in key aspects of BWS.

\section{Methods}

A systematic review was conducted, using MEDLINE (via PubMed) and EMBASE to identify all English language BWS studies available in print or online until April 2016. Two reviewers independently selected articles, systematically extracted data using a predefined extraction sheet and critically appraised the study quality using the Purpose, Respondents, Explanation, Findings, Significance (PREFS) checklist. Trends over time periods ( $\leq 2010$, 2011, 2012, 2013, 2014 and 2015) were further assessed.

\section{Results}

A total of 62 BWS were identified of which 26 were BWS object case, 29 were BWS profile case, and 7 were BWS multi-profile case. About two- thirds of studies were performed in the last two years. Decreasing sample sizes and number of factors in BWS object case, as well as the use of less complicated analytic methods was observed in recent studies. The quality of BWS studies is generally acceptable according to the PREFS checklist, except that most studies did not indicate whether the responders were similar to non-responders.

\section{Conclusion}

The use of BWS object case and BWS profile case has drastically increased in health care, especially in the last two years. In contrast with previous DCE reviews, there is an increasing use of less sophisticated analytic methods. 


\section{Background}

Investigating preferences has become popular in health care. In recent years, the need for a more patient-centred approach in health care and health technology assessment has been widely acknowledged. Hence, it is expected that eliciting preferences will become increasingly important to support health policy and clinical decisions. ${ }^{1,2}$ In order to enhance the patient-centred approach, preferences may be elicited from patients or other key players in the health sector such as clinicians and policy makers on how they value various aspects of health interventions or when designing and evaluating health care programs. ${ }^{3}$ By eliciting preferences of other key players in health sector, one may identify potential discrepancies between the stakeholders, which may be addressed to enhance patient-centred health care. ${ }^{4}$ Preference studies can provide relevant information to support health technology assessment and decisions about priorities in health care. ${ }^{5} \mathrm{~A}$ better understanding of patients' preferences for treatment and involving patients in clinical decision making could also help to optimize disease management and medication adherence.

Several methods have been developed and used to assess preferences in health care, ranging from simple rating scale exercises to more complex forms of preference valuation techniques such as conjoint analysis. Conjoint analysis is a decomposition method that derive the implicit values for factors (or attributes and levels) from some overall score for a profile consisting of two or more factors. ${ }^{6,7}$ In particular, the use of traditional discretechoice experiments (DCEs), has drastically increased in recent years. ${ }^{2,8}$ Although DCEs may take many forms, this study refers solely to the 'traditional' DCE in which a respondent typically chooses one alternative among a number of competing multi-profile options described by varied factors. Another type of conjoint analysis, namely best-worst scaling (BWS), is becoming increasingly popular in healthcare. ${ }^{9}$ BWS devised by Finn and Louviere, ${ }^{10}$ first applied in health care by Szeinbach et al. in which it was named a maximum difference conjoint analysis ${ }^{11}$ and formally introduced to health care research by McIntosh and Louviere, ${ }^{12}$ differs from traditional DCEs, mainly because it elicits additional information on the least preferred option. ${ }^{13}$ BWS consists of choice tasks, with a minimum of 3 items/options, in which a person is asked to indicate the best and the worst item/option, with the overall aim to obtain a full ranking of items in a manner that is easy for respondents and can then be analysed in various ways.

As health care applications of BWS emerged only recently, it is important to reflect on and assess current practice. Several reviews of DCEs have been conducted ${ }^{14,15}$ and revealed trends toward the conduct and the use of DCEs. For BWS studies, although not systematically reported, scholars noted its increasing popularity in health care. ${ }^{13,16}$ Yet, little is known about current practice and trends in the use of BWS in health care, except from two recent overview articles of BWS studies of Mühlbacher et al. ${ }^{17,18}$ These articles provide insights into the possibilities of application, the theoretical concepts underlying the BWS method, analytic methods, strengths and weakness of BWS cases and the list of studies that applied the BWS method. However, these studies do not report on the current 
practice per BWS cases, on the quality appraisal of BWS studies, and on trends of BWS studies in health care. Reviewing the use of BWS and reflecting on current practice could help in improving best practices of BWS and potentially lead to an increased interest of policy makers or physicians to the wide usability and value of BWS. Hence, we conducted a systematic review to identify and analyse all BWS conducted in health care, and to identify if key aspects of BWS have changed over time.

\section{Best worst scaling}

BWS can be used to determine preferences for a wide range of health care questions, by asking the respondent to indicate the best and the worst in a set of available items or options. There are three types of BWS that differ in the complexity of the items or options under considerations: the BWS object case (or case 1) the BWS profile case (or case 2), and the BWS multi-profile case (or case 3). ${ }^{16}$

In the BWS object case, the researcher is interested in the relative values associated with each of a list of objects. Figure 5.1 a illustrates the case of three relevant attributes. Once the researcher has chosen the list of objects, the researcher presents choice sets of these to respondents to obtain best and worst data. Here the objects are not decomposed into factors. BWS object case can be used, for instance, to examine features of a new medicine. Hence, one needs to develop a list of mutually exclusive objects. ${ }^{19}$ The object case BWS can be very attractive in health care because health care goods/services can be complicated and even pairs of specifications (e.g. a simple DCE) may lead to unacceptable cognitive burden, particularly among vulnerable patient groups. ${ }^{16}$

In the BWS profile case, in contrast with BWS object case, the level of each attribute is shown and the choice set has the structure of a single profile. In Figure $5.1 \mathrm{~b}$ a possible treatment is characterised by five attributes, providing its levels in each choice-set. However, the respondent is not required to consider the value of the profile as a whole, but has to consider the factor levels that describe it, and choose the one that is best (most attractive) and the one that is worst (least attractive). Hence, profiles must describe particular configurations or specifications of a good or service, in which each profile represents a combination of factors and associated levels. ${ }^{19}$ The factors are fixed across all profiles but the combinations of factor levels differ by profile.

In contrast to BWS profile case, the BWS multi-profile case provides multiple profiles and the respondent is asked to choose the best (most attractive) profile as well as the worst (least attractive) one in the current choice set. Figure 5.1c illustrates a choice set, using again a possible treatment as an example. Respondents base their choice on the whole scenario instead of single factor levels as in BWS profile case The multiple profiles include all the factors and one level per factor and the respondent is assumed to make deliberate choices based on presented levels for each factor. The BWS multi-profile case is most similar to a traditional DCE but extends the general DCE design to allow for best and worst choices. $^{19}$ 


\begin{tabular}{|c|c|c|}
\hline Least important & Factors of medicine & Most important \\
\hline $\mathrm{X}$ & Side-effects & $\mathrm{X}$ \\
\hline & Frequency of intake & \\
\hline & Mode of administration & \\
\hline
\end{tabular}

Figure 5.1 a Example of a BWS object case

\begin{tabular}{|c|c|c|}
\hline Least important & Factors of medicine & Most important \\
\hline & Side effects: moderate & \\
\hline & Frequency of intake: daily & $X$ \\
\hline$X$ & Mode of administration: oral supplement & \\
\hline & Out-of-pocket expenses: $€ 10$ per week & \\
\hline & Duration of treatment: eight weeks & \\
\hline
\end{tabular}

Figure 5.1b Example of a BWS profile case

\begin{tabular}{|l|l|l|l|}
\hline Factors & Drug A & Drug B & Drug C \\
\hline Side effects: & Moderate & Severe & Severe \\
\hline Frequency of intake: & Daily & Monthly & Monthly \\
\hline Mode of administration: & Oral supplement & Injection & Injection \\
\hline Out-of-pocket expenses: & $€ 10$ per week & $€ 15$ per week & $€ 5$ per week \\
\hline Duration of treatment: & Eight weeks & Twelve weeks & Four weeks \\
\hline $\begin{array}{l}\text { Which drug would be the } \\
\text { best treatment for you? }\end{array}$ & & & \\
\hline $\begin{array}{l}\text { Which drug would be the } \\
\text { worst treatment for you? }\end{array}$ & $\square$ & & \\
\hline
\end{tabular}

Figure 5.1c Example of a BWS multi-profile case

\section{Methods}

\subsection{Literature search}

A systematic review was conducted, using MEDLINE (via PubMed) and EMBASE to identify all English language BWS studies available in print or online between January 1990 and April 2016. In addition, bibliographies of BWS applications identified in the literature search were manually searched (forward and backward) for additional articles. Following examples of DCE reviews from Ryan et al. ${ }^{2}$ and Clark et al., ${ }^{14}$ the search strategy was formulated according to free text terms. The following free text terms focusing on BWS studies were used individually in each database: 'object scaling' OR 'BWS' OR 'best worst scaling' OR 'best worst' OR 'max diff' OR 'maxdiff' OR 'max diff scaling', 'maxdiff scaling' OR 'maximum difference scaling' (see Electronic Supplementary Material S5.1). The research team discussed and approved the list of free text terms. In addition, we have cross-checked the identified articles with the articles identified by a recent review of Mühlbacher et al. ${ }^{17}$ 


\subsection{Selection of studies}

Titles and abstracts were screened independently by two researchers (KLC \& BW). Studies were included if they were original BWS studies, published as a full text article, and applied to health care. The definition of Louviere et al. ${ }^{19}$ was used for inclusion of BWS studies, meaning only BWS studies were included in which respondents needed to indicate both a best and worst option per choice-task. Hence, studies looking at best or worst alternatives only as well as studies using only best data to estimate models were excluded. Furthermore, studies based on simulated data were excluded. Disagreements about inclusion or exclusion based on abstract review were determined by consensus. At least two independent reviewers (KLC, BW, ILH, EJ) then reviewed the full text of all remaining articles. Consensus on study inclusion was reached by at least two reviewers.

\subsection{Data extraction}

For each article that met our inclusion criteria, two independent reviewers (KLC \& EJ, and BW \& ILH) systematically extracted data using a predefined extraction form that included a variety of data elements relevant to the conduct of BWS experiments. Any disagreements were resolved by consensus. This data extraction form was based on a review by $\mathrm{De}$ Bekker et al. ${ }^{20}$ on DCE and was further developed through discussion within the research team. A pilot study was performed by four reviewers for four studies which led to some adjustments to the data extraction form. The extracted data included three categories: general study information, design and choice sets and estimation procedure and validity. General study information included: authors, year of publication, study name, study type, study setting, target population, type of case, and area of application. Design and choice sets included: sample size, methods for factor selection, type of BWS, design of BWS, domains of BWS factors, number of best-worst options per choice set, number of included factors, maximum number of choice tasks, number of factors per choice task, BWS survey administration, and BWS survey method. Estimation procedure and validity included: analytical method, use of software packages to analyse BWS experiments, preference heterogeneity taken into account (by means of latent class or random parameters analyses), subgroup analysis, use of pilot validity checks (i.e. test-retest, face validity of a dominant choice or subgroup analysis).

\subsection{Quality assessment}

In order to assess the study quality, the Purpose, Respondents, Explanation, Findings, Significance (PREFS) checklist $^{21}$ was used. The PREFS was specifically developed based on guidelines for conjoint analyses, patient reported outcomes and randomized trials to assess studies reporting on factors that could influence the validity of findings. To the best of our knowledge, this is the only checklist available for estimating the quality of preference studies. ${ }^{21}$ PREFS assesses five criteria as acceptable (1) or unacceptable (0): purpose of the study (is the purpose of the study in relation to preferences clearly stated?); respondent 
sampling (are the responders similar to the non-responders?); explanation of preference assessment methods (are methods of assessing preferences clearly explained?); findings reported for total sample (were all respondents included in the reported findings and analysis of preference results?); and significance testing (were significance tests used to assess the preference results?). The five criteria are summed to indicate an overall quality score ranging from 0 to 5 . Studies were independently scored by two reviewers and disagreements were resolved by consensus in pairs of two researchers (KLC \& EJ, and BW \& $\mid \mathrm{H})$.

\subsection{Data analysis}

Extracted data were grouped by BWS cases. Additionally, for several items (sample size, number of included factors, maximum number of choice tasks, number of factors per choice task and analytical method), we reported medians and ranges for different time periods (i.e. $\leq 2010,2011,2012,20132014$ and 2015) to investigate potential trends. Extracted data are presented in tables, separately for the three different BWS cases.

\section{Results}

\subsection{Article inclusion}

Initial database searches resulted in 632 articles $(538$ from Pubmed and 94 from EMBASE). This corresponded to 588 unique studies of which 69 were eligible for full text review. Full text review resulted in the exclusion of 11 studies as six studies did not perform a BWS experiment, one study had no application in health care, two studies were not original research, one study included thinking aloud (qualitative) results only, and another study did not report on the results. Figure 5.2 shows the flow chart of the article identification process.

\subsection{General study information}

A total of 62 BWS studies were identified (58 articles) of which 26 were BWS object case studies, 29 were BWS profile case studies, and seven were BWS multi-profile case studies. Results indicated an increase in the number of BWS object case and BWS profile case studies applied in health care over time (see Figure 5.3). The median number of participants was 175 for BWS object case, 162 for BWS profile case, and 326 for BWS multi-profile case. Most studies were conducted in the United States (67\%) for BWS object case, and in Australia (36\%) and United Kingdom (32\%) for BWS profile case (see Table 5.1). 


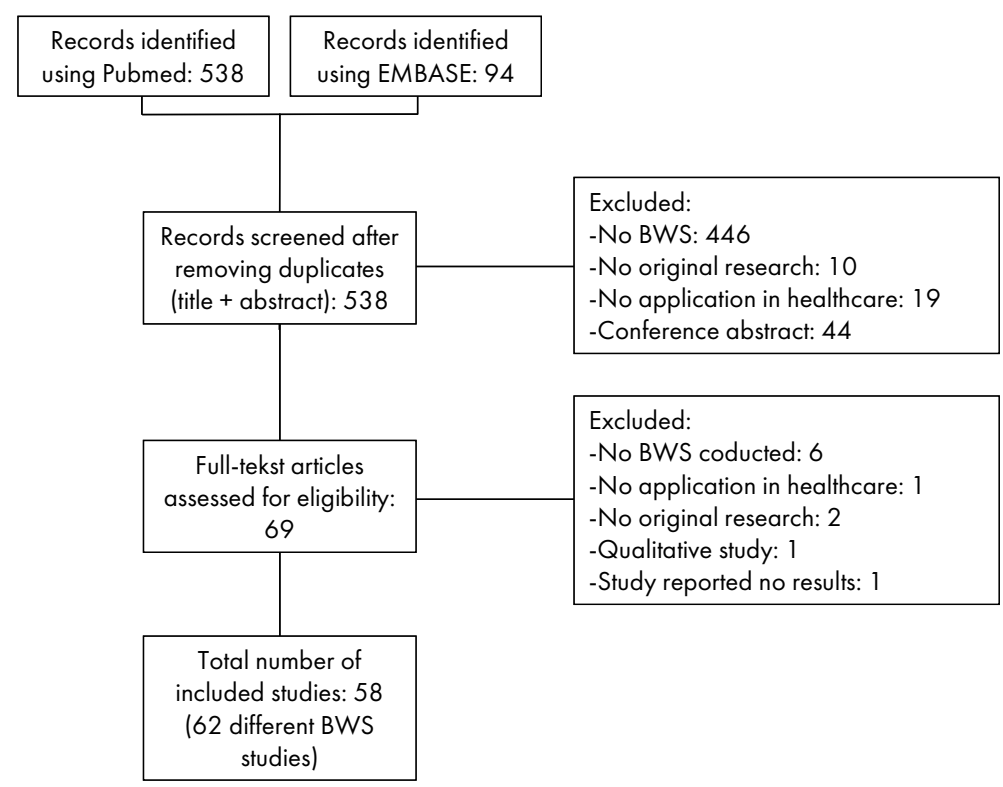

Figure 5.2 Flow chart studies identification

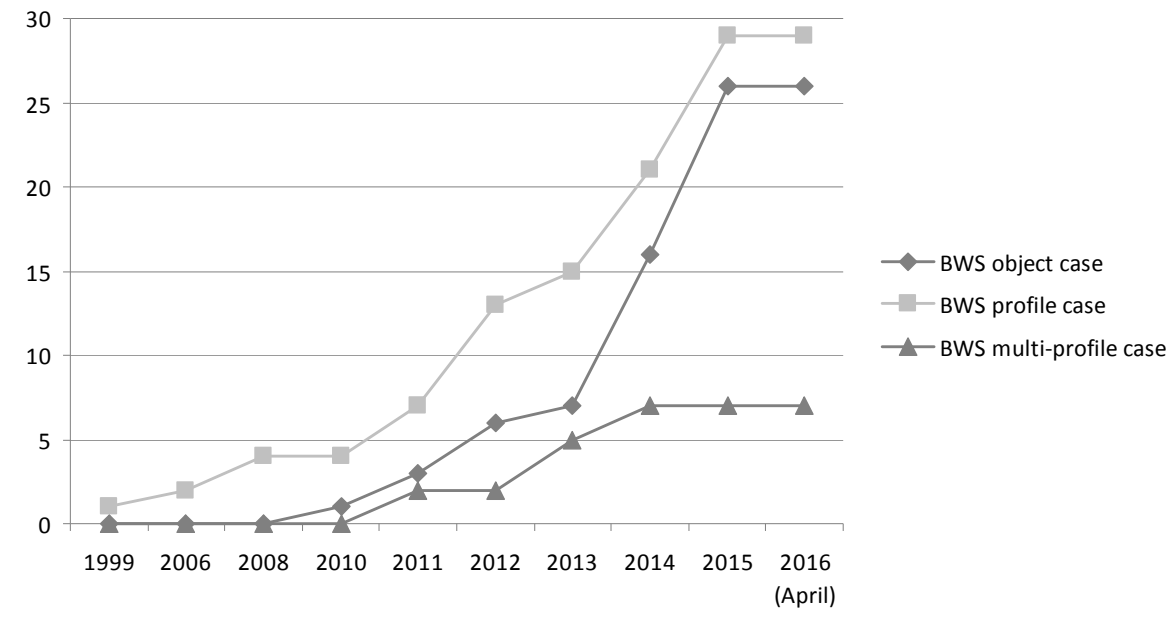

Figure 5.3 Cummulative number of BWS studies by year and per BWS case 


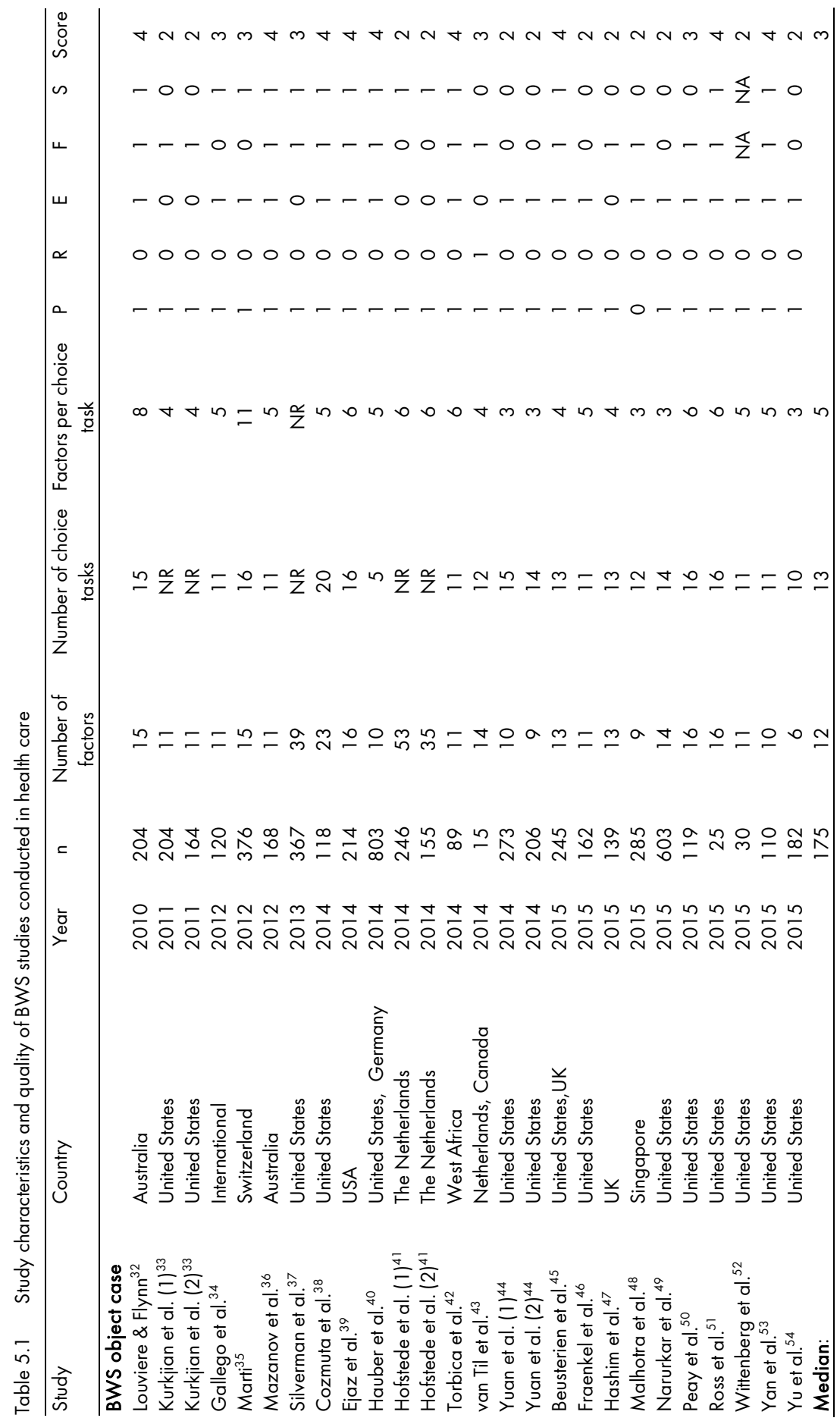




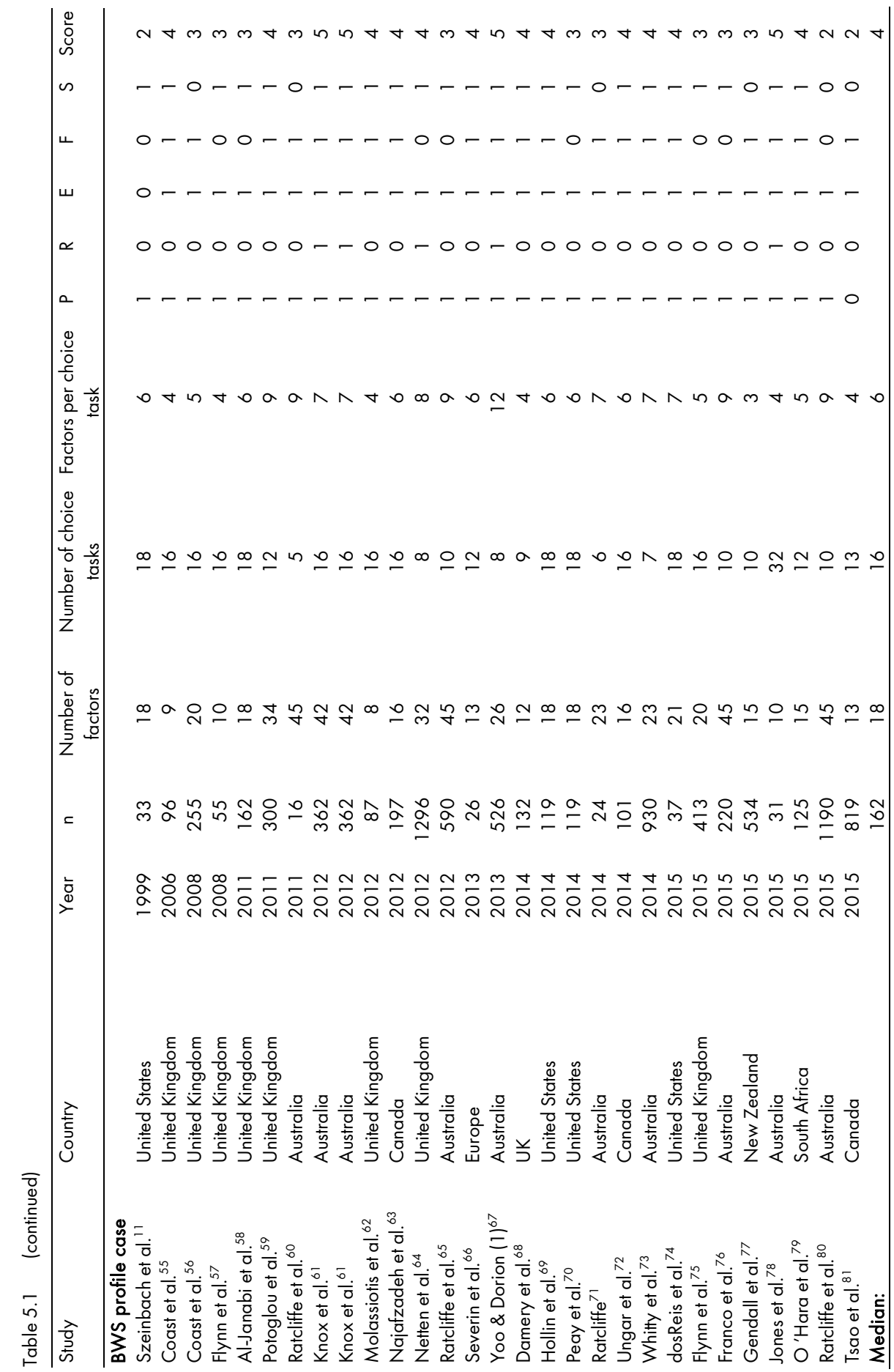




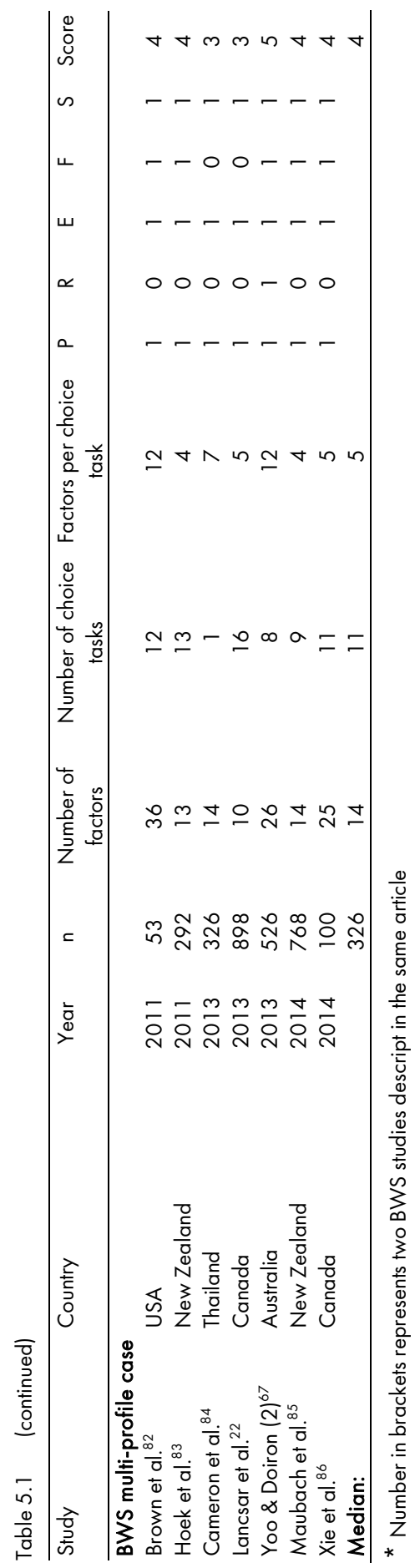


Most studies had health care professionals (BWS object case 27\%; BWS profile case $16 \%$; BWS multi-profile case 13\%), patients (BWS object case $27 \%$; BWS profile case 29\%; BWS multi-profile case 13\%), and the general population (BWS object case $27 \%$; BWS profile case $39 \%$; BWS multi-profile case $38 \%$ ) as the target population. The main focus of all cases was to explore patient and consumer experience factors (BWS object case $32 \%$; BWS profile case $45 \%$; BWS multi-profile case $40 \%$, see Table 5.2 ). A table including a detailed outline of study characteristics can be found in the Electronic Supplementary Material S5.2.

Table 5.2 Characteristics of BWS studies conducted in health care

\begin{tabular}{|c|c|c|c|c|c|c|c|}
\hline \multirow[t]{2}{*}{ Item } & \multirow[t]{2}{*}{ Category } & \multicolumn{2}{|c|}{$\begin{array}{l}\text { BWS object } \\
\text { case }\end{array}$} & \multicolumn{2}{|c|}{ BWS profile case } & \multicolumn{2}{|c|}{$\begin{array}{l}\text { BWS multi-profile } \\
\text { case }\end{array}$} \\
\hline & & $\mathrm{n}$ & Percentage & $\mathrm{n}$ & Percentage & $n$ & Percentage \\
\hline \multirow{6}{*}{$\begin{array}{l}\text { Target } \\
\text { population* }\end{array}$} & Health care professionals & 7 & $27 \%$ & 5 & $16 \%$ & 1 & $13 \%$ \\
\hline & Patients & 7 & $27 \%$ & 9 & $29 \%$ & 1 & $13 \%$ \\
\hline & General population & 7 & $27 \%$ & 12 & $39 \%$ & 3 & $38 \%$ \\
\hline & (Informal) Caregivers & 3 & $12 \%$ & 4 & $13 \%$ & 1 & $13 \%$ \\
\hline & Policy makers & 1 & $4 \%$ & 0 & $0 \%$ & 0 & $0 \%$ \\
\hline & Other stakeholders & 1 & $4 \%$ & 1 & $3 \%$ & 2 & $25 \%$ \\
\hline \multirow{7}{*}{$\begin{array}{l}\text { Area of } \\
\text { application* }\end{array}$} & Valuing health outcomes & 10 & $32 \%$ & 13 & $45 \%$ & 4 & $40 \%$ \\
\hline & $\begin{array}{l}\text { Investigating trade-offs between } \\
\text { health outcomes and patient or } \\
\text { consumer experience factors }\end{array}$ & 8 & $26 \%$ & 4 & $14 \%$ & 0 & $0 \%$ \\
\hline & $\begin{array}{l}\text { Estimating utility weights within the } \\
\text { quality-adjusted life-year } \\
\text { framework }\end{array}$ & 2 & $6 \%$ & 1 & $3 \%$ & 4 & $40 \%$ \\
\hline & Job choices & 0 & $0 \%$ & 3 & $10 \%$ & 1 & $10 \%$ \\
\hline & $\begin{array}{l}\text { Developing priority setting } \\
\text { mechanism }\end{array}$ & 0 & $0 \%$ & 1 & $3 \%$ & 1 & $10 \%$ \\
\hline & $\begin{array}{l}\text { Health professionals preferences } \\
\text { for treatment and screening options }\end{array}$ & 4 & $13 \%$ & 4 & $14 \%$ & 0 & $0 \%$ \\
\hline & Other & 6 & $19 \%$ & 1 & $3 \%$ & 0 & $0 \%$ \\
\hline \multirow[t]{7}{*}{ Design BWS } & Orthogonal main effects design & 3 & $12 \%$ & 19 & $66 \%$ & 3 & $43 \%$ \\
\hline & $\begin{array}{l}\text { Latin square (balanced order and } \\
\text { pairing) design }\end{array}$ & 2 & $8 \%$ & 0 & $0 \%$ & 0 & $0 \%$ \\
\hline & Balanced incomplete block design & 14 & $54 \%$ & 2 & $7 \%$ & 1 & $14 \%$ \\
\hline & Full factorial & 2 & $8 \%$ & 2 & $7 \%$ & 0 & $0 \%$ \\
\hline & Bayesian efficient design & 1 & $4 \%$ & 2 & $7 \%$ & 1 & $14 \%$ \\
\hline & Fractional other & 0 & $0 \%$ & 2 & $7 \%$ & 1 & $14 \%$ \\
\hline & NR & 4 & $15 \%$ & 2 & $7 \%$ & 1 & $14 \%$ \\
\hline \multirow{6}{*}{$\begin{array}{l}\text { Domains of BWS } \\
\text { factors* }\end{array}$} & Money & 8 & $12 \%$ & 9 & $13 \%$ & 4 & $27 \%$ \\
\hline & Time & 6 & $9 \%$ & 7 & $10 \%$ & 1 & $7 \%$ \\
\hline & Risk & 7 & $11 \%$ & 6 & $8 \%$ & 2 & $13 \%$ \\
\hline & Health care & 16 & $24 \%$ & 17 & $24 \%$ & 3 & $20 \%$ \\
\hline & Health status & 15 & $23 \%$ & 16 & $22 \%$ & 2 & $13 \%$ \\
\hline & Other & 14 & $21 \%$ & 17 & $24 \%$ & 3 & $20 \%$ \\
\hline
\end{tabular}


Table 5.2 (continued)

\begin{tabular}{|c|c|c|c|c|c|c|c|}
\hline \multirow[t]{2}{*}{ Item } & \multirow[t]{2}{*}{ Category } & \multicolumn{2}{|c|}{$\begin{array}{l}\text { BWS object } \\
\text { case }\end{array}$} & \multicolumn{2}{|c|}{ BWS profile case } & \multicolumn{2}{|c|}{$\begin{array}{l}\text { BWS multi-profile } \\
\text { case }\end{array}$} \\
\hline & & $\mathrm{n}$ & Percentage & $\mathrm{n}$ & Percentage & $\mathrm{n}$ & Percentage \\
\hline \multirow{10}{*}{$\begin{array}{l}\text { Analytical } \\
\text { method* }^{*}\end{array}$} & Hierarchical Bayes & 7 & $21 \%$ & 1 & $3 \%$ & 0 & $0 \%$ \\
\hline & $\begin{array}{l}\text { Simple summary statistics (Best- } \\
\text { minus worst summary statistics) }\end{array}$ & 11 & $32 \%$ & 8 & $24 \%$ & 0 & $0 \%$ \\
\hline & MNL model & 4 & $12 \%$ & 16 & $47 \%$ & 5 & $63 \%$ \\
\hline & Weighted least squares & 2 & $6 \%$ & 5 & $15 \%$ & 1 & $13 \%$ \\
\hline & Latent class analysis & 3 & $9 \%$ & 1 & $3 \%$ & 0 & $0 \%$ \\
\hline & Max diff scaling & 2 & $6 \%$ & 0 & $0 \%$ & 2 & $25 \%$ \\
\hline & Ordered Logit & 3 & $9 \%$ & 0 & $0 \%$ & 0 & $0 \%$ \\
\hline & Random parameter logit model & 0 & $0 \%$ & 1 & $3 \%$ & 0 & $0 \%$ \\
\hline & $\begin{array}{l}\text { Qualitative; thinking aloud } \\
\text { procedure }\end{array}$ & 2 & $6 \%$ & 0 & $0 \%$ & 0 & $0 \%$ \\
\hline & NR & 7 & $21 \%$ & 1 & $3 \%$ & 0 & $0 \%$ \\
\hline \multirow[t]{4}{*}{ Heterogeneity } & No heterogeneity & 17 & $68 \%$ & 19 & $66 \%$ & 4 & $57 \%$ \\
\hline & Latent class & 2 & $8 \%$ & 6 & $21 \%$ & 0 & $0 \%$ \\
\hline & Random parameter & 4 & $16 \%$ & 4 & $14 \%$ & 3 & $43 \%$ \\
\hline & NR & 2 & $8 \%$ & 0 & $0 \%$ & 0 & $0 \%$ \\
\hline \multirow{7}{*}{$\begin{array}{l}\text { Software for } \\
\text { analyses of BWS* }\end{array}$} & Sawtooth software & 7 & $27 \%$ & 2 & $7 \%$ & 0 & $0 \%$ \\
\hline & SAS & 2 & $8 \%$ & 3 & $10 \%$ & 2 & $29 \%$ \\
\hline & Stata & 2 & $8 \%$ & 9 & $31 \%$ & 1 & $14 \%$ \\
\hline & Nlogit & 0 & $0 \%$ & 3 & $10 \%$ & 0 & $0 \%$ \\
\hline & SPSS & 2 & $8 \%$ & 0 & $0 \%$ & 0 & $0 \%$ \\
\hline & Latent Gold Choice & 0 & $0 \%$ & 2 & $7 \%$ & 0 & $0 \%$ \\
\hline & $\mathrm{NR} / \mathrm{NA}$ & 13 & $50 \%$ & 10 & $34 \%$ & 4 & $57 \%$ \\
\hline
\end{tabular}

* more than one category per BWS study possible. MNL: Multinomial logistic regression. Max Diff: Maximum difference scaling. NR: Not reported. NA: Not applicable

\subsection{Design and choice sets}

Looking at current practice regarding designs and choice sets used in BWS studies, most BWS studies selected factors (attributes and levels) using literature reviews (BWS object case $33 \%$; BWS profile case 35\%; and BWS multi-profile case 50\%) and/or qualitative methods such as interviews and focus groups (BWS object case $55 \%$; BWS profile case $59 \%$; and BWS multi-profile case $25 \%$ ). For BWS object case, the median number of factors was 12 while the average number of factors in BWS profile case (defined as the number of levels included in the experiment) was 18. For the BWS multi-profile case the median number of factors was 14. The median number of choice sets for BWS object case was 13, for BWS profile case 16, and BWS multi-profile case 11. Almost all studies only asked one best and one worst option. Only one study (BWS multi-profile case) asked respondents to identify two best and two worst choices for each choice set. ${ }^{22}$

In most studies, the survey was administrated in a self-completion format (BWS object case 74\%; BWS profile case 72\%; and BWS multi-profile case $71 \%$ ) and many studies used an online platform (BWS object case $48 \%$; BWS profile case $41 \%$; and BWS multi- 
profile case $29 \%$ ). The most used design by far is the balanced incomplete block design for BWS object case (54\%), and the orthogonal main effects design for BWS profile case $(66 \%)$ and BWS multi-profile case (43\%). However, some studies did not report on their design (BWS object case 15\%; BWS profile case 7\%; and BWS multi-profile case $14 \%$ ).

\subsection{Estimation procedure and validity}

As shown in Table 5.2, BWS object case studies most often employed Hierarchical Bayes $(21 \%)$ and simple summary statistics (32\%). BWS profile case and BWS multi-profile case studies used the multinomial logistic (MNL) model (in $47 \%$ and $63 \%$ of the studies respectively). Several studies did not report on the software used for analyses (BWS object case $50 \%$; for BWS profile case $34 \%$; and BWS multi-profile case $57 \%$ ). Of those that reported the software used for analysis, Sawtooth software was most frequently used for BWS object case $(27 \%)$, while STATA software was most frequently used in the analyses of BWS profile case (31\%) and SAS was most frequently reported in the BWS multi-profile case studies (29\%). Most studies did not take into account heterogeneity (BWS object case $68 \%$; BWS profile case $66 \%$; and BWS multi-profile case $57 \%$ ). To validate the factors, a pilot study was used for 15 BWS object case studies (58\%), 16 BWS profile case studies (55\%), and 3 BWS multi-profile case studies (43\%). In addition, a large majority of BWS studies conducted no validity checks (BWS object case $81 \%$; BWS profile case $76 \%$; and BWS multi-profile case $71 \%$ ).

\subsection{Trend analysis}

Table 5.3 depicts the trend analysis, which indicated a decreasing median sample size since 2013 for BWS object case, from $n=367$ in 2013 to $n=151$ in articles published in 2015. For BWS profile case and BWS multi-profile case, the trends are not so clear which is also depicted in the large range around the median. Moreover, there appears to be a decrease in the median number of factors included in BWS object case studies since 2013. For the BWS profile case a similar trend was found since 2011. For BWS multi-profile case, the trend is not so clear. For BWS profile case, a decrease in the median number of factors per choice task was found since 2013. No trends can be found regarding the median number of choice tasks and the median number of factors per choice task in BWS studies. However, looking at the maximum values of the number of choice tasks for BWS profile there seems to be a slight increase from 2010 onwards. Trends of the analytical methods are depicted in Figure 5.4a, 5.4b, and 5.4c. There is a steep increase in the use of best-minus worst counting and Hierarchical Bayes for BWS object case studies (see Figure 5.4a). For the BWS profile case, a steep increase was observed in the use of MNL models (see Figure 5.4b). Regarding BWS multi-profile case studies, trends were not clear (see Figure 5.4c). 
Table 5.3 Trend analysis

\begin{tabular}{|c|c|c|c|c|c|c|c|c|c|c|c|c|}
\hline & \multicolumn{4}{|c|}{ BWS object case } & \multicolumn{4}{|c|}{ BWS profile case } & \multicolumn{4}{|c|}{ BWS multi-profile case } \\
\hline & Mdn & Min & Max & $\mathrm{N}$ & Mdn & Min & Max & $\mathrm{N}$ & Mdn & Min & Max & $\mathrm{N}$ \\
\hline \multicolumn{13}{|c|}{ Number of included factors } \\
\hline$\leq 2010$ & 15 & 15 & 15 & 1 & 14 & 9 & 20 & 4 & 0 & 0 & 0 & 0 \\
\hline 2011 & 11 & 11 & 11 & 2 & 34 & 18 & 45 & 3 & 24.5 & 13 & 36 & 2 \\
\hline 2012 & 11 & 11 & 15 & 3 & 37 & 8 & 45 & 6 & 0 & 0 & 0 & 0 \\
\hline 2013 & 39 & 39 & 39 & 1 & 19.5 & 13 & 26 & 2 & 14 & 10 & 26 & 3 \\
\hline 2014 & 14 & 9 & 53 & 9 & 18 & 12 & 23 & 6 & 19.5 & 14 & 25 & 2 \\
\hline 2015 & 12 & 6 & 16 & 10 & 17.5 & 10 & 45 & 8 & 0 & 0 & 0 & 0 \\
\hline \multicolumn{13}{|c|}{ Number of factors per choice task } \\
\hline$\leq 2010$ & 8 & 8 & 8 & 1 & 4.5 & 4 & 6 & 4 & 0 & 0 & 0 & 0 \\
\hline 2011 & 4 & 4 & 4 & 2 & 9 & 6 & 9 & 3 & 8 & 4 & 12 & 2 \\
\hline 2012 & 5 & 5 & 11 & 3 & 7 & 4 & 9 & 6 & 0 & 0 & 0 & 0 \\
\hline 2013 & NR & NR & NR & 1 & 9 & 6 & 12 & 2 & 7 & 5 & 12 & 3 \\
\hline 2014 & 5 & 3 & 6 & 9 & 6 & 4 & 7 & 6 & 4.5 & 4 & 5 & 2 \\
\hline 2015 & 4.5 & 3 & 6 & 10 & 5 & 3 & 9 & 8 & 0 & 0 & 0 & 0 \\
\hline \multicolumn{13}{|c|}{ Number of choice tasks } \\
\hline$\leq 2010$ & 15 & 15 & 15 & 1 & 16 & 16 & 18 & 4 & 0 & 0 & 0 & 0 \\
\hline 2011 & NR & NR & NR & 2 & 12 & 5 & 18 & 3 & 12.5 & 12 & 13 & 2 \\
\hline 2012 & 11 & 11 & 16 & 3 & 16 & 8 & 16 & 6 & 0 & 0 & 0 & 0 \\
\hline 2013 & NR & NR & NR & 1 & 10 & 8 & 12 & 2 & 8 & 1 & 16 & 3 \\
\hline 2014 & 14 & 5 & 20 & 9 & 12.5 & 6 & 18 & 6 & 10 & 9 & 11 & 2 \\
\hline 2015 & 12.5 & 10 & 16 & 10 & 12.5 & 10 & 32 & 8 & 0 & 0 & 0 & 0 \\
\hline \multicolumn{13}{|c|}{ Sample size } \\
\hline$\leq 2010$ & 204 & 204 & 204 & 1 & 75.5 & 33 & 255 & 4 & 0 & 0 & 0 & 0 \\
\hline 2011 & 184 & 164 & 204 & 2 & 162 & 16 & 300 & 3 & 172.5 & 53 & 292 & 2 \\
\hline 2012 & 168 & 120 & 376 & 3 & 362 & 87 & 1296 & 6 & 0 & 0 & 0 & 0 \\
\hline 2013 & 367 & 367 & 367 & 1 & 276 & 26 & 526 & 2 & 526 & 326 & 898 & 3 \\
\hline 2014 & 206 & 15 & 803 & 9 & 119 & 24 & 930 & 6 & 434 & 100 & 768 & 2 \\
\hline 2015 & 150.5 & 25 & 603 & 10 & 316.5 & 31 & 1190 & 8 & 0 & 0 & 0 & 0 \\
\hline
\end{tabular}

Mdn: Median. Min: Minimum. Max: Maximum

\subsection{Quality of BWS studies}

Results of the PREFS checklist to assess the quality of current BWS studies are included in Table 5.1. We found that $98 \%$ of the BWS studies had a preference-related purpose clearly stated. However, most studies did not report whether the responders were similar to non-responders, with 56 studies $(91 \%)$ categorised as unacceptable regarding this criterion. We found that 54 studies (86\%) clearly explained methods of assessing preferences. Most studies included all respondents in the analyses of preference results and reported findings (65\%). Furthermore, 44 studies (71\%) used significance tests to assess the preference results. Regarding the total PREFS score, half of the studies scored a 4 or higher $(56.4 \%)$, of which only 5 studies achieved a score of 5 . 
a

BWS object case

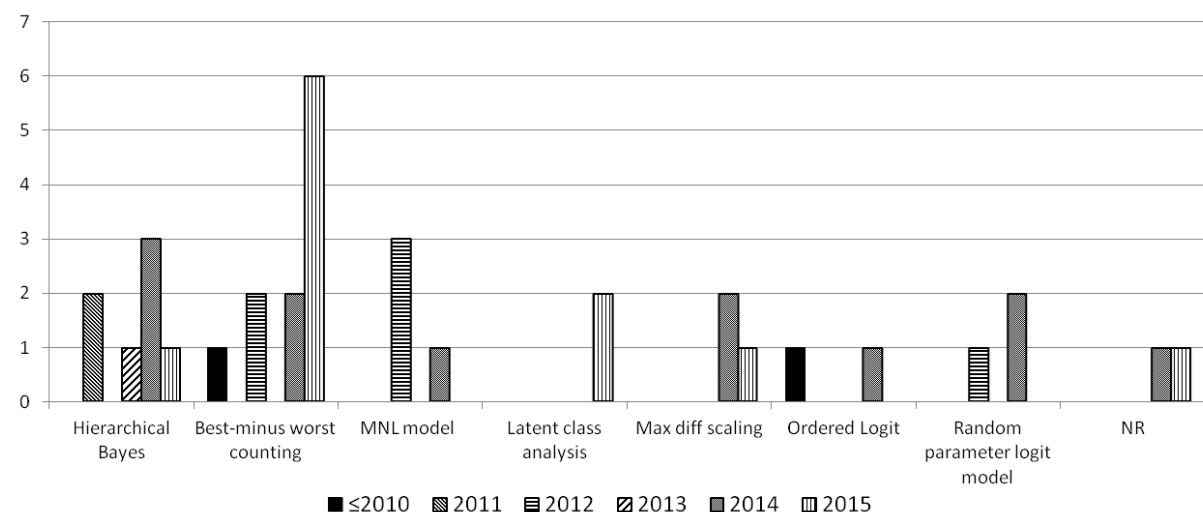

b

BWS profile case

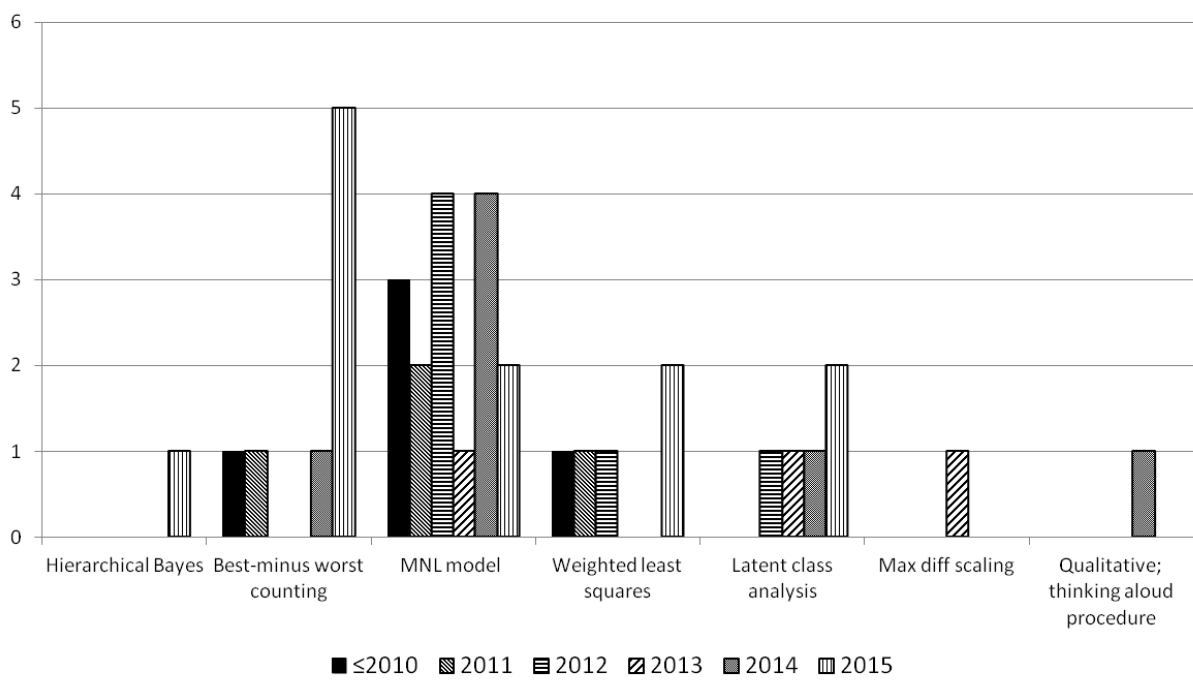


C

\section{BWS multi-profile case}

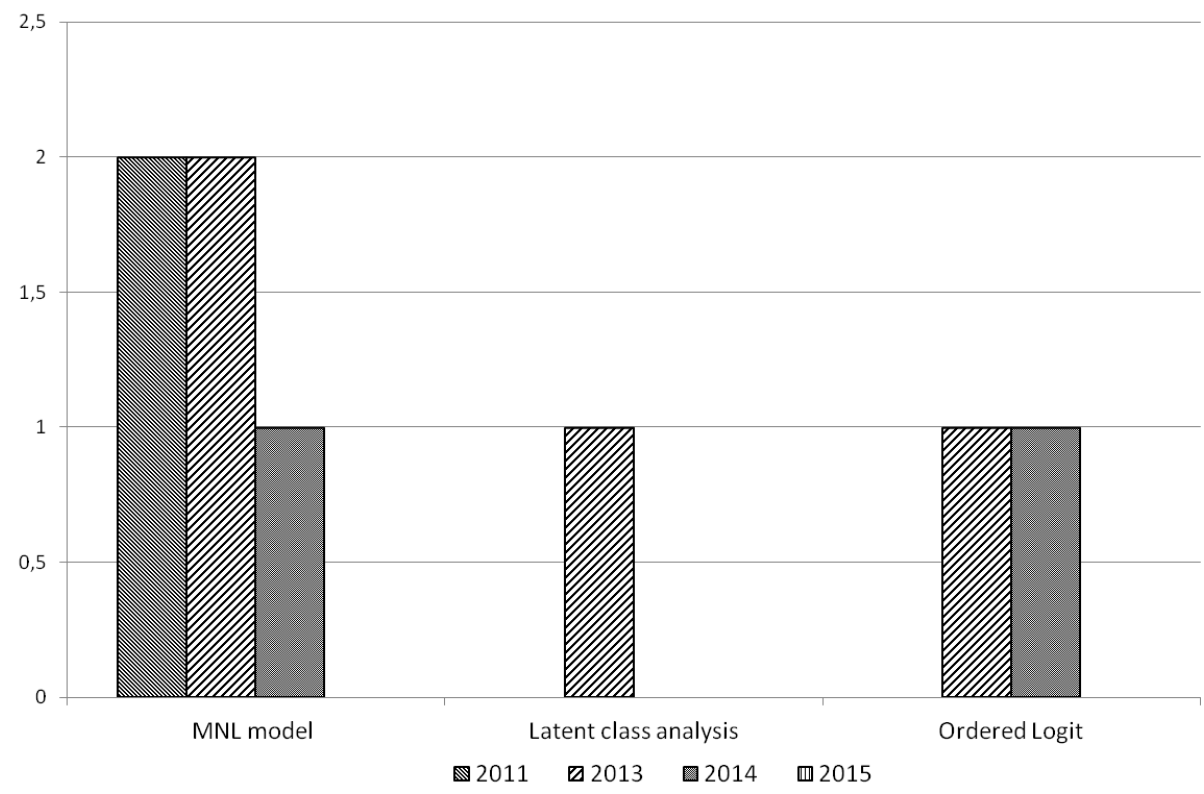

Figure 5.4 a. Analytical method used per year: BWS object case: MNL: Multinomial logistic regression; Max Diff: Maximum difference scaling; NR: Not reported; b. Analytical method used per year: BWS profile case; MNL: Multinomial logistic regression; Max Diff: Maximum difference scaling; NR: Not reported; c. Analytical method used per year: BWS multi-profile case. MNL: Multinomial logistic regression; Max Diff: Maximum difference scaling; NR: Not reported

\section{Discussion}

This study reviewed current practice in the use and conduct of BWS within health care. The use of BWS, and especially BWS object case and BWS profile case, has drastically increased in health care, with a steep increase in the last two years on a wide range of applications (e.g. valuing health outcomes and investigating preferences between health outcomes and patient or consumer experience factors) and populations (e.g. health care professionals, patients, general population, and caregivers). This increase of published BWS studies may indicate an increasing awareness of advantages of the BWS method to elicit preferences in health care. It is apparent that BWS, especially the object case, is capable of incorporating a large set of items or factors to elicit preferences compared to DCE. In DCE, the average number of included factors was estimated to be 4 to $6,{ }^{14}$ compared to an average of $17(M d n=12)$ factors for BWS object cases. BWS object case can be especially useful if one is interested in a list of items that are difficult to reduce to a number acceptable for other conjoint analyses such as a DCE. Additionally, compared to 
the DCE, BWS (especially BWS object cases and BWS profile cases) may be less cognitively burdensome, which can be valuable in health care settings where preferences are often elicited from vulnerable patient groups. ${ }^{9}$ For example, it has been stated that best-worst tasks take advantage of a person's propensity to identify and respond more consistently to extreme options and BWS tasks seem easier to understand for people. ${ }^{9,23}$ Compared to results from a DCE, BWS may give different findings especially due to choosing a "worst" may be a different psychological process. The literature has shown mixed results for whether similar patterns arise between the two methods and further comparison would be needed. ${ }^{23-25}$ Hence, one should be careful when directly comparing the results of DCEs and BWS. Despite the observed increase in the use of BWS within healthcare, the number of published BWS studies still lags behind the number of DCEs. Clark et al. ${ }^{14}$ identified 179 DCEs published between 1990 and 2012, which is more than three time higher than the 58 articles identified in this paper until April 2016.

Compared to BWS object case and BWS profile case, a notable difference in the use of BWS multi-profile case within health care was observed. From this review, it is apparent that there is a limited application of BWS multi-profile case, which may have several explanations. One explanation is that researchers have difficulty differentiating BWS multiprofile case from a DCE, or fail to see added value in using a BWS multi-profile case over a DCE. There are only minor differences between both methods, which may explain that the BWS multi-profile case is often referred to as a BWS DCE. ${ }^{26,27}$ Although the BWS multi-profile case has been stated to offer distinct advantages, such as to provide richer information than methods based on 'pick one' approach ${ }^{13,27}$ (e.g. resulting in a smaller sample size needed), the need to elicit the best and worst alternative has the limitation of comparing a minimum of three profiles per choice task.

In our review, several trends about changes in key aspects of BWS have been identified. First, regarding the sample size of BWS object case, we observed smaller sample sizes since 2013. Literature provides no guidance to the minimal sample size for a BWS application. Future research should focus on developing sample size calculations based on the desired statistical power, similar to DCEs. ${ }^{28}$ Second, the median number of included factors substantially decreases in BWS object case and BWS profile case since respectively 2013 and 2011 . For DCEs, literature indicates no clear trends regarding the number of factors used. ${ }^{14}$ Yet, this study revealed a trend of a decreasing number of factors included in BWS studies. Third, when looking at the type of analytical method, relatively less complicated methods (e.g. best-worst counting) have become increasingly popular, especially for BWS object case. In contrast with DCE where more advanced methods (such as models that take heterogeneity into account, e.g. latent class or random parameter model) are used, ${ }^{14}$ the BWS can be analysed more easily, making the method attractive to a wide range of researchers. Details regarding the analytical methods underlying BWS can be found in Mühlbacher et al.. ${ }^{17,18}$

The quality of BWS studies in general is acceptable, but there is room for improvement as still nearly half of studies (44\%) scored either a 2 or 3 on a five-point scale. Of all studies, $35 \%$ did not include all responders in the final analyses. However, many of the 
studies that did not include all respondents did so for a specific reason (e.g. failed comprehension test or missing data). Most (90\%) BWS studies did not report whether the responders were similar to non-responders. This results in a lack of transparency that may introduce non-response bias. Future BWS studies are thus recommended to track nonresponders on some (demographic) variables and perform comparative analyses if possible. However, due to the increase popularity of online panels, this may not always be feasible. In addition, PREFS scores of " 0 " should be interpreted with caution as low PREFS scores are not synonymous with low-quality. For instance, not using all respondents for reasons of internal validity (e.g. failure of dominance test) or due to missing values may be an appropriate choice.

Our study has some potential limitations. First, as the aim of this study was to identify current practice of BWS within health care, study findings and trends may not be applicable / generalizable to BWS studies outside the health care setting. Second, as the number of BWS multi-profile case study is still limited, statements made regarding BWS multi-profile case in this manuscript should be treated with caution and are only provided to give some first insights into the conduct of BWS multi-profile case within health care. Third, we identified some discrepancies between reviewers in terms of our data collection sheet and how some fields were being interpreted (e.g. validity checks and heterogeneity). However, we resolved those discrepancies in discussions among reviewers throughout. Fourth, this study only looked at BWS as defined by Louviere et al. ${ }^{19}$ including both best and worst questions. Recently, new alternatives/variations on best-worst tasks have been published such as best-best scaling or best-only scaling. ${ }^{29-31}$ To enhance comparability and clarity, studies of this variety were not included in this review. Lastly, we only included published articles and did not search among conference proceedings.

\section{Conclusions}

The use of BWS object case and BWS profile case has drastically increased in health care, especially in the last two years. The application of BWS multi-profile case is however still very limited. In recent studies, trends toward decreasing sample sizes and number of factors in BWS object case, as well as the use of less complicated analytic methods has been observed, enhancing the usefulness of BWS method to wider health care problems and making it accessible for more researchers. Next to that, the quality of BWS studies is generally acceptable, except with regards to indicating whether the responders were similar to non-responders. Hence, this implies the need to increase transparency of BWS studies and check for non-response bias when possible. Furthermore, this study may serve as a vantage point reflecting state-of-the-art practices of BWS studies. 


\section{Acknowledgements}

KLC, BW, and $M H$ designed and planned the study. KLC and BW screened titles, abstracts, and full-texts, and produced the first draft of the manuscript. KLC, BW, IH and EJ extracted data and appraised the quality of the studies. Different versions of the manuscript were reviewed and conceptualised by all co-authors. All authors have read and approved the final manuscript. No funding has been received for the conduct of this study and/or preparation of this manuscript. 


\section{References}

1. Bridges JF, Jones C. Patient-based health technology assessment: a vision of the future. Int J Technol Assess Health Care 2007;23(01):30-5.

2. Ryan M, Gerard K. Using discrete choice experiments to value health care programmes: current practice and future research reflections. Appl Health Econ Health Policy 2003;2(1):55-64.

3. Bridges JFP, Hauber AB, Marshall D, et al. Conjoint Analysis Applications in Health-a Checklist: A Report of the ISPOR Good Research Practices for Conjoint Analysis Task Force. Value Health. 2011;14(4): 403-13.

4. Janz NK, Wren PA, Copeland LA, et al. Patient-physician concordance: preferences, perceptions, and factors influencing the breast cancer surgical decision. J Clin Oncol 2004;22(15):3091-8.

5. Facey K, Boivin A, Gracia J, et al. Patients' perspectives in health technology assessment: a route to robust evidence and fair deliberation. Int J Technol Assess Health Care 2010;26(03):334-40.

6. Bridges JF, Hauber AB, Marshall D, et al. Conjoint analysis applications in health-a checklist: a report of the ISPOR Good Research Practices for Conjoint Analysis Task Force. Value Health. 201 1; 14(4):403-13.

7. Green PE, Srinivasan V. Conjoint analysis in consumer research: issues and outlook. J Consumer Res 1978:103-23.

8. Bridges JF, Kinter ET, Kidane L, et al. Things are looking up since we started listening to patients. Patient 2008; 1(4):273-82.

9. Marley AA, Louviere JJ. Some probabilistic models of best, worst, and best-worst choices. J Mathematical Psychol 2005;49(6):464-80.

10. Finn A, Louviere JJ. Determining the appropriate response to evidence of public concern: the case of food safety. J Public Policy Marketing. 1992:12-25.

11. Szeinbach SL, Barnes JH, McGhan WF, et al. Using Conjoint Analysis to Evaluate Health State Preferences*. Drug Information Journal. 1999 July 1, 1999;33(3):849-58.

12. Mclntosh E, Louviere J. Separating weight and scale value: an exploration of best-attribute scaling in health economics. Health Economics Study Group Odense, Denmark. 2002.

13. Flynn TN, Louviere JJ, Peters TJ, et al. Best-worst scaling: what it can do for health care research and how to do it. J Health Econ 2007;26(1):171-89.

14. Clark MD, Determann D, Petrou S, et al. Discrete Choice Experiments in Health Economics: A Review of the Literature. Pharmacoeconomics. 2014;32(9):883-902.

15. de Bekker-Grob EW, Ryan M, Gerard K. Discrete choice experiments in health economics: a review of the literature. Health Econ. 2012 Feb;2 1 (2):145-72.

16. Flynn TN. Valuing citizen and patient preferences in health: recent developments in three types of bestworst scaling. Expert Rev Pharmacoecon Outcomes Res 2010;10(3):259-67.

17. Mühlbacher AC, Kaczynski A, Zweifel P, et al. Experimental measurement of preferences in health and healthcare using best-worst scaling: an overview. Health Econ Rev 2016;6(1):1-14.

18. Mühlbacher AC, Zweifel P, Kaczynski A, et al. Experimental measurement of preferences in health care using best-worst scaling (BWS): theoretical and statistical issues. Health Econ Rev 2016;6(1):1-12.

19. Louviere JJ, Flynn TN, Marley A. Best-worst scaling: Theory, methods and applications: Cambridge University Press; 2015.

20. de Bekker-Grob EW, Ryan M, Gerard K. Discrete choice experiments in health economics: a review of the literature. Health Econ 2012;21(2):145-72.

21. Joy SM, Little E, Maruthur NM, et al. Patient preferences for the treatment of type 2 diabetes: a scoping review. Pharmacoeconomics. 2013;31(10):877-92.

22. Lancsar $E$, Louviere J, Donaldson $C$, et al. Best worst discrete choice experiments in health: methods and an application. Soc Sci Med 2013;76(1):74-82.

23. Potoglou $D$, Burge $P$, Flynn $T$, et al. Best-worst scaling vs. discrete choice experiments: an empirical comparison using social care data. Soc Sci Med 201 1;72(10):1717-27.

24. Severin F, Schmidtke J, Mühlbacher A, et al. Eliciting preferences for priority setting in genetic testing: a pilot study comparing best-worst scaling and discrete-choice experiments. Eur J Hum Genet 2013; $21(11): 1202-8$. 
25. Louviere JJ, Islam T. A comparison of importance weights and willingness-to-pay measures derived from choice-based conjoint, constant sum scales and best-worst scaling. Journal of Business Research. 2008;61(9):903-11.

26. Gallego G, Dew A, Lincoln M, et al. Should I stay or should I go? Exploring the job preferences of allied health professionals working with people with disability in rural Australia. Human Resources for Health. $2015 ; 13(1): 53$.

27. Lancsar E, Louviere J, Donaldson C, et al. Best worst discrete choice experiments in health: Methods and an application. Social science \& medicine. 2013;76:74-82.

28. de Bekker-Grob EW, Donkers B, Jonker MF, et al. Sample size requirements for discrete-choice experiments in healthcare: a practical guide. Patient 2015;8(5):373-84.

29. Ghijben P, Lancsar E, Zavarsek S. Preferences for oral anticoagulants in atrial fibrillation: a best-best discrete choice experiment. Pharmacoeconomics. 2014;32(11):1115-27.

30. Meyfroidt S, Hulscher M, De Cock D, et al. A maximum difference scaling survey of barriers to intensive combination treatment strategies with glucocorticoids in early rheumatoid arthritis. Clin Rheumatol 2015;34(5):861-9.

31. Van Hulst L, Kievit W, Van Bommel R, et al. Rheumatoid arthritis patients and rheumatologists approach the decision to escalate care differently: results of a maximum difference scaling experiment. Arthritis Care Res. $2011 ; 63(10): 1407-14$

32. Louviere JJ, Flynn TN. Using best-worst scaling choice experiments to measure public perceptions and preferences for healthcare reform in australia. Patient 2010;3(4):275-83.

33. Kurkjian TJ, Kenkel JM, Sykes JM, et al. Impact of the current economy on facial aesthetic surgery. Aesthet Surg J 2011 Sep;31(7):770-4

34. Gallego G, Bridges JFP, Flynn T, et al. Using best-worst scaling in horizon scanning for hepatocellular carcinoma technologies. Int J Technol Assess Health Care 2012;28(3):339-46.

35. Marti J. A best-worst scaling survey of adolescents' level of concern for health and non-health consequences of smoking. Soc Sci Med 2012;75(1):87-97.

36. Mazanov J, Huybers T, Connor J. Prioritising health in anti-doping: What Australians think. J Sci Med Sport. $2012 ; 15(5): 381-5$

37. Silverman S, Calderon A, Kaw K, et al. Patient weighting of osteoporosis medication attributes across racial and ethnic groups: A study of osteoporosis medication preferences using conjoint analysis. Osteoporosis Int 2013;24(7):2067-77.

38. Cozmuta R, Merkel PA, Wahl E, et al. Variability of the impact of adverse events on physicians' decision making. BMC Med Inform Decis Mak 2014;14:86.

39. Ejaz A, Spolverato G, Bridges JF, et al. Choosing a Cancer Surgeon: Analyzing Factors in Patient Decision Making Using a Best-Worst Scaling Methodology. Ann Surg Oncol 2014;21(12):3732-8.

40. Hauber $A B$, Mohamed $A F$, Johnson FR, et al. Understanding the relative importance of preserving functional abilities in Alzheimer's disease in the United States and Germany. Qual Life Res 2014;23(6):1813-21.

41. Hofstede SN, van Bodegom-Vos L, Wentink MM, et al. Most important factors for the implementation of shared decision making in sciatica care: ranking among professionals and patients. PloS One 2014;9(4):e94176.

42. Torbica A, De Allegri $M$, Belemsaga $D$, et al. What criteria guide national entrepreneurs' policy decisions on user fee removal for maternal health care services? Use of a best-worst scaling choice experiment in west africa. J Health Serv Res Policy. 2014;19(4):208-15.

43. van Til J, Groothuis-Oudshoorn C, Lieferink M, et al. Does technique matter; a pilot study exploring weighting techniques for a multi-criteria decision support framework. Cost Eff Resource Alloc 2014:12-22.

44. Yuan Z, Levitan B, Burton P, et al. Relative importance of benefits and risks associated with antithrombotic therapies for acute coronary syndrome: Patient and physician perspectives. Curr Med Res Opin $2014 ; 30(9): 1733-41$

45. Beusterien K, Kennelly MJ, Bridges JF, et al. Use of best-worst scaling to assess patient perceptions of treatments for refractory overactive bladder. Neurol Urodyn 2016;35(8):1028-1033.

46. Fraenkel L, Lim J, Garcia-Tsao G, et al. Variation in Treatment Priorities for Chronic Hepatitis C: A Laten Class Analysis. Patient 2015:1-9.

47. Hashim $\mathrm{H}$, Beusterien K, Bridges JF, et al. Patient preferences for treating refractory overactive bladder in the UK. Int Urol Nephrol 2015;47(10):1619-27. 
48. Malhotra C, Chan N, Zhou J, et al. Variation in physician recommendations, knowledge and perceived roles regarding provision of end-of-life care. BMC Palliat Care. 2015:14-52.

49. Narurkar V, Shamban A, Sissins $P$, et al. Facial treatment preferences in aesthetically aware women. Dermatologic surgery : official publication for American Society for Dermatologic Surgery [et al]. 201501 Apr;41:S153-S60.

50. Peay HL, Hollin I, Bridges J. Prioritizing parental worry associated with duchenne muscular dystrophy using best-worst scaling. J Genet Couns. 2016;25(2):305-13.

51. Ross $\mathrm{M}$, Bridges JFP, $\mathrm{Ng} \mathrm{X}$, et al. A best-worst scaling experiment to prioritize caregiver concerns about ADHD medication for children. Psychiatr Serv 2015;66(2):208-11

52. Wittenberg $E$, Bharel M, Saada A, et al. Measuring the preferences of homeless women for cervical cancer screening interventions: Development of a best-worst scaling survey. Patient 2015;8(5):455-67

53. Yan K, Bridges JF, Augustin $\mathrm{S}$, et al. Factors impacting physicians' decisions to prevent variceal hemorrhage. BMC Gastroenterol 2015;15:55.

54. Yu T, Holbrook JT, Thorne JE, et al. Outcome preferences in patients with noninfectious uveitis: Results of a best-worst scaling studypatient outcome preferences in noninfectious uveitis. Investigat Ophthalmol Vis Sci 2015;56(11):6864-72

55. Coast J, Flynn TN, Salisbury C, et al. Maximising responses to discrete choice experiments: A randomised trial. Appl Health Econ Health Policy. 2006;5(4):249-60

56. Coast J, Flynn TN, Natarajan L, et al. Valuing the ICECAP capability index for older people. Soc Sci Med 2008;67(5):874-82.

57. Flynn TN, Louviere JJ, Peters TJ, et al. Estimating preferences for a dermatology consultation using BestWorst Scaling: Comparison of various methods of analysis. BMC Med Res Methodol 2008;8(76).

58. Al-Janabi H, Flynn TN, Coast J. Estimation of a preference-based carer experience scale. Med Decis Making. $20112011 ; 31(3): 458-68$.

59. Potoglou D, Burge P, Flynn T, et al. Best-worst scaling vs. discrete choice experiments: An empirical comparison using social care data. Soc Sci Med 201 1;72(10):1717-27.

60. Ratcliffe J, Couzner L, Flynn T, et al. Valuing child health utility 9D health states with a young adolescent sample: A feasibility study to compare best-worst scaling discrete-choice experiment, standard gamble and time trade-off methods. Appl Health Econ Health Policy. 2011 ;9(1):15-27.

61. Knox SA, Viney RC, Street DJ, et al. What's good and bad about contraceptive products?: a best-worst attribute experiment comparing the values of women consumers and GPs. Pharmacoeconomics. 2012;30(12):1187-202.

62. Molassiotis A, Emsley R, Ashcroft D, et al. Applying Best-Worst scaling methodology to establish delivery preferences of a symptom supportive care intervention in patients with lung cancer. Lung Cancer $2012 ; 77(1): 199-204$.

63. Najafzadeh M, Lynd LD, Davis JC, et al. Barriers to integrating personalized medicine into clinical practice: A best-worst scaling choice experiment. Genet Med 2012 May; 14(5):520-6.

64. Netten A, Burge P, Malley J, et al. Outcomes of social care for adults: developing a preference-weighted measure. Health Technol Assess 2012;16(16):1-166.

65. Ratcliffe J, Flynn T, Terlich F, et al. Developing adolescent-specific health state values for economic evaluation: An application of profile case best-worst scaling to the child health utility 9D. PharmacoEconomics. 2012;30(8):713-27.

66. Severin F, Schmidtke J, Muhlbacher A, et al. Eliciting preferences for priority setting in genetic testing: A pilot study comparing best-worst scaling and discrete-choice experiments. Eur J Hum Genet 2013;21(11): 1202-8.

67. Yoo HI, Doiron D. The use of alternative preference elicitation methods in complex discrete choice experiments. J Health Econ 2013;32(6):1 166-79.

68. Damery S, Biswas $M$, Billingham $L$, et al. Patient preferences for clinical follow-up after primary treatment for soft tissue sarcoma: A cross-sectional survey and discrete choice experiment. Eur J Surg Oncol 2014; 40(12):1655-61.

69. Hollin IL, Peay HL, Bridges JF. Caregiver preferences for emerging duchenne muscular dystrophy treatments: a comparison of best-worst scaling and conjoint analysis. Patient 2015;8(1):19-27.

70. Peay HL, Hollin I, Fischer R, et al. A community-engaged approach to quantifying caregiver preferences for the benefits and risks of emerging therapies for duchenne muscular dystrophy. Clin Ther 2014;36(5): 624-37. 
71. Ratcliffe J. A think aloud study comparing the validity and acceptability of discrete choice and best worst scaling methods. PloS One. 2014;9(4):e90635.

72. Ungar WJ, Hadioonzadeh A, Najafzadeh $M$, et al. Quantifying preferences for asthma control in parents and adolescents using best-worst scaling. Respir Med 2014;108(6):842-51.

73. Whitty JA, Ratcliffe J, Chen G, et al. Australian Public Preferences for the Funding of New Health Technologies: A Comparison of Discrete Choice and Profile Case Best-Worst Scaling Methods. Med Decis Making 2014 Apr 8;34(5):638-54.

74. dosReis $\mathrm{S}, \mathrm{Ng} \mathrm{X}$, Frosch $\mathrm{E}$, et al. Using best-worst scaling to measure caregiver preferences for managing their child's ADHD: A pilot study. Patient. 2015;8(5):423-31.

75. Flynn TN, Huynh E, Peters TJ, et al. Scoring the Icecap-a capability instrument. Estimation of a UK general population tariff. Health Econ 2015;24(3):258-69.

76. Franco MR, Howard K, Sherrington $C$, et al. Eliciting older people's preferences for exercise programs: a best-worst scaling choice experiment. J Physiother 2015;61(1):34-41

77. Gendall P, Hoek J, Taylor R, et al. Should support for obesity interventions or perceptions of their perceived effectiveness shape policy? Aust N Z J Public Health. 2015;39(2):172-6.

78. Jones LG, Hawkins GE, Brown SD. Using best-worst scaling to improve psychological service delivery: an innovative tool for psychologists in organized care settings. Psychol Serv 2015; 12(1):20-7.

79. O'Hara NN, Roy L, O'Hara LM, et al. Healthcare Worker Preferences for Active Tuberculosis Case Finding Programs in South Africa: A Best-Worst Scaling Choice Experiment. PloS One. 2015;10(7):e0133304.

80. Ratcliffe J, Huynh E, Stevens K, et al. Nothing about us without us? A comparison of adolescent and adult health-state values for the child health utility-9D using profile case best-worst Scaling. Health Econ $2016 ; 25(4): 486-96$

81. Tsao NW, Khakban A, Gastonguay L, et al. Perceptions of British Columbia residents and their willingness to pay for medication management services provided by pharmacists. Can Pharm J 2015; 148(5):263-73.

82. Brown TM, Pashos CL, Joshi AV, et al. The perspective of patients with haemophilia with inhibitors and their care givers: preferences for treatment characteristics. Haemophilia $2011 ; 17(3): 476-82$

83. Hoek J, Wong C, Gendall P, et al. Effects of dissuasive packaging on young adult smokers. Tob Control $2011 ; 20(3): 183-8$.

84. Cameron MP, Newman PA, Roungprakhon S, et al. The marginal willingness-to-pay for attributes of a hypothetical HIV vaccine. Vaccine 2013;31(36):3712-7.

85. Maubach N, Hoek J, Mather D. Interpretive front-of-pack nutrition labels. Comparing competing recommendations. Appetite. 2014;82:67-77.

86. Xie F, Pullenayegum E, Gaebel K, et al. Eliciting preferences to the EQ-5D-5L health states: discrete choice experiment or multiprofile case of best-worst scaling? Eur J Health Econ 2014;15(3):281-8. 


\section{Supplemental material S5.1}

Pubmed search: (best-worst scaling[Text Word] OR best worst scaling[Text Word] OR best worst [Text Word] OR maxdiff[Text Word] OR max diff[Text Word] OR maxdiff scaling[Text Word] OR max diff scaling[Text Word] OR Maximum difference scaling[Text Word] OR object scaling [Text Word]) AND (("1990/01/01"[PDat] : "2016/04/06"[PDat]) AND English[lang])

\section{EMBASE search}

1 Best-worst scaling.tw.

2 Best worst scaling.tw.

3 Best worst

4 Maxdiff.tw.

5 Max Diff.tw.

6 Maxdiff scaling.tw.

7 Max diff scaling.tw.

8 Maximum difference scaling.tw.

9 Object scaling.tw.

10 Object-scaling.tw.

111 or 2 or 3 or 4 or 5 or 6 or 7 or 8 or 9 or 10

12 Limit 11 to (English language and $y r=" 1990$-Current") 

Economic evaluations within epilepsy
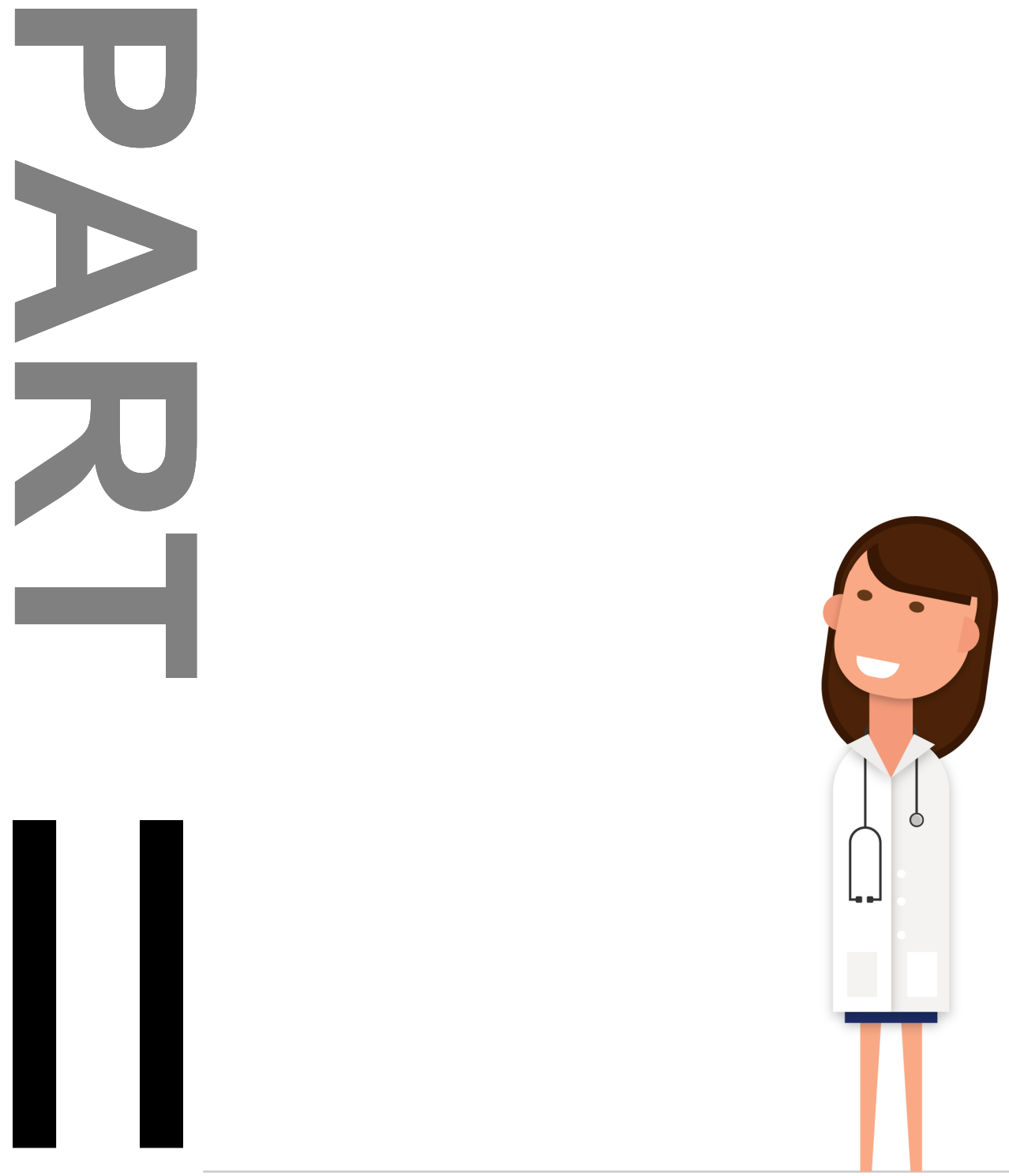



\section{How to prepare a}

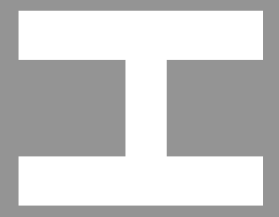

systematic review of

economic evaluations for

Informing evidence-based

healthcare decisions

Data extraction, risk of bias, and

transferability (Part 3/3)
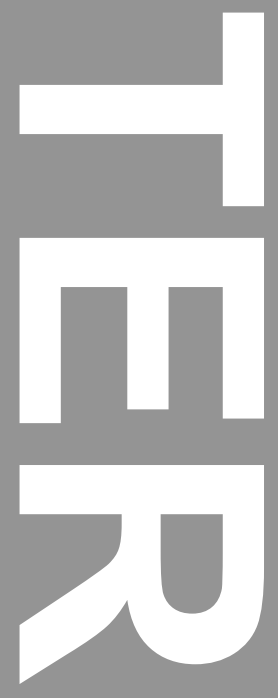

B.F.M. Wijnen G.A.P.G. van Mastrigt

W.K. Redekop H.J.M. Majoie

R.J.A. de Kinderen

S.M.A.A. Evers

Expert Rev Pharmacoecon Outcomes Res, 2016; 16(6):723-732 


\section{Summary}

This article is part of the series "How to Prepare a Systematic Review (SR) of Economic Evaluations (EE) for Informing Evidence-based Healthcare Decisions" in which a five-stepapproach for conducting a SR of EE is proposed. This paper explains the data extraction process, the risk of bias assessment and the transferability of EEs by means of a narrative review and expert opinion.
SRs play a critical role in determining the comparative cost-effectiveness of healthcare interventions. It is important to determine the risk of bias and the transferability of an EE. Over the past decade, several criteria lists have been developed. This article aims to provide recommendations on these criteria lists based on the thoroughness of development, feasibility, overall quality, recommendations of leading organizations, and widespread use. 


\section{Introduction}

In this article, which focuses on Step 3 of the overall framework (see Figure $6.1{ }^{1}{ }^{1}$ ), attention will be paid to data extraction, risk of bias assessment, and transferability when preparing a systematic literature review of economic evaluations (SR-EE). Moreover, the article is also perfectly readable as a stand-alone read.

\section{Step 1: Initiating a SR of EEs; described in van Mastrigt et al. 2016}

Step 2: Identifying full EEs; described in Thielen et al. 2016

\section{Step 3: Data extraction, risk of bias, and transferability}

Figure 6.1 Overview of 5-step approach for preparing a systematic review of economic evaluations of healthcare interventions.

In 2003, the AGREE (Appraisal of Guidelines for Research and Evaluation) collaboration issued an instrument for evaluating the process of developing clinical practice guidelines (CPGs) and the quality of reporting. ${ }^{2}$ Updated in 2010 to AGREE II, ${ }^{3}$ this instrument consists of several domains, of which "rigor of development", and "applicability" are of importance to this paper. These two domains reveal that recommendations need to consider (a) health benefits, side effects and risks (domain 3, item 11), as well as (b) the potential resource implications of applying the recommendations or, in other words, 'what does it cost when a certain recommendation is implemented'? (domain 5, item 20). ${ }^{4}$ This means that CPGs need to consider not only the potential effectiveness of healthcare interventions but also their cost-effectiveness, as well as their overall impact on budget.

When creating or updating CPGs, SRs play a critical role in determining the comparative cost-effectiveness of healthcare interventions, with the goal of creating an efficient healthcare system. ${ }^{5}$ However, EEs are prone to several biases. Bias occurs when there is a difference between the true value (in the population) and the observed value (in the study) from any cause other than sampling variability. ${ }^{6} \mathrm{~A}$ bias can be unintentional or 
intentional and can have either substantial or little impact on the results of an EE. ${ }^{7}$ In order to make optimum policy decisions it is important to determine the risk of bias in an EE. ${ }^{7}$ For example, EEs may have a perspective that is too narrow, or may fail to incorporate important costs. In addition, one should be aware of the opportunity costs from decisions based on poor quality EEs (i.e. misleading study findings, lack of transparency and clarity in reporting ${ }^{8}$ ). Hence, in the past decades, several criteria lists have been developed to assess the risk of bias in EEs and to evaluate the transferability of EE. These lists are important tools that help to interpret and compare individual studies. However, due to the number and availability of all these tools, it can be difficult to make a careful selection.

Full EEs can differ in a variety of aspects and all aspects can affect the quality of the evaluation and consequently bias results. The term "full EE" refers to the comparative analysis of alternative courses of action in terms of both costs (resource use) and consequences (outcomes, effects). ${ }^{5}$ Basically, there are two approaches to performing an EE study ${ }^{7}$ : 1) an EE which is piggy-backed onto a clinical effectiveness study (e.g. a randomized controlled trial or observational study), often called a trial-based EE; and 2) a model-based EE, in which data from a wide range of sources (randomized controlled trials, observational studies, trial-based EEs, other literature or reports etc.) are combined using an economic model. Both are complementary to each other. ${ }^{5}$ For a model-based EE, it is important that the external validation of the results, the key structural assumptions, and the data sources and derivation of the input data used in the model are well described. This way, potential policymakers or CPG developers are able to incorporate the strengths and limitations of the EE in their evaluation of the evidence. ${ }^{9}$ It is important to keep in mind that the quality of EEs can be only as good as the quality of the trials on which they are based. ${ }^{9}$ This holds true for both model-based EE and trial-based EE. As the field of effectiveness studies is relatively old in comparison with the field of EEs, methodological issues (i.e. the Cochrane Collaboration's tool for assessing the risk of bias ${ }^{10}$ ), reporting standards (i.e. the CONSORT statement ${ }^{11}$ and the STROBE statement ${ }^{12}$ ) and the grading of evidence methods (i.e. the GRADE method for determining the quality of a body of evidence ${ }^{13}$ ) have been established and are regularly being used and recognized in the development of CPGs for SRs. For example, as in clinical studies, biases in (full) EEs can occur as a result of poor methodological quality, which can impact the validity of the results in terms of generalizability or transferability. ${ }^{7}$ Evers et al. ${ }^{7}$ have identified some methodological biases (so-called pre-trial and during trial biases), e.g. biases which occur as a result of a narrow perspective, inefficient comparator, cost measurement omission, or inappropriate discounting. In addition, they have identified some after-trial biases such as reporting and dissemination bias. Although these biases are thought of more in relation to trial-based $\mathrm{EE}$, most of them are also applicable to model-based EE.

Therefore, this article will focus only on risk of bias and transferability checklists specifically tailored for the critical appraisal of EEs. SR-EEs can be categorized roughly into three groups; 1) multi-purpose reviews, 2) reviews for informing the development of clinical practice guidelines (CPGs), and 3) reviews for developing decision analytic models. Both multi-purpose SR-EEs and SR-EEs for guideline development aim to 
synthesize and critically appraise existing EEs of a healthcare intervention or disease area in order to inform policy decisions. ${ }^{1,14}$ The guidance in this paper covers only the first two types of SR categories. Accordingly, this article is aimed mainly at CPG developers, although the checklists can also aid others who want to prepare SR-EEs, like HTA researchers, systematic reviewers and students, as they seek to identify the different steps, important key sources and practical information to gain basic knowledge on this topic.

To be sure all relevant data of the included studies has been collected, it is important to develop a data extraction sheet for more systematic data collection. A data extraction sheet is an organized table in which all relevant items which need to be extracted for the review are listed; this needs to be completed for every study in order to collect data systematically. The inclusion of items depends on the research question or study objective, and on the study design and outcomes predefined in the study protocol (see Step 1.3 of the overall framework in Van Mastrigt et al. ').

Accordingly, this article will first discuss the data extraction sheet, and then present an overview of the methods most commonly used to assess the risk of bias and the transferability of EEs.

\section{Step 3.1 of the overall framework: Data extraction}

This step entails extracting all relevant data from the included studies. For every SR-EE a tailored data extraction sheet needs to be developed. Which items are included depends on the research question or study objective, and on the study design and outcomes predefined in the study protocol (see Step 1: "Initiating a SR-EEs" of the overall framework). Consideration of the care pathway can be helpful in structuring the data extraction. ${ }^{15}$ In addition, the risk of bias in the included studies needs to be appraised, in order to assess the possible impact of bias on the results of SRs (Step 3.2). Excel (Microsoft Office) can be used for the digital registration of items. It is highly recommended that the extraction sheet be piloted for user-friendliness and completeness, using a few sample studies. ${ }^{15,16}$ Then, if needed, the data extraction form can be adapted before starting data extraction of all studies. For Step 3.1, the data extraction of study characteristics, methods and outcomes, it is important to simply report the findings as reported by the authors of the study and not draw any conclusions from them. This is in contrast with step 3.2, the risk of bias assessment, in which a critical appraisal of the studies is necessary for answering all questions.

There are several example data extraction forms available from the literature, ${ }^{15-17}$ containing many common items. These items can be classified in two groups. First, the general study characteristics: these are, for instance, author, year of publication, type of intervention, control treatment, eligibility criteria, study perspective, type of EE and analytic approach (trial-based versus model-based). Second are the study methods and outcomes: these include resource use, costs, effects, measurement, valuation methods, incremental cost-effectiveness ratios, uncertainty analyses, sensitivity analysis and conclusions. Based 
on our experience we recommend including all relevant items from the list in Table 6.1 in the initial data extraction. If one is particularly interested in model-based EEs one could extend this list with the external validation of the results, the key structural assumptions, and data sources and derivation of the input data used in the model. Using a picklist is recommended for choosing the different answers. Furthermore, in order to facilitate interpretation of the results, a disaggregated presentation of the results, as well as incremental cost-effectiveness ratios, are highly recommended. ${ }^{15}$ When presenting information derived from the data extraction, in some cases it may be more appealing to present in a table than others. Table 6.2 and Table 6.3 provide an example of how to present the general study characteristics and the economic evidence. In this study of De Kinderen et al. ${ }^{18}$ a ketogenic diet (KD) is compared with care as usual to reduce epileptic seizures in children with intractable epilepsy.

Table 6.1 Items and explanation for the data extraction of economic evidence

\begin{tabular}{|c|c|c|}
\hline \multicolumn{2}{|c|}{ Number Type of items } & \multirow{2}{*}{$\begin{array}{l}\text { Explanation } \\
\text { State the name of person who has filled out the data extraction } \\
\text { sheet. }\end{array}$} \\
\hline & Checklist completed by & \\
\hline & General study characteristics & \\
\hline 1 & First author and year of publication & $\begin{array}{l}\text { Report: First author, title, journal name, publication date, } \\
\text { volume, issue, page numbers and link to the publication. }\end{array}$ \\
\hline \multirow[t]{4}{*}{2} & Sources of funding & Report: The source of funding cited in the paper: Write "Stated" \\
\hline & & $\begin{array}{l}\text { or "Not Stated" and specify. If any, give name(s) of } \\
\text { organization or corporation. Specify if possible the source type }\end{array}$ \\
\hline & & (public research funds, NGO, government, \\
\hline & & academic/university, healthcare industry or other). \\
\hline 3 & Competing interests & $\begin{array}{l}\text { Competing interests: write "stated" or "not stated" and specify } \\
\text { if any. }\end{array}$ \\
\hline 4 & Publication type & Describe type of publication ** (Journal paper, HTA report) \\
\hline 5 & Setting & $\begin{array}{l}\text { List the country/countries, setting and or locations for economic } \\
\text { evaluation. }\end{array}$ \\
\hline 6 & Patient characteristics & $\begin{array}{l}\text { Summarize inclusion and exclusion criteria (eligibility } \\
\text { criteria/demographics). }\end{array}$ \\
\hline 7 & Type of intervention & Describe the experimental treatment (service, program). \\
\hline 8 & Control treatment & Describe the control treatment (service, program). \\
\hline 9 & Eligibility criteria & $\begin{array}{l}\text { Describe the eligible population and the population used for } \\
\text { effect/cost data. }\end{array}$ \\
\hline 10 & Study perspective & $\begin{array}{l}\text { State the viewpoint of the analysis: (society, healthcare, insurer, } \\
\text { care provider, patient and family). }\end{array}$ \\
\hline 11 & Type of EEs $* * *$ & $\begin{array}{l}\text { Specify the form of economic evaluation being used (e.g. CEA, } \\
\text { CUA, CBA, CCA). }\end{array}$ \\
\hline \multirow[t]{2}{*}{12} & Analytic approach & Describe the analytic approach: trial-based or model-based. \\
\hline & $\begin{array}{l}\text { Methods and outcomes of economic } \\
\text { evaluations }\end{array}$ & \\
\hline 13 & $\begin{array}{l}\text { Time frame of the analysis (time } \\
\text { horizon) }\end{array}$ & State the time horizon for both costs and benefits. \\
\hline 14 & Discount rate & Was discounting performed? \\
\hline 15 & Discount rate for costs & What was the discount rate for the costs $(\mathrm{s})$ ? \\
\hline 16 & Discount rate for effects & $\begin{array}{l}\text { What was the discount rate for the effects(s)? (i.e. the rate used } \\
\text { to account for different timing of costs and effects) }\end{array}$ \\
\hline
\end{tabular}


Table 6.1 (continued)

\begin{tabular}{|c|c|c|}
\hline \multicolumn{2}{|c|}{ Number Type of items } & \multirow{2}{*}{$\begin{array}{l}\text { Explanation } \\
\text { State the name of person who has filled out the data extraction } \\
\text { sheet. }\end{array}$} \\
\hline & Checklist completed by & \\
\hline 17 & Inflation rate & $\begin{array}{l}\text { Was adjustment for inflation performed if unit costs stemmed } \\
\text { from different years? }\end{array}$ \\
\hline 18 & Reference year & What was the reference year of the analysis? \\
\hline 19 & If model-based & $\begin{array}{l}\text { Detail any model used (Markov, Decision Tree, Discrete Event } \\
\text { Simulation etc.) }{ }^{* * *}\end{array}$ \\
\hline 20 & Type and category of costs & $\begin{array}{l}\text { Describe the different cost types and categories used (e.g. } \\
\text { direct in healthcare, indirect healthcare, intangible costs). }\end{array}$ \\
\hline 21 & Data source of resource use & $\begin{array}{l}\text { Describe the data for resource use (e.g. , clinical trials, } \\
\text { administrative data, clinical databases, medical records, } \\
\text { published literature). }\end{array}$ \\
\hline \multirow[t]{2}{*}{22} & Methods for identifying resource use & $\begin{array}{l}\text { Describe the methods used to identify resource use } \\
\text { (questionnaire, survey, cost dairies, expert consultation, formal } \\
\text { consensus methods). }\end{array}$ \\
\hline & $\begin{array}{l}\text { Assumptions of the measurement of } \\
\text { resources }\end{array}$ & $\begin{array}{l}\text { Describe, for instance, method of imputation when incomplete } \\
\text { measurement occurred. }\end{array}$ \\
\hline 22 & $\begin{array}{l}\text { Costs (in reported currency or in } \\
\text { converted currency) }\end{array}$ & $\begin{array}{l}\text { Present relevant costs and outcomes in both disaggregated and } \\
\text { aggregated form (with confidence intervals, measures of } \\
\text { significance). }\end{array}$ \\
\hline 24 & Methods of used to calculate unit costs & $\begin{array}{l}\text { Describe the methods used to identify relevant unit costs } \\
\text { (guidelines, own cost price calculations, literature). }\end{array}$ \\
\hline 25 & Costs* & $\begin{array}{l}\text { Present relevant costs in disaggregated and aggregated form } \\
\text { (with confidence intervals, measures of significance). }\end{array}$ \\
\hline 27 & Data source of effects & $\begin{array}{l}\text { Specify where utilities or benefits came from (literature values, } \\
\text { elicited in the study etc.). }\end{array}$ \\
\hline 28 & Methods of measurement of effects & $\begin{array}{l}\text { Specify source of effectiveness estimates (stated WTP, revealed } \\
\text { WTP, conjoint analysis etc.). }\end{array}$ \\
\hline 29 & Methods of valuation of effects & $\begin{array}{l}\text { Specify methods of valuation of effects (indirect or direct } \\
\text { measurement). }\end{array}$ \\
\hline 30 & Effects * & $\begin{array}{l}\text { Present relevant effects (utilities, (health) benefits and } \\
\text { outcomes) in disaggregated and aggregated form (with } \\
\text { confidence intervals, measures of significance) }\end{array}$ \\
\hline 31 & Incremental cost-effectiveness ratios & $\begin{array}{l}\text { State the summary measure of benefit for CUA (eg. QALY or } \\
\text { DALY etc.) }\end{array}$ \\
\hline 32 & $\begin{array}{l}\text { Analyses of uncertainty (e.g. sensitivity } \\
\text { analyses) }\end{array}$ & $\begin{array}{l}\text { Describe the analyses of uncertainty (e.g. statistical } \\
\text { comparison, bootstrapping, sensitivity analysis (one way, muli- } \\
\text { way, threshold analysis, analysis of extremes, best/worst case } \\
\text { analysis) and probabilistic sensitivity analysis. }\end{array}$ \\
\hline 34 & $\begin{array}{l}\text { Outcome(s) of analyses of sensitivity } \\
\text { analyses }\end{array}$ & \\
\hline 35 & Authors' conclusions & Report the conclusions of the authors \\
\hline
\end{tabular}

*When reporting the study outcomes it is preferred to report the degree of uncertainty; therefore, in addition to reporting the mean (or median), a standard deviation (or range) should be reported. ${ }^{5}$ * duplicate publications of the same study need to be linked together. ${ }^{* *}$ ( $C E A=$ Cost-effectiveness analyses, CUA=Cost-utility analyses, $\mathrm{CCA}=$ cost-consequence analyses, $\mathrm{CBA}=$ cost benefit analyses. ${ }^{* * *}$ Check for details: Philips et al. ${ }^{18}$ 


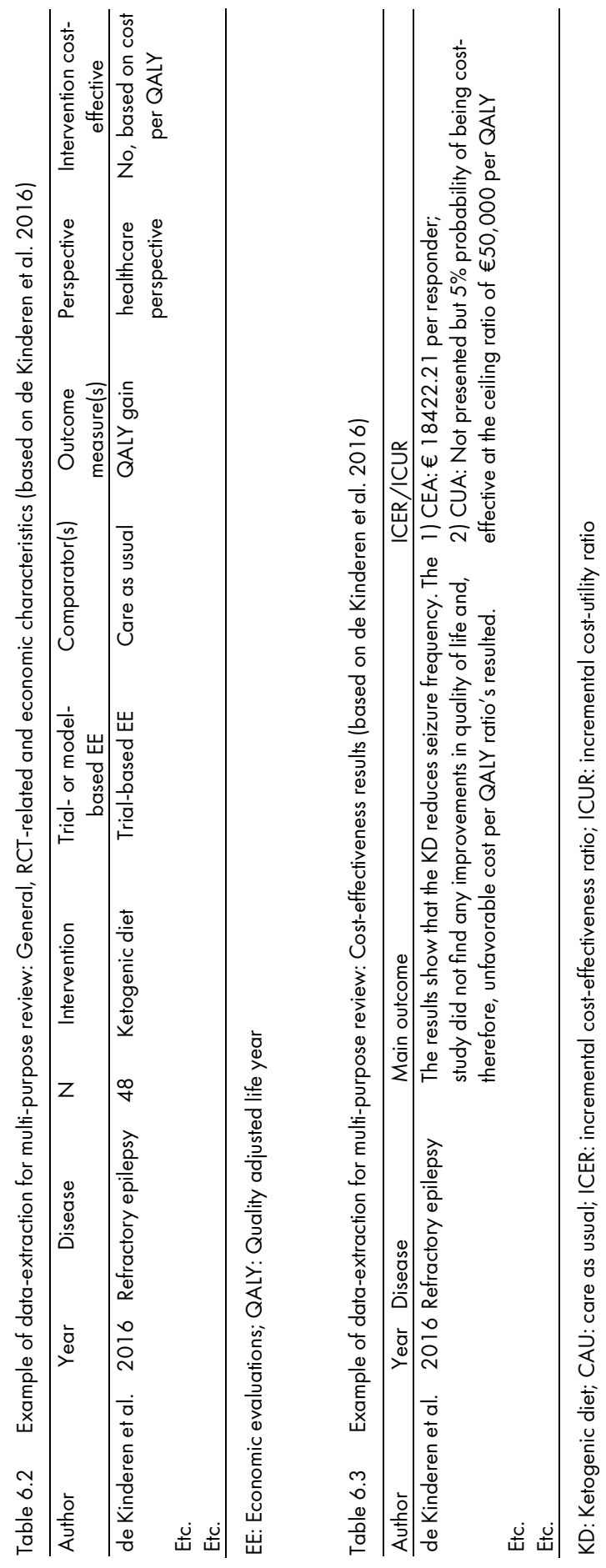




\section{Step 3.2 of the overall framework: Risk of bias assessment}

This step focuses on the risk of bias assessment for the studies included in a SR-EEs. Although the risk of bias in EEs is equally important in CPG development and multi-purpose reviews, differences might occur in the type of EEs included. In general, full EEs are recommended as being the most valid way to conduct an EE. Accordingly, we would like to stress that full EEs should be preferred over partial EEs at all times. However, in CPG development, and/or in the absence of full EEs, one might be interested in partial EEs (e.g. costs analyses). Partial EEs may represent important intermediate stages in our understanding of the costs and consequences of health services programs and therefore might be convenient, e.g. in (early) CPG development. ${ }^{5}$ Hence, both full and partial EEs will be discussed separately, with the difference that partial EEs will be discussed only in relation to CPG development.

In addition, although the risk of bias assessment and the way of reporting the results of EEs might seem like two distinct topics, in practice both topics are intertwined and difficult to differentiate from one another. For example, in order for a flawlessly conducted EE to be perceived as having "low risk of bias", it should be reported in a transparent and comprehensive way. While this article will focus on the risk of bias assessment of EEs, it is important to keep this in mind when reading the rest of the article. Specifically in order to assess the reporting quality of an EE, the ISPOR taskforce has developed the Consolidated Health Economic Evaluation Reporting Standards" (CHEERS), in which recommendations are made to optimize the reporting of health EEs for all types of EE derived by either trialor model-based EE. ${ }^{8}$

\section{Risk of bias assessment for multipurpose SR-EEs}

As full EEs are considered to be the best strategy to answer efficiency questions, ${ }^{5}$ most checklists focus solely on full EEs. Over the past decades, several criteria checklists for the risk of bias assessment of full EEs have been constructed. A recent SR by Walker et al. ${ }^{19}$ identified 10 checklists and criteria lists published between 1992 and 2011 . In addition to the studies identified by Walker et al., we identified three additional studies: the checklists of Sculpher et al., ${ }^{20}$ Philips et al., ${ }^{21}$ and Caro et al. ${ }^{22}$ (ISPOR checklist). An assessment of these checklists was made based on the purpose of the development, thoroughness of the development process, number of criteria checklists, operationalization of the questions, assessment instructions, time to complete, whether the checklist includes an overall quality score, and the number of references (providing us with an indication of its frequency of use). The full overview can be found in online supplementary material I. The BMJ checklist $^{23}$ and the CHEC-extended checklist, which is an extension of the original CHEC checklist to include a question regarding model-based $\mathrm{EE},{ }^{24-26}$ are commonly considered to have more scrutiny than most other lists. ${ }^{16}$ Accordingly, the Cochrane Collaboration recommends using one of these two checklists to assess the risk of bias of full trial-based EEs conducted alongside single effectiveness studies. In addition, the BMJ checklist is also 
recommended by the Campbell \& Cochrane Economics Methods Group for use in SRs. However, if the scope of the critical review of EEs encompasses relevant economic modelling studies, then assessments of the risk of bias of such studies will need to be informed by a different checklist, as the BMJ checklist and the CHEC-extended checklist are relevant but not sufficient for modelling studies. ${ }^{16}$ Both the Cochrane Collaboration and the National Institute for Health and Care Excellence (NICE) recommend using the Philips checklist to assess modelling studies. ${ }^{27}$ However, as the Philips checklist contains a relatively large amount of criteria, using this checklist may not be feasible if one is interested specifically in a large number of model-based EEs. In cases where one is specifically interested in model-based EE and if the expected number of included studies is low (e.g. $<10$ studies; pragmatic decision) the Philips checklist ${ }^{21}$ could be used. In cases where the number of included model-based EEs is high (e.g. >10 studies; pragmatic decision), considering the feasibility and thoroughness of the developmental process, the ISPOR checklist is likely to be more practical for reviewing purposes. ${ }^{22}$

In Table 6.4 an example is provided of how one can conduct the appraisal of a study using the $\mathrm{CHEC}$-extended checklist. The appraisal process in this example was guided by assessment instructions specifically designed for the CHEC-checklist. Again, the study of De Kinderen et al. ${ }^{18}$ was used as an example. For most checklists, such instructions are available and make the appraisal process more straightforward.

\section{Risk of bias assessment in SR-EEs for CPG development}

The Grading of Recommendations Assessment, Development, and Evaluation (GRADE) approach has been developed to rate the confidence in effect estimates (quality of evidence) for clinical outcomes and is often used and highly recommended in CPG development. ${ }^{13}$ This approach was recently extended to include the quality of economic evidence (both for partial and full EEs). In general, the GRADE recommends that important differences in resource use should be included along with other important outcomes in the evidence profiles and summary of findings tables. In this process, four key steps have been identified: 1) identify items of resource use that may differ among alternative management strategies and that are potentially important to patients and decision makers; 2) find evidence for the differences in resource use between the options being compared; 3) rate the confidence in estimates of effect; and 4) if the evidence profile and summary of findings tables are being developed to inform recommendations in a specific setting, value the resource use in terms of costs for the specific setting for which recommendations are being made. ${ }^{28}$ Resource use and the cost of treatments are included in the summary of findings tables. The cost-effectiveness estimates are included in the evidence profiles as background information. In this way it is possible to include the results of partial EEs (e.g. cost analyses) in a systematic way in the GRADE approach when developing guidelines. However, the GRADE recommends excluding model-based EE as they are often based on trials which could lead to double counting. ${ }^{28}$ 
Table 6.4 Example of critical appraisal of the quality of the economic evaluation using the CHEC-checklist (based on de Kinderen et al. 2016).

\footnotetext{
CHEC-extended items (Evers et al. 2005; De Kinderen et al. (2015) Odnoletkova et al. 2014)

1 Is the study population clearly described? $\quad$ Yes (see in method section Study population and sample size and result section; Table 2)

2 Are competing alternatives clearly described? Yes, sufficient details provided on usual care group vs. ketogenic diet group.

3 Is a well-defined research question posed in Yes, last sentence of the introduction. answerable form?

4 Is the economic study design appropriate to the Yes, an appropriate study design is used. The stated objective? economic study design is a full economic evaluation (comparison of costs and effects of 2 interventions).

5 Are the structural assumptions and the validation NA (trial-based EE) methods of the model properly reported?

6 Is the chosen time horizon appropriate in order to No, a three-months' time horizon is too short; for a include relevant costs and consequences? societal perspective a time frame of at least one year is generally accepted.

7 Is the actual perspective chosen appropriate? Yes, the societal perspective is preferred in the Netherlands.

8 Are all important and relevant costs for each Yes, (see method section: protocol -driven alternative identified? Intervention costs, healthcare costs, patient and family costs and productivity costs are included.
}

9 Are all costs measured appropriately in physical Yes, cost diaries were used. units?

10 Are costs valued appropriately?

Yes, sources of valuation including the reference year mentioned.

11 Are all important and relevant outcomes for each Yes, outcomes are relevant and they fit the research alternative identified?

12 Are all outcomes measured appropriately? question and perspective.

13 Are outcomes valued appropriately? Yes, measurement instruments are relevant and described.

Yes (see method section) EQ-5D using a Dutch utilitytariff was used.

14 Is an appropriate incremental analysis of costs and Yes, ICER/ICUR are calculated. outcomes of alternatives performed?

15 Are all future costs and outcomes discounted NA when using a time frame for analyses of less than appropriately? one year, discounting is not needed.

16 Are all important variables, whose values are Sub, notall parameters. uncertain, appropriately subjected to sensitivity analysis?

17 Do the conclusions follow from the data reported? Yes, conclusions are supported by the data.

18 Does the study discuss the generalizability of the Yes, mentioned and (briefly) discussed in the results to other settings and patient/client groups? discussion section of the manuscript.

19 Does the article/report indicate that there is no Yes, authors report having no conflict of interest. potential conflict of interest of study researcher(s) and funder(s)?

20 Are ethical and distributional issues discussed Yes, the study was approved by the Medical Ethics appropriately? Committee of Maastricht University. 
Furthermore, the GRADE recommends that the confidence in effect estimates for each important or critical economic outcome should be appraised explicitly, using the same criteria as for health outcomes, so evidence derived from randomized trials starts at high quality, and evidence derived from observational studies starts at low quality. ${ }^{28}$ In addition to integrating economic evidence in CPG development, using the previously described risk of bias checklist is complementary to the GRADE-approach in assisting CPG developers in their deliberations. ${ }^{29}$ Accordingly, to overcome the lack of compatibility with model-based EEs in the GRADE-approach, the NICE has developed a checklist, specifically developed for the UK, composed of items from the CHEC and the Philips checklist. This composite checklist consists of 10 items regarding applicability and 12 items on study limitations (see Appendix $\mathrm{H}$ of the NICE Guidelines Manual. ${ }^{27}$ One should be aware, however, that this list is based solely on expert opinion.

Overall, it can be concluded that when using the GRADE approach in developing CPGs, there is a way to systematically incorporate economic evidence from a partial EE. For further reading on this topic we recommend checking the GRADE website (http://www.gradeworkinggroup.org/). In addition to incorporating economic evidence into CPG development, it is important to perform a complementary assessment on study applicability and the possible limitations for CPG using the NICE checklist. ${ }^{27}$ As the NICE checklist is specifically designed for the UK, some minor adjustments are necessary to use it across jurisdictions, paying attention to such factors as the preferred perspective, the discount rate, or the preferred source of preference data.

\section{Step 3.3 of the overall framework: Transferability assessment of economic evaluations (for both multi-purpose SR-EEs and SR-EEs for guideline development)}

When conducting a SR to identify EEs which are applicable to a specific country or setting, or when developing a CPG and one is interested in cost-effectiveness or cost-utility data, it is important to determine the transferability and generalizability of such studies. Transferability is referred to as the extent to which the results of a study hold true for a different population or setting. ${ }^{30}$ For example, results derived from a study conducted in a developed country will not be representative for use in a developing country. Generalizability is defined as the extent to which the results of a study can be generalized to the population from which the sample was drawn. ${ }^{30}$ Although theoretically there is a clear difference between both concepts they are often used interchangeably. To determine the transferability of a study it's important to know what country-specific pharmacoeconomic guidelines exist and what the differences are between countries. To obtain information regarding country-specific pharmacoeconomic guidelines, the International Society of Pharmacoeconomics and Outcomes Research (ISPOR) has developed a comparative table of 33 countries, including key features for several (mostly 
European \& American) countries (http://www.ispor.org/peguidelines/index.asp). For example, one should pay attention to the tariff used to derive QALYs, which is one of the key transferability issues within cost-utility analyses, or to the perspective used and to the referred discount rates.

However, solely having the key features available for a country (or for a local setting) is not enough for most researchers to assess the transferability of an EE. Accordingly, as is the case for the risk of bias assessment, several instruments exist to evaluate the transferability of an EE (see Online supplementary material II).

In a SR, Goeree et al., ${ }^{31}$ identified 7 checklists for determining the transferability of EEs. Based on the same criteria as the risk of bias checklists, an assessment of these checklists and the full overview can be found in Online supplementary material II. In comparison to the risk of bias checklists, these checklists focus mainly on decision-making and on the implementation of study results in a particular setting. The checklist of Welte et al. was found to be a convenient list because it has clear cutoff points and can be used for the assessment of both trial- and model-based EEs. It has been applied successfully in the past $^{32}$ and the model has been tested extensively by Knies et al.. ${ }^{30}$ The Welte checklist ${ }^{33}$ consists of 3 general knock-out criteria which need to be considered before proceeding to 14 specific knock-out criteria (see Table 6.5). The Drummond (2009) checklist is largely based on the Welte checklist, and the two checklists differ only slightly in their application and content. Accordingly, overall, using the Welte checklist can be recommended. In addition, one should be aware that it is important to discuss the transferability of a particular study with clinicians as clinical practice might vary between countries.

In Table 6.5 an example is provided of how one may conduct the appraisal of a study using the Welte checklist. In this example, results of the study of De Kinderen et al. ${ }^{18}$ are hypothetically transferred from the Netherlands to the UK setting. As can be seen from the example, the difference in perspective between the UK and the Netherlands may lead to the cost-effectiveness ratio being either too high or too low. In this case, one should recalculate the cost-effectiveness ratio excluding costs outside the healthcare setting.

\section{Usability of the different checklists for both multi-purpose SR-EEs and SR-EEs for guideline development}

We provide general recommendations regarding which checklist to use for assessing risk of bias and transferability. These recommendations are based on a balance of the various aspects as described in the previous section. However, to determine what checklist fits best, several other study-specific characteristics determine the actual decision of which list or lists to select. These are, for instance, the time available for the review, the experience of the reviewers, the audience of the $S R$, and the purpose the checklist is designed for in relation to the aim of the review. In addition, the number of items and the time needed to complete a checklist are important factors in determining the applicability of a checklist. For example, the checklist of Philips ${ }^{21}$ is often referred to by CRD as an instrument for appraising the risk of bias within modelling studies, ${ }^{15}$ although it is often ignored, due to 
the large number of criteria (61 items). Accordingly, it is important to look at feasibility when choosing the most appropriate checklist(s).

Moreover, one should be aware that raters are a relevant source of variability ${ }^{34}$; this highlights the importance of multiple raters (at least two) so that discrepancies can be resolved through consensus meetings between raters. In practice, this implies that, in addition to having multiple raters, a few studies (i.e. two or three) should be used to pilot the assessment between multiple raters, after which discrepancies should be discussed between raters to ensure a more uniform assessment strategy (see also Step 2.4 Thielen et al. $^{35}$ and van Mastrigt et al. ${ }^{1}$ ).

Table 6.5 Example using the Welte checklist to determine the transferability of the study of the economic evaluation of the ketogenic diet from the Netherlands to the UK setting (based on de Kinderen et al. 2016)

\begin{tabular}{|c|c|c|}
\hline \multirow[b]{2}{*}{ General knock-out criteria } & \multicolumn{2}{|c|}{$\begin{array}{l}\text { Correspondence between study (the Netherlands) and } \\
\text { decision country (UK) }\end{array}$} \\
\hline & & \\
\hline $\begin{array}{l}\text { The evaluated technology is not } \\
\text { comparable to the one that shall be used in } \\
\text { the decision country. }\end{array}$ & NA & Passed \\
\hline $\begin{array}{l}\text { The comparator is not comparable to the } \\
\text { one that is relevant to the decision country. }\end{array}$ & NA & Passed \\
\hline \multirow{2}{*}{$\begin{array}{l}\text { The study does not possess an acceptable } \\
\text { quality. }\end{array}$} & NA & Passed \\
\hline & $\begin{array}{l}\text { Correspondence between } \\
\text { study (the Netherlands) and } \\
\text { decision country (UK) }\end{array}$ & $\begin{array}{l}\text { ICER of decision country } \\
\text { based on ICER of study } \\
\text { country is: }\end{array}$ \\
\hline \multicolumn{3}{|l|}{ Methodological characteristics } \\
\hline Perspective & Low(healthcare vs societal) & Too low or too high \\
\hline Discount rate & Medium (3.5\% UK vs 4\% NL) & Unbiased (short time horizon) \\
\hline Medical cost approach & High & Unbiased \\
\hline Productivity cost approach & $\begin{array}{l}\text { Low (friction costs method in NL } \\
\text { vs no productivity costs in UK }\end{array}$ & Too low or too high \\
\hline \multicolumn{3}{|l|}{ Healthcare system characteristics } \\
\hline Absolute and relative prices in health care & High & Unbiased \\
\hline Practice variation & Medium & Too low or too high \\
\hline Technology availability & High & Unbiased \\
\hline \multicolumn{3}{|l|}{ Population characteristics } \\
\hline Disease incidence/prevalence & High & Unbiased \\
\hline Case-mix & High & Unbiased \\
\hline Life expectancy & High & Unbiased \\
\hline Health-status preferences & High & Unbiased \\
\hline $\begin{array}{l}\text { Acceptance, compliance, incentives to } \\
\text { patients }\end{array}$ & High & Unbiased \\
\hline Productivity and work-loss time & High & Unbiased \\
\hline Disease spread & High & Unbiased \\
\hline
\end{tabular}

$N A=$ Not applicable; ICER = Incremental cost-effectiveness ratio; NL: the Netherlands; UK: United Kingdom 


\section{Expert commentary}

The starting point for the data extraction, risk of bias and transferability assessment phase is the development of the data extraction sheet. This serves as a basis for collecting data from all the articles included under review. For convenience, one should include the selected risk of bias and transferability checklists in the data extraction sheet.

Next, as shown above, several checklists exist for assessing a variety of factors influencing the validity of study results within a particular setting. Accordingly, depending on the purpose of the review, different recommendations can be made. When one is interested in trial-based EEs, taking into account the thoroughness of the developmental process, the user friendliness, feasibility and purpose of each checklist, the CHECextended ${ }^{24}$ and the BMJ checklist ${ }^{23}$ are most convenient to use. However, these checklists are insufficient when one is also interested in appraising model-based EEs. Therefore, although its length makes it cumbersome to apply to a large number of studies, the Philips checklist $^{21}$ should be considered. However, it should be noted that in current literature there seems to be a lack of consensus regarding the best instrument for assessing the risk of bias of model-based EEs.

As stated above, all currently available checklists focus on full EEs, as full EEs are considered to deliver a high quality of evidence. However, especially in CPG, other factors might be considered in addition to cost-effectiveness data, such as the financial implications of the respective treatment. In this case, the use of a partial EE in a SR might be justified. The GRADE approach came up with a method of incorporating economic evidence when developing CPG, but this method is not suitable for model-based EEs. In addition, the GRADE approach focuses on the estimated use of resources, which is only part of a (full) economic evaluation. Therefore, we would recommend performing a complementary assessment on study applicability and the limitations for CPG using the NICE checklist. ${ }^{27}$

Looking at transferability, several checklists have been identified, of which the Welte checklist $^{33}$ has raised the most attention, likely due to the relative ease of application. In addition, the Welte checklist has been thoroughly examined ${ }^{30}$ and the checklist of Drummond et al. ${ }^{36}$ is based on the work of Welte et al.. ${ }^{33}$ If one is particularly interested in assessing the applicability of HTAs to resource allocation decisions, the Grutters checklist ${ }^{37}$ might be a suitable option (see Online supplementary material II). One should keep in mind that, when incorporating economic evidence in a CPG, a transferability check should always be performed.

A summary of the recommendations made in this article can be found in Table 6.6.

The field of risk of bias assessment is developing quickly, resulting in numerous different checklists with different objectives. This article attempts to highlight the most important checklist currently available, but one should be aware of other checklists in this field. For example, although the product of their research is not defined as a checklist, Evers et al. ${ }^{7}$ provide a list of risks of bias in trial-based EEs. Building on this and several other articles such as the Philips checklist, ${ }^{21}$ Adarkwah et al. ${ }^{38}$ have developed the Bias in Economic Evaluation checklist (ECOBIAS), which is a checklist to determine the risk of bias in EEs. 
However, ECOBIAS is directed more toward model-based EEs. The checklist is aimed at providing a full overview of the biases that could occur in model- and trial-based EEs and includes a total of 22 biases, of which 11 are specific for model-based economic studies. Furthermore, for model developers or users of decision models, Vemer et al. ${ }^{39}$ have developed a checklist, called Assessment of the Validation Status of Health-Economic decision models ( $\mathrm{AdViSHE}$ ), which provides model users with a structured view into the validation status of the model, according to a consensus on what good model validation entails. AdViSHE may provide guidance towards additional validation of a model. However, when preparing a SR, using these checklists as add-ons to other risk of bias or transferability instruments will require a good understanding of EEs and will make the risk of bias assessment a time-consuming exercise.

Table 6.6 Recommendations on data extraction, risk of bias, and transferability for a systematic review of EEs for SR-EEs for multi-purpose and CPG development

\begin{tabular}{|c|c|}
\hline \multicolumn{2}{|c|}{ Step 3: Data Extraction, Risk of Bias, and Transferability } \\
\hline \multicolumn{2}{|c|}{ Step 3.1: Data extraction for SR-EEs for multi-purpose and CPG development } \\
\hline \multirow[t]{2}{*}{$\begin{array}{l}\text { Data extraction- } \\
\text { sheet }\end{array}$} & $\begin{array}{l}\text { Adapt the data extraction for every specific study; include all relevant items from the list in } \\
\text { Table 6.1. }\end{array}$ \\
\hline & Include the selected risk of bias and transferability checklists in the data extraction sheet. \\
\hline \multirow[t]{2}{*}{ Data extraction } & $\begin{array}{l}\text { It is recommended to use a picklist to choose the different answers. } \\
\text { Describe items to increase transparency of scoring. }\end{array}$ \\
\hline & $\begin{array}{l}\text { Disaggregated presentation of the results is highly recommended, as well as incremental } \\
\text { cost-effectiveness ratios, in order to facilitate interpretation of the results. }\end{array}$ \\
\hline \multicolumn{2}{|c|}{ Step 3.2: Risk of Bias assessment } \\
\hline \multirow{3}{*}{$\begin{array}{l}\text { Risk of bias } \\
\text { assessment } \\
\text { for multi-purpose } \\
\text { SR-EEs and CPG } \\
\text { development }\end{array}$} & Use $\mathrm{CHEC}$-extended ${ }^{24}$ or BMJ checklist ${ }^{23}$. \\
\hline & $\begin{array}{l}\text { In cases were one is specifically interested in model-based EE and if the expected number } \\
\text { of included studies is low (e.g. }<10 \text { studies; pragmatic decision) the Philips checklist [18] } \\
\text { could be used. }\end{array}$ \\
\hline & $\begin{array}{l}\text { In cases in which the number of included model-based EEs is high (e.g. }>10 \text { studies; } \\
\text { pragmatic decision), the ISPOR checklist is likely to be more practical for reviewing } \\
\text { purposes. }\end{array}$ \\
\hline \multirow[t]{3}{*}{$\begin{array}{l}\text { Risk of bias } \\
\text { assessment for CPG } \\
\text { development }\end{array}$} & $\begin{array}{l}\text { In general, full EEs should be preferred over partial EEs. However, in CPG development } \\
\text { partial EEs can be used to examine financial implications or the budget impact of a } \\
\text { treatment. }\end{array}$ \\
\hline & $\begin{array}{l}\text { In the absence of a full EE, partial EEs may represent important intermediate stages in our } \\
\text { understanding of the costs and consequences of health services programs and therefore } \\
\text { might be convenient. }\end{array}$ \\
\hline & $\begin{array}{l}\text { Whenever possible, when developing CPGs, the GRADE approach should be used to } \\
\text { systematically incorporate economic evidence of (partial) EE. In addition, it is important to } \\
\text { perform a complementary assessment on study applicability and limitations using the NICE } \\
\text { checklist. }^{27}\end{array}$ \\
\hline \multicolumn{2}{|c|}{ Step 3.3: Transferability for SR-EEs for multi-purpose and CPG development } \\
\hline \multirow[t]{2}{*}{ Transferability } & Use the Welte checklist [42]. \\
\hline & $\begin{array}{l}\text { It is important to discuss the transferability of a particular study with clinicians as clinical } \\
\text { practice might vary between countries. }\end{array}$ \\
\hline \multicolumn{2}{|c|}{ Usability for SR-EEs for multi-purpose and CPG development } \\
\hline \multirow[t]{3}{*}{ Appraisal process } & Raters are a relevant source of variability. \\
\hline & $\begin{array}{l}\text { It is important to have multiple raters (at least two) so that discrepancies can be resolved } \\
\text { through consensus meetings. }\end{array}$ \\
\hline & $\begin{array}{l}\text { It is recommended that a few studies (i.e. two or three) should be used to pilot the } \\
\text { assessment between multiple raters, after which discrepancies should be discussed to ensure } \\
\text { a more uniform assessment strategy. }\end{array}$ \\
\hline
\end{tabular}




\section{Five-year view}

Currently, data extraction in SR-EEs is done in several ways and every author focuses on (slightly) different aspects. However, to improve the comparability of studies, there is a need for a more uniform standard with regard to data extraction sheets.

At this point numerous checklists have been developed and applied within the field of SR and CPG development, specifically focused on EE. However, future research might further improve the risk of bias assessment of EEs. One important topic would be the development and validation of a single tool to assess and grade the risk of bias of both trial- and model-based full EEs in health care. Such a risk of bias assessment tool could make a substantial contribution to the field of health economics, as it would assist end users of cost-effectiveness studies to discriminate among the exploding body of literature, and help producers of such studies to establish a clearer standard, potentially encouraging higher quality and greater rigor. ${ }^{19}$ However, to achieve the necessary level of acceptance and use, the new tool must demonstrate validity and reliability. ${ }^{19}$ Accordingly, it is expected that the GRADE-approach will be adjusted to include model-based EE.

A third important topic would be more uniform and widespread guidance in the use of risk assessment instruments (e.g. which checklist should be used in what situation). An internationally supported protocol for the risk of bias assessment of EEs would support comparative analyses between reviews.

Fourth, there are only a limited number of studies looking at the reliability and validity of the discussed checklists. Future research should provide more insights into this matter.

The last topic would be to stress the need for increased transparency within the field of health economic model development, analysis and reporting. This is particularly important for model-based EEs, where it is often difficult to fully grasp all important aspects of the model when reading only the accompanied article. By (freely) providing models (e.g. as online supplementary material) one could increase the transparency and a more reliable risk of bias assessment could be conducted.

\section{Key issues}

- Currently, several checklists exist for assessing the risk of bias and the transferability of EEs.

- All these checklists focus on full EEs. However, when developing CPG, one might be forced to use a partial EE in resource allocation decisions. For this, the GRADE approach is highly recommended. In addition, it is important to perform a complementary assessment on study applicability and limitations using the NICE checklist. $^{28}$

- There is a lack of consensus regarding the best instrument for assessing the risk of bias within a model-based EE. Of the currently available checklists, the Philips checklist ${ }^{21}$ is recommended when it is deemed feasible. 
- The checklist of Welte et al. ${ }^{33}$ should be used when determining transferability.

- There is a need to standardize the methods for data-extraction sheet development, use and filling in for EEs.

- There is a need for more uniform and widespread guidance in the use of risk of bias checklists (e.g. which checklist should be used in what situation). An internationally supported protocol for the risk of bias assessment of EEs would support comparative analyses between reviews.

Future research should focus on the development and validation of a single tool to assess and grade the risk of bias in both trial-and model-based full EEs in health care.

\section{Financial disclosure/Acknowledgements}

This project was financially supported by the Dutch Society of Medical Specialists, (Stichting Kwaliteitsgelden Medisch Specialisten): projectnumber: 329666498, Utrecht, the Netherlands and the Netherlands Organization for Health Research and Development (ZonMw), grant application number 836011018 . The authors declare that they have no competing interests. The authors have no other relevant affiliations or financial involvement with any organization or entity with a financial interest in or financial conflict with the subject matter or materials discussed in the manuscript apart from those disclosed.

\section{Acknowledgements}

The authors would like to thank the other project members of the project: Laura Burgers (Erasmus University Rotterdam, Rotterdam), Jos Kleijnen (Maastricht University, Maastricht), Frederick Thielen (Erasmus University Rotterdam, Rotterdam), Toon Lamberts (Knowledge Institute of Medical Specialists, Utrecht), Wichor Bramer (Erasmus University Rotterdam, Rotterdam) for their valuable feedback on the draft of this paper. Furthermore, the authors would like to thank Barbara Greenberg for her English editing services and the master and bachelor students from the Maastricht University who were willing to provide us feedback on drafts of the paper. 


\section{References}

*Of considerable interest

1. van Mastrigt GA, Hiligsmann M, Arts JJ, Broos PH, Kleijnen J, Evers SM, Majoie MH. How to prepare a systematic review of economic evaluations for informing evidence-based healthcare decisions: A five-step approach (part 1/3). Expert Rev Pharmacoecon Outcomes Res. 2016; 16(6):689-704.

2. Terrace L. Development and validation of an international appraisal instrument for assessing the quality of clinical practice guidelines: the AGREE project. Qual Saf Health Care 2003;2:18-23.

3. Brouwers MC, et al. AGREE II: advancing guideline development, reporting and evaluation in health care. Canadian Medical Association Journal, 2010;12(18):E839-42.

4. Cncer, C.p.a. AGREE II Domains, Items, Examples and Resources. 19-02-2016]; Available from: http://www.cancerview.ca/cv/portal/Home/TreatmentAndSupport/TSProfessionals/ClinicalGuidelines /GRCMain/GRCAGREEII/GRCAGREEIIDomain3Rigour?_afrLoop=7202305453745000\&jsessionid=2 4h456yAq7d254qiOILI7VsmaXiA_PrK-x3k9VkKOzLOzM5cClFs\%2 1 -

1278458346\&lang=en\&_afrWindowMode=0\&_adf.ctrl-state=y4y9grh4m_4.

5. Drummond MF, et al. Methods for the economic evaluation of health care programmes. 2015: Oxford university press.

* Well-recommened handbook for anyone who is interested in economic evaluations

6. Brown GW. On Small-Sample Estimation. 1947:582-5.

7. Evers SM, Hiligsmann M, Adarkwah CC. Risk of bias in trial-based economic evaluations: Identification of sources and bias-reducing strategies. Psychol Health 2015;30(1):52-71.

8. Husereau $D$, et al. Consolidated health economic evaluation reporting standards (CHEERS) statement. BMC Med 2013;11(1):80.

9. Rennie D, Luft HS. Pharmacoeconomic analyses: making them transparent, making them credible. JAMA, 2000;283(16):2158-60.

10. Higgins JP, et al. The Cochrane Collaboration's tool for assessing risk of bias in randomised trials. BMJ 2011;343:d5928.

11. Begg $C$, et al. Improving the quality of reporting of randomized controlled trials: the CONSORT statement. JAMA 1996;276(8):637-9.

12. Von Elm E, et al. The Strengthening the Reporting of Observational Studies in Epidemiology (STROBE) statement: guidelines for reporting observational studies. Prev Med 2007;45(4):247-51.

13. Schünemann $\mathrm{H}$, et al. The GRADE approach and Bradford Hill's criteria for causation. J Epidemiol Community Health $2011 ; 65(5): 392-5$.

14. Grant MJ, Booth A. A typology of reviews: an analysis of 14 review types and associated methodologies. Health Info Libr J 2009;26(2):91-108.

15. Akers J, Aguiar-lbáñez R, Baba-Akbari Sari A. CRD's Guidance for Undertaking Reviews in Health Care. York (UK): Centre for Reviews and Dissemination (CRD), 2009.

* Well-recommended handbook for conducting a systematic review in general. Developed by the Center for Reviews and Dissemination, York University.

16. Higgins JP, Green S. Cochrane handbook for systematic reviews of interventions. Vol. 5. 2008: Wiley Online Library.

* Well-recommended handbook for conducting a systematic review in general. Developed by the Cochrane Collabarations, an organization specialized in high quality systematic reviews.

17. Mlika-Cabanne $\mathrm{N}$, et al. Sharing hard labour: developing a standard template for data summaries in guideline development. BMJ Qual Saf $2011 ; 20(2): 141-5$

18. de Kinderen RJ, et al. An economic evaluation of the ketogenic diet versus care as usual in children and adolescents with intractable epilepsy: An interim analysis. Epilepsia 2016;57(1):41-50.

19. Walker DG, et al. Best practices for conducting economic evaluations in health care: a systematic review of quality assessment tools. 2012.

* Thorough review on quality assessment tools

20. Sculpher M, Fenwick E, Claxton K. Assessing quality in decision analytic cost-effectiveness models. Pharmacoeconomics 2000;17(5):461-77. 
21. Philips Z, et al. Good practice guidelines for decision-analytic modelling in health technology assessment. Pharmacoeconomics 2006;24(4):355-71.

22. Caro JJ, et al. Questionnaire to assess relevance and credibility of modeling studies for informing health care decision making: an ISPOR-AMCP-NPC Good Practice Task Force report. Value Health 2014 17(2):174-82.

23. Drummond MF, Jefferson T. Guidelines for authors and peer reviewers of economic submissions to the BMJ. BMJ 1996;313(7052):275-83.

24. Evers $S$, et al. Criteria list for assessment of methodological quality of economic evaluations: Consensus on Health Economic Criteria. Int J Technol Assess Health Care 2005;21 (02):240-5.

25. Odnoletkova I, et al. Cost-effectiveness of therapeutic education to prevent the development and progression of type 2 diabetes: Systematic review. J Diabetes Metab 2014. 2014.

26. Consensus Health Economic Criteria - CHEC list Available from: https://hsr.mumc.maastrichtuniversity.nl/consensus-health-economic-criteria-chec-list.

27. Excellence, N.i.f.C. Developing NICE guidelines: the manual. 2014; Available from: http://www.nice.org.uk/article/PMG20/chapter/7-Incorporating-economic-evaluation.

28. Brunetti $M$, et al. GRADE guidelines: 10. Considering resource use and rating the quality of economic evidence. J Clin Epidemiol 2013;66(2):140-50

29. Brunetti $M$, et al. Grading economic evidence. 2010: Wiley-Blackwell: Oxford.

30. Knies $S$, et al. The transferability of economic evaluations: Testing the model of Welte. Value Health, 2009; $12(5): 730-8$

31. Goeree R, et al. Transferability of health technology assessments and economic evaluations: a systematic review of approaches for assessment and application. ClinicoEconomics and outcomes research: CEOR, $2011 ; 3: 89$.

32. Essers BA, et al. Transferability of Model-Based Economic Evaluations: The case of trastuzumab for the adjuvant treatment of HER2-positive early breast cancer in the Netherlands. Value Health 2010;13(4): 375-80.

33. Welte $R$, et al. A decision chart for assessing and improving the transferability of economic evaluation results between countries. Pharmacoeconomics 2004;22(13):857-76.

34. Müller D, et al. Reporting health care decision models: a prospective reliability study of a multidimensional evaluation framework. Expert review of pharmacoeconomics \& outcomes research, 2015:1-9.

35. Thielen FW, Van Mastrigt G, Burgers LT, Bramer WM, Majoie H, Evers S, Kleijnen J. How to prepare a systematic review of economic evaluations for informing evidence-based healthcare decisions: Expert Rev Pharmacoecon Outcomes Res. 2016; 16(6):705-21.

36. Drummond $M$, et al. Transferability of economic evaluations across jurisdictions: ISPOR Good Research Practices Task Force report. Value Health 2009; 12(4):409-18.

37. Grutters JP, et al. Bridging trial and decision: a checklist to frame health technology assessments for resource allocation decisions. Value Health 2011 ; 14(5):777-84.

38. Adarkwah CC, et al. Risk of bias in model-based economic evaluations: the ECOBIAS checklist. Expert review of pharmacoeconomics \& outcomes research, 2015:1-11.

39. Vemer P, et al. AdViSHE: A validation-assessment tool of health-economic models for decision makers and model users. PharmacoEconomics 2015:1-13. 


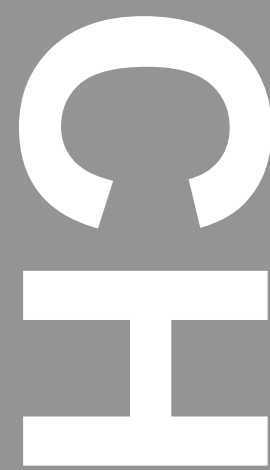

A systematic review of economic evaluations of treatments for patients with

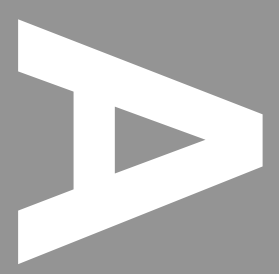
epilepsy
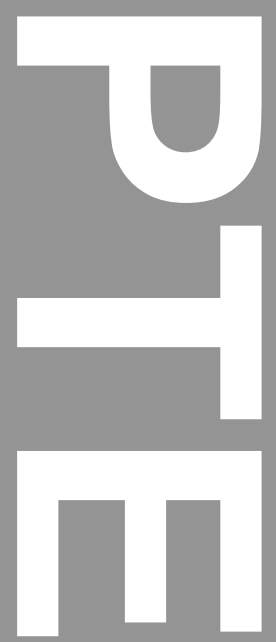

B.F.M. Wijnen G.A.P.G. van Mastrigt

S.M.A.A. Evers O. Gershuni

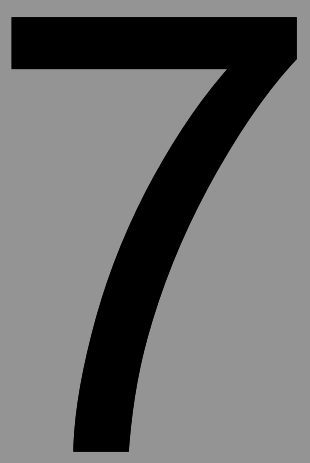

D.A.J.E. Lambrechts H.J.M. Majoie D. Postulart B.A.P. Aldenkamp R.J.A. de Kinderen Epilepsia doi: $10.1111 /$ epi. 13655 


\section{Abstract}

\section{Introduction}

The increasing number of treatment options and the high costs associated with epilepsy have fostered the development of economic evaluations in epilepsy. It is important to examine the availability and quality of these economic evaluations and to identify potential research gaps. As well as looking at both pharmacological (anti-epileptic drugs) and nonpharmacological (e.g. epilepsy surgery, ketogenic diet, vagus nerve stimulation) therapies, this review examines the methodological quality of the full economic evaluations included.

\section{Methods}

Literature search was performed in MEDLINE, EMBASE, NHS Economic Evaluation Database (NHS EED), Econlit, Web of Science and CEA Registry. In addition, Cochrane Reviews, Cochrane DARE and Cochrane Health Technology Assessment Databases were used. To identify relevant studies, predefined clinical search strategies were combined with a search filter designed to identify health economic studies. Specific search strategies were devised for the following topics: 1) anti-epileptic drugs (AED); 2) patients with cognitive deficits; 3) elderly patients; 4) epilepsy surgery; 5) ketogenic diet; 6) vagus nerve stimulation; 7) treatment of (non)convulsive status epilepticus.

\section{Results}

A total of 40 publications were included in this review, $29(73 \%)$ of which were articles about pharmacological interventions. Mean quality score of all articles on the CHEC-extended was $81.8 \%$, the lowest quality score being $21.05 \%$ while 5 studies had a score of $100 \%$. Looking at the Consolidated Health Economic Evaluation Reporting Standards (CHEERS), the average quality score was $77.0 \%$, the lowest being $20.0 \%$ and four studies rated as $100 \%$.

\section{Conclusion}

There was a substantial difference in methodology in all included articles which hampered the attempt to combine information meaningfully. Overall, the methodological quality was acceptable; however, some studies performed significantly worse than others. The heterogeneity between the studies stresses the need to define a reference case (e.g. how should an economic evaluation within epilepsy be performed) and to derive consensus on what constitutes "standard optimal care". 


\section{Introduction}

Epilepsy is a chronic neurological disorder of the brain which involves recurrent seizures as a result of sudden excessive electrical discharges in a group of brain cells. The site of such discharges determines the clinical manifestation. Hence, clinical manifestations of seizures vary, depending on where the disturbance starts and how far it spreads. Epilepsy is the most common serious neurological disorder with a global prevalence of almost 60 million ${ }^{1}$ and an annual incidence of 50.4 per 100,000 persons. $^{2}$

The increasing number of treatment options and the high costs associated with epilepsy have fostered the development of economic evaluations in this field. Economic evaluations compare costs and consequences of health care interventions: for example, by examining the added value (in both costs and effects) of anti-epileptic drugs (AED) or electric stimulation such as the vagus nerve stimulation (VNS) or deep brain stimulation (DBS).

The goal of good health policy-making is to achieve health benefits and therefore to 'produce' health. In order to make good choices in health care, policy-makers try to find a balance between costs and effects of interventions. Economic evaluations provide a solution for this assessment, as they provide information about the relative efficiency of choosing between alternative interventions. In a "full" economic evaluation, the additional benefits of a health care programme are weighed against the additional costs compared to a reference programme. For example, an economic evaluation will reveal whether a new AED is 'good value for money' compared to existing alternatives.

Economic evaluations are important at different levels of decision-making. An increasing number of countries are using cost-effectiveness as a criterion for deciding whether an intervention, especially pharmaceuticals, should be reimbursed. ${ }^{3}$ Hence, in some countries, economic assessments are now of such high importance that a drug will not be refunded if no economic evaluation has been conducted. But also at the level of national healthcare services or at the level of the individual patient, economic evaluations can help in decision-making. The principle of economic evaluation is simple: determine the cost/benefit ratio and select the intervention for which this ratio is the lowest. Conducting a high quality full economic evaluation is, however, no sinecure.

The aim of this review is to present an overview of published full economic evaluations of all healthcare interventions for patients with epilepsy. The treatments assessed in this review will be categorized according to the topics covered in the clinical practice guidelines (CPG) as developed by the International League Against Epilepsy (ILAE) and the National Institute for Care Excellence (NICE) for treatment of epilepsy. ${ }^{4,5}$ This review looks at both pharmacological (i.e. anti-epileptic drugs) and non-pharmacological therapies (e.g. epilepsy surgery, ketogenic diet and vagus nerve stimulation) and devotes a specific section to vulnerable groups (i.e. elderly and children). In addition, it includes both trial-based and model-based full economic evaluations. Furthermore, the methodological quality will be determined of published full economic evaluations of all healthcare interventions for patients with epilepsy. 


\subsection{Essentials of economic evaluations}

The general approach of an economic evaluation is to compare the consequences of health care programmes with their costs. The term "full EE" refers to the comparative analysis of alternative courses of action in terms of both costs (resource use) and consequences (outcomes, effects). ${ }^{6}$ An Incremental Cost-Effectiveness Ratio (ICER) is then calculated for each treatment being assessed, where the denominator reflects the incremental gain in effects, benefits or utilities, and the numerator reflects the additional cost of achieving that health gain.

\subsection{Types of economic evaluation}

There are four types of full economic evaluation, namely: Cost-Minimization Analysis (CMA), Cost-Benefit Analysis (CBA), Cost-Effectiveness Analysis (CEA) and Cost-Utility Analysis (CUA). ${ }^{6}$ In CMA, the effects of the interventions of interest are assumed to be equal; one is, therefore, only interested in a reduction in costs. However, in cases where studies just assume equal effectiveness without examining it, it is questionable whether one can speak of a full EE. In a CBA, attempts are made to place a value on the consequences of programmes in monetary terms, in order to make them commensurate with the costs. Potentially, this is the broadest programme for justifying the costs. Expressing benefits in monetary terms is, however, often hampered by measurement problems. In a CEA, the consequences of programmes are measured in terms of the appropriate natural effects of physical units, such as '50\% seizure reduction' or 'seizure-free patients'. The main argument for these clinical measures is the relative ease of measurement and interpretation. One of the major limitations of the CEA and its disease-specific outcome is that it does not permit comparisons with interventions evaluated in other disease areas (e.g. comparing blood pressure in hypertension treatment and seizure frequency in epilepsy treatment). In a CUA, the consequences of programmes are adjusted by health state preference scores or utility weights; that is, states of health associated with the outcomes are valued relative to one another. The most common measure of consequences in a CUA is the Quality-Adjusted Life-Year (QALY). ${ }^{6}$ A QALY incorporates effects in terms of both quality of life (utilities) and survival (life-years gained). In contrast to the outcome measure of the CEA, it is a generic outcome measure. To calculate QALYs, it is necessary to represent health on a scale where death and full health are assigned values of 0 and 1 , respectively. As there are many ways of obtaining utilities to calculate QALYs, it is important to look at the methods used or the questionnaire chosen. A disease-specific quality of life measure, for example, is specific to an individual disease or condition and includes dimensions relevant to that disease (e.g. epilepsy-specific QOL measure: QOLIE-89). A generic quality of life measure is a comprehensive measure of health that has a broad range of components and is applicable across different patient groups (e.g. EuroQoL). ${ }^{7}$ Alternatively, other generic outcome measures are sometimes used such as disability adjusted life years (DALYs). DALYs represent the number of years lost due to ill-health, disability or early death. 


\subsection{Study design}

There are two study designs for conducting an economic evaluation, namely trial-based or model-based design. In trial-based economic evaluations, data on resource use and effectiveness are collected simultaneously, for example, alongside a randomized trial. The benefits of this approach are that results reflect actual data from real patients, and the internal validity of the data can be assessed through controlled clinical conditions. A disadvantage is that it may not be possible to generalize the treatment pathways and patient samples to the larger population. To overcome this disadvantage, it is possible to model the costs and outcomes of different treatments. Model-based economic evaluations provide a means of bringing all clinical, costs and quality of life data together in one model by using techniques of epidemiology or decision analysis. The results can, therefore, be generalized in terms of the larger population. Lifetime costs and outcomes can then be estimated.

\subsection{Perspective of analysis}

The perspective indicates from which point of view an economic evaluation is being considered and determines which costs will be examined, and thus which conclusions can be drawn from any particular economic evaluation. A programme that looks unattractive from one viewpoint may look significantly better when other viewpoints are considered. Analytical viewpoints may include any or all of the following: the individual patient, the specific institution, the target group for specific services, the Ministry of Health budget, and the community or societal viewpoint. According to the majority of national guidelines, an economic evaluation study has to be performed from the societal perspective. ${ }^{6,8}$ In this case, all costs, including health care costs, costs of patient and family and costs in other sectors should be measured, irrespective of who benefits from them or who pays for them.

\section{Methods}

A protocol of this systematic review was published in the International Prospective Register of Systematic Reviews (http://www.crd.york.ac.uk/prospero/; registration number CRD42015019278). The PRISMA statement was followed in the reporting of this article. ${ }^{9}$

\subsection{Literature search}

A literature search was performed in MEDLINE (via PubMed), EMBASE, NHS Economic Evaluation Database (NHS EED), Econlit, Web of Science and CEA Registry. In addition, Cochrane Reviews, Cochrane DARE and Cochrane Health Technology Assessment Databases were used. To identify relevant studies, we combined predefined clinical search strategies (to assess effectiveness of treatments) with a search filter constructed by the health information research unit of McMaster University (see: 
http://hiru.mcmaster.ca/hiru/HIRU_Hedges_EMBASE_Strategies.aspx), which is designed to identify health economic studies. In addition, citation tracking was performed for all included studies.

As this review focuses on topics discussed in the CPGs, as developed by the ILAE and NICE for treatment of epilepsy, specific search strategies were devised for the following topics: 1) anti-epileptic drugs; 2) patients with cognitive deficits; 3) elderly patients; 4) epilepsy surgery; 5) ketogenic diet; 6) vagus nerve stimulation; 7) treatment of (non)convulsive status epilepticus in children; and 8) treatment of (non)convulsive status epilepticus in adults.

To examine each subject/sub-area, some "key" references were identified beforehand which had to be identified through the various search strategies in the various databases. The search strategy can be found in Appendix 7.1.

\subsection{Inclusion/exclusion criteria}

The review focused on full economic evaluations comparing different treatment options for patients with epilepsy. In addition, only articles published in English during the period January 2000 - March 2016 were considered. Excluded were those papers focusing on diagnostics.

\subsection{Data extraction \& quality assessment}

Two researchers selected the abstracts and extracted the data independently of each other. Data was extracted using a predefined form which included the following information: study type, year of publication, year of currency and currency type, study setting (country), target population (gender and age), analysis perspective, intervention type, health outcome measure and type of economic evaluation

To determine the quality of the studies, the Consensus on health economics checklist extended (CHEC-extended) was used. This is an extension of the original CHEC checklist to include questions about model-based economic evaluations. ${ }^{10,11}$ To determine the quality of reporting of the included studies, the Consolidated Health Economic Evaluation Reporting Standards (CHEERS) statement was used..$^{12}$ In order to limit the possibility of biased results, two reviewers independently reviewed both the data extraction form and the quality appraisal of the included studies (BFMW and OG). As a consequence, all the articles were double-scored on the data extraction and quality assessment part. Possible differences in scoring were discussed until consensus was reached. Both checklists can be found in Appendix 7.2.

To calculate an overall quality-score for each article based on the $\mathrm{CHEC}$ checklist, each time a "Yes" was scored, 1 point was allocated and each time "suboptimal" was scored, 0.5 points were allocated. The total score per article was then divided by all the applicable items for that particular study. ${ }^{11}$ The same calculation was applied for the CHEERS, the only exception being, there is no "suboptimal" in the CHEERS. 
All costs were converted to Euros $(€)$ and apply to the year 2015 based on estimates from the International Monetary Fund (IMF) World Economic Outlook Database 'GDP deflator index' dataset. This dataset contains 'GDPD values' for 184 countries (currencies) from 1980 onwards. $^{13}$

\section{Results}

\subsection{Search results}

A total of 1474 publications were identified using the different search strategies and after removal of all duplicate studies, 1028 publications were eligible for title and abstract screening. After screening the titles and abstracts for inclusion criteria, 959 articles were excluded. Next, 71 full-text articles were assessed for eligibility, 31 of which were excluded for various reasons: 1) no original research/not a full report $(n=15) ; 2)$ no full economic evaluation $(n=9) ; 3)$ no epilepsy $(n=4)$; 4 ) only focused on diagnostics $(n=3)$. A total of 40 publications were finally included in this review. The flow chart of the literature search is presented in Figure 7.1.

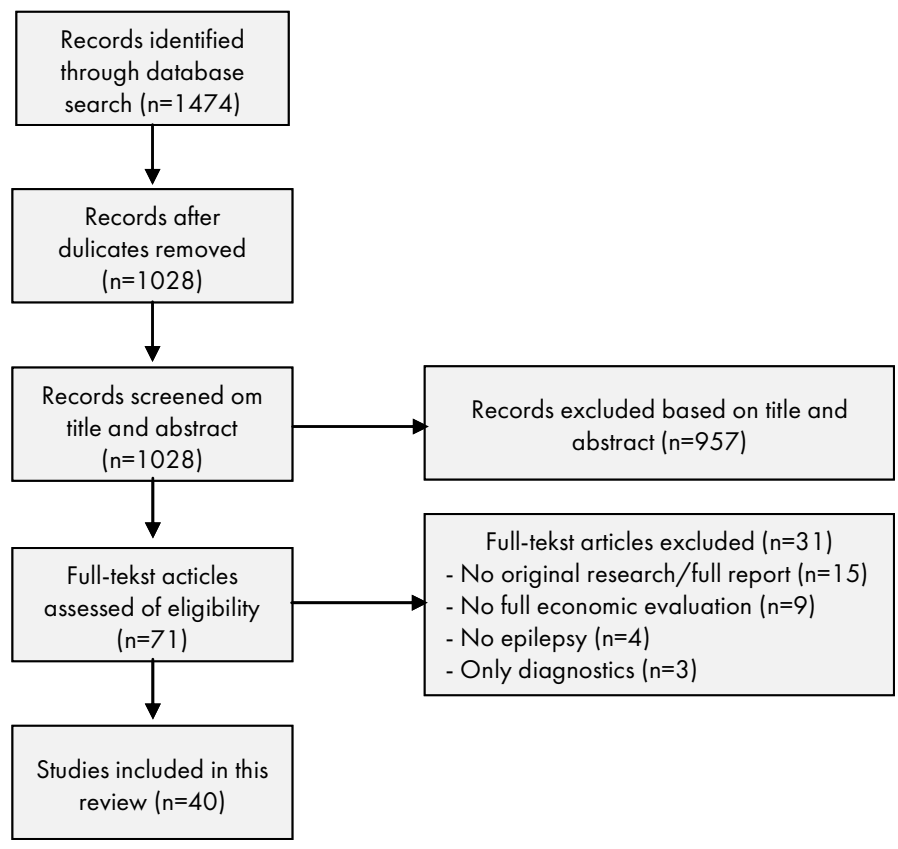

Figure 7.1 Flowchart of study selection. 


\subsection{General characteristics}

The most recent study was from 2016, and the oldest was published in 2000. Most studies were conducted in the United Kingdom ( $n=12 ; 30 \%$ ) followed by the Netherlands $(n=6$; $15 \%)$. The included studies reported cost-effectiveness for the following interventions: different types of AED (29 studies; 73\%), ketogenic diet (2 studies; $5 \%$ ), surgery in patients with refractory epilepsy (3 studies ( $8 \%$ ), self-management ( 3 studies; $8 \%$ ), VNS (2 studies; $5 \%$ ) and lastly drug monitoring in patients with epilepsy ( 1 study; $3 \%$ ).

The most commonly used perspective was the healthcare perspective $(67 \%)$, followed by the societal perspective (10\%). In $28(70 \%)$ of the 40 studies, a model was used to estimate costs and effects. A comprehensive list of all included studies can be found in Table 7.1.

\subsection{Quality of the identified studies}

Mean quality score of all articles on the CHEC-extended was $81.8 \%$, the lowest being $21.05 \%$ while 5 studies had a score of $100 \%$ (see Table 7.2). Furthermore, pharmacological studies scored $81.6 \%$ on average and non-pharmacological studies scored $82.4 \%$ on average. Looking at the CHEERS, the average quality score was $77.0 \%$, the lowest being $22.7 \%$ and four studies rated as $100 \%$ (see Table 7.3). Furthermore, pharmacological studies scored $77.4 \%$ on average and non-pharmacological studies scored $75.5 \%$ on average. Hence, pharmacological studies scored slightly better on the reporting of their study.

\subsection{Pharmacological interventions}

Twenty-nine articles (73\%) on pharmacological interventions or anti-epileptic drugs (AED) were included in the study. Main outcomes and ICERs/ICURs of all studies can be found in Table 7.4.

\subsection{AED as monotherapy}

Knoester et al. ${ }^{14}$ compared six different strategies encompassing carbamazepine, valproic acid and lamotrigine; 1-2) carbamazepine as first-line monotherapy followed by either valproic acid or lamotrigine in the case of carbamazepine failing because of either a lack of seizure control or adverse effects; $3-4$ ) valproic acid as first-line monotherapy followed by either carbamazepine or lamotrigine if valproic acid fails because of either a lack of seizure control or adverse effects; and 5-6) lamotrigine as first-line monotherapy followed by either carbamazepine or valproic acid if lamotrigine fails because of either a lack of seizure control or adverse effects. Using carbamazepine-valproic acid as a reference, the study concludes that this dominates valproic acid-carbamazepine, valproic acidlamotrigine and lamotrigine-carbamazepine; the ICER of carbamazepine-lamotrigine is $€ 7,308$ per complete success, and the ICER of lamotrigine-valproic acid, €48,593 per complete success. 


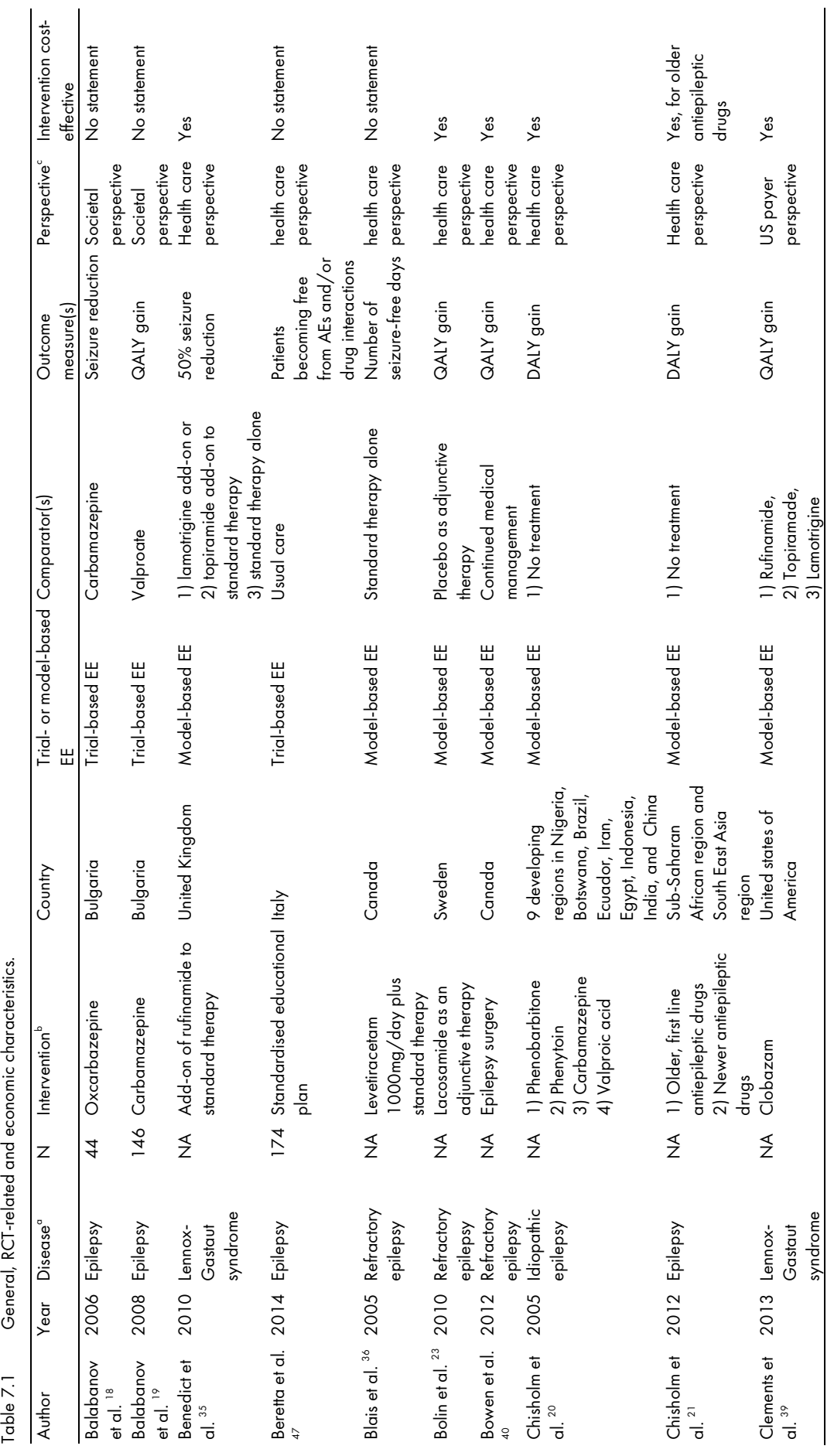




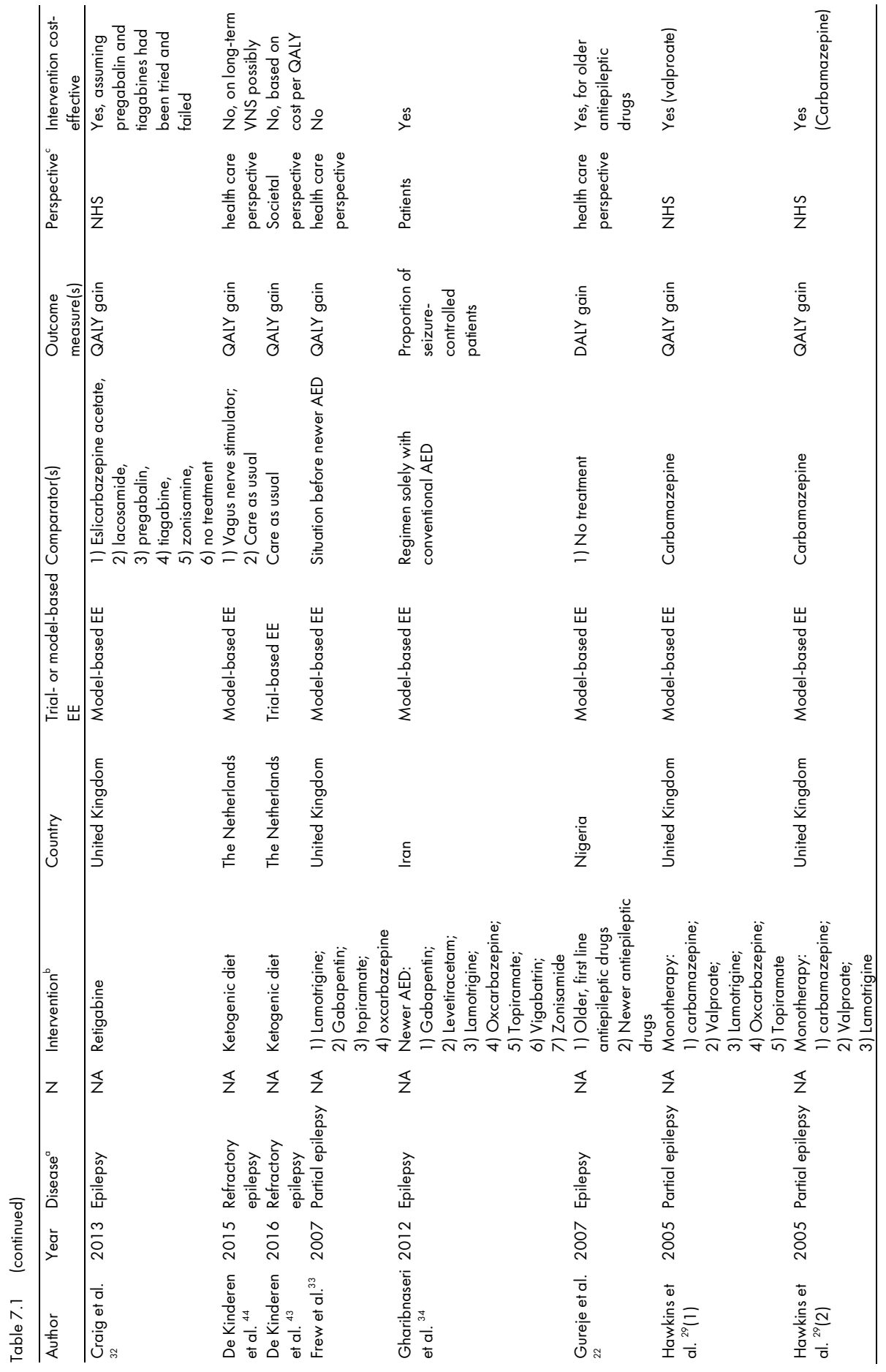




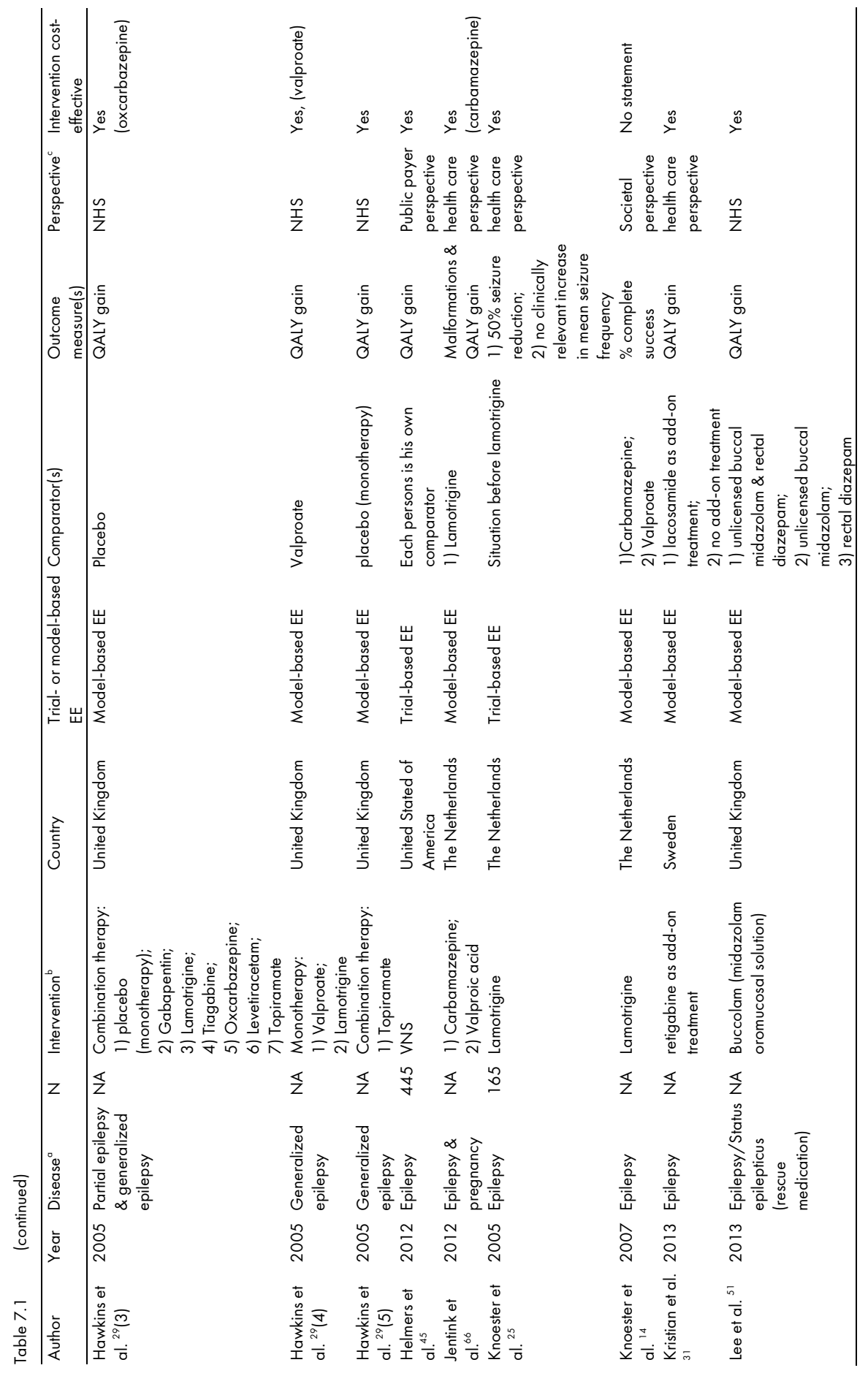




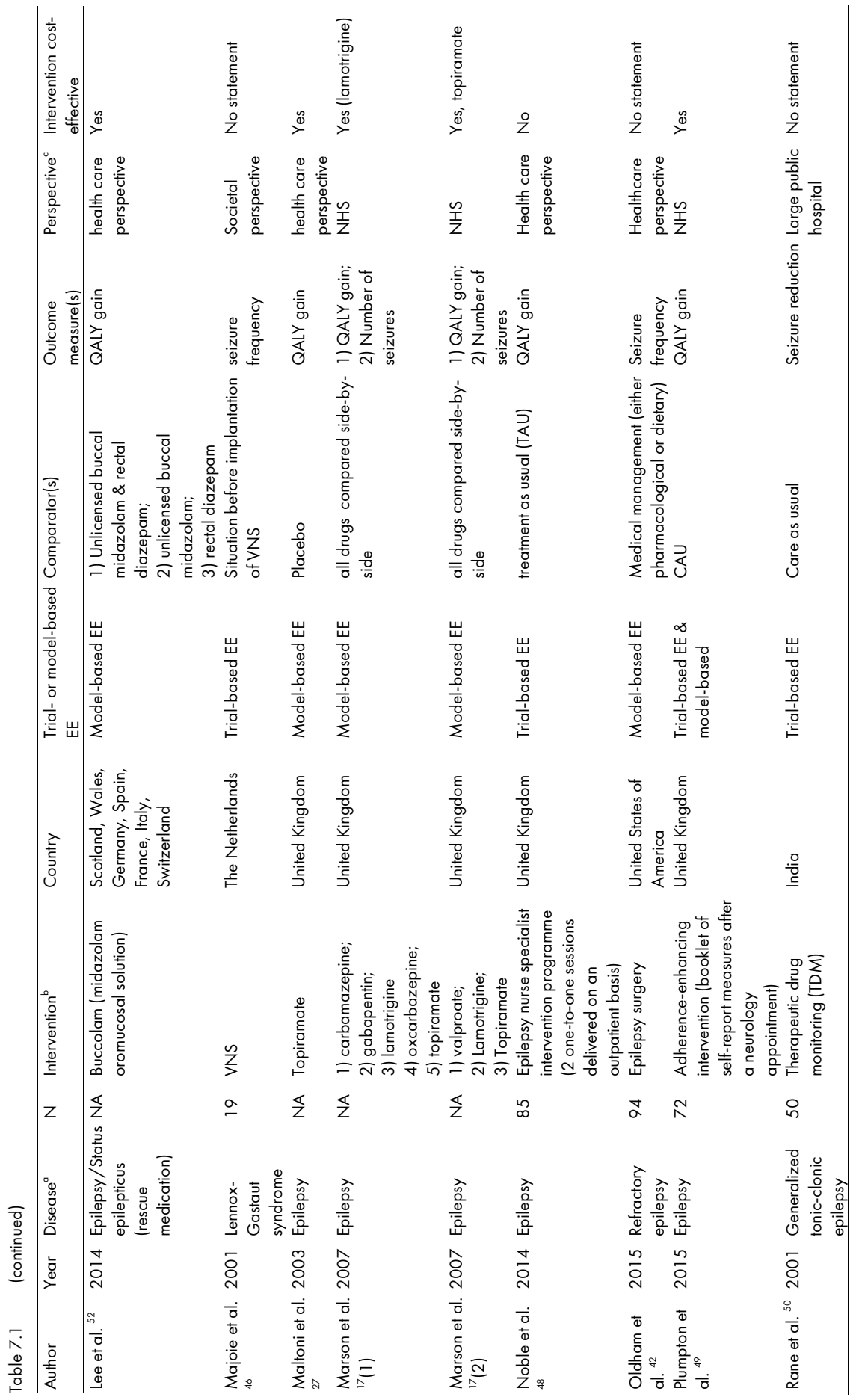




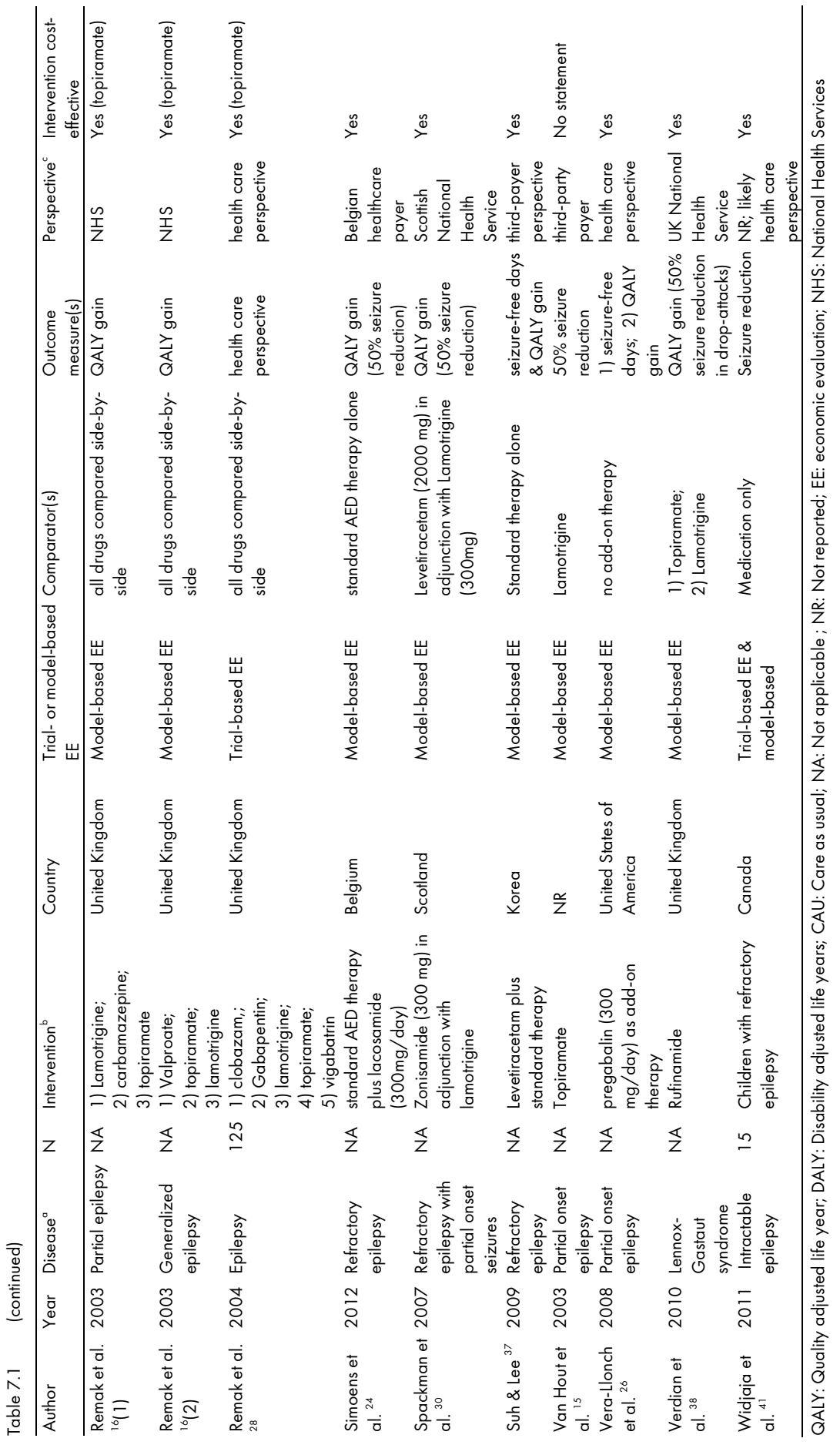




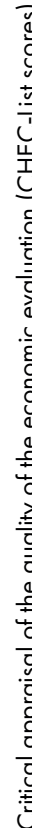

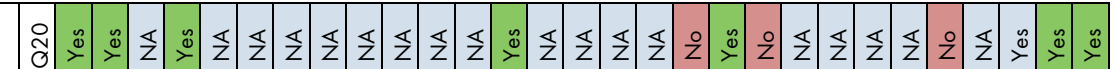

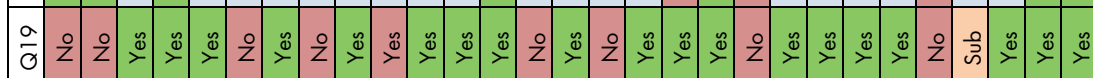

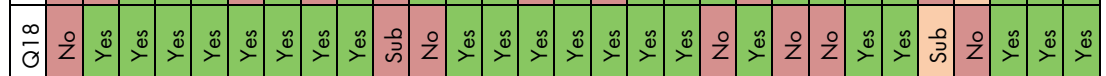

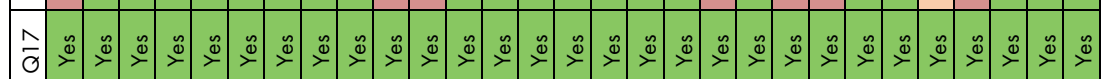

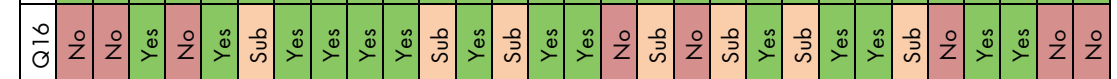

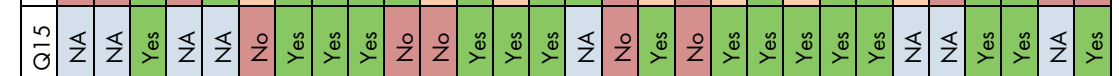

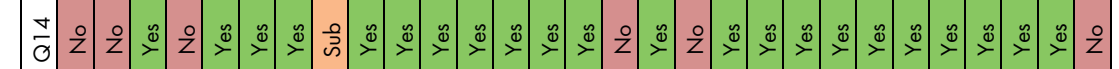

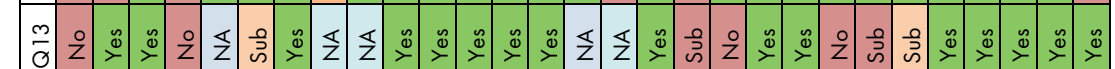

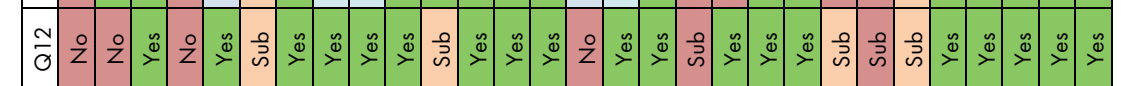

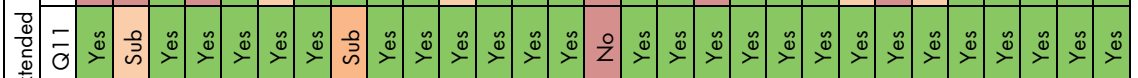

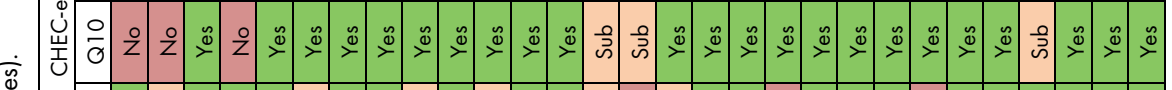

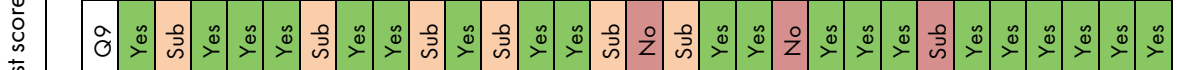
岂

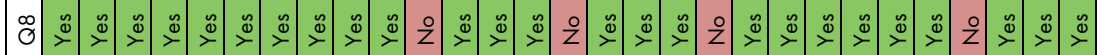
Oे

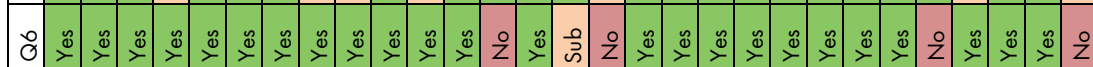

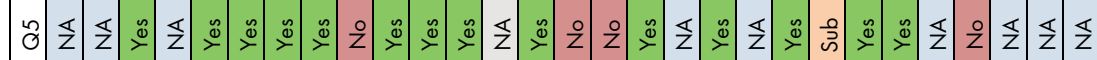

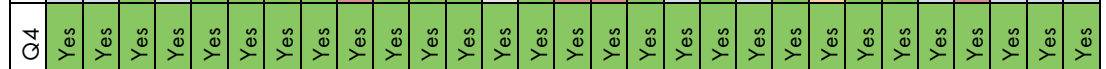
䆓

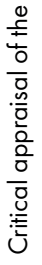

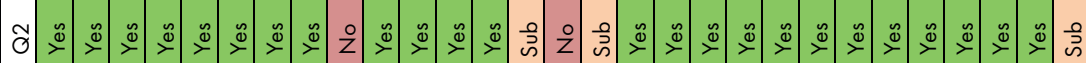

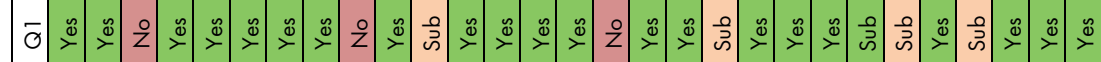

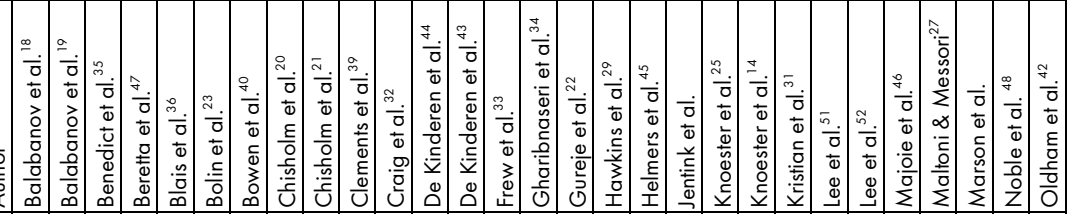




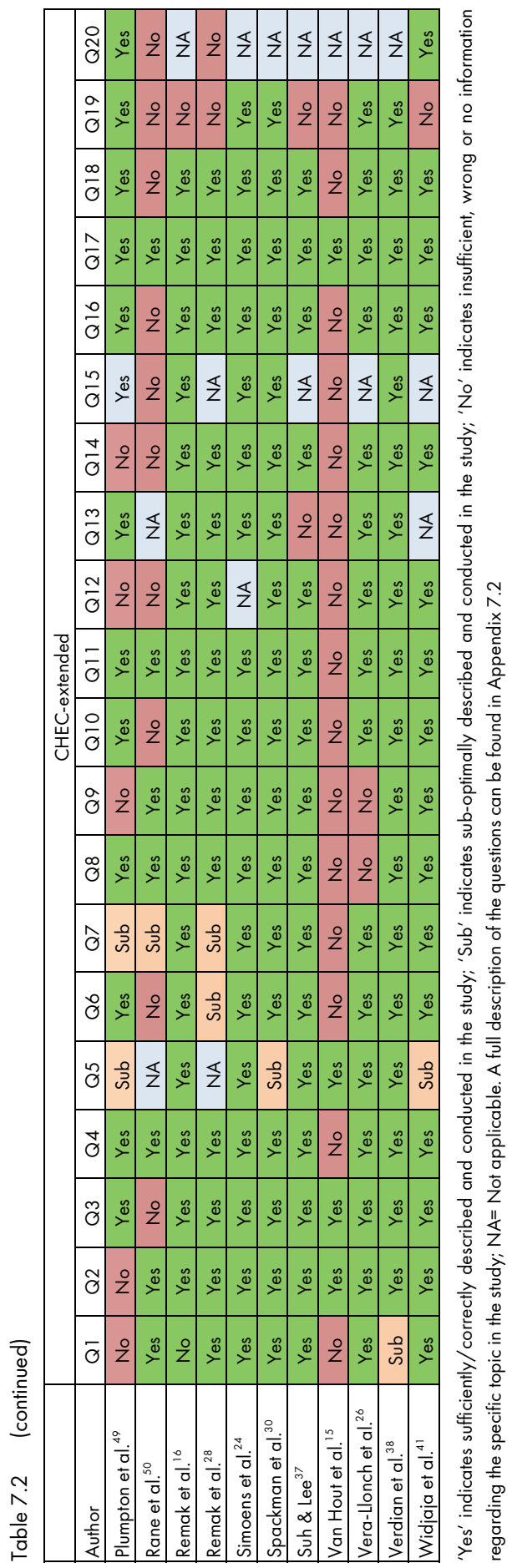


Table 7.3 Consolidated Health Economic Evaluation Reporting Standards (CHEERS)-scores per article.

\begin{tabular}{|c|c|c|c|c|}
\hline \#Yes & \#No & \#NA & Total scores & Author \\
\hline 12 & 8 & 7 & $60.0 \%$ & Balabanov et al. $^{18}$ \\
\hline 12 & 8 & 7 & $60.0 \%$ & Balabanov et al. ${ }^{19}$ \\
\hline 20 & 2 & 5 & $90.9 \%$ & Benedict et al. ${ }^{35}$ \\
\hline 15 & 5 & 7 & $75.0 \%$ & Beretta et al. ${ }^{47}$ \\
\hline 19 & 2 & 6 & $90.5 \%$ & Blais et al. ${ }^{36}$ \\
\hline 17 & 5 & 4 & $77.3 \%$ & Bolin et al. ${ }^{23}$ \\
\hline 22 & 1 & 4 & $95.7 \%$ & Bowen et al. ${ }^{40}$ \\
\hline 16 & 7 & 4 & $69.6 \%$ & Chisholm et al. ${ }^{20}$ \\
\hline 14 & 9 & 4 & $60.9 \%$ & Chisholm et al. ${ }^{21}$ \\
\hline 19 & 4 & 4 & $82.6 \%$ & Clements et al. ${ }^{39}$ \\
\hline 12 & 11 & 4 & $52.2 \%$ & Craig et al. ${ }^{32}$ \\
\hline 21 & 2 & 3 & $91.3 \%$ & De Kinderen et al. ${ }^{44}$ \\
\hline 19 & 1 & 7 & $95.0 \%$ & De Kinderen et al. ${ }^{43}$ \\
\hline 16 & 6 & 5 & $72.7 \%$ & Frew et al. ${ }^{33}$ \\
\hline 16 & 5 & 6 & $76.2 \%$ & Gharibnaseri et al. ${ }^{34}$ \\
\hline 5 & 17 & 5 & $22.7 \%$ & Gureje et al. ${ }^{22}$ \\
\hline 22 & 0 & 4 & $100.0 \%$ & Hawkins et al. ${ }^{29}$ \\
\hline 16 & 5 & 6 & $76.2 \%$ & Helmers et al. ${ }^{45}$ \\
\hline 15 & 7 & 5 & $68.2 \%$ & Jentink et al. \\
\hline 15 & 4 & 8 & $78.9 \%$ & Knoester et al. ${ }^{25}$ \\
\hline 21 & 0 & 6 & $100.0 \%$ & Knoester et al. ${ }^{14}$ \\
\hline 18 & 5 & 4 & $78.3 \%$ & Kristian et al. ${ }^{31}$ \\
\hline 22 & 1 & 4 & $95.7 \%$ & Lee et al. ${ }^{51}$ \\
\hline 19 & 3 & 5 & $86.4 \%$ & Lee et al. ${ }^{52}$ \\
\hline 11 & 8 & 8 & $57.9 \%$ & Majoie et al. ${ }^{46}$ \\
\hline 18 & 5 & 4 & $78.3 \%$ & Maltoni \& Messori ${ }^{27}$ \\
\hline 21 & 1 & 5 & $95.5 \%$ & Marson et al. \\
\hline 19 & 1 & 7 & $95.0 \%$ & Noble et al. ${ }^{48}$ \\
\hline 9 & 11 & 7 & $45.0 \%$ & Oldham et al. ${ }^{42}$ \\
\hline 16 & 6 & 5 & $72.7 \%$ & Plumpton et al. ${ }^{49}$ \\
\hline 4 & 16 & 7 & $20.0 \%$ & Rane et al. ${ }^{50}$ \\
\hline 18 & 5 & 4 & $78.3 \%$ & Remak et al. ${ }^{16}$ \\
\hline 16 & 5 & 6 & $76.2 \%$ & Remak et al. ${ }^{28}$ \\
\hline 23 & 0 & 4 & $100.0 \%$ & Simoens et al. ${ }^{24}$ \\
\hline 20 & 3 & 4 & $87.0 \%$ & Spackman et al. ${ }^{30}$ \\
\hline 18 & 4 & 5 & $81.8 \%$ & Suh \& Lee ${ }^{37}$ \\
\hline 11 & 11 & 5 & $50.0 \%$ & Van Hout et al. ${ }^{15}$ \\
\hline 18 & 5 & 4 & $78.3 \%$ & Vera-Llonch et al. ${ }^{26}$ \\
\hline 23 & 0 & 4 & $100.0 \%$ & Verdian et al. ${ }^{38}$ \\
\hline 18 & 4 & 5 & $81.8 \%$ & Widjaja et al. ${ }^{41}$ \\
\hline $\begin{array}{l}\text { Average quality } \\
\text { score: }\end{array}$ & & & $77.0 \%$ & \\
\hline
\end{tabular}

'Yes' indicates sufficiently/correctly described and conducted in the study; 'No' indicates insufficient, wrong or no information regarding the specific topic in the study; $N A=$ Not applicable. A full description of the items can be found in Appendix 7.2 


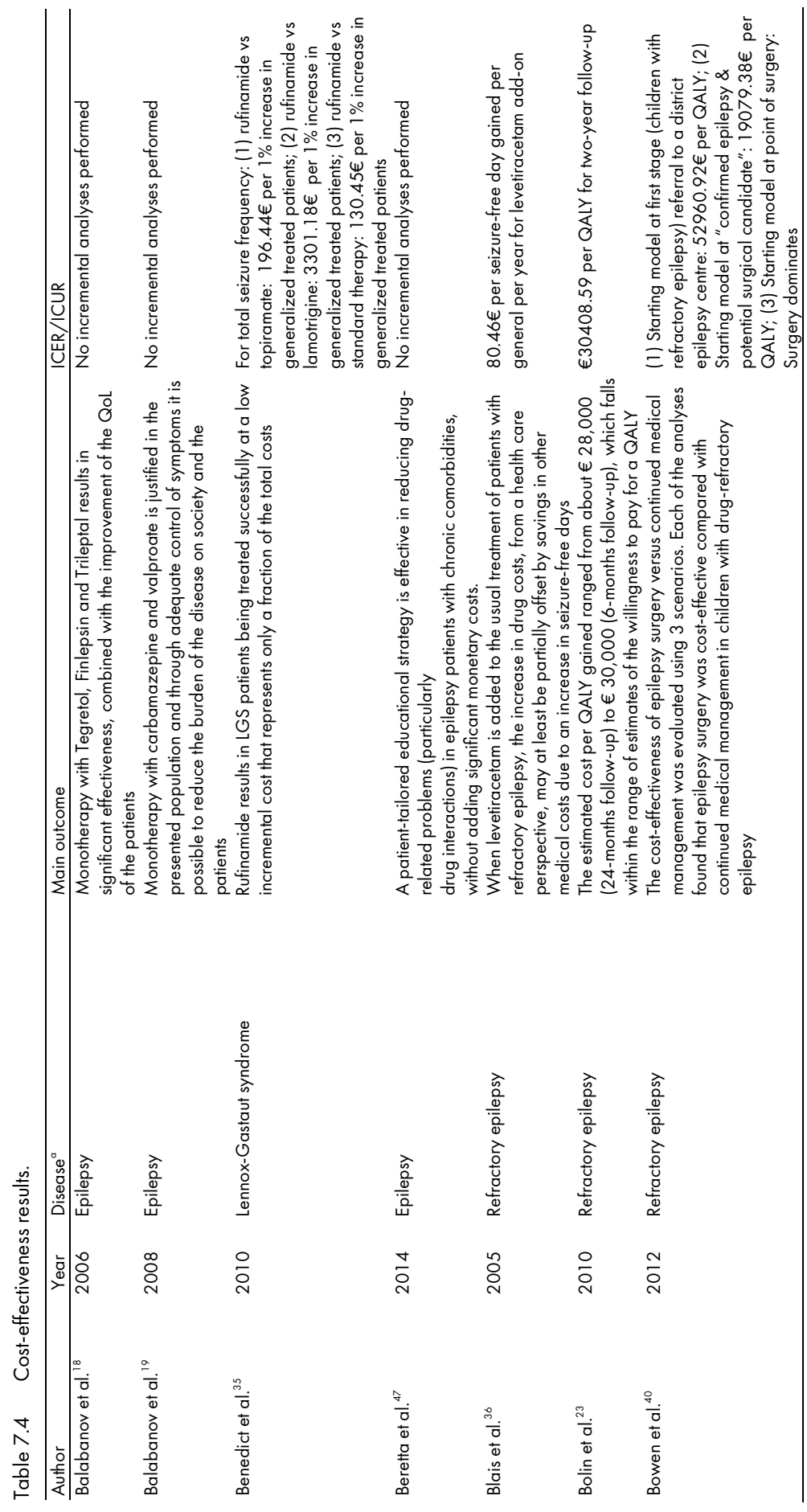




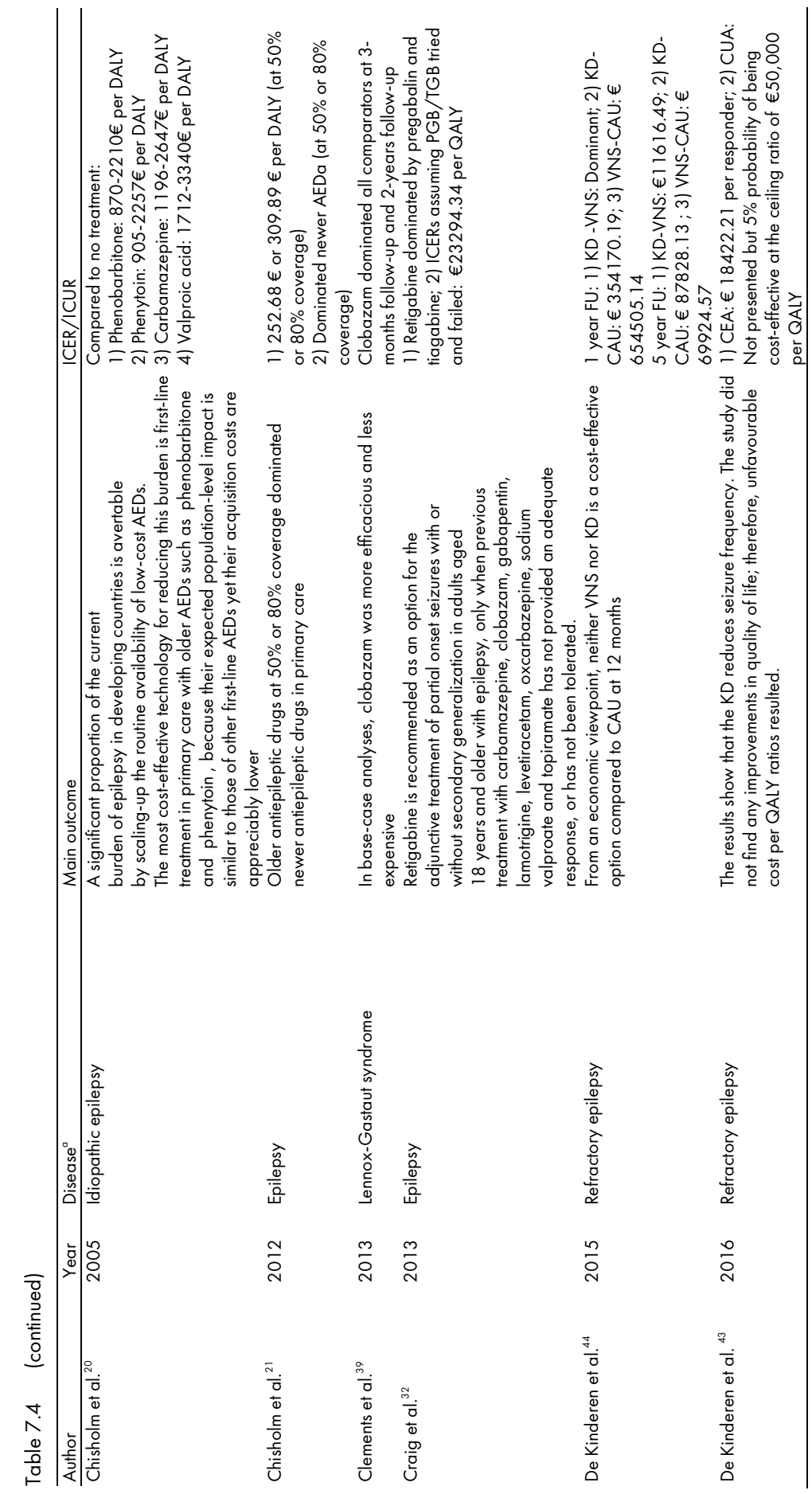




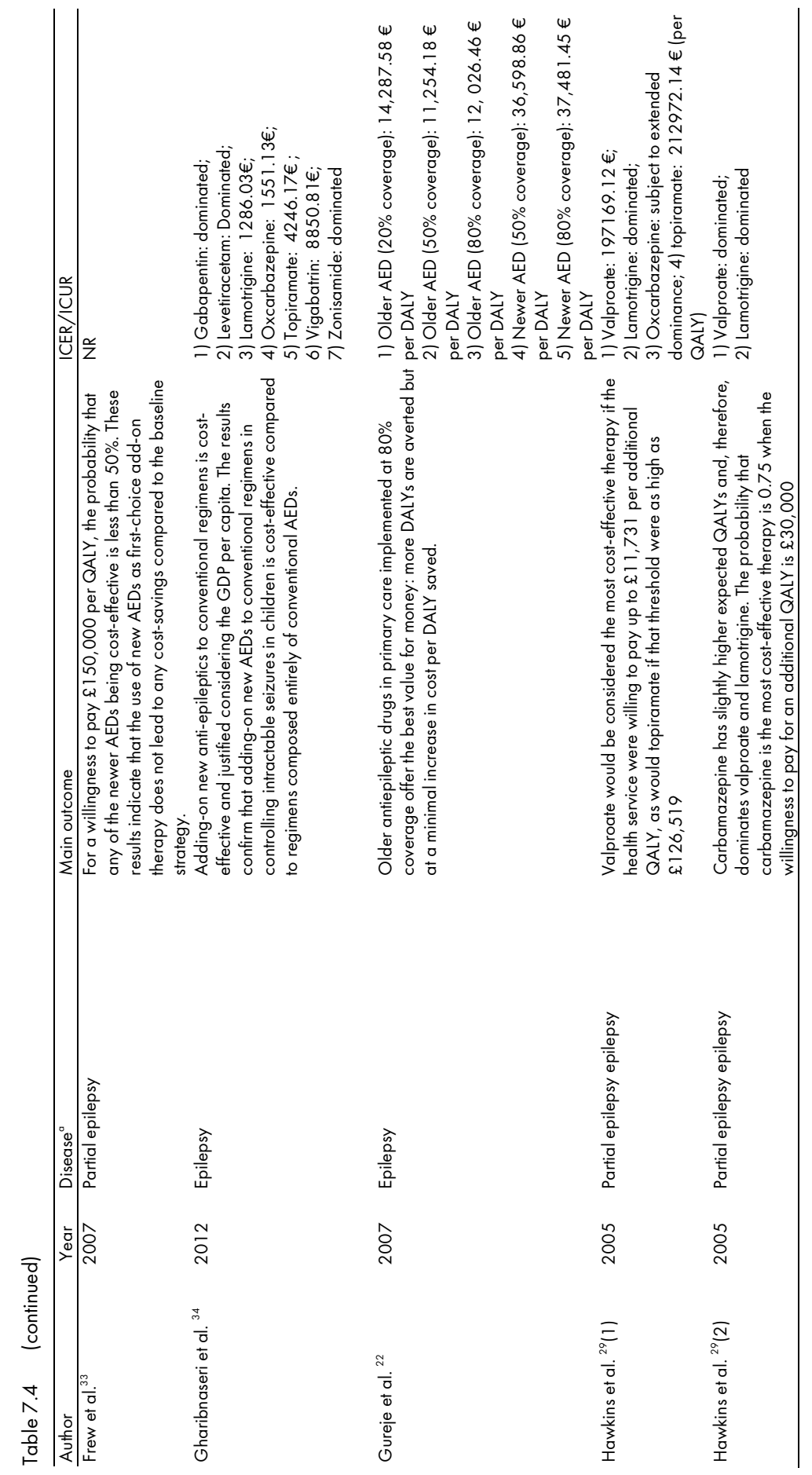




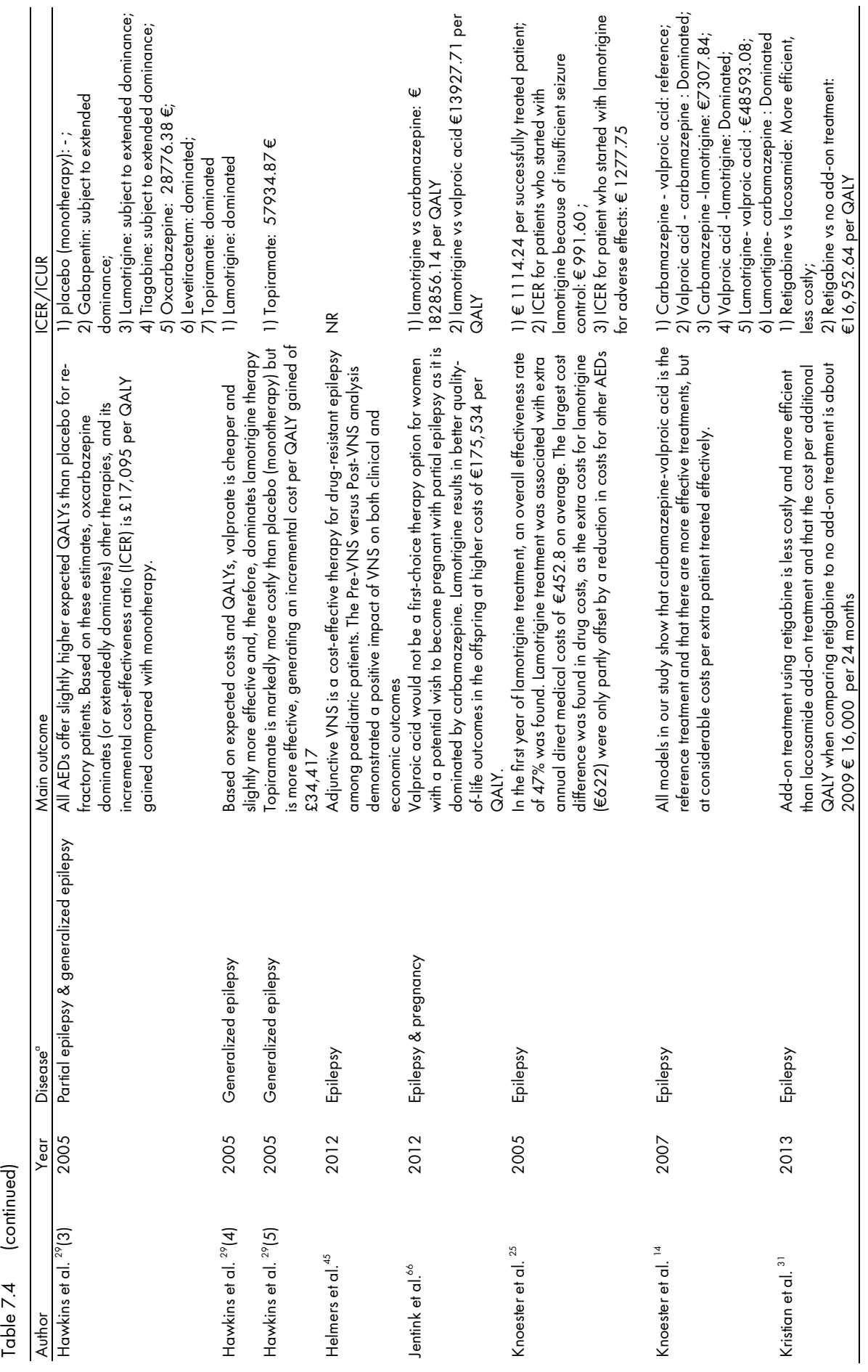




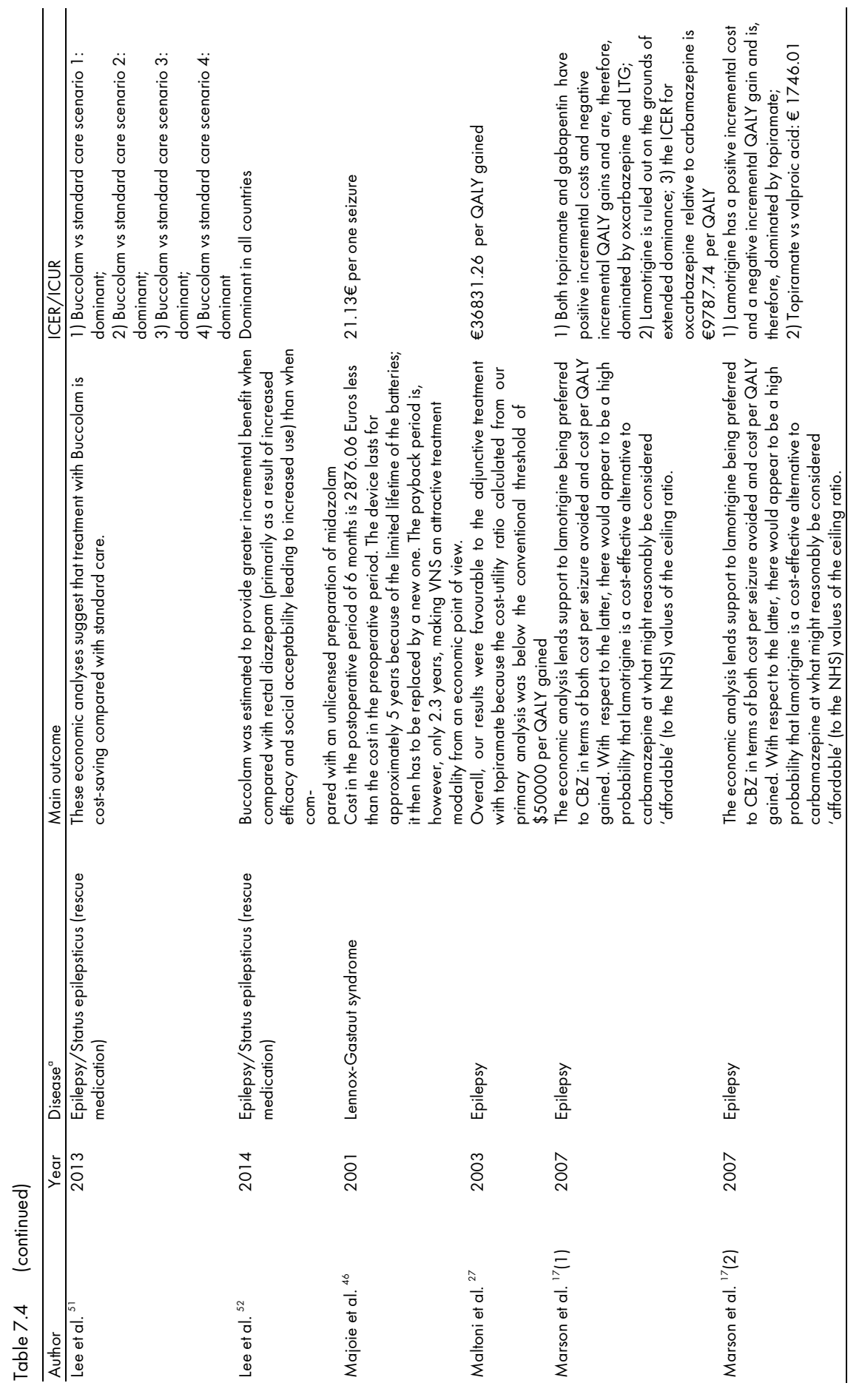




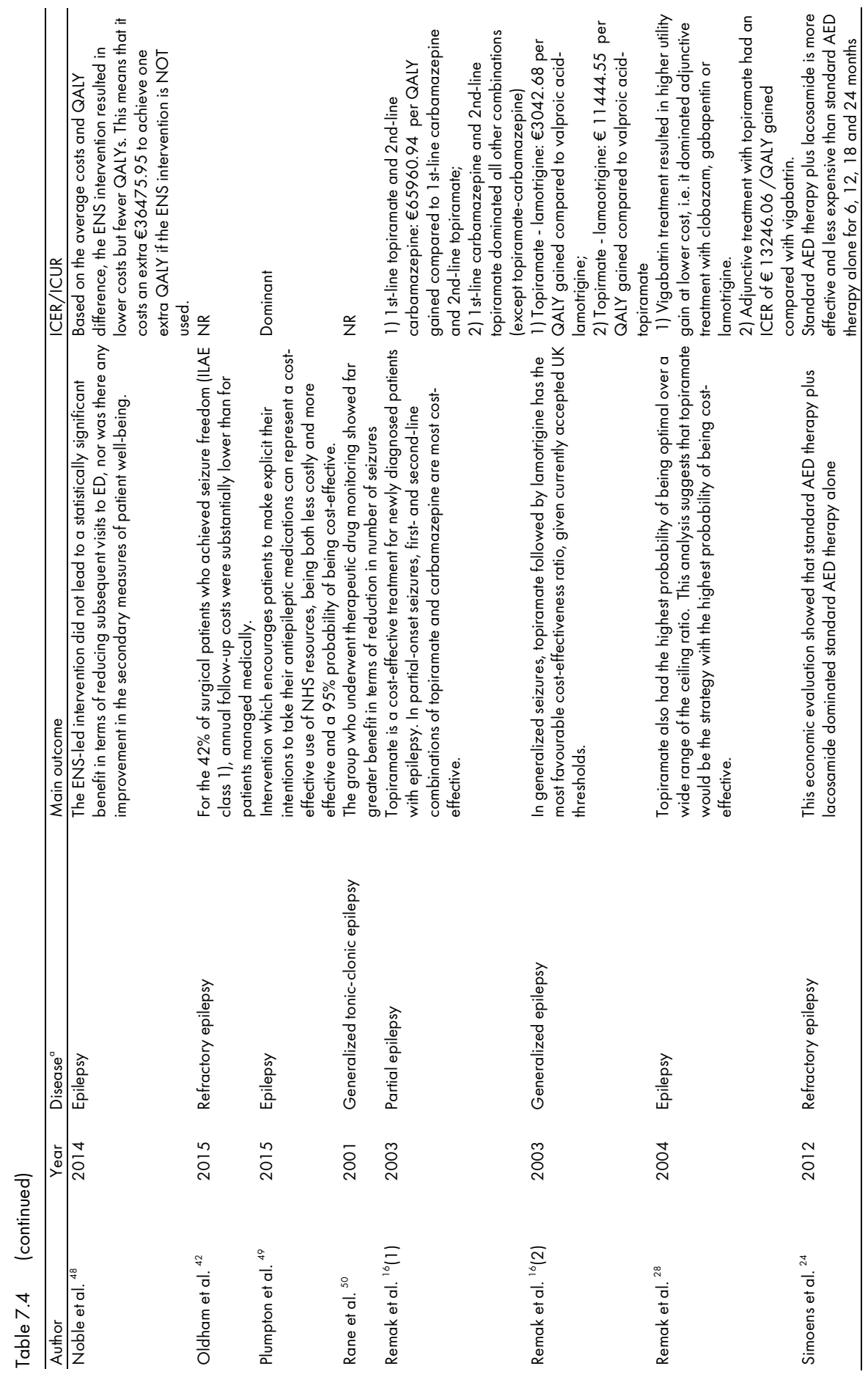




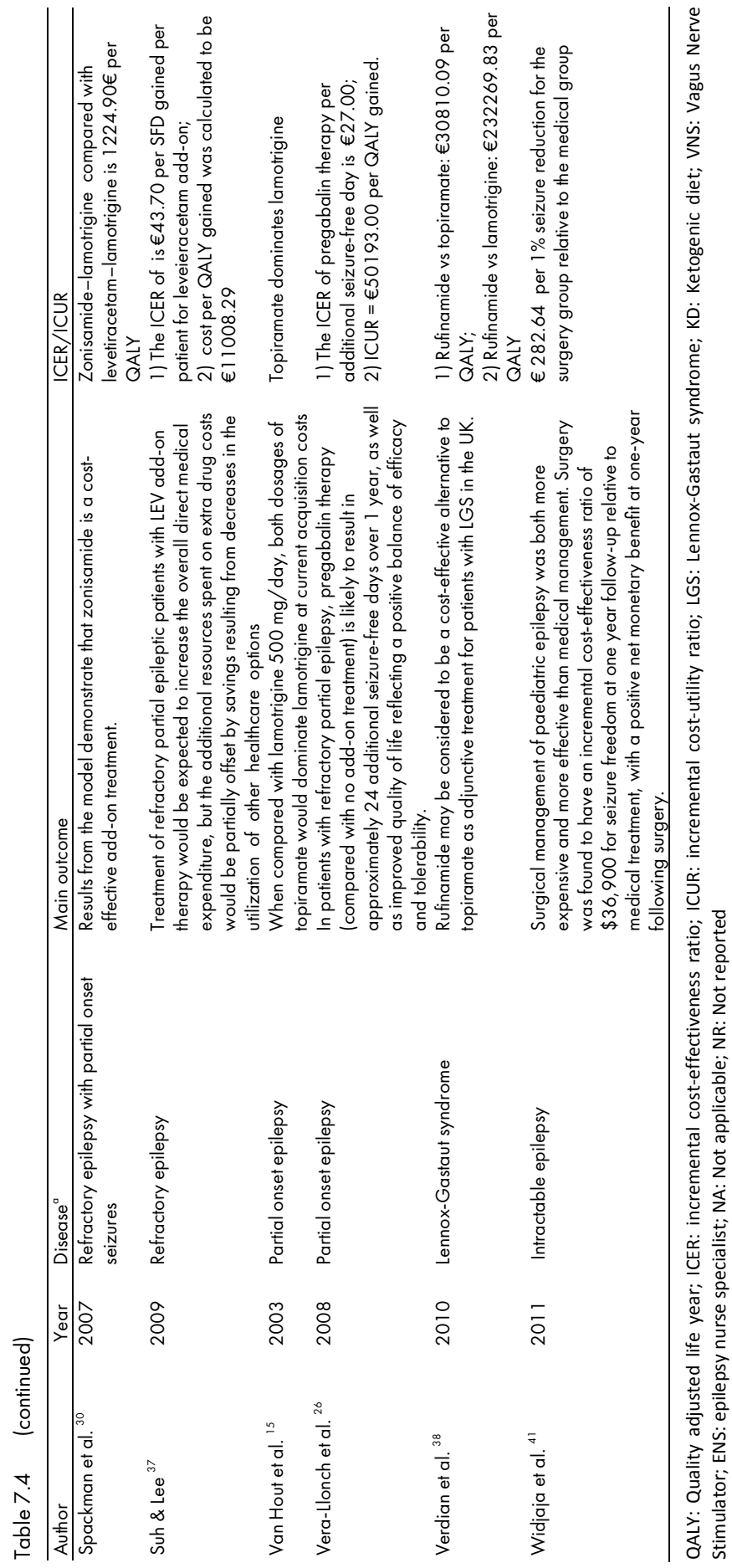


One study compared lamotrigine with topiramate. ${ }^{15}$ With regard to the ICER, it concluded that topiramate would dominate lamotrigine at current acquisition costs. Another study compared topiramate with lamotrigine and carbamazepine in patients with partial epilepsy, and with valproate and lamotrigine in patients with generalized epilepsy. ${ }^{16}$ In patients with partial epilepsy, 1 st-line topiramate and 2 nd- line carbamazepine resulted in an ICUR of $€ 65,961$ per QALY gained, compared to 1 st-line carbamazepine and 2 ndline topiramate; 1st-line carbamazepine and 2 nd-line topiramate dominated all other combinations. In patients with generalized epilepsy, topiramate followed by lamotrigine resulted in an ICUR of $€ 3,043$ per QALY gained, compared to valproate followed by lamotrigine; and topiramate followed by lamotrigine resulted in an ICUR of $€ 1$ 1,445 per QALY gained, compared to valproate followed by topiramate.

Marson et al. ${ }^{17}$ carried out two separate comparisons to examine the longer-term outcomes of standard versus new antiepileptic drugs. In the first comparison, they compared carbamazepine; gabapentin; lamotrigine; oxcarbazepine and topiramate. Topiramate and gabapentin were dominated by oxcarbazepine and lamotrigine. The ICUR for oxcarbazepine relative to carbamazepine was $€ 9,788$ per QALY gained. In the second comparison, they compared valproate, lamotrigine and topiramate and found that lamotrigine was dominated by topiramate and that the ICUR of topiramate compared to valproate was $€ 1,746$ per QALY gained.

Balabanov et al. ${ }^{18}$ have compared oxcarbazepine with carbamazepine but calculated only cost-effectiveness ratios (CERs). The CERs of carbamazepine (two different brands) were $€ 911$ and $€ 1,153$ per QALY gained and the CER of oxcarbazepine was $€ 2,761$ per QALY gained. In another study, Balabanov et al. ${ }^{19}$ compared patients on monotherapy with carbamazepine and valproate but did not calculate ICERs.

Lastly, Chisholm et al. $^{20}$ compared the use of phenobarbitone, phenytoin, carbamazepine, and valproic acid to no treatment in 9 developing regions across the world and concluded that the most cost-effective technology is first-line treatment in primary care with older AEDs such as phenobarbitone and phenytoin, because their expected population-level impact is similar to those of other first-line AEDs yet their acquisition costs are appreciably lower. In addition, two studies examined the costeffectiveness of older versus newer AEDs in developing countries. Chisholm et al. ${ }^{21}$ concluded that for Sub-Sahara Africa and South east Asia older AEDs dominated newer AEDs in primary care both at $50 \%$ or $80 \%$ coverage. Likewise, albeit not calculating incremental costs and effects, Gureje et al. ${ }^{22}$ concluded that older AEDs in primary care implemented at $80 \%$ coverage offer the best value for money as more DALYs were averted at a minimal increase in cost per DALY saved.

\subsubsection{AED as add-on treatment}

Most add-on AEDs were compared to standard therapy, other add-on AEDs or placebo. Two articles compared lacosamide add-on with standard therapy and calculated an ICUR. Bolin et al. ${ }^{23}$ found an ICUR of $€ 30,409$ per QALY gained and Simoens et al. ${ }^{24}$ demonstrated that standard AED therapy plus lacosamide dominated standard AED 
therapy. Another article compared Lamotrigine add-on with standard therapy and calculated an ICER of $€ 1,114$ per patient with $50 \%$ or more seizure reduction. ${ }^{25}$ There was one article comparing pregabalin add-on with standard therapy, calculating an ICUR of $€ 50,193$ per QALY and an ICER of $€ 27$ per seizure-free day. ${ }^{26}$ Maltoni \& Messori ${ }^{27}$ compared topiramate add-on with standard therapy calculating an ICUR of $€ 36,831$ per QALY gained (24); and Remak et al. ${ }^{28}$ compared topiramate add-on with clobazam, gabapentin, lamotrigine and vigabatrin; they calculated an ICUR of $€ 13,246$ per QALY gained for topiramate compared with vigabatrin (other AEDs were dominated).

Hawkins et al. ${ }^{29}$ compared topiramate for generalized seizures with placebo add-on and calculated an ICUR of $€ 57,935$ per QALY gained. Furthermore, they compared different AEDs with placebo add-on for partial seizures and calculated an ICUR for oxcarbazepine of $€ 28,776$ per QALY gained (other AEDs were dominated).

Spackman et al. included switching between alternative AEDs and calculated an ICUR of $€ 1,225$ per QALY when first-line add-on therapy with zonisamide is followed by second-line lamotrigine compared with first-line add-on therapy with levetiracetam followed by second line lamotrigine. ${ }^{30}$ The mean cost per QALY is, therefore, a weighted average of different treatments.

One study examined the cost-utility of retigabine as add-on treatment compared to lacosamide as add-on treatment or no add-on treatment in adult patients with refractory epilepsy. $^{31}$ It was found that retigabine dominated lacosamide and that the ICUR of retigabine vs no add-on treatment was $€ 16,953$ per QALY. A study by Craig et al. ${ }^{32}$ compared retigabine with eslicarbazepine acetate, lacosamide, pregabalin, tiagabine, zonisamine, and no treatment (i.e. maintenance therapy). Retigabine was dominated by pregabalin; assuming pregabalin had been tried but failed, retigabine was dominated by tiagabine. However, assuming both pregabalin and tiagabines had been tried and failed, retigabine had an ICUR of $€ 23,294$ per QALY gained.

Frew et al. ${ }^{33}$ examined the cost-effectiveness of newer drugs (i.e. lamotrigine, gabapentin, topiramate, and oxcarbazepine) as add-on therapy for children with focal epilepsies in which they compared the add-on treatment with the situation before the newer AED. They concluded that if there is a willingness to pay $£ 150,000$ per QALY, the probability of any of the newer AEDs being cost- effective is less than $50 \%$. Gharibnaseri et al. $^{34}$ examined the cost-effectiveness of adding-on new antiepileptic drugs to conventional regimens in an attempt to control intractable seizures in children. In this study, newer AED (i.e. gabapentin, levetiracetam, lamotrigine, oxcarbazepine, topiramate, vigabatrin, zonisamide) compared with older AED; it was concluded that gabapentin, levetiracetam, and zonisamide were dominated by older AED. Lamotrigine, oxcarbazepine, topiramate and vigabatrim were deemed to be cost-effective treatments with ICERs of respectively $€ 1,286 ; € 1,551 ; € 4,246$; and $€ 8,851$ per responder.

\subsubsection{AED as add-on treatment for Lennox-Gastaut syndrome}

Two articles have compared levetiracetam add-on with standard therapy in the treatment of patients with the Lennox-Gastaut syndrome. ${ }^{35,36}$ Compared with standard therapy, Blais 
et al. ${ }^{36}$ calculated an ICER for levetiracetam of $€ 80$ per seizure-free day gained and Suh \& Lee ${ }^{37}$ calculated an ICUR for levetiracetam of $€ 1$ 1,008 per QALY gained.

Two articles compared rufinamide add-on with lamotrigine or with topiramate addon. ${ }^{35,38}$ Benedict et al. ${ }^{35}$ calculated an ICER for rufinamide add-on of $€ 3,301$ per $1 \%$ increase in successfully treated patients with a seizure reduction of $50 \%$ or more, compared with lamotrigine add-on (topiramate was dominated). Verdian \& $\mathrm{Yi}^{38}$ calculated an ICUR for rufinamide add-on of $€ 30,810$ per QALY gained, compared with topiramate add-on (lamotrigine was dominated). Lastly, one study compared clobazam with rufinamide, topiramate and lamotrigine. At 3-month and 2-year follow-ups, clobazam dominated all other treatments compared. ${ }^{39}$

\subsection{Non-pharmacological interventions}

\subsection{Epilepsy surgery}

Three studies were identified which looked at the cost-effectiveness of epilepsy surgery and compared it with continued medical management, all in children with drug-refractory epilepsy. Bowen et al. ${ }^{40}$ evaluated 3 different scenarios, all with a time horizon of 20 years. Each of the analyses found that epilepsy surgery was cost-effective compared with continued medical management in children with drug-refractory epilepsy; the ICERs were $€ 52,961$ and $€ 19,079$ for 2 scenarios (starting the model at referral to a regional epilepsy centre and a district epilepsy centre, respectively). In the case of choosing epilepsy surgery versus continued medical management in children known to be suitable candidates for surgery, the epilepsy surgery was found to be less costly and provided greater clinical benefit (i.e., is a dominant strategy). In addition, Widjaja et al. ${ }^{41}$ found that surgical management of paediatric epilepsy was both more expensive and more effective than medical management. Surgery was found to have an incremental cost-effectiveness ratio of $€ 28,264$ for seizure freedom at one-year follow-up relative to medical treatment, with a positive net monetary benefit one-year after surgery. The study by Oldham et al. ${ }^{42}$, which did not calculate an ICER, found that in $42 \%$ of surgical patients who achieved seizure freedom (ILAE class 1), annual follow-up costs were substantially lower than for patients managed medically.

\subsubsection{Ketogenic diet}

Two articles were identified which looked at the cost-effectiveness of the ketogenic diet. $^{43,44}$ One study compared the ketogenic diet (KD) with the vagus nerve stimulator and care as usual (i.e. medication only) and concluded that from an economic viewpoint, neither vagus nerve stimulation nor ketogenic diet is a cost-effective option compared to care as usual at 12 months. In the long-term, the probability of vagus nerve stimulation being cost-effective increased to $51 \%$ at 5 years (at a ceiling ratio of $€ 80,000$ per QALY). The probability of ketogenic diet being cost-effective becomes $63 \%$ at 5 years when all patients are treated with the classical ketogenic diet, without hospitalization during 
ketogenic diet initiation. ${ }^{44}$ In the other study, the KD was compared to CAU with a followup of 4 months. It was concluded that the KD reduced seizure frequency but did not improve quality of life resulting in an unfavourable cost per QALY ratio. ${ }^{43}$

\subsubsection{Vagus nerve stimulator}

In addition to the above-mentioned paper looking at the KD versus vagus nerve stimulator (VNS) and CAU, two studies were identified which examined the cost-effectiveness of vagus nerve stimulation in children with epilepsy ${ }^{45}$ and children with a special form of epilepsy, Lennox-Gastaut syndrome. ${ }^{46}$ Both studies compared the periods before and afterVNS implantation. Despite calculating an ICER/ICUR, Helmers et al. ${ }^{45}$ concluded that adjunctive VNS is a cost-effective therapy for drug-resistant epilepsy among paediatric patients. In addition, the pre-VNS versus post-VNS analysis demonstrated a positive impact of VNS on both clinical and economic outcomes. The study by Majoie et al. ${ }^{46}$ demonstrated an ICER of $€ 21$ per single seizure. In addition, the authors concluded that the cost in the 6-month postoperative period is $€ 2876.06$ less than the cost in the preoperative period, and that the device will last for approximately 5 years because of the limited lifetime of the batteries. It then has to be replaced by a new one. The authors further calculate, however, that the payback period is 2.3 years, making VNS an attractive treatment modality from an economic point of view.

\subsubsection{Self-management interventions}

Three studies looked at the cost-effectiveness of self-management interventions in adults with epilepsy. Beretta et al. ${ }^{47}$ compared a standard educational plan with usual care only (i.e. medical management). The standardized educational plan, which consisted of one hour of counselling patients, was administered on admission and in the same form after 1 month, as a reminder. Despite calculating an ICER/ICUR, the authors conclude that a patient-tailored educational strategy is effective in reducing drug-related problems (particularly drug interactions) in epilepsy patients with chronic comorbidities, without adding significant monetary costs. The study by Noble et al. ${ }^{48}$ examined the costeffectiveness of a self-management programme for adults with epilepsy attending the emergency department. An epilepsy nurse specialist intervention programme (2 one-toone sessions delivered on an outpatient basis) was compared to care as usual. Based on the average costs and QALY difference, the self-management intervention resulted in lower costs but fewer QALYs. The calculated ICER in this study showed that it costs an extra $€ 36,475.95$ to achieve one extra QALY if the new intervention is NOT used. However, authors add that the study was underpowered to detect the hypothesized effect on emergency department use.

Lastly, Plumpton et al. ${ }^{49}$ conducted an economic evaluation of a behaviour-modifying intervention to enhance antiepileptic drug adherence, which consisted of a booklet of selfreport measures following a neurology appointment. In this study, the intervention was both less costly and more effective, and therefore dominated care as usual. 


\subsubsection{Therapeutic Drug-monitoring}

One study examined the effect of therapeutic drug-monitoring in adult patients with generalized tonic-clonic epilepsy. ${ }^{50}$ The intervention group was compared to a care as usual group. The study did not incorporate an ICER/ICUR, but demonstrated a benefit in terms of reduction in number of seizures, number of adverse events, better earning status and marital status compared with the group who had not undergone the intervention.

\subsection{Vulnerable groups}

Both the ILAE and NICE guidelines identify several vulnerable groups requiring special attention when providing care. These areas are: 1) patients with cognitive deficits; 2) elderly patients; and 3) the treatment of (non)convulsive status epilepticus in both children and adults. No economic evaluations were found which specifically focused on the first two categories, nor on the treatment of (non)convulsive status epilepticus in adults.

Lee et al. ${ }^{51,52}$ conducted two studies on the cost-effectiveness of licensed oromucosal midazolam for the treatment of children experiencing acute epileptic seizure. In the first study, licensed oromucosal midazolam was compared to standard care (unlicensed buccal midazolam \& rectal diazepam), unlicensed buccal midazolam and rectal diazepam. The ICUR of licensed oromucosal midazolam appeared to be dominant in all comparisons. ${ }^{51}$ In the second study, Lee et al. ${ }^{52}$ compared licensed oromucosal midazolam to the same comparators, placing the outcomes in a country-specific context for Scotland, Wales, Germany, Spain, France, Italy and Switzerland. Again, the ICURs of licensed oromucosal midazolam appeared to be dominant in all comparisons.

\section{Discussion}

This review aims to present an overview of published full economic evaluations of all health care interventions for patients with epilepsy. The chosen structure follows a categorization used by the ILAE and NICE, which will also be applied in the first part of this discussion.

It is important to distinguish between studies which have performed a cost-utility analysis and those which have performed a cost-effectiveness analysis. In the cost-utility analysis, outcomes are presented in cost per QALY gain; in cost-effectiveness analysis, outcomes are presented in cost per clinical outcome measure (e.g. seizure frequency). In most countries, contrary to cost-effectiveness outcomes, willingness to pay (WTP) thresholds are available for QALYs. For example, in the Netherlands, the WTP for a QALY ranges between $€ 20,000$ and $€ 80,000$. $^{53}$ Hence, if an ICUR is below this threshold, the intervention is considered to be cost-effective. For clinical outcomes, it is difficult to decide whether or not an intervention is cost-effective, as it is often not known how much society is willing to pay for such a unit of effect (e.g. it is not known how much society is willing to pay for $50 \%$ seizure reduction). In addition, it is also difficult to estimate the improvement in 
QALYs given a certain clinical improvement (e.g. mapping the clinical status of a patient to a utility score).

As well as comparability between studies, it is of utmost importance that the evaluations are of high quality as influential decisions (e.g. reimbursement decision) depend on these. Overall, the methodological quality was acceptable; however, some studies performed significantly worse than others. A study of Wilby et al. ${ }^{54}$ examined the quality of economic evaluations in epilepsy to develop an economic model (without a standardized questionnaire) and concluded that there were problems in studies basing conclusions on a small number of trials and using inappropriate assumptions to extrapolate beyond the length of time of the study. A study of Odenoletkova et al. ${ }^{11}$ examined the quality of therapeutic education interventions on the development and progression of type 2 diabetes in a similar way (e.g. using the $\mathrm{CHEC}$ ) and revealed similar scores compared to our study. However, none of their included articles had a perfect score. In contrast, Steenbergen et $\mathrm{al}^{55}$ examined the quality of economic evaluations in major depressive disorder and found that the included studies only scored a maximum of $53 \%$ on the CHEC-list, indicating that the quality of the studies was poor. Lastly, a study of Udsen et al. ${ }^{56}$ examined the quality of economic evaluations within chronic obstructive pulmonary disease and also concluded that the quality of the economic evidence was poor.

\section{AED as monotherapy \& AED as add-on treatment}

Difficulties arise when comparing studies. The lack of comparability (e.g. due to different scope, different AED being compared or specific types of epilepsy) in terms of treatments and/or comparators, means it is difficult to draw overall conclusion. One can conclude that, in comparison to other categories, AEDs have been substantially subjected to economic evaluations.

\section{Epilepsy surgery}

No study calculated ICURs for epilepsy surgery. Surgery was, however, found to be more effective in all studies, resulting in ICERs ranging from $€ 19,079$ to $€ 52,961$ for seizure freedom at one-year follow-up. All studies were conducted in children. In children known to be suitable candidates for surgery, epilepsy surgery was found to be less costly and provided greater clinical benefit (i.e., is a dominant strategy). The lack of cost-utility analyses makes it difficult to use these studies in policy decisions. Also, there appears to be a knowledge gap in the economic evaluation of epilepsy surgery in adults.

\section{Ketogenic diet}

The KD was shown to be clinically effective, especially in the RCT of de Kinderen et al.. ${ }^{43}$ It was concluded that the KD reduced seizure frequency but did not improve quality of life; therefore, unfavourable cost per QALY ratios resulted. One should, however, be aware of the limited follow-up of the RCT (four-month interim analysis). 


\section{Vagus nerve stimulator}

One author calculated an ICUR and concluded that the vagus nerve stimulator was not a cost-effective option compared to care as usual at 12 months. In the long-term, the probability of vagus nerve stimulator being cost-effective increased to $51 \%$ at 5 years (at a ceiling ratio of $€ 80.000$ per QALY). ${ }^{44}$ Two other articles did not calculate an ICUR, but concluded this was a cost-effective therapy for drug-resistant epilepsy among paediatric patients $^{45}$ and an attractive treatment modality from an economic point of view for paediatric patients with Lennox-Gastaut syndrome. ${ }^{46}$ Again, the lack of cost-utility analyses makes it difficult to use these studies in policy decisions.

\section{Self-management interventions}

Due to the large variability in self-management intervention, it is difficult to draw broad conclusions. One can, however, conclude that the likelihood of a self-management programme being cost-effective is totally dependent on, among others, the content and intensity of the intervention.

\section{Therapeutic drug-monitoring}

No ICER/ICUR was calculated but a benefit was demonstrated in terms of seizure reduction, better earning status and marital status. ${ }^{50}$

\section{Vulnerable groups}

Although several vulnerable groups can be identified when looking at the population which suffers from epilepsy, only two studies could be identified which looked specifically at children experiencing acute epileptic seizures. In both of these, licensed oromucosal midazolam dominated standard care (unlicensed buccal midazolam \& rectal diazepam)..$^{51,52}$ Studies focusing on other vulnerable groups, such as adults or children with epilepsy combined with cognitive deficits or physical handicaps, are lacking.

\section{Conclusions}

To the best of our knowledge, this review is the first to examine economic evaluations within epilepsy for all pharmacological and non-pharmacological treatment currently available. Two reviewers were used to select and extract the data, multiple databases were used for the identification of articles and a quality check was performed for all included studies. In addition, the study protocol was published in the PROSPERO database beforehand and the PRISMA guidelines were followed.

The fact that all included articles differed substantially in methodology hampered the attempt to combine the information regarding various interventions meaningfully. In addition to the difference between model-based and trial-based economic evaluations, there are many parameters of an economic evaluation which can vary widely from one 
study to the other. Part of this heterogeneity is unavoidable, because of the multiple choices available when defining parameters of economic evaluations (e.g. designs, outcome measures, patient samples, etc.). On the other hand, part can be avoided, simply by following standard economic research methods. (e.g. using the same measure of effectiveness, preferably QALYs or using the same comparators). To overcome this problem, the NICE institute adopted a decision-analytical approach in which all available evidence is synthesized into one newly developed economic model. Albeit being labourintensive, this approach increases the comparability between studies and makes it more feasible to reach overall (reimbursement) decisions. ${ }^{57}$

Furthermore, the heterogeneity between the studies may stress the need to define a reference case and to derive consensus on what constitutes "standard optimal care" in terms of best clinical practice that could serve as a comparator for studies in epilepsy in general and what the preferred clinical outcome measure would be (e.g. $50 \%$ seizure reduction, seizure-free patients, etc.). Differences were not only attributable to economic parameters but also clinical outcome measures varied extensively. A reference case is a set of methodological choices for a range of items relevant to conducting an economic evaluation that frame the boundaries of the study, such as model horizon, outcome measure(s), resource use and costing. ${ }^{58}$ Similar initiatives have been developed in the field of osteoporosis and osteoarthritis. ${ }^{58,59}$ A previous review on economic evaluations of AED in partial epilepsy also concluded that several methodological issues hampered their comparability, for example, the inclusion of productivity losses or time horizon. ${ }^{60}$ They also emphasized that future health economic evaluations would benefit from efficacy studies that compare relevant alternatives ${ }^{60}$ as there is a lack of head-to-head comparisons especially in AED studies.

The present study is likely to suffer from limitations, one being the lack of synthesized effectiveness data. This was, however, not possible given the wide differences between studies. Furthermore, this study only included published articles and did not search for conference proceedings. Hence, the results may be subject to a (minor) time lag.

Lastly, in this review we did not discriminate between studies that used a broad societal perspective and a more narrow or limited societal perspective. Studies claiming to have been conducted from a societal perspective have been identified as societal perspective irrespective of what costs or outcomes were included. However, studies who claimed to use a societal perspective but used a more narrow perspective (i.e. by omitting productivity losses without valid reasons) have been downgraded in their quality score, as some of the quality criteria specifically ask for the relation between the perspective and actual costs/outcomes included (e.g. items 8 and 11 of the CHEC-list and item 6 of the CHEERS checklist).

\section{Recommendations}

Results of this study are useful for policy makers or CPG developers. For example, they are likely to be used in the currently to be updated Dutch CPG for epilepsy. One should, 
however, take care to look at the quality of a study before taking the results for granted. Also, if one is interested in CPG development, other aspects, such as transferability of results, should be taken into account. Transferability is the extent to which the results of a study hold true for a different population or setting. ${ }^{61}$ For example, results from India may not be applicable to the Netherlands due to difference in standard practice or in disease prevalence. To obtain information regarding country-specific pharmacoeconomic guidelines, the International Society of Pharmacoeconomics and Outcomes Research (ISPOR) has developed a comparative table of 33 countries, including key features for several (mostly European \& American) countries (http://www.ispor.org/ peguidelines/index.asp). Furthermore, if one is interested in CPG development, it is important to keep updating this review. The results of several studies are expected to be published within one or two years (e.g. SANAD-II trial in England, self-management trials for people with epilepsy ${ }^{62,63}$, and the long-term results of De Kinderen et al. ${ }^{64}$ ).

Lastly, there is apparently only a small number of economic evaluations in certain areas such as vulnerable groups. Future research should aim to identify and fill knowledge gaps in specific areas within the field of epilepsy and economic evaluations. This review highlights the need for a more standardized approach for conducting economic evaluations within the field of epilepsy. One way forward would be the development of a reference case providing a framework for future studies, hopefully resulting in a more transparent and homogeneous way of conducting economic evaluations. In addition, studies examining the mapping of clinical outcome measures to utility scores could potentially provide an additional way of creating more homogeneous and comparable (i.e. to other disease areas) outcomes. For example, De Kinderen et al. ${ }^{65}$ have put first efforts in creating a utility-function for transforming clinically relevant epilepsy outcome measures into utility estimates.

\section{Key points}

- To the best of our knowledge, this review is the first to examine economic evaluations within epilepsy for all pharmacological and non-pharmacological treatment currently available

- A total of 40 publications were included in this review, twenty-nine (73\%) of which were articles about pharmacological interventions or anti-epileptic drugs (AED).

- Overall, the methodological quality of the studies was acceptable; however, some studies performed significantly worse than others.

- Articles differed substantially in methodology hampering the attempt to combine the information regarding various interventions in a meaningful way

- The heterogeneity between the studies stresses the need to define a reference case and to derive consensus on what constitutes "standard optimal care"

- The number of economic evaluations in certain areas, such as vulnerable groups, appears to be limited. Future research should aim to identify and fill knowledge gaps in specific areas within the field of epilepsy and economic evaluations. 


\section{Acknowledgements}

The work reported in this paper is part of a larger study supported by a grant from The Netherlands Organisation for Health Research and Development (ZonMW number: 171002204). We confirm that we have read the Journal's position on issues involved in ethical publication and affirm that this report is consistent with those guidelines. All coauthors have been substantially involved in the study and/or the preparation of the manuscript. The authors declare to have no conflict of interest. 


\section{References}

1. Van de Vel A, Cuppens K, Bonroy B, et al. Non-EEG seizure-detection systems and potential SUDEP prevention: State of the art. Seizure 2013;22:345-55.

2. Ngugi AK, Kariuki SM, Bottomley C, et al. Incidence of epilepsy: A systematic review and meta-analysis. Neurology $2011 ; 77: 1005-12$.

3. International Society For Pharmacoeconomics and Outcomes Research. Pharmacoeconomic Guidelines Around The World. Available at: http://www.ispor.org/peguidelines/index.asp2016.

4. Glauser T, Ben-Menachem E, Bourgeois B, et al. ILAE treatment guidelines: evidence-based analysis of antiepileptic drug efficacy and effectiveness as initial monotherapy for epileptic seizures and syndromes. Epilepsia 2006;47:1094-120.

5. Duncan JS, Sander JW, Sisodiya SM, et al. Adult epilepsy. Lancet 2006;367:1087-100.

6. Drummond MF, Sculpher MJ, Claxton K, et al. Methods for the economic evaluation of health care programmes. Oxford university press; 2015.

7. Brazier J. Measuring and valuing health benefits for economic evaluation. Oxford University Press; 2007.

8. Knies S, Severens JL, Ament AJHA, et al. The Transferability of Valuing Lost Productivity across Jurisdictions. Differences between National Pharmacoeconomic Guidelines. Value Health 2010;13:5 19-27.

9. Moher D, Liberati A, Tetzlaff J, et al. Preferred Reporting Items for Systematic Reviews and Meta-Analyses: The PRISMA Statement. PLoS Med 2009;6:e 1000097.

10. Evers $\mathrm{S}$, Goossens $M$, De Vet $\mathrm{H}$, et al. Criteria list for assessment of methodological quality of economic evaluations: Consensus on Health Economic Criteria. Int J Technol Assess Health Care 2005;21:240-5.

11. Odnoletkova I, Goderis G, Pil L, et al. Cost-effectiveness of therapeutic education to prevent the development and progression of type 2 diabetes: Systematic review. J Diab Metab 2014;2014.

12. Husereau $D$, Drummond $M$, Petrou $S$, et al. Consolidated health economic evaluation reporting standards (CHEERS) statement. Cost Eff Resour Alloc 2013;11:1.

13. International Monetary Fund. World Economic Outlook Database 2015. Available at: http://www.imf.org/external/pubs/ft/weo/2013/02/weodata/index.aspx/http://eppi.ioe.ac.uk/ costconversion/default.aspx.

14. Knoester PD, Deckers CL, Termeer EH, et al. A cost-effectiveness decision model for antiepileptic drug treatment in newly diagnosed epilepsy patients. Value Health 2007; 10:173-82.

15. Van Hout BA, Gagnon DD, McNulty P, et al. The cost effectiveness of two new antiepileptic therapies in the absence of direct comparative data: a first approximation. Pharmacoeconomics 2003;21:315-26

16. Remak E, Hutton J, Price M, et al. A Markov model of treatment of newly diagnosed epilepsy in the UK: an initial assessment of cost-effectiveness of topiramate. Eur J Health Econ 2003;4:271-8.

17. Marson AG, Appleton R, Baker GA, et al. A randomised controlled trial examining the longer-term outcomes of standard versus new antiepileptic drugs: the SANAD trial. Health Technol Assess 2007; 11 : $1-134$.

18. Balabanov PP, Zahariev ZI. A pharmacoeconomic comparison of monotherapy with Tegretol, Finlepsin and Trileptal (preliminary data). Folia Med (Plovdiv) 2006;48:37-43.

19. Balabanov PP, Zahariev ZI, Mateva NG. Evaluation of the factors affecting the quality of life and total costs in epilepsy patients on monotherapy with carbamazepine and valproate. Folia Med (Plovdiv) 2008;50:18-23.

20. Chisholm D. Cost-effectiveness of first-line antiepileptic drug treatments in the developing world: a population-level analysis. Epilepsia 2005;46:751-9.

21. Chisholm D, Saxena S. Cost effectiveness of strategies to combat neuropsychiatric conditions in subSaharan Africa and South East Asia: mathematical modelling study. BMJ 2012;344:e609.

22. Gureje $O$, Chisholm D, Kola L, et al. Cost-effectiveness of an essential mental health intervention package in Nigeria. World Psychiatry 2007;6:42-8.

23. Bolin K, Berggren F, Forsgren L. Lacosamide as treatment of epileptic seizures: cost utility results for Sweden. Acta Neurol Scand 2010;121:406-12.

24. Simoens S, De Naeyer L, Dedeken P. Cost effectiveness of lacosamide in the adjunctive treatment of patients with refractory focal epilepsy in Belgium. CNS Drugs 2012;26:337-50.

25. Knoester PD, Boendermaker AJ, Egberts AC, et al. Cost-effectiveness of add-on lamotrigine therapy in clinical practice. Epilepsy Res 2005;67:143-51. 
26. Vera-Llonch M, Brandenburg NA, Oster G. Cost-effectiveness of add-on therapy with pregabalin in patients with refractory partial epilepsy. Epilepsia 2008;49:431-7.

27. Maltoni S, Messori A. Lifetime cost-utility analysis of patients with refractory epilepsy treated with adjunctive topiramate therapy: cost-effectiveness in refractory epilepsy. Clin Drug Investig 2003;23:225-32.

28. Remak E, Hutton J, Selai CE, et al. A cost-utility analysis of adjunctive treatment with newer antiepileptic drugs in the UK. J Med Econ 2004;7:29-40.

29. Hawkins $N$, Epstein D, Drummond $M$, et al. Assessing the cost-effectiveness of new pharmaceuticals in epilepsy in adults: the results of a probabilistic decision model. Med Decis Making 2005;25:493-510.

30. Spackman DE, Yeates A, Rentz AM, et al. The cost effectiveness of zonisamide as adjunctive therapy in adult partial seizure epilepsy. J Med Econ 2007; 10:455-73.

31. Kristian B, Wachtmeister K, Stefan F, et al. Retigabine as add-on treatment of refractory epilepsy: a costutility study in a Swedish setting. Acta Neurol Scand 2013;127:419-26.

32. Craig D, Rice S, Paton F, et al. Retigabine for the adjunctive treatment of adults with partial-onset seizures in epilepsy with and without secondary generalization: a NICE single technology appraisal. Pharmacoeconomics 2013;31:101-10.

33. Frew EJ, Sandercock J, Whitehouse WP, et al. The cost-effectiveness of newer drugs as add-on therapy for children with focal epilepsies. Seizure 2007; 16:99-112.

34. Gharibnaseri Z, Kebriaeezadeh A, Nikfar S, et al. Cost-effectiveness of adding-on new antiepileptic drugs to conventional regimens in controlling intractable seizures in children. DARU 2012;20:17.

35. Benedict A, Verdian L, Maclaine $G$. The cost effectiveness of rufinamide in the treatment of Lennox-Gastaut syndrome in the UK. Pharmacoeconomics 2010;28:185-99.

36. Blais L, Sheehy O, St-Hilaire JM, et al. Economic evaluation of levetiracetam as an add-on therapy in patients with refractory epilepsy. Pharmacoeconomics 2005;23:493-503.

37. Suh GH, Lee SK. Economic evaluation of add-on levetiracetam for the treatment of refractory partial epilepsy in Korea. Psychiatry Investig 2009;6:185-93.

38. Verdian L, Yi Y. Cost-utility analysis of rufinamide versus topiramate and lamotrigine for the treatment of children with Lennox-Gastaut Syndrome in the United Kingdom. Seizure 2010;19:1-11.

39. Clements KM, Skornicki M, O'Sullivan AK. Cost-effectiveness analysis of antiepileptic drugs in the treatment of Lennox-Gastaut syndrome. Epilepsy Behav 2013;29:184-9.

40. Bowen JM, Snead OC, Chandra K, et al. Epilepsy care in Ontario: an economic analysis of increasing access to epilepsy surgery. Ont Health Technol Assess Ser 2012;12:1-41.

41. Widjaja E, Li B, Schinkel CD, et al. Cost-effectiveness of pediatric epilepsy surgery compared to medical treatment in children with intractable epilepsy. Epilepsy Res 201 1;94:61-8.

42. Oldham MS, Horn PS, Tsevat J, et al. Costs and Clinical Outcomes of Epilepsy Surgery in Children With Drug-Resistant Epilepsy. Pediatr Neurol 2015;53:216-20.

43. De Kinderen RJ, Lambrechts DA, Wijnen BF, et al. An economic evaluation of the ketogenic diet versus care as usual in children and adolescents with intractable epilepsy: An interim analysis. Epilepsia 2016;57: $41-50$.

44. De Kinderen RJ, Postulart D, Aldenkamp AP, et al. Cost-effectiveness of the ketogenic diet and vagus nerve stimulation for the treatment of children with intractable epilepsy. Epilepsy Res 2015;1 10:1 19-31.

45. Helmers SL, Duh MS, Guerin A, et al. Clinical outcomes, quality of life, and costs associated with implantation of vagus nerve stimulation therapy in pediatric patients with drug-resistant epilepsy. Eur J Paediatr Neurol 2012;16:449-58.

46. Majoie HJ, Berfelo MW, Aldenkamp AP, et al. Vagus nerve stimulation in children with therapy-resistant epilepsy diagnosed as Lennox-Gastaut syndrome: clinical results, neuropsychological effects, and costeffectiveness. J Clin Neurophysiol 2001;18:419-28.

47. Beretta S, Beghi E, Messina $P$, et al. Comprehensive educational plan for patients with epilepsy and comorbidity (EDU-COM): a pragmatic randomised trial. J Neurol Neurosurg Psychiatry 2014;85:889-94.

48. Noble AJ, McCrone P, Seed PT, et al. Clinical- and cost-effectiveness of a nurse led self-management intervention to reduce emergency visits by people with epilepsy. PLoS One 2014;9:e90789.

49. Plumpton $\mathrm{CO}$, Brown I, Reuber $\mathrm{M}$, et al. Economic evaluation of a behavior-modifying intervention to enhance antiepileptic drug adherence. Epilepsy Behav 2015;45:180-6.

50. Rane CT, Dalvi SS, Gogtay NJ, et al. A pharmacoeconomic analysis of the impact of therapeutic drug monitoring in adult patients with generalized tonic-clonic epilepsy. Br J Clin Pharmacol 200 1;52:193-5. 
51. Lee D, Gladwell D, Batty AJ, et al. The cost effectiveness of licensed oromucosal midazolam (Buccolam((R))) for the treatment of children experiencing acute epileptic seizures: an approach when trial evidence is limited. Paediatr Drugs 2013;15:151-62.

52. Lee DC, Gladwell D, Hatswell AJ, et al. A comparison of the cost-effectiveness of treatment of prolonged acute convulsive epileptic seizures in children across Europe. Health Econ Rev 2014;4:6.

53. Zorg RvdVe. Zinnige en duurzame zorg. In Editor $(E d)^{\wedge}(E d s)$ Book Zinnige en duurzame zorg, Zoetermeer; 2006.

54. Wilby J, Kainth A, Hawkins N, et al. Clinical effectiveness, tolerability and cost-effectiveness of newer drugs for epilepsy in adults: a systematic review and economic evaluation. Health Techn Assess 2005;9:172

55. van Steenbergen-Weijenburg KM, van der Feltz-Cornelis CM, Horn EK, et al. Cost-effectiveness of collaborative care for the treatment of major depressive disorder in primary care. A systematic review. BMC Health Serv Res 2010;10:1-10.

56. Udsen FW, Hejlesen O, Ehlers LH. A systematic review of the cost and cost-effectiveness of telehealth for patients suffering from chronic obstructive pulmonary disease. J Telemed Telecare 2014;20(4):212-20

57. Claxton K, Sculpher M, Drummond M. A rational framework for decision making by the National Institute for Clinical Excellence (NICE). Lancet 2002;360:71 1-5.

58. Hiligsmann M, Cooper C, Guillemin F, et al. A reference case for economic evaluations in osteoarthritis: An expert consensus article from the European Society for Clinical and Economic Aspects of Osteoporosis and Osteoarthritis (ESCEO). In Editor $(\mathrm{Ed})^{\wedge}(\mathrm{Eds})$ Book A reference case for economic evaluations in osteoarthritis: An expert consensus article from the European Society for Clinical and Economic Aspects of Osteoporosis and Osteoarthritis (ESCEO), Elsevier; 2014:271-82.

59. Hiligsmann M, Cooper C, Arden N, et al. Health economics in the field of osteoarthritis: an expert's consensus paper from the European Society for Clinical and Economic Aspects of Osteoporosis and Osteoarthritis (ESCEO). In Editor (Ed)^(Eds) Book Health economics in the field of osteoarthritis: an expert's consensus paper from the European Society for Clinical and Economic Aspects of Osteoporosis and Osteoarthritis (ESCEO), Elsevier; 2013:303-13.

60. Bolin K, Forsgren L. The cost effectiveness of newer epilepsy treatments: a review of the literature on partialonset seizures. Pharmacoeconomics 2012;30:903-23.

61. Knies S, Ament AJ, Evers SM, et al. The transferability of economic evaluations: Testing the model of Welte. Value in Health 2009; 12:730-738.

62. Krali-Hans I, Goldstein LH, Noble AJ, et al. Self-Management education for adults with poorly controlled epILEpsy (SMILE (UK)): a randomised controlled trial protocol. BMC Neurol 2014;14:69.

63. Leenen LA, Wijnen BF, de Kinderen RJ, et al. (Cost)-effectiveness of a multi-component intervention for adults with epilepsy: study protocol of a Dutch randomized controlled trial (ZMILE study). BMC Neurol $2014 ; 14: 255$.

64. De Kinderen RJ, Lambrechts DA, Postulart D, et al. Research into the (Cost-) effectiveness of the ketogenic diet among children and adolescents with intractable epilepsy: design of a randomized controlled trial. BMC Neurol 2011;11:1-10.

65. De Kinderen RJ, Wijnen BF, van Breukelen G, et al. From clinically relevant outcome measures to quality of life in epilepsy: A time trade-off study. Epilepsy Res 2016;125:24-31.

66. Jentink J, Boersma C, de Jong-van den Berg LT, et al. Economic evaluation of anti-epileptic drug therapies with specific focus on teratogenic outcomes. J Med Econ 2012;15:862-8. 


\section{Long-term clinical outcomes}

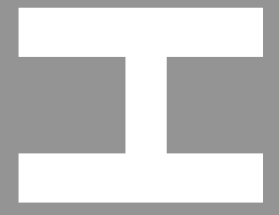

\section{and economic evaluation of}

the ketogenic diet versus

care as usual in children and adolescents with intractable epilepsy
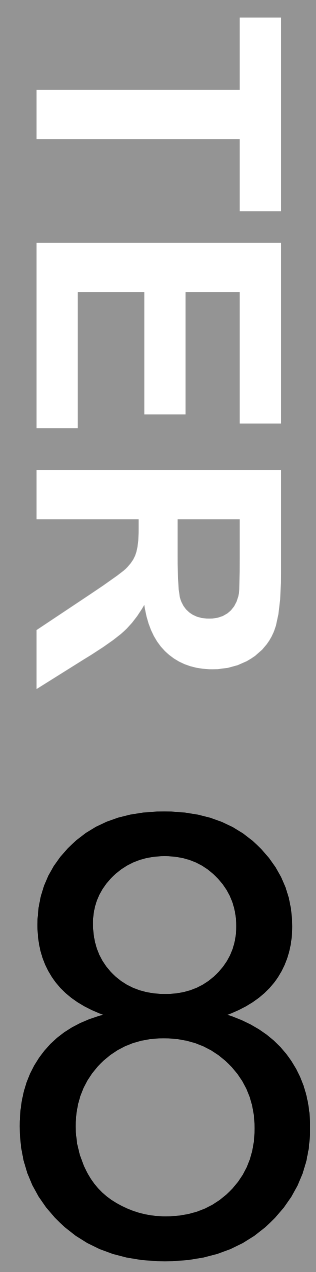

B.F.M. Wiinen R.J.A. de Kinderen D.A.J.E. Lambrechts D. Postulart B.A.P. Aldenkamp M.H.J.M. Majoie S.M.A.A. Evers Epilepsy Research 2017;137:91-99 


\section{Abstract}

\section{Objective}

To examine long-term retention rate, clinical outcomes, cost-utility and cost-effectiveness of the Ketogenic Diet (KD) compared with care as usual (CAU) in children and adolescents with intractable epilepsy from a societal perspective.

\section{Methods}

Participants were randomized into a KD or CAU group. Seizure frequency, quality adjusted life years (QALYs), side-effects, seizure severity, health care costs, production losses, patient and family costs were assessed at baseline and during 16-months of follow-up. Incremental cost-effectiveness ratios (ICERs) (i.e. cost per QALY and cost per responder) and costeffectiveness acceptability curves are presented.

\section{Results}

48 children were included in the analyses of this study (26 from KD group). In total, $58 \%$ of the KD group completed the follow-up of 16 months; 11 dropped-out for various reasons. At 16 months, $35 \%$ of the KD participants had a seizure reduction $\geq 50 \%$ from baseline, compared with $18 \%$ of the CAU participants. Mean costs per patient in the CAU group were $€ 53,367$ (extrapolated) compared to €61,019 per patient in the KD group, resulting in an ICER of $€ 46,564$ per responder. Cost per QALY rose well above any acceptable ceiling ratio. At 4months' follow-up, the KD group showed significantly more gastro-intestinal problems compared to the CAU group. At 16 months, the KD group reported fewer problems compared to CAU. Furthermore, $46.2 \%$ of the KD group reported a decrease in severity of their worst seizure compared to $32 \%$ of the CAU group.

\section{Conclusion}

The KD group resulted in more responders and showed greater improvement on seizure severity. Furthermore, the KD did not lead to worsening of side-effects other than gastrointestinal problems (only at 4 months' followup). However, as only a minimal difference in QALYs was found between the KD group and the CAU group, the resulting cost per QALY ratios were inconclusive. 


\section{Introduction}

Epilepsy imposes a substantial burden on individuals and society as a whole. ${ }^{1,2}$ In Europe, about 3.4 million people suffer from epilepsy, corresponding to a prevalence of 5 per 1,000 inhabitants. ${ }^{2}$ In the Netherlands, the health care costs of epilepsy in 2011 were $€ 248$ million accounting for $0.3 \%$ of the total budget. Kotsopoulos et al. examined ten epilepsy costs studies in different countries and found the proportion of national health care expenditure on epilepsy to be in the range $0.12-1.12 \%{ }^{3}$

Most patients with epilepsy are provided with an anti-epileptic drug (AED) treatment which is effective in about $70 \%$ of the treated patients. ${ }^{4}$ The remaining $30 \%$ patients who suffer from drug-resistant or intractable epilepsy are subjected to a wide range of possible treatments such as epilepsy surgery, vagus nerve stimulation, or deep brain stimulation to improve seizure control and hence the patient's quality of life (QoL). These procedures are, however, invasive and not always applicable for all patients with intractable epilepsy. Poorly controlled epilepsy results in frequent hospitalizations and consequently institutionalization in most cases in the Netherlands.

It has been suggested that the ketogenic diet (KD) can be beneficial to patients with intractable epilepsy. ${ }^{5}$ The KD is a high-fat diet with a restriction on carbohydrates that mimics the metabolic state of fasting. Ketone bodies replace glucose and become the main fuel for the brain's energy demands. Although the exact anticonvulsant mechanism of KD is unknown, children with drug-resistant epilepsy have been successfully treated with the KD since the 1920s. ${ }^{7}$ Moreover, there are many observational studies, ${ }^{8-15}$ reviews ${ }^{16-19}$ and two randomized controlled trials $(R C T)^{5,20}$ that suggest the beneficial effects of KD on seizure frequency. In addition, a recently published short-term interim analysis regarding the (cost-)effectiveness of the KD demonstrated a significant reduction in seizure frequency. ${ }^{21}$ None of the previous studies has, however, examined the long-term (costleffectiveness of the $\mathrm{KD},{ }^{18}$ which is important in order to make allocation or reimbursement decisions in health care, as policymakers often try to find a balance between costs and effects of interventions.

Hence, this study aims to examine long-term clinical outcomes, cost-utility and costeffectiveness of the KD compared with care as usual (CAU) in children and adolescents with intractable epilepsy from a societal perspective.

\section{Materials and methods}

\subsection{Design}

This economic evaluation was conducted alongside a randomized controlled trial. ${ }^{22}$ Subjects were randomized to either the ketogenic diet or to usual care. The total follow-up for the KD group was 16 months; the CAU group was followed for 4 months. A detailed description of the design of this study can be found elsewhere. ${ }^{23}$ 
Early in 2016, an interim analysis was published regarding the short-term results of this study. ${ }^{21}$ These results were based on the 4-month comparative phase of the study, after which subjects randomized to the KD were followed for an additional 12 months (with outcome assessments every 3 months). The results in this article are based on the total follow-up and compare findings from the KD group (16 months' follow-up) to the CAU group (4 months' follow-up, extrapolated to 16 months). Figure 8.1 is a graphical representation of the study. Analyses are based on the intention-to-treat protocol (ITT). This means that all patients initially randomized to either the KD or CAU group are included in the analyses. Study data of patients randomized to the control group were extrapolated to 16 months as it was assumed that they continued to receive care as usual and hence no sudden change in their health status would occur (for more details on extrapolation, see analysis section).

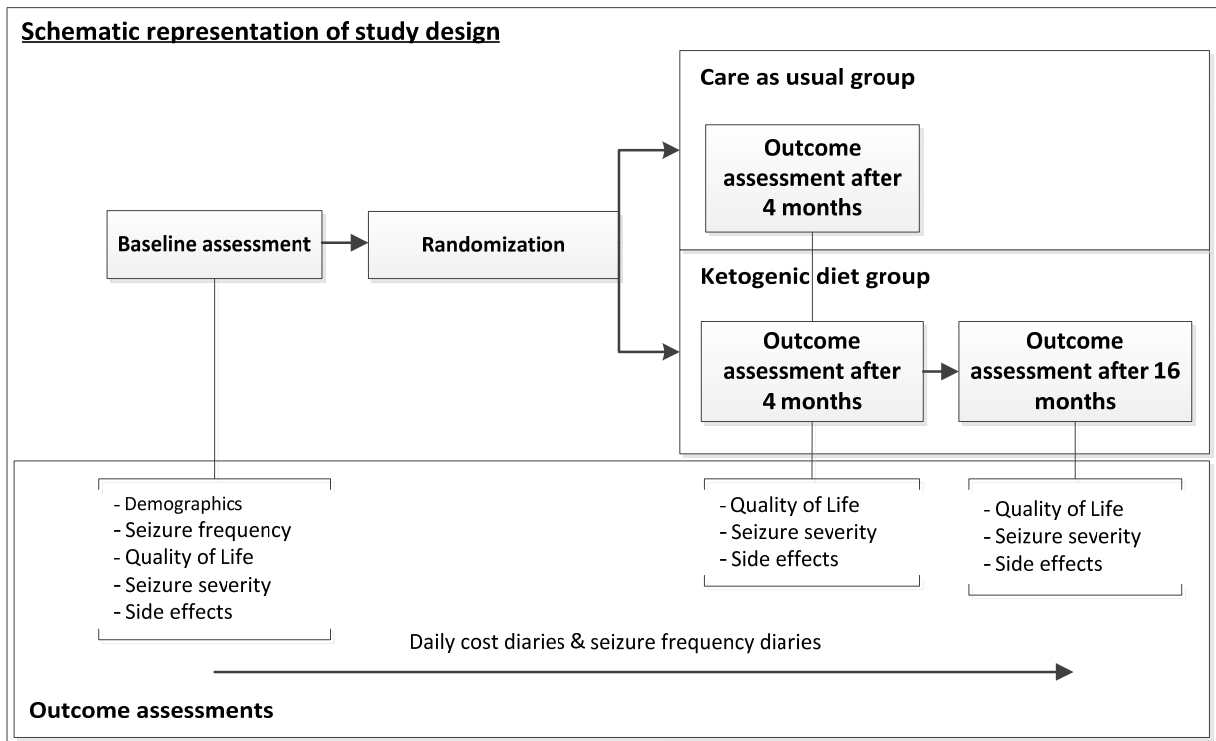

Figure 8.1 Overview of study design.

\subsection{Population \& setting}

This study included both children and adolescents with intractable epilepsy who had to meet the inclusion criteria: age between 1 and 18 years, diagnosed with intractable seizures (i.e. patients in whom at least 2 AEDs failed) and not eligible for epilepsy surgery, no fatty acid oxidation disorders or related diseases, no diabetes or hyperinsulinism, no prolonged QT-time syndrome, no hypercholesterolemia or hypertriglyceridemia, no severe liver, kidney or pancreas diseases, no renal tubular acidosis, no severe behavioral disorder, no malnutrition, no treatment with topiramate or acetazolamide and no positive 
family history of other risk factors for kidney stones or acidosis. Patients were included between July 2010 until August 2014. The study was conducted by the Academic Center of Epileptology Kempenhaeghe and has been approved by the ethics committee of the Academic Medical Center Utrecht, The Netherlands.

\subsection{Intervention}

Subsequent to the randomization, patients assigned to the KD group were admitted to the tertiary epilepsy center for a 5-day introduction to the KD. A dietician was in charge for both the introduction and consultation with the parents to determine whether the classical diet, the medium-chain triglyceride (MCT) diet, or a mixture of both diets should be introduced. When only tube feeding was given, a liquid form of the classical KD was used. Patients were instructed to continue taking their prescribed AEDs without change. During the 5-day administration of the KD, ketosis was assessed using two approaches: a daily assessment in urine (in case of those toilet-trained), and assessment in the blood by finger puncture on three occasions. The neurologist, pediatrician and epilepsy nurse also visited the children during this period. After the 5 days in the epilepsy center, parents were in charge of the ketosis check by daily assessment in urine or three times a week in blood (by finger puncture). An electrocardiogram was included in the protocol to screen for prolonged QT syndrome, which has been reported to be a possible side-effect of the KD. The progress of the patients was also tracked by weekly telephone meetings between parents and the epilepsy nurse, and between parents and the dietician. After 4 months, the dietician and epilepsy nurse continued to contact the parents (child) by e-mail or phone on a monthly basis. Furthermore, a visit to the neurologist, pediatrician, dietician and epilepsy nurse was scheduled every three months. Changes in AED regime were only allowed if medically necessary. No further protocol care was given. Outcomes were assessed at baseline and 4, 7, 10, 13 and 16 months after the baseline period.

\subsection{Care as usual}

Patients assigned to the control group (care as usual; CAU) were also instructed to continue taking their AEDs as prescribed and had weekly telephone meetings with the epilepsy nurse. 6 weeks and 4 months after the randomization, patients of the CAU group were seen by a neurologist, pediatrician and epilepsy nurse. Changes in AED regime were only allowed if medically necessary. No further protocol care was given. Outcomes were assessed at baseline and after 4 months. Given the design of the study, children in the CAU group were offered to receive the ketogenic diet with a delay of four months. 


\section{Outcome measures}

\subsection{Seizure frequency}

The seizure frequency was tracked by the use of a daily seizure calendar in which the types of seizures were labeled and described in accordance with the classification by the International League Against Epilepsy (ILAE). Patients with a mean seizure reduction of $50 \%$ or more (compared to the baseline) were labeled as responders. This cut-off was based on previous literature and was estimated to be a realistic clinical target for patients with intractable epilepsy. For this purpose, the 30-day baseline frequencies were compared to the 30-day frequency of the $16^{\text {th }}$ month. Patients in the KD group who dropped out were considered to be non-responders.

\subsection{Utilities}

The TNO-AZL Preschool Children's Quality of Life (TAPQOL) and Children's Quality of Life (TACQOL) were used to determine utilities for children aged between 1 and 5 years (parent proxy) and children aged between 6 and 16 years (parent proxy). The TAPQOL contains nine domains all ranging between 0 and 100. The TACQOL contains seven domains, two of which have scores ranging between 0 and 32 and two between 0 and 16. A mean score was calculated and transformed into a utility-like score between 0 and 1 in order to represent a utility. The EuroQol-Youth (EQ-5D-Y) was used to measure the utility of patients and the EuroQol 5-Dimensions (EQ-5D) was used to determine the utilities of parents. The EQ-5D health states were subsequently converted to a single utility score, which results in scores between 0 and 1 . Both the Dutch and the UK utility tariffs were applied to convert the health states of the EQ-5D into a utility. Utility scores from the EQ$5 D$ and utility-like utilities from the TAPQOL and TACQOL were measured at baseline, 4 months and 16 months. QALYs were calculated by means of the 'under the curve method', in which the time in a certain health state was multiplied by the utility of this health state. Effects were discounted with $1.5 \%$ for the period exceeding one year (last four months) according to the Dutch guidelines. ${ }^{24}$

\subsection{Seizure severity}

The National Hospital Seizure severity scale (NHS-3) was used to determine the severity of the patients' seizure. The NHS-3 consists of a structured interview in which the clinician rater assigns a score to seizure severity based on interference with patient function. Information is gathered from the patient and witnesses, if available. Eight questions are asked about: tonic-clonic movements, falling, injury, incontinence, altered consciousness, postictal impairment, and disruptive automatisms, generating a score in the range 1 to $27 .{ }^{25}$ A difference of 2 points was estimated to be clinically relevant change. The NHS-3 was assessed for the worst seizure type (if patients had different types of seizure) 
and for all seizure types together. Cases which were lost to follow-up were assumed to have no change in seizure severity.

\subsection{Side-effects}

Subjective symptoms were assessed using the Side-effects of Anti-Epileptic Drug questionnaire (SIDAED). ${ }^{26}$ The questionnaire was adapted to a parent-reported child version; items on sexuality were excluded. Nine domains of side-effects were evaluated: general central nervous system, behavior/irritability, depressive symptoms, cognitive functions, motor problems/coordination, vision, headache, cosmetic and dermatological problems, and gastrointestinal function. For each of the 43 items, the parents rate the severity of the symptom on a four-point Likert scale (no problem, mild, moderate, or serious problem). Patients who were lost to follow-up were excluded from the analyses as they no longer adhered to the KD.

\subsection{Costs}

Costs were calculated from a societal perspective; therefore, protocol-driven intervention costs, other health care costs, patient and family costs, and productivity losses of the parent(s) were measured. Costs were expressed in Euros and were converted (if necessary) to price year 2013 based on the relevant Consumer Price Index, to maintain comparability with the interim analysis. Costs were discounted with $4 \%$ for the period exceeding one year (last four months) according to the Dutch guidelines. ${ }^{24}$

Health care costs, patients and family costs, and other (epilepsy-related) costs, were measured by monthly cost diaries completed by patients' parent(s). Patient and family costs included formal (paid) care, informal care, and medical devices. Other costs included productivity losses of the parents, based on the hours absent from work due to their child's epilepsy or their own health-related problems.

Costs were evaluated using a bottom-up approach, in which volumes of each service used was multiplied by a standardized unit cost and summed to provide an overall total cost. Where possible, unit costs were based on standardized costs from the Dutch guidelines for cost research. Otherwise, real tariffs were used. Cost of medication was calculated using guideline prices, taking prescription charges for prescribed medication into account. When data on medication were diverse, lowest cost prices for the specific medication were used. The cost of informal care was calculated using shadow pricing. For this, the general hourly minimum wages were applied. Productivity costs were calculated on the basis of the Friction Cost Method (FCM), following the Dutch guidelines. ${ }^{24}$ The FCM is based on the assumption that an organization needs a certain time span (friction period) to replace the absent worker by another worker (for 2013, the friction period was 12 weeks). 


\subsection{Data analyses}

Data were analyzed using IBM SPSS Statistics version 23. Pearson chi-square test was used to determine whether groups differed regarding the number of responders at 4 months and 16 months. Independent sample t-tests were performed to determine whether the KD group differed from the CAU group with regard to the domain of the SIDAED.

Results from the CAU group, both costs and effects, at 4 months were extrapolated to 16 months using last observation carried forward. If data were totally absent, mean imputation per randomization group was used to impute missing Quality Adjusted Life Years (QALYs). For incomplete cases, last observation was carried forward.

Costs for KD group patients, for whom there was not even one follow-up visit after the comparative phase (i.e. a minimal follow-up of 7 months), were extrapolated using multiple imputations (5 times). Imputation was based on age, seizure frequency at 4 months, randomization group, diet type, and health care costs at 4 months. To account for non-normality of the cost data, predictive mean matching was used in which "real" observed values from similar cases were imputed instead of imputing regression estimates. $^{27,28}$

Baseline differences in costs and utilities between groups were checked with nonparametric bootstrapping, based on 1,000 bootstrap replications, as the data did not comply with the underlying assumptions of parametric tests. The arithmetic mean and $95 \%$ nonparametric confidence intervals were also calculated (1,000 bootstrap replications).

Furthermore, Microsoft Excel 2010 was used to quantify the uncertainty around the Incremental Cost Effectiveness Ratio (ICER; i.e. costs per responder) and the Incremental Cost Utility Ratio (ICUR; i.e. costs per QALY gained) by means of bootstrapping both the costs and effects $(5,000$ bootstrap replications). The ICERs/ICURs were calculated by dividing the incremental costs by the incremental effects (proportion of responders or QALYs). The ICERs/ICURs were presented on a cost-effectiveness (CE-)plane, which is divided into four quadrants (see Figures $8.2 \mathrm{a}$ and $8.2 \mathrm{~b}$ ). The north-east quadrant signifies a situation in which the alternative intervention is more costly and more effective, while the south-west quadrant signifies a situation in which the alternative intervention is less effective but also less costly. In these two quadrants, one needs to decide how much one is willing to pay (WTP) for an additional unit of effect (the threshold value). If an ICER/ICUR is below the threshold value, the intervention is considered to be cost-effective. There is currently no WTP value for an extra responder with $50 \%$ seizure reduction. There is, however, a (approximate) threshold for QALYs. In The Netherlands, there is an informal ceiling ratio between approximately $€ 20,000$ and $€ 80,000$ per QALY, depending on the burden of disease. ${ }^{29}$ For this study, a threshold of $€ 50,000$ was assumed, based on a disability weight of severe epilepsy of $0.657 .{ }^{30}$ To show the probability that a KD is cost-effective, given different ceiling ratios, a Cost-Effectiveness Acceptability Curve (CEAC) was constructed. This curve shows the percentage of ICURs that are below the threshold per ceiling ratio. 
A

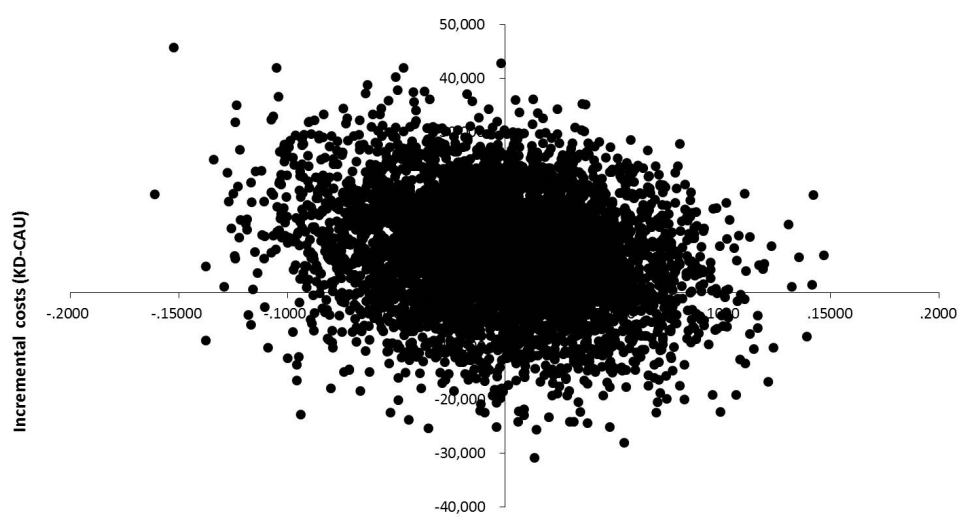

Incremental QALYs (KD-CAU)

B

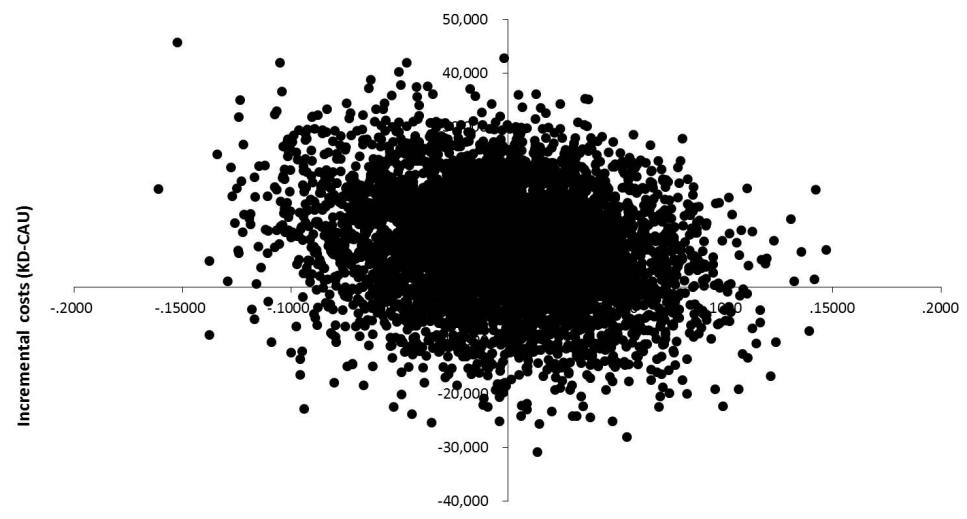

Incremental QALYS (KD-CAU)

Figure 8.2 a) Cost-effectiveness plane (costs per extra responder); b) Cost-effectiveness plane (costs per QALY gained).

Univariate sensitivity analyses were performed by taking a health care perspective compared to the societal perspective used in the base case. Furthermore, a sensitivity analysis on the valuation of the EQ-5D was performed, comparing the use of the Dutch tariff with the use of the UK tariff. Lastly, as hospitalization during the initiation of the KD is not common in all countries or hospitals, we have performed an incremental analysis in which the protocol driven costs of hospitalization were excluded from the intervention costs. 


\section{Results}

In total, 26 patients were included in the KD group and 22 patients in the CAU group. The mean ages were 7.8 and 8.1 years, respectively. The percentage of female patients was $30.8 \%$ in the KD group and $59.1 \%$ in the CAU group. In both groups almost all patients used more than one AED at the start of the study and had tried at least three different AEDs in the past. A detailed overview of the background characteristics can be found in Table 8.1.

Table 8.1 Demographic characteristics and baseline utilities.

\begin{tabular}{|c|c|c|c|}
\hline & & $\begin{array}{r}\text { Ketogenic diet } \\
(\mathrm{N}=26) \\
\mathrm{N}(\%)\end{array}$ & 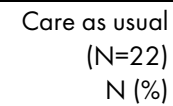 \\
\hline Female & & $8(30.8 \%)$ & $13(59.1 \%)$ \\
\hline Age mean (range) & & $7.8(2.10-16.5)$ & $8.1(1.1-15.7)$ \\
\hline Age seizure onset mean (range) & & $2.4(0-8)$ & $1.9(0-10)$ \\
\hline \multirow[t]{3}{*}{ Etiology } & Genetic & $9(34.6 \%)$ & $1(4.5 \%)$ \\
\hline & Structural & $2(7.7 \%)$ & $10(45.5 \%)$ \\
\hline & Unknown & $15(57.7 \%)$ & $11(50 \%)$ \\
\hline \multirow[t]{4}{*}{ Total IQ } & $<50$ & $10(38.5)$ & $11(50 \%)$ \\
\hline & $50-69$ & $3(11.5 \%)$ & $4(18.2 \%)$ \\
\hline & $70-99$ & $11(42.3 \%)$ & $5(22.7 \%)$ \\
\hline & $\geq 100$ & $2(7.7 \%)$ & $2(9.1 \%)$ \\
\hline \multirow[t]{3}{*}{ Behavioral/cognitive problems } & ADHD & 0 & $1(4.5 \%)$ \\
\hline & ADD & $1(3.8 \%)$ & $1(4.5 \%)$ \\
\hline & Autism & $3(11.5 \%)$ & $5(22.7 \%)$ \\
\hline \multirow[t]{6}{*}{ Number of AEDs at start of study } & None & 0 & $1(4.5 \%)$ \\
\hline & One & $3(11.5 \%)$ & $4(18.2 \%)$ \\
\hline & Two & $12(46.2 \%)$ & $8(36.4 \%)$ \\
\hline & Three & $9(34.6 \%)$ & $5(22.7 \%)$ \\
\hline & Four & $2(7.7 \%)$ & $2(9.1 \%)$ \\
\hline & Five & 0 & $2(9.1 \%)$ \\
\hline \multirow[t]{4}{*}{ Diet type } & MCT & $18(69.2 \%)$ & NA \\
\hline & Classical & $2(7.7 \%)$ & NA \\
\hline & Mixture & $1(3.8 \%)$ & NA \\
\hline & PGT & $6(23.1 \%)$ & $5(22.7 \%)$ \\
\hline \multirow[t]{7}{*}{ Number AEDs tried in the past } & Three & $3(11.5 \%)$ & $4(18.2 \%)$ \\
\hline & Four & $4(15.4 \%)$ & $5(22.7 \%)$ \\
\hline & Five & $9(34.6 \%)$ & $3(13.6 \%)$ \\
\hline & Six & $3(11.5 \%)$ & $3(13.6 \%)$ \\
\hline & Seven & $2(7.7 \%)$ & $1(4.5 \%)$ \\
\hline & Eight & $4(15.4 \%)$ & $2(9.1 \%)$ \\
\hline & Nine & $1(3.8 \%)$ & $4(18.2 \%)$ \\
\hline VNS in the past & & $1(3.8 \%)$ & $1(4.5 \%)$ \\
\hline Epilepsy surgery in the past & & $1(3.8 \%)$ & 0 \\
\hline
\end{tabular}

KD: ketogenic diet; CAU: care as usual; AEDs: antiepileptic drugs; ADHD: attention-deficit and hyperactivity disorder; ADD: attention-deficit disorder; VNS: vagus nerve stimulator; $M C T$ : medium-chain triglycerides; PGT: percutaneous gastrostomy tube. 


\subsection{Retention rate}

Seven patients from the KD group failed to complete at least one follow-up visit after 4 months. These patients had a follow-up of 2 months $(N=2), 3$ months $(N=1)$, and 4 months $(\mathrm{N}=4)$. The reasons for discontinuation after 4 months of the study were: problems with compliance $(N=1)$; ineffective $(N=1)$; ineffective combined with adverse effects $(N=1)$; adverse effects $(N=2)$; seizures shifted from night to day $(N=1)$; and stopped informed consent $(\mathrm{N}=1)$. During the 12-month follow-up phase, another 4 patients dropped-out. These patients had a follow-up of 7 months $(\mathrm{N}=2)$ and 10 months $(\mathrm{N}=2)$ and the reason for discontinuation were: problems with compliance $(N=2)$, ineffective $(N=1)$, and ineffective in combination with adverse effects $(N=1)$. In total, 15 of 26 patients $(58 \%)$ completed the follow-up of 16 months.

\subsection{Seizure frequency}

Based on intention-to-treat, the number of responders at 4 and 16 months, defined as a seizure reduction of $\geq 50 \%$ compared to baseline, was $50 \%$ and $34.6 \%$ in the KD group and $18.2 \%$ in the CAU group, assuming that all cases which were lost to follow-up were non-responders at 12 months (see Table 8.2). At 4 months, there was a significant difference between the two groups regarding the proportion of responders $(p=0.022)$; at 16 months' follow-up, the difference was no longer significant $(p=0.171)$.

\subsection{Utilities}

Measuring utilities with the EQ-5D-Y seemed only possible in a minority of patients in our sample. We, therefore, used the QoL scores of the TAPQOL and TACQOL lagedependent) to calculate QALYs for the patients. The mean utility of the patients at, 4 months' follow-up and 16 months' follow-up was 0.753, 0.748 and 0.750, respectively, for the KD group and $0.745,0.753,0.753$ for the CAU group baseline (based on TAPQOL and TACQOL). The total QALYs for the 16 months' follow-up in the two groups were 0.996 and 0.998 . Note that the maximum QALY over 16 months is 1.33 (calculated as: 1/12*16; one QALY represents one year in perfect health). The mean utilities for the parents at baseline, 4 months' follow-up and 16 months' follow-up were $0.875,0.853$ and 0.838 for the KD group (UK tariffs) and $0.808,0.791$ and 0.791 for the CAU group (see Table 8.2).

\subsection{NHS-3}

Results of the NHS-3 are shown in Table 8.2. At 16 months' follow-up, $46.2 \%$ of the patients in the KD group showed a decrease in seizure severity of their worst seizure compared to $31.8 \%$ in the CAU group. Furthermore, for all seizures combined, $50 \%$ in the KD group showed a decrease in seizure severity compared to $31.8 \%$ in the CAU group (see Table 8.2). 
Table 8.2 Number of responders, seizure severity, side-effect, QALYs (patients), and QALY (parents) per group

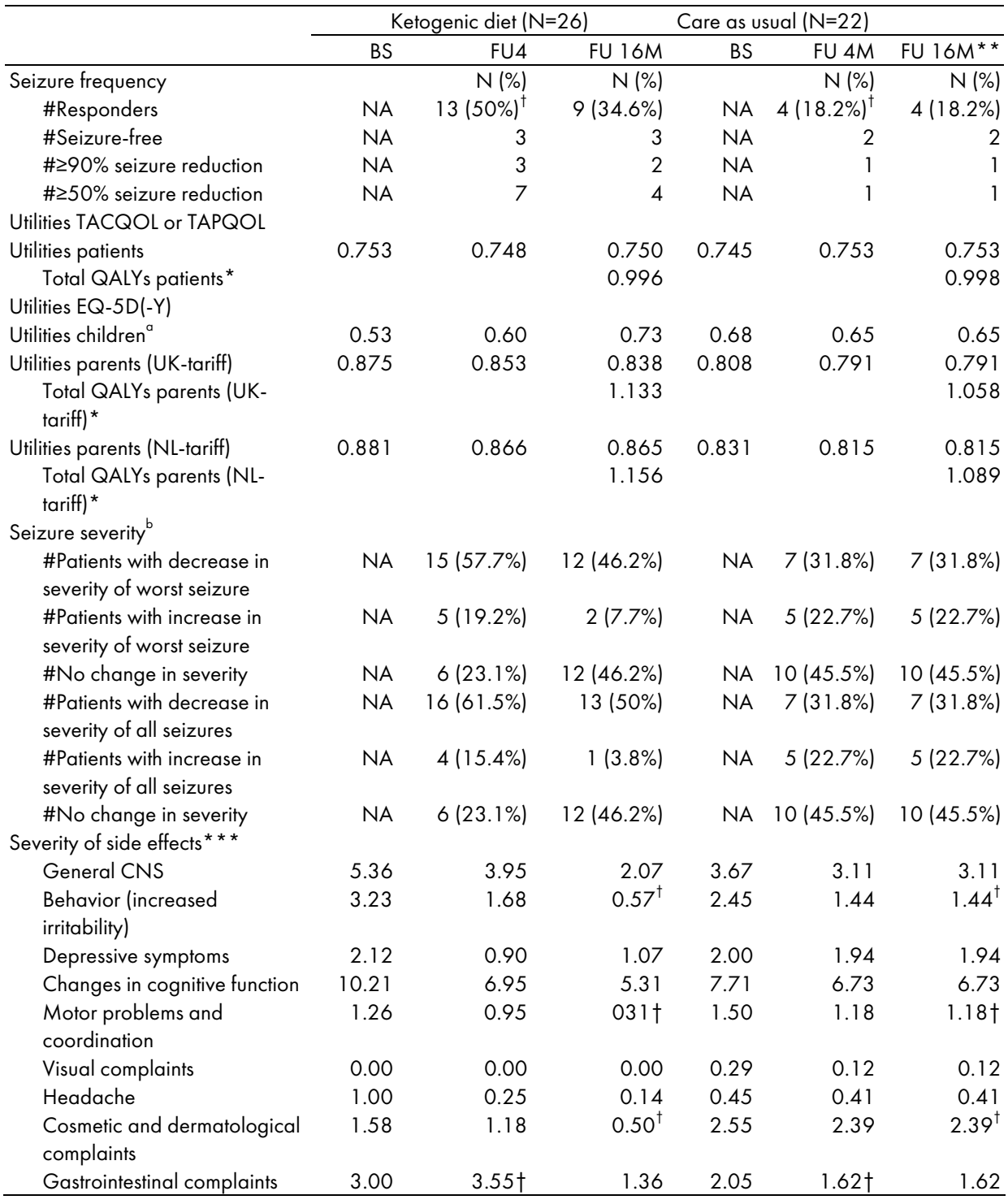

*Total QALYs are calculated over 16 months. The QALYs are, therefore, $(1 / 12)^{*} 16=1.33$; * Observations at 4 months carried forward; *** Patients who were lost to follow-up were excluded for this variable ( $N=9$ excluded in KD group); ${ }^{\dagger}$ : Sig. difference between groups at $5 \%$ level; ${ }^{a}$ The EQ-5D-Y was only measured in a few patients (from $N=11$ for $K D$ group at baseline and $N=11$ at baseline for the CAU group to $N=4$ at 12 months follow-up for the KD group); ${ }^{b}$ "The worst seizure" refers to the seizure type which has been identified by the patients as the worst type of seizure he/she has (in case one has multiple seizure types). "A// seizures" refers to the patients view on the severity of the overall seizure severity of his/her seizures. CNS: central nervous system; NA: not applicable; BS: baseline; FU: follow-up; 4M: 4 months; 16M: 16 months 


\subsection{Side-effects}

No significant difference was found between the groups at baseline. Only at 4 months, did patients in the KD group have significantly more symptoms related to gastro-intestinal problems $(p=0.000)$. After 16 months, patients in the KD group had significantly fewer side-effects with regard to behavior/irritability $(p=0.032)$, motor problems/coordination $(p=0.013)$, and cosmetic and dermatological problems $(p=0.001)$. No significant group differences were found in other domains (see Table 8.2).

\subsection{Incremental costs and effects}

The intervention costs were $€ 6,571$ for the $K D$ group and $€ 1,548$ for the control group (i.e. protocol-driven costs). The mean diet costs were $€ 9,483$, ranging from $€ 4,982$ for the mixed diet to $€ 13,906$ for the PGT diet. The costs for the classical diet were set to $€ 0$ as patients did not require any medicalized food to adhere to this diet. Total health care costs were $€ 34,180$ for the KD group and $€ 23,928$ for the control group. Patients and family costs, which mainly consist of informal care costs, were $€ 22,517$ and $€ 24,102$ for the KD and CAU groups, respectively; productivity losses accounted for $€ 4,275$ and $€ 5,337$, respectively in the KD and CAU groups (see Table 8.3). Taken together, these costs result in an ICER of $€ 46,564$ per responder. As there was no difference in QALYs between groups, costs per QALY (i.e. ICUR) rose well above any acceptable ceiling ratio. In Figure $8.2 a$ and $8.2 b$, the uncertainty around the base case ICER/ICUR is presented on the CEplane. Figure 8.3 represents the CEAC for the costs per QALY gained. At a maximum WTP (or threshold) of $€ 50,000$ per extra QALY, there is a $25 \%$ chance that the KD will be costeffective (see Figure 8.3). Using a health care perspective resulted in an ICER of $€ 207,990$ per responder. Excluding protocol-driven hospitalization costs for the admission of the KD resulted in an ICER of $€ 35,795$ per responder.

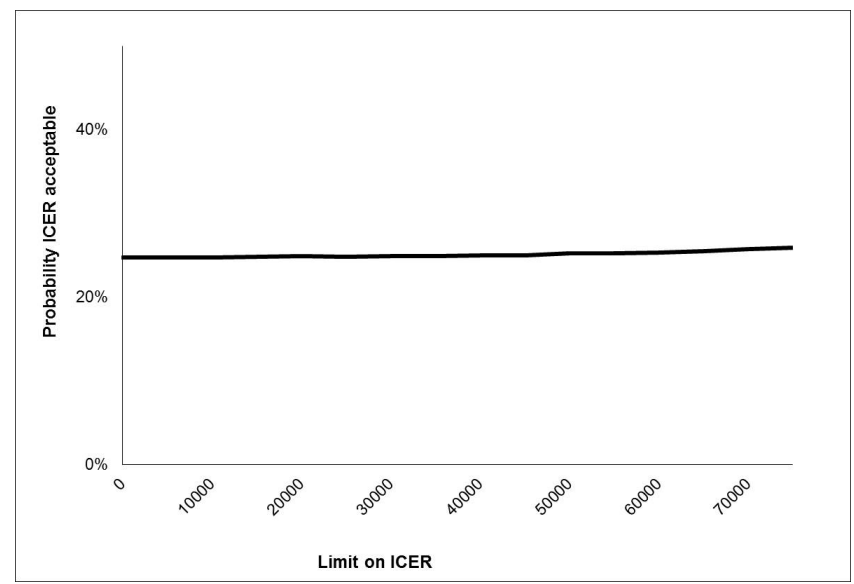

Figure 8.3 Cost-effectiveness acceptability curve (costs per QALY gained). 


\begin{tabular}{|c|c|c|c|c|c|c|}
\hline \multirow[b]{3}{*}{ Health care costs } & \multicolumn{3}{|c|}{ Ketogenic diet $(\mathrm{N}=19)$} & \multicolumn{3}{|c|}{ Care as usual $(\mathrm{N}=22)$} \\
\hline & \multicolumn{3}{|r|}{$95 \% \mathrm{Cl}$} & \multicolumn{3}{|c|}{$95 \% \mathrm{Cl}$} \\
\hline & & Lower bound & Upper bound & & Lower bound & Upper bound \\
\hline Intervention costs & 6571.31 & - & - & 1548.60 & - & - \\
\hline Hospitalization & 1769.77 & - & - & - & - & - \\
\hline Dietician & 529.09 & - & - & - & - & - \\
\hline Epilepsy nurse & 1535.17 & - & - & 986.92 & - & - \\
\hline Neurologist & 982.94 & - & - & 280.84 & - & - \\
\hline Pediatrician & 982.94 & - & - & 280.84 & - & - \\
\hline Ketosis check & 244.70 & - & - & - & - & - \\
\hline$E C G$ & 526.70 & - & - & - & - & - \\
\hline Diet costs & 9483.28 & 7702.48 & 11001.85 & & & \\
\hline MCT diet & 9964.80 & - & - & - & - & - \\
\hline Classical & 0.00 & - & - & - & - & - \\
\hline Mixture & 4982.40 & - & - & - & - & - \\
\hline Tube diet & 13905.60 & - & - & - & - & - \\
\hline GP visits & 93.36 & 42.73 & 147.38 & 53.91 & 17.42 & 101.99 \\
\hline Specialist visits & 1983.72 & 1214.27 & 2767.86 & 1402.40 & 873.70 & 2045.77 \\
\hline Paramedic visits & 2612.07 & 1367.23 & 4078.82 & 2781.86 & 1425.52 & 4494.27 \\
\hline Psychologist visits & 87.59 & 9.17 & 185.35 & 33.17 & 0.00 & 82.93 \\
\hline Emergency department & 50.20 & 0.00 & 166.18 & 0.00 & 0.00 & 0.00 \\
\hline General hospital & 2271.97 & 561.45 & 4677.18 & 2886.10 & 0.00 & 7125.05 \\
\hline Academic hospital & 320.00 & 56.47 & 630.60 & 238.43 & 0.00 & 715.30 \\
\hline Epilepsy center & 2369.18 & 87.68 & 6467.42 & 708.33 & 0.00 & 1898.33 \\
\hline Other in-patient stay & 3394.05 & 0.00 & 9403.96 & 0.00 & 0.00 & 0.00 \\
\hline Outpatient treaments & 4725.19 & 155.01 & 10228.38 & 12260.53 & 3854.29 & 22193.61 \\
\hline Social services & 332.71 & 30.26 & 1058.44 & 103.96 & 11.55 & 223.32 \\
\hline Prescribed medication & 1994.78 & 1417.27 & 2675.92 & 2152.20 & 1071.20 & 3622.73 \\
\hline Total health care costs ${ }^{*},{ }^{\star \star}$ & 34180.21 & 26278.11 & 42369.43 & 23927.80 & 14457.98 & 34532.33 \\
\hline \multicolumn{7}{|l|}{ Patient \& family costs } \\
\hline Informal care & 18540.55 & 11526.68 & 26630.98 & 19498.04 & 11296.15 & 27553.96 \\
\hline Child care ${ }^{\star \star \star}$ & 251.78 & 80.40 & 454.95 & 2262.61 & 368.78 & 5119.22 \\
\hline Home care & 3408.91 & 416.72 & 8173.11 & 2086.27 & 113.99 & 5562.27 \\
\hline Medication (OTC) & 74.79 & 17.39 & 161.39 & 76.16 & 0.00 & 223.80 \\
\hline Activities & 155.65 & 0.00 & 399.27 & 422.48 & 46.48 & 1074.37 \\
\hline Transport & 18.86 & 0.00 & 48.60 & 0.00 & 0.00 & 0.00 \\
\hline Total patient \& family costs ${ }^{*},{ }^{* *}$ & 22517.35 & 16049.5571 & 28605.07 & 24102.09 & 15238.71 & 33432.30 \\
\hline Production losses ${ }^{*},{ }^{\star \star}$ & 4275.29 & 2872.30 & 5869.82 & 5336.74 & 1690.36 & 9795.48 \\
\hline Total costs ${ }^{*},{ }^{* \star}$ & 61018.81 & 49242.04 & 72883.56 & 53366.63 & 35992.33 & 71360.65 \\
\hline
\end{tabular}

*For the KD/ intervention group: extrapolated to $\mathrm{N}=26$ based on age, seizure frequency, number of AEDs at start of study, health care costs at 4 months and randomization group, ${ }^{* \star}$ Last 4 months discounted with $4 \%$, OTC: Over the counter medication; ${ }^{* \star \star}$ This includes costs for special weekend or holiday placements/nursery for children \& adolescents

\section{Discussion}

This study was the first to examine the long-term (cost-)effectiveness of the KD in children with intractable epilepsy. The KD group resulted in more responders, but patients in this group were more bothered by side-effects related to the gastro-intestinal tract (only at 
4 months' follow-up). At 16 months' follow-up, patients in the KD group reported having significantly fewer side-effects regarding behavior/irritability, motor problems/ coordination, and cosmetic and dermatological problems. Furthermore, the KD group improved more on seizure severity, both in the worst seizure type as well as overall seizures. The costs of the total follow-up were higher in the KD group compared to the control group. However, as only a minimal difference was found between the KD and the CAU group regarding QALYs, inconclusive cost per QALY ratios resulted. Compliance with the KD was shown to be difficult in some patients, which is also reflected in the number of drop-outs. A retention rate of $58 \%$ is fairly low but in line with previous literature which demonstrated a retention rate of approximately $50 \%$ after 12 months' follow-up. ${ }^{31}$ The retention rate in this study at 12 months was $54 \%$. No difference was found in the quality of life of parents based on the EQ-5D. However, the domains of the EQ-5D may be too limited to have captured all relevant factors contributing to the increase of the quality of life of parents based on health state changes in their child.

The reduction in side-effects at 16 months' follow-up may be explained by several factors. First, the interim analysis already showed a decreasing trend in the number of symptoms related to gastro-intestinal problems from 6 weeks to 4 months' follow-up, probably due to small adjustments to the KD in consensus with the patients. Secondly, patients with severe side-effects may have dropped-out of the study given the retention rate of $58 \%$. Lastly, it is possible that the health state of patients on the KD may have improved and hence, they were less troubled by side-effects. We would like to stress, however, that these results should be interpreted carefully. At most, our results suggest that the KD did not lead to a worsening of side-effects other than gastro-intestinal problems.

In our study, most patients were given the MCT diet. However, a RCT by Neal et al. ${ }^{31}$ showed that the classical diet did not have any advantage over MCT diet in terms of efficacy and tolerability. Hence, we expect that this has not impacted the results.

The results reported in this article are similar to those presented in the interim analysis of this study (see ${ }^{21}$, in which the KD was also shown to improve clinical outcomes but failed to show benefits in terms of QALYs. However, this study provides broader insights in longterm clinical outcomes (such as side-effects and retention rate) and cost-effectiveness results.

To the best of our knowledge, this study and the interim analyses are the only trialbased economic evaluations of the KD in pediatric patients with intractable epilepsy. One model-based economic evaluation has been performed, which compared CAU, vagus nerve stimulation and the KD. It also concluded that, based on QALYs, the benefits of KD fail to outweigh the costs of the therapy. ${ }^{32}$ However, the authors state that the KD should not be ignored in the treatment of intractable epilepsy in individual or specific groups of patients. ${ }^{32}$ This statement can be substantiated by the (positive) clinical outcome found in the present study.

The lack of effect on quality of life might be explained by the fact that being a responder (or improvements on other outcome measures) is not enough to improve patient's quality of life. It is also possible, however, that the generic quality of life measures 
used in this study were not sensitive enough to detect changes in the patients' state of health. For example, it is suggested that targeted disease-specific or condition-specific utility instruments are likely to be more responsive than general or generic instruments. ${ }^{33}$

Other studies have examined the effectiveness of the KD in children with epilepsy and concluded that the KD should be considered as an alternative therapy for children with difficult-to-control seizures. ${ }^{10}$ The statement that the KD is a well-tolerated therapy for intractable pediatric epilepsy, ${ }^{10,34}$ may not be true for all patients, as was shown in this study.

The present study is subject to several limitations. First, the CAU group was only followed for 4 months and these results were extrapolated to 16 months. Hence, we assume that the 4-month measurements of the CAU group were sufficient to obtain a representative sample. The main reason was that it was perceived to be unethical to prolong the CAU period. Second, a large part of the KD group dropped-out of the study for various reasons resulting in a retention rate of $58 \%$. One factor contributing to this large drop-out may be the fact that the KD is relatively time and effort-intensive. Third, the use of the TACQOL and TAPQOL to derive utility estimates may be debatable, as the utility scores used to calculate QALYs are not preference-based utilities and consequently not comparable with other "real" utilities. At the start of the study, however, no suitable preference-based generic QoL instrument was available for utility measurements in children. Fourth, there appears to be marked differences in the etiology of epilepsy among the 2 groups. Specifically, in the KD group, a total of $34.6 \%$ of patients had genetically mediated epilepsy, $7.7 \%$ had a structural cause and $57.7 \%$ had an unknown etiology. In contrast, in the CAU group, $4.5 \%, 45.5 \%$, and $50 \%$ of patients had genetic, structural, unknown etiologies respectively. This may raise the possibility that etiology itself could be a contributing factor in the trial outcomes. Hence, a logistic regression analysis has been performed to examine whether there was any evidence regarding the influence of etiology on the change of being a responder in our study. In this model, the proportion of responders was taken as the dependent variable and gender, age, age at seizure onset, total intelligence quotient, syndrome classification, study group, and etiology as the dependent variables. Although the sample size was not estimated for the use of logistic regression, this analysis did not show a significant effect of etiology on the chance of being a responder in both groups. Fifth, in view of the nature of the treatments, blinding of the patients and researchers was not possible. This may have impacted our results to some extent. Lastly, transferability to other centers outside The Netherlands may be hampered by the fact that the study was conducted at a tertiary epilepsy center. For example, volumes and costs reported are (partly) related to the protocol of the tertiary epilepsy center Kempenhaeghe. Moreover, in our centre the KD is delivered in a more thorough way by admitting every patient to the hospital for 5-days, which is not commonly done in all hospitals/centers admitting the KD. Further studies may examine efforts to lower costs by de-intensifying the protocol. For example, prescribing the KD without 5-day admission to the epilepsy center, ignoring the telephone meetings with dietician and/or nurse, or by offering solely the classical variant of the diet. 


\section{Conclusions}

Given the positive clinical results of the KD compared to CAU demonstrated in this study, the KD may still be considered to be a potentially important treatment for children with intractable epilepsy. However, as there was no difference in quality of life between the two groups, at a maximum WTP of $€ 50,000$ per extra QALY, there is only a $25 \%$ chance that the KD will be cost-effective. Further research should focus on the relationship between quality of life and seizure frequency/seizure severity.

\section{Acknowledgments}

We would like to thank all the participants and their parents for taking part in this study. Furthermore, we would like to thank the neurologists, epilepsy nurses, dieticians, psychologists, pediatricians and other professionals for their contributions. This study was funded by the Netherlands Organization for Health Research and Development (ZonMw), grant application number 171002204. This study has been registered with The Netherlands Trial Registry (NTR2498). 


\section{References}

1. de Kinderen RJ, et al. From clinically relevant outcome measures to quality of life in epilepsy: A time tradeoff study. Epilepsy Res 2016;125:24-31.

2. Strzelczyk A, et al. Cost of epilepsy: a systematic review. Pharmacoeconomics 2008;26(6):463-76.

3. Kotsopoulos IA, et al. Estimating the costs of epilepsy: an international comparison of epilepsy cost studies. Epilepsia 2001;42(5):634-40.

4. Buncher CR, Tsay iy. Statistics in the pharmaceutical industry. 2nd ed. Statistics, textbooks and monographs. 1994, New York: M. Dekker. xviii, 563 p.

5. Neal EG, et al. The ketogenic diet for the treatment of childhood epilepsy: a randomised controlled trial. Lancet Neurol 2008;7(6):500-6.

6. Hartman AL, et al. The Neuropharmacology of the Ketogenic Diet. Pediatric Neurology 2007;36(5): 281-92.

7. Bromfield E, Carvazos J, Sirven J. An Introduction to Epilepsy. 2006: American Epilepsy Society.

8. Caraballo, R.H., et al., Ketogenic diet in patients with Dravet syndrome. Epilepsia 2005;46(9): 1539-44.

9. Coppola $G$, et al. The ketogenic diet in children, adolescents and young adults with refractory epilepsy: an Italian multicentric experience. Epilepsy Res 2002;48(3):221-7.

10. Freeman JM, et al. The efficacy of the ketogenic diet-1998: a prospective evaluation of intervention in 150 children. Pediatrics 1998; 102(6):1358-63.

11. Kang HC, et al. Efficacy and safety of the ketogenic diet for intractable childhood epilepsy: Korean multicentric experience. Epilepsia 2005;46(2):272-9.

12. Kankirawatana $P$, et al. Ketogenic diet: an alternative treatment for refractory epilepsy in children. J Med Assoc Thai 2001;84(7):1027-32.

13. Kossoff EH, et al. Efficacy of the ketogenic diet for infantile spasms. Pediatrics 2002; 109(5):780-3.

14. Kossoff EH, et al. Tuberous sclerosis complex and the ketogenic diet. Epilepsia 2005;46(10): 1684-6.

15. Vining EP, et al. A multicenter study of the efficacy of the ketogenic diet. Arch Neurol 1998;55(11): 1433-7.

16. Keene DL. A systematic review of the use of the ketogenic diet in childhood epilepsy. Pediatr Neurol, 2006;35(1):1-5.

17. Lefevre F, Aronson N. Ketogenic diet for the treatment of refractory epilepsy in children: A systematic review of efficacy. Pediatrics 2000; 105(4):E46.

18. Levy R, Cooper P. Ketogenic diet for epilepsy. Cochrane Database Syst Rev, 2003(3):Cd001903.

19. Sinha SR, Kossoff EH. The ketogenic diet. Neurologist 2005;11(3):161-70.

20. Sharma $S$, et al. Use of the modified Atkins diet for treatment of refractory childhood epilepsy: a randomized controlled trial. Epilepsia 2013;54(3):481-6.

21. de Kinderen RJ, et al. An economic evaluation of the ketogenic diet versus care as usual in children and adolescents with intractable epilepsy: An interim analysis. Epilepsia 2016;57(1):41-50.

22. Lambrechts DA, et al. A randomized controlled trial of the ketogenic diet in refractory childhood epilepsy. Acta Neurol Scand, 2016.

23. de Kinderen RJ, et al. Research into the (Cost-) effectiveness of the ketogenic diet among children and adolescents with intractable epilepsy: design of a randomized controlled trial. BMC Neurology 2011 ; $11(1): 1-10$.

24. Dutch Healthcare Institute, Richtlijn voor het uitvoeren van economische evaluaties in de gezondheidszorg. Diemen: Zorginstituut Nederland, 2015.

25. O'Donoghue MF, Duncan JS, Sander JW. The National Hospital Seizure Severity Scale: a further development of the Chalfont Seizure Severity Scale. Epilepsia 1996;37(6):563-71.

26. Uij S, et al. A cross-sectional study of subjective complaints in patients with epilepsy who seem to be wellcontrolled with anti-epileptic drugs. Seizure 2006; 15(4):242-8.

27. Grittner $U$, et al. Missing value imputation in longitudinal measures of alcohol consumption. Int J Methods Psychiatr Res $2011 ; 20(1): 50-61$.

28. Horton NJ, Lipsitz SR. Multiple imputation in practice: comparison of software packages for regression models with missing variables. The American Statistician, 2001;55(3):244-54.

29. Council for Public Health and Health Care, Sensible and sustainable care (in Dutch). 2006: Zoetermeer. 
30. Salomon JA, et al. Common values in assessing health outcomes from disease and injury: disability weights measurement study for the Global Burden of Disease Study 2010. Lancet 2013;380(9859):2129-43.

31. Neal EG, et al. A randomized trial of classical and medium-chain triglyceride ketogenic diets in the treatment of childhood epilepsy. Epilepsia 2009;50(5):1 109-17.

32. de Kinderen RJ, et al. Cost-effectiveness of the ketogenic diet and vagus nerve stimulation for the treatment of children with intractable epilepsy. Epilepsy Res 2015;110:119-31.

33. Wiebe $\mathrm{S}$, et al. Comparative responsiveness of generic and specific quality-of-life instruments. J Clin Epidemiol 2003;56(1):52-60.

34. Kossoff EH. A modified Atkins diet is effective for the treatment of intractable pediatric epilepsy. Epilepsia, $2006 ; 47(2): 421-4$ 



\title{
(Cost)-Effectiveness of a
}

\author{
Multi-component
}

Intervention for Adults

\author{
with Epilepsy
}

Study protocol of a Dutch randomized

controlled trial (ZMILE study)
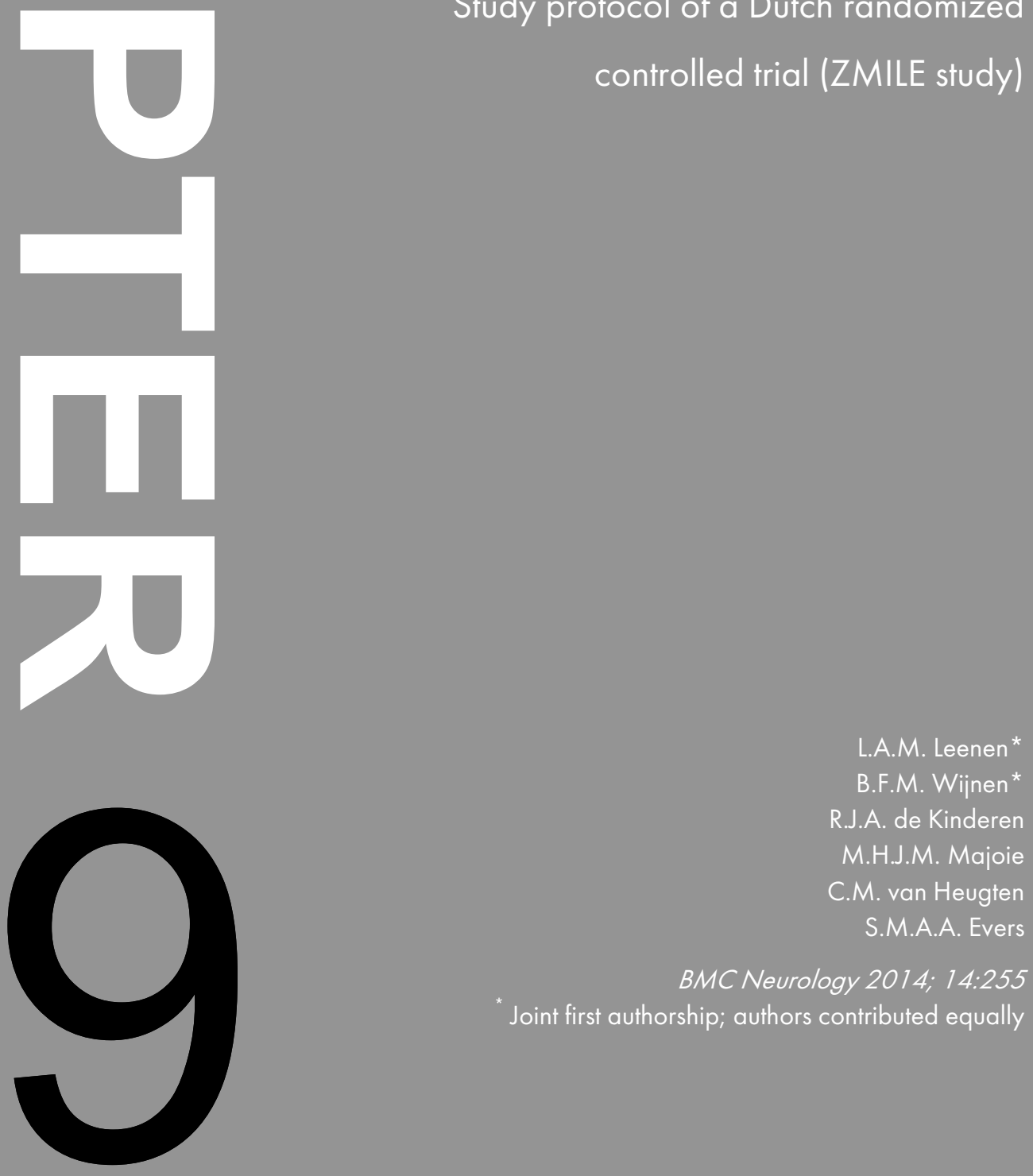


\section{Abstract}

\section{Background}

In patients with epilepsy, poor adherence to anti-epileptic drugs has been shown to be the most important cause of poorly controlled epilepsy. Furthermore, it has been noted that the quality of life among patients with epilepsy can be improved by counseling and treatments aimed at increasing their self-efficacy and concordance, thus stimulating self-management skills. However, there is a need for evidence on the effectiveness of such programs, especially within epilepsy care. Therefore, we have developed a multi-component intervention (MCl) which combines a selfmanagement/education program with e-Health interventions. Accordingly, the overall objective of this study is to assess the (cost)-effectiveness and feasibility of the $\mathrm{MCl}$, aiming to improve self-efficacy and concordance in patients with epilepsy.

\section{Methods}

A randomized controlled trial in two parallel groups will be conducted to compare the $\mathrm{MCl}$ with a control (care as usual) condition in epilepsy patients. One hundred eligible epilepsy patients will be recruited and allocated to either the intervention or control group. The intervention group will receive the $\mathrm{MCl}$ consisting of a self-management/education program of six meetings, including e-Health interventions, and will be followed for 12 months. The control group will receive care as usual and will be followed for 6 months, after which patients will be offered the possibility of participating in the $\mathrm{MCl}$. The study will consist of three parts: 1) a clinical effectiveness study, 2) a cost-effectiveness study, and 3) process evaluation. The primary outcome will be selfefficacy. Secondary outcomes include adherence, side effects, change in seizure severity \& frequency, improved quality of life, proactive coping, and societal costs. Outcome assessments will be done using questionnaires at baseline and after 3, 6, 9, and 12 months (last two applicable only for the intervention group).

\section{Discussion}

In times of budget constraints, $\mathrm{MCl}$ could be a valuable addition to the current healthcare provision for epilepsy, as it is expected that higher concordance and self-efficacy will result in reduced use of healthcare resources and an increased QOL. Accordingly, this study is aimed helping patients to be their own provider of health care, shifting epilepsy management from professionals to self-care by patients equipped with appropriate skills and tools. 


\section{Background}

Epilepsy is a chronic disorder of the brain, characterized by recurrent seizures. Seizures are the result of sudden, excessive electrical discharges in a group of brain cells. Different parts of the brain can be the site of such discharges, resulting in a variety of clinical manifestations.'

Epilepsy has a considerable psychological and emotional impact, which is strongly reflected in a reduced quality of life (QOL) for patients suffering from epilepsy. ${ }^{2,3}$ Living with seizures is likely to affect patients' daily activities, as it interferes with many aspects of everyday life. Furthermore, epilepsy has been shown to have a large economic impact on society as a whole. ${ }^{4,5}$ For example, the unemployment rate among epileptic patients is at least twice as high as in the general population. ${ }^{6-8}$

Recent studies have shown that up to $70-80 \%$ of newly diagnosed epilepsy patients can be treated successfully (i.e. seizures completely controlled) with anti-epileptic drugs $(A E D s)^{9-11}$ and it is estimated that currently more than 80,000 patients in the Netherlands are treated with AEDs. ${ }^{12}$ However, to achieve and maintain successful seizure control, adherence to treatment is of major importance. A systematic review argued that effective ways of helping people follow medical treatments could have far larger effects on health than any treatment itself. ${ }^{13}$ In addition, it has been recommended that the cost-effectiveness of adherence interventions should be a research priority in the field of chronic diseases. ${ }^{14}$ In epilepsy, poor adherence has been shown to be the most important cause of poorly controlled epilepsy. ${ }^{15}$ However, this study is striving to improve 'concordance' and not only 'adherence'. The crucial difference is that 'adherence' describes only the extent to which a patient takes antiepileptic drugs as prescribed with respect to dosage and dosing intervals, ${ }^{16}$ while 'concordance' includes a consensual agreement about taking AEDs that has been established between patient and practitioner. ${ }^{17}$

Concordance with medical treatment is closely linked with the patients' ability to selfmanage their disease, and the latter is shown to be an important factor in determining quality of life. ${ }^{18}$ Self-management programs focus on supporting patients in coping with their chronic condition, eventually to maximize quality of life, ${ }^{19}$ and have been identified as useful for individuals with chronic conditions such as asthma, heart disease, diabetes. ${ }^{19,20}$ However, due to several reasons, results of studies relating to chronic patient groups cannot be generalized to patients with epilepsy. For example, the consequences of poor self-management, i.e. not taking AEDs or irregular sleeping patterns, are not always directly observable; seizure deregulation can appear the same day or a couple of days later. Hence, the direct link between poor disease management and the frequency of seizures is not transparent for epilepsy patients. A recent study showed that many patients with epilepsy seem to be unaware of missed doses, indicating the need for pill dispensers and reminding/educational interventions. ${ }^{21}$

Self-efficacy, defined as the confidence to carry out a behavior necessary to reach a desired goal ${ }^{19}$ an important concept in self-management. Self-efficacy and changes in selfefficacy are associated with future health status and it appears that enhanced self-efficacy 
is at least one of the mechanisms responsible for the improvements in health status demonstrated by those attending self-management programs. ${ }^{22-24}$ Working within the field of epilepsy, Pramuka et al. ${ }^{20}$ piloted a psychosocial self-management program for epilepsy and observed a positive correlation between self-efficacy and quality of life. In addition, Amir et al. ${ }^{18}$ emphasized the possibility of increasing quality of life among patients with epilepsy by counseling and treatment aimed at increasing their self-efficacy. However, the Managing Epilepsy Well network recently concluded that too few selfmanagement programs exist in general and that there are too few evidence-based programs available. ${ }^{25}$

This study will therefore evaluate the feasibility and (cost-)effectiveness of a multicomponent intervention $(\mathrm{MCl})$, which combines a self-management/education program with e-Health interventions, aiming to improve self-efficacy and concordance in people with epilepsy, in comparison with care as usual (CAU). Hence the $\mathrm{MCl}$ focuses on increasing patients' understanding of their medical regimens, and on providing skills and tools to strengthen self-management and communication between patient and healthcare professional and increase adherence (as a proxy for concordance).

\section{Methods}

This study will consist of three parts, each with its own research questions:

\section{Clinical effectiveness}

1) Is $\mathrm{MCl}$, in comparison with $\mathrm{CAU}$, more effective in terms of self-efficacy and other patient-reported outcomes (self-efficacy, adherence, decrease in seizure frequency \& severity, side effects of AED, controlling depression/anxiety, proactive coping, improved quality of life, and societal costs)?

2) Does the $\mathrm{MCl}$ have a clinical superiority over $\mathrm{CAU}$ in terms of a better adherence to AEDs?

\section{Economic evaluation}

1) What is the cost-effectiveness and the cost-utility of the $\mathrm{MCl}$ in comparison with CAU from a societal perspective?

\section{Process evaluation}

1) Has the $\mathrm{MCl}$ been delivered according to protocol? And if not, what are the reasons for protocol deviation?

2) What are the experiences and opinions of patients, caregivers and professionals regarding the $\mathrm{MCl}$ ? 
3) To what extent has the $\mathrm{MCl}$ impacted concordance among patients (i.e. do patients understand why it is relevant to take the AEDs, and has the $\mathrm{MCl}$ impacted shared decision making by means of consensual agreement between patient and doctor regarding the medical regimen?)

\section{Design}

A randomized controlled trial (RCT) with two parallel groups will be conducted to compare the $\mathrm{MCl}$ with a control situation in epilepsy patients. Patients assigned to the intervention group will attend the $\mathrm{MCl}$ consisting of five weekly sessions and one booster session. The control group will receive CAU as naturally as possible. The follow-up of patients in the intervention group will be 12 months and the follow-up of patients assigned to the control group will be 6 months, after which patients in the control group have the opportunity to receive the $\mathrm{MCl}$ outside the study (see Figure 9.1). The study has been approved by the Ethics Committee of University Hospital Maastricht, the Netherlands.

\section{Study population}

One hundred eligible epilepsy patients will be included in the study. Eligible patients are adults aged 18 or over, living at home, diagnosed with epilepsy and using AEDs, who understand the Dutch language, and are willing and able to use e-Health devices belonging to the $\mathrm{MCl}$. Patients will be excluded if they are not able or willing to function in group activities or when it is expected, on the basis of clinical judgment, that patients are not able to comprehend topics discussed within the $\mathrm{MCl}$ (i.e. patients with cognitive deficits).

\section{Setting \& recruitment}

This study is a collaboration between Maastricht University and the epilepsy center Kempenhaeghe $(\mathrm{KH})$. The study will be conducted at the outpatient clinics of $\mathrm{KH}$. The first $\mathrm{MCl}$ will be offered at the outpatient clinic at the main location of $\mathrm{KH}$ (Heeze), after which the $\mathrm{MCl}$ will be offered at other outpatient clinics (i.e. Nijmegen and Maastricht). From April 2014 onwards, neurologists and nurse practitioners (NP) will recruit possible candidates for participation during consultations at the outpatient clinics of $\mathrm{KH}$. Furthermore, a press release will be published in national epilepsy magazines and on social media, and patient information leaflets will be distributed to patients in the outpatient clinics of $\mathrm{KH}$. In the press release patients are invited to send their contact information to the researchers. 


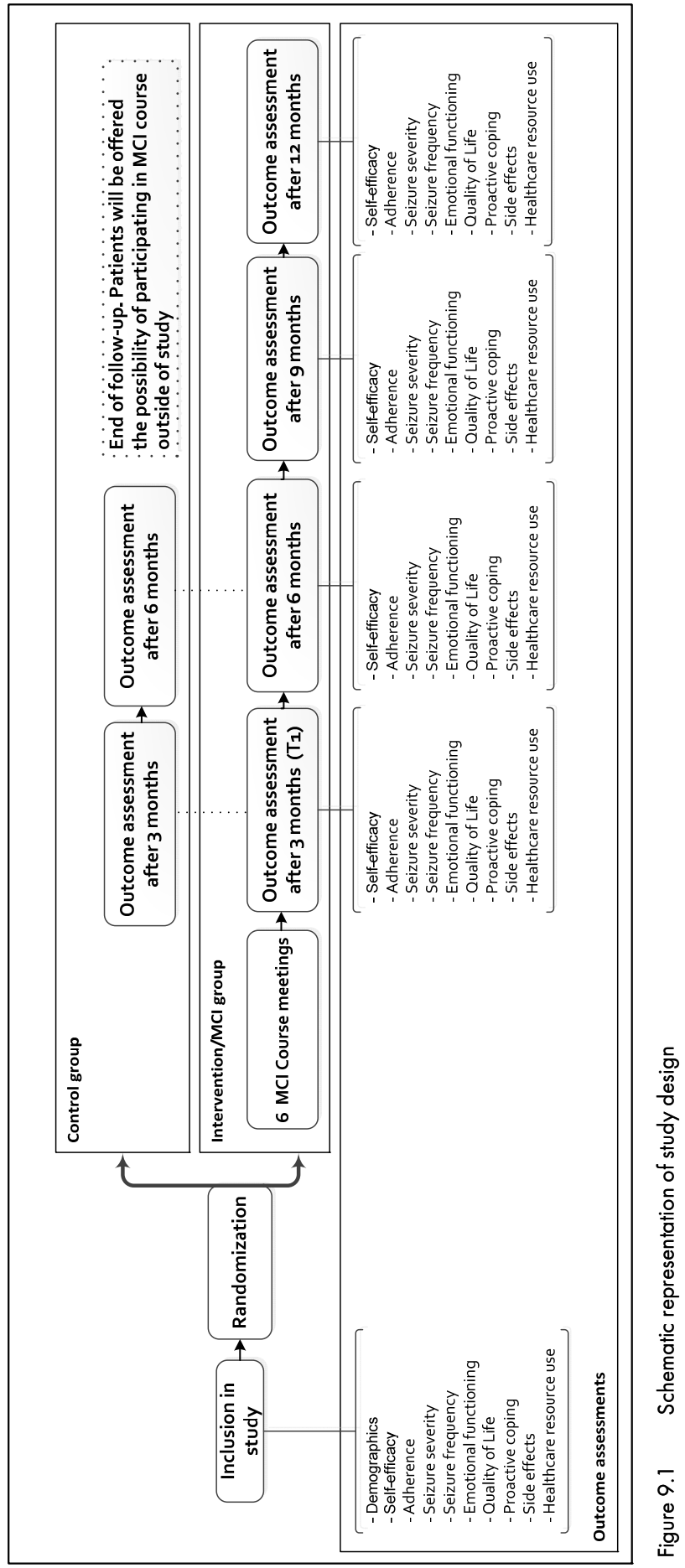


When patients show interest in participating, an information meeting will be scheduled with one of the researchers (LAML or BFMW), either by phone or face-to-face, in which patients will have the opportunity to ask questions and in which inclusion and exclusion criteria will be checked either by the researchers during the meeting or afterwards in consultation with clinicians. During the meeting, patients are informed about the study and that they will be randomly assigned either to the intervention or control group. After one week researchers will contact patients who have received information and want to take part in the study to plan a visit. During this visit an informed consent form will be signed by the patient and the researcher, and patients will be allocated randomly to either the intervention or control group.

\section{Sample size}

The primary outcome variable of this study will be self-efficacy as measured on the Epilepsy Self-Efficacy Scale 33 (ESES). ${ }^{26}$ In a previous pilot study exploring the effects of a psychosocial self-management program for epilepsy patients, with self-efficacy as the primary outcome, the difference between groups was approximately 10 points, with a standard deviation of 7 points on the ESES. ${ }^{20}$ Assuming that alpha $=0.05$ and power $p=0.90$, a minimally detectable difference of 5 points between the intervention and control groups, we will need to include 42 patients per group. Based on a dropout rate of $20 \%$ we intend to include 50 patients in each group.

\section{Randomization}

Patients will be randomized to the $\mathrm{MCl}$ (intervention group) or to the control group, in which patients will be given the opportunity to attend the $\mathrm{MCl}$ outside of the study after six months of follow-up. For parallel provision of both groups (intervention \& control), two blocks of five patients are needed at the moment of randomization. Patients will be assigned to the intervention group or the control group by means of block randomization, using blocks of 10 patients. An assistant who is not involved in the treatment nor in the trial will execute the procedure with a randomization program (www.randomization.com).

\section{Multi-component intervention (MCI)}

Self-management is a process in which patients take responsibility for changing their health behavior by acquiring knowledge about their disease and treatment, and by managing symptoms as well as the physical and psychosocial consequences of the disease. ${ }^{27}$

This definition is a compilation of a broad range of definitions about self-management, combining physical functioning and outcomes with knowledge and the psychosocial consequences of disease. Our $\mathrm{MCl}$ is based on this idea. In order to manage symptoms or the physical and psychosocial consequences, we will try to provide patients with knowledge about self-monitoring (by use of e-health tools) and risk management. In 
addition, in order to change behavior, emphasis will be put on proactive coping, concordance and goal-setting.

Patients will have the opportunity to bring one of their relatives/friends for social support, based on the fact that self-management is not only supported by healthcare providers, but also by the people surrounding persons with chronic diseases. ${ }^{27}$ The intervention is based on the self-management intervention offered in the Restore4Stroke study ${ }^{28}$ but was adjusted to make it suitable for epilepsy patients. The final version of the intervention was developed in agreement with experts in the field of self-management, coordinators of the Restore4Stroke study, clinicians, and representatives of the Epilepsy Association of the Netherlands (EVN, Epilepsie Vereniging Nederland).

The intervention will be explained in a detailed protocol for the NP offering the intervention in the outpatient clinic and in a workbook for patients and one of their relatives/friends. In addition, NPs will receive training beforehand on motivational interviewing (MI), as a technique to empower patients to set their own sustainable goals and look into conflicting beliefs. $\mathrm{Ml}$ is defined as "a collaborative, person-centered form of guiding to elicit and strengthen motivation for change". ${ }^{29} \mathrm{MI}$ focuses on exploring and resolving ambivalence and centers on motivational processes within the individual that facilitate change. ${ }^{30}$

\section{Group sessions}

The $\mathrm{MCl}$ is offered as a group treatment to groups of 5 patients and additional family members and/or friends. The $\mathrm{MCl}$ will last 9 weeks. During the first 5 weeks, group sessions will take place once a week, followed by a booster session 3 weeks later. The group sessions last 2-2.5 hours and are led by an NP with experience in working with epilepsy patient groups. The first session is aimed at providing information about the $\mathrm{MCl}$, including materials, and getting to know the other participants and therapists. During the next sessions, participants will practice with the five stages of proactive coping defined by Aspinwall \& Taylor. $^{31}$ These five stages are (1) resource accumulation, (2) recognition of potential stressors, (3) initial appraisal, (4) preliminary coping efforts, and (5) elicitation and use of feedback concerning initial efforts.

The model will be applied by the patients to three fixed epilepsy-related themes. The first theme will be self-monitoring and self-monitoring (e-Health) tools. The other two themes will be risk-management and shared decision-making/concordance.

Each group session will have the same basic structure. The sessions will begin by looking back at goals set in the previous meeting and how things have worked in the last week. Next, the theme of the session will be introduced. Patients and caregivers will be invited to share their beliefs, emotions and experiences with regard to the theme. Subsequently, patients and caregivers will formulate their own action plan in order to attain a goal relevant to the theme. Patients will be instructed to keep their goals feasible, and group members will give feedback on the quality of the goals in terms of concreteness and attainability. They will help each other to recognize additional conditions and barriers 
which need to be addressed. After the feedback discussion, patients and caregivers will formulate their final plan.

\section{e-Health}

The e-Health tools used in the intervention consist of 3 elements: 1) the Medication Event

Monitoring System (MEMS; Aardex Ltd., Switzerland), 2) a smartphone application, and 3) an internet accessible patient database. The MEMS caps are electronic caps that fit on standard pill bottles. They register the date and time every time the pill bottle is opened. In addition, the MEMS of the intervention group will include an LCD-screen which provides feedback on the number of times the bottle is opened on a particular day. These data can be downloaded from the MEMS cap by the researchers with a communication device and a computer. A computer program will then present the data in simple plots which can be used to provide feedback about behavior, and to identify sub-optimal adherence patterns. $^{32}$ Feedback will be provided during the $\mathrm{MCl}$ and during each follow-up visit. The smartphone application ("Eppy"; Epilepsy Foundation, the Netherlands) is designed to register seizure frequency and other facts for persons with epilepsy, in order to provide data which can influence the management of epilepsy. "Eppy" can be downloaded at no charge from the App store (Apple Inc., USA) or from the Google Play store (Google Inc., USA). The application provides patients with the opportunity, among others, to keep a seizure diary, to set reminders for the intake of medicines and an alarm mode in which a text will be shown for bystanders in case of a seizure. Data gathered with "Eppy" can be synchronized to an internet accessible database, which gives an overview of all clinical events registered by the patient. This internet website is owned by the patient, who can allow healthcare professionals to access it for clinical and research purposes.

\section{Booster session}

During the booster session the NP will rehearse goal setting themes and other themes discussed in the group sessions. Patients and caregivers will have the opportunity to discuss their experiences related to their goals and other aspects of the intervention.

\section{Control group}

The control condition will be a control condition with unrestricted access to CAU. Care will not be intensified after enrollment. As this will be a pragmatic trial, CAU will not follow a standardized protocol. However, medical support will be documented in the electronic patient file of each patient. Medical support provided in the control group might be variable, but is expected to be in agreement with the standard epilepsy guidelines (i.e. preference for mono-drug therapy, a monitoring visit every 4 months by neurologist or epilepsy nurse and change or addition of medication if the first AED is ineffective). ${ }^{33}$ The control group will receive MEMS but without feedback about their behavior. The MEMS 
will be read only at the end of the follow-up as a way to measure adherence and will not include an LCD-screen.

\section{Clinical effectiveness}

To assess the (cost)-effectiveness of the $\mathrm{MCl}$, outcome assessments will be done at baseline and after 3, 6, 9, and 12 months $(9$ and 12 months applicable only for intervention group). A detailed overview regarding the outcome assessments can be found in Table 9.1. The following outcomes are defined:

\section{Primary outcomes}

1) Epilepsy Self-Efficacy Scale-33 items (ESES). The ESES is a 33-item scale that measures different aspects of efficacy within the self-management of epilepsy. The items represent three dimensions of self-management: medication management, seizure management, and general management including safety and health. ${ }^{26}$ Items are rated on an 11-point Likert rating scale, ranging from 0 , "not at all certain I can do", to 10, "very certain I can do". ${ }^{34}$ The total possible scores for the ESES range from 0 to 330 . Higher scores correspond to higher levels of confidence in the ability to manage epilepsy.

\section{Secondary outcomes}

1) Adherence, used as a proxy for concordance, which will be determined using: 1) MEMS. Electronic monitoring, such as MEMS has been proposed as a possible "gold standard" for medication adherence measurement. ${ }^{15,35}$ and 2) the Medication Adherence Rating Scale (MARS-5), which measures self-reported adherence. The MARS-5 contains 10 items, each of which has to be answered with yes or no. Hence, the final score on the MARS-5 ranges from 0 to 10 , in which a higher score represents better adherence.

2) General self-efficacy, which will be determined using the Dutch adaptation of the general Self-Efficacy Scale (GSES). The GSES consists of 10 items assessed on a 4-point scale, ranging from 'totally wrong' to 'totally true'. The scale was designed to assess self-efficacy, i.e., the belief that one's actions are responsible for successful outcomes. The scale scores for each question ranges from 1 to 4 resulting in an overall score between 10 and 40. Higher scores indicate patient's stronger belief in self-efficacy. ${ }^{36,37}$

3) Seizure frequency will be determined using a short questionnaire regarding seizure frequency covering the past 4 weeks. The questions focus mainly on the number of seizures, whether the person documents his/her seizures and in what way the person documents his/her seizures.

4) Seizure severity, which will be determined using the National Hospital Seizure Severity Scale (NHS3). The NHS3 lists seven seizure-related factors and 
generates a score from 1 to 27 , in which a higher score represents a more severe seizure. $^{38}$

5) Adverse events of AED, which will be determined using the SIDe-effect of the AntiEpileptic Drugs questionnaire (SIDAED). The SIDAED consists of 46 items regarding possible AED-related complaints. These items form 10 categories: general CNS, behavior (increased irritability), depressive symptoms, cognitive function, motor problems and co-ordination, visual complaints, headache, cosmetic and dermatological complaints, gastrointestinal complaints, and sexuality and menses. ${ }^{39}$ For each item the patient rates the severity of the complaint on a four- point Likert scale (no problem, mild, moderate, or serious problem). In addition, the duration of the complaints is scored (a few weeks, months or half a year or longer). The SIDAED ranges from 0 to 138, in which a higher score indicates more severe/frequent side-effects. ${ }^{39}$

6) Depression/Anxiety, which will be determined using the Hospital Anxiety and Depression Scale (HADS). The HADS has a total of 14 items, each scored on a scale of $0-3$, with 3 indicating higher symptom frequencies. Scores for each subscale (anxiety and depression) can range from 0-21 and scores for the entire scale (emotional distress) range from $0-42$, with higher scores indicating more distress. $^{40}$

7) Self-rated proactive coping, which will be determined using the Utrecht Proactive Coping Competence Scale (UPPC). A total of 21 items are assessed on a 4-point scale ranging from 'not competent at all' to 'very competent'. Total scores are calculated by averaging the 99 individual item scores. Higher scores on the UPCC indicate higher levels of perceived proactive coping competencies. ${ }^{41}$

8) Disease-specific quality of life will be measured with the Quality Of Life in Epilepsy-patient-weighted (QOLIE-31-P). The QOLIE-31-P consists of 38 items assessing 7 domains of epilepsy: Seizure Worry, Overall QOL, Emotional wellbeing, Energy-Fatigue, Cognitive Functioning, Medication Effects, Social Functioning and an Overall Score. In addition, for each domain, questions regarding how much distress a person feels about problems and worries related to epilepsy are included. ${ }^{42}$ Each domain is scored on a scale ranging from 0 to 100. Afterwards a final score can be calculated using weights derived from the amount of distress related to each domain. The final score ranges between 0 to 100, in which higher values indicate a better Quality Of Life. ${ }^{43}$

9) Generic Quality Of Life will be assessed with the EuroQoL 5 dimensions 5 levels (EQ-5D-5L). The EQ-5D-5L consists of five dimensions: mobility, self-care, usual activities, pain/discomfort, anxiety/depression, each of which can have one of five responses. ${ }^{44,45}$ Each health state will be valued using the Dutch tariffs, which will result in utilities on a scale from 0 to 1 . Utilities derived from the EQ-5D-5L will be used in calculating the quality adjusted life years (QALY) by multiplying the time spent in a health state by the utility assigned to that health state. 
10) Societal costs will be measured retrospectively with the a Medical Cost Questionnaire (MCQ), an adapted version of the Trimbos/iMTA questionnaire for costs associated with psychiatric illness ${ }^{46}$ and the Productivity Cost Questionnaire (PCQ), each covering 3 months.

Table 9.1 Overview of measurements per time point.

\begin{tabular}{|c|c|c|c|c|c|c|c|}
\hline Outcomes Patients & Instrument & Short term & TO & $\mathrm{Tl}$ & $\mathrm{T} 2$ & $\mathrm{~T} 3$ ** & $\mathrm{T} 4$ ** \\
\hline $\begin{array}{l}\text { Demographic and clinical } \\
\text { characteristics }\end{array}$ & - & - & $X$ & - & - & - & - \\
\hline Self-efficacy & $\begin{array}{l}\text { Epilepsy Self-efficacy Scale - } 33 \\
\text { items }\end{array}$ & ESES & $x$ & $x$ & $x$ & $\mathrm{x}$ & $x$ \\
\hline General Self-efficacy & $\begin{array}{l}\text { Generic Self-efficacy Scale - } 10 \\
\text { items }\end{array}$ & GSES & $x$ & $x$ & $x$ & $x$ & $x$ \\
\hline Adherence & $\begin{array}{l}\text { MEMS } \\
\text { Medication Adherence Scale }\end{array}$ & MARS 5 & $\mathrm{x}$ & $x$ & $x$ & $\mathrm{x}$ & $x$ \\
\hline Seizure frequency & Questionnaire seizure frequency & - & $\mathrm{X}$ & $x$ & $\mathrm{x}$ & $\mathrm{X}$ & $\mathrm{X}$ \\
\hline Seizure severity & $\begin{array}{l}\text { National Hospital Seizure Severity } \\
\text { Scale }\end{array}$ & NHS3 & $x$ & $x$ & $x$ & $x$ & $x$ \\
\hline Emotional functioning & $\begin{array}{l}\text { Hospital Anxiety and Depression } \\
\text { Scale }\end{array}$ & HADS & $\mathrm{X}$ & $x$ & $x$ & $\mathrm{x}$ & $x$ \\
\hline Quality of life & $\begin{array}{l}\text { Quality Of Life in Epilepsy } \\
\text { Generic quality of life }\end{array}$ & $\begin{array}{l}\text { QOLIE3 1P } \\
\text { EQ-5D-5L }\end{array}$ & $\begin{array}{l}X \\
X \\
\end{array}$ & $\begin{array}{l}X \\
X \\
\end{array}$ & $\begin{array}{l}X \\
X \\
\end{array}$ & $\begin{array}{l}X \\
X \\
\end{array}$ & $\begin{array}{l}X \\
X \\
\end{array}$ \\
\hline Proactive Coping & $\begin{array}{l}\text { Utrecht Proactive Coping } \\
\text { Competence }\end{array}$ & $\mathrm{UPCC} / \mathrm{PCl}$ & $\mathrm{X}$ & $x$ & $x$ & $x$ & $x$ \\
\hline Side Effect & Side effects of Anti-epileptic Drugs & SIDAED & $\mathrm{X}$ & $x$ & $x$ & $x$ & $\mathrm{X}$ \\
\hline Healthcare resource use & $\begin{array}{l}\text { Medical Cost Questionnaire } \\
\text { Productivity Cost Questionnaire }\end{array}$ & $\begin{array}{l}M C Q \\
P C Q\end{array}$ & $x$ & $x$ & $x$ & $x$ & $x$ \\
\hline
\end{tabular}

${ }^{*} \mathrm{TO}=$ baseline outcome assessments; $\mathrm{T} 1=$ Outcome assessments after 3 months; $\mathrm{T} 2=$ Outcome assessments after 6 months; T3=Outcome assessments after 9 months; T4=Outcome assessments after 12 months. * Outcome assessment applicable only for intervention group

\section{Economic evaluation}

The trial-based economic evaluation will be performed from a societal perspective and will consist of a cost-effectiveness analysis (CEA) and a cost-utility analysis (CUA). Outcomes of interest for the CEA and the CUA will be self-efficacy as assessed by the ESES, and generic quality of life as assessed by EQ-5D-5L. We distinguish four cost categories: intervention costs, healthcare sector costs, costs for the patient and family, and productivity costs. Intervention costs will be defined as all costs related to the $\mathrm{MCl}$ including travel costs, personal costs, material costs, costs of e-Health tools (i.e. MEMS) and housing costs. Healthcare and patient costs will be estimated using a questionnaire regarding healthcare resource utilization and productivity losses.

Resource use and outcomes are measured at the same time points mentioned in the effectiveness study: at baseline, 3 months, 6 months, 9 months, and 12 months in the intervention group and at 3 months and 6 months in the control group. A comparison between $\mathrm{MCl}$ and $\mathrm{CAU}$ will be made in terms of incremental costs and incremental effects. 
The time horizon will be 12 months (trial-based economic evaluation). To measure the use of health care resources, including all activities related to epilepsy, we gather data for each patient at baseline and at a follow-up of one year. Cost calculations will be based on the Dutch guidelines for cost calculations in healthcare. ${ }^{47}$

\section{Process evaluation}

Process evaluation will be performed to assess whether the $\mathrm{MCl}$ was delivered according to protocol, to examine the experiences and opinions of patients, caregivers and professionals regarding the $\mathrm{MCl}$, and to determine to what extent the $\mathrm{MCl}$ has impacted concordance among patients. The process evaluation will be performed according to the framework provided by Saunders et al.. ${ }^{48}$ This framework consists of a stepwise approach in which important characteristics for the process-evaluation plan are identified along seven basic components, namely: fidelity (quality), dose delivered (completeness), dose received (exposure), dose received (satisfaction), reach (participation rate), recruitment and context.

We will use a mixed methods design in which both qualitative and quantitative data will be collected. The qualitative part will consist of observations during several group sessions over time. After each observation, a short interview will be held with the group leader(s) in which the group leader can reflect on his/her opinion regarding the session. During the last (sixth) group session of every $\mathrm{MCl}$-group, a short evaluation form will be handed out to patients in which they will be asked to rate different aspects and themes of the $\mathrm{MCl}$ on a 7-point Likert scale. Furthermore, at the end of the study, focus groups will be held consisting of patients included in the study. The selection of participants will be based on maximal variation to get as many perspectives as possible. Participants will be selected based on age, sex and severity of seizures and effectiveness of the intervention (to compare patients for which the intervention was successful versus unsuccessful). Focus groups will be conducted using a semi-structured questionnaire covering the topics identified in the framework provided by Saunders et al.. ${ }^{48}$

\section{Analysis}

\section{Clinical effectiveness}

Baseline characteristics will be described, and differences between groups at baseline will be studied using t-tests or chi-square tests where appropriate. All statistical procedures will be conducted based on both the intention-to-treat principle and on actual participation in treatment (i.e. effectiveness analyses) and will be performed using SPSS statistics 22.0 (SPSS, IBM, Corporation, Chicago, USA). Missing data will be handled using SPSS missing value analysis on item level. Completely missing measurements will be handled using multiple imputation. To evaluate outcomes, change scores will be calculated and compared between the groups after treatment. Multi-level analyses will be performed with measurements (TO, T1, T2, T3 and T4) within the subjects' factor and group and between 
subjects' factors to account for the nested structure of the data. Data from the control group will be extrapolated to 12 months. Post hoc analyses will be performed in case of significant effects. Baseline differences will be corrected by inclusion of covariates in the analyses. A 2-sided significance level of 0.05 will be used as a threshold to determine whether differences are statistically significant.

\section{Economic evaluation}

Costs calculation will be performed according to the bottom-up approach, based on a detailed inventory of all cost items. Standardized cost prices from the Dutch manual for costing will be used in the calculations or (if not available) calculated mean cost prices, according to providers, will be used. ${ }^{47}$ To determine the costs of drugs, the Dutch consumer reimbursement price for medication will be used. Productivity costs of the patients will be estimated with the friction cost method. Costs will be indexed for the year 2015.

As cost data are normally skewed, parametric tests are mostly not suitable. Hence nonparametric bootstrapping (1000 times) will be used to test for statistical differences in costs between groups and to investigate the uncertainty around the costs. Bootstrapping will be done using Microsoft Excel (Excel, Microsoft Corporation, Washington, USA). Bootstrap replications will be used to calculate $95 \%$ confidence intervals around the costs, based on the 2.5th and 97.5th percentiles. For the cost-effectiveness analysis, the corresponding incremental cost-effectiveness ratio (ICER) will be expressed as incremental costs per increased adherence and incremental costs per self-efficacy (ESES). In the cost-utility analysis, the ICER will be expressed as the incremental costs per QALY gained. QALYs will be calculated using the area under the curve method. All bootstrapped ICERs (5000 times) will be presented in a cost-effectiveness plane to determine the robustness of the ICER. To determine the probability that the $\mathrm{MCl}$ is cost-effective given a certain ceiling ratio, a costeffectiveness acceptability curve will be constructed. In addition, one-way and multi-way sensitivity analysis will be performed on the most important cost parameters.

\section{Process evaluation}

Quantitative data will be analyzed by descriptive statistics (i.e. frequencies, mean and median), Chi square tests, and ANOVA. Results from open-ended questions included in the questionnaires, focus group interviews and interviews will be categorized to identify relevant themes.

\section{Discussion}

This study will determine the (cost-)effectiveness and feasibility of the $\mathrm{MCl}$ to improve the management of epilepsy in adult patients and increase self-efficacy and concordance regarding AED. The $\mathrm{MCl}$ is designed to stimulate self-management skills and the awareness of patients with epilepsy in combination with the use of e-Health interventions. 
In times of budget constraints, $\mathrm{MCl}$ could be a valuable addition to current healthcare provisions for epilepsy, as it is expected that higher concordance and self-efficacy will result in reduced healthcare resource use and an increased QOL.

Cooperation between research and practice is the key strength of this project, enabling the intervention to be studied in a natural environment; the project will facilitate further implementation of the multi-component program into the standard practice of $\mathrm{KH}$ and of other institutions. Both professionals and patients played important roles in the development of this program and will also be involved during the evaluation and implementation of the $\mathrm{MCl}$ program.

One of the limitations of our study design is that, in case the $\mathrm{MCl}$ is shown to be effective, it will be difficult to identify what components contribute to this effectiveness. For example, it could be that only the increased attention from nurses or the adherence monitoring contribute to improvements in the patients' health status. However, we believe we have included a large variety of outcome assessments which, altogether, form a broad view of the (possible) effectiveness of $\mathrm{MCl}$.

To the best of our knowledge, there are currently no validated questionnaires available to examine concordance. However, as this study is striving to improve 'concordance' and not only 'adherence', it is assumed that any increase in adherence by the $\mathrm{MCl}$ is partly explained by increased concordance (i.e. due to the educational content in the $\mathrm{MCI}$ ). In addition, special attention will be paid to concordance during the process evaluation. Accordingly, this study is aimed at making patients their own provider of health care, thus shifting epilepsy management from professionals to self-care by patients equipped with appropriate skills and tools. 


\section{References}

1. Fact sheet Epilepsy [http://www.who.int/mediacentre/factsheets/fs $999 /$ en/index.html]

2. Jacoby A, Baker GA: Quality-of-life trajectories in epilepsy: a review of the literature. Epilepsy Behav 2008; 12(4):557-71.

3. Taylor RS, Sander JW, Taylor RJ, Baker GA: Predictors of health-related quality of life and costs in adults with epilepsy: a systematic review. Epilepsia 2011 1;52(12):2168-80.

4. Kotsopoulos IA, Evers SM, Ament AJ, de Krom MC: Estimating the costs of epilepsy: an international comparison of epilepsy cost studies. Epilepsia 2001;42(5):634-40.

5. Kotsopoulos IA, Evers SM, Ament AJ, Kessels FG, de Krom MC, Twellaar M, Metsemakers JF, Knottnerus AJ: The costs of epilepsy in three different populations of patients with epilepsy. Epilepsy Res 2003;54(23): 131-40.

6. Elwes R, Marshall J, Beattie A, Newman P: Epilepsy and employment. A community based survey in an area of high unemployment. J Neurol Neurosurg Psychiatry 1991;54(3):200-3.

7. Hart YM, Shorvon SD: The nature of epilepsy in the general population. I. Characteristics of patients receiving medication for epilepsy. Epilepsy Res 1995;21(1):43-9.

8. Pato Pato A, Cebrian Perez E, Cimas Hernando I, Lorenzo Gonzalez JR, Rodriguez Constenla I, Gude Sampedro F: Analysis of direct, indirect, and intangible costs of epilepsy. Neurologia (Barcelona, Spain) $2011 ; 26(1): 32-8$.

9. Strzelczyk A, Reese J, Dodel R, Hamer H: Cost of Epilepsy. Pharmacoeconomics 2008, 26(6):463-476.

10. Loiseau J, Picot M-C, Loiseau P: Prognosis of epileptic syndromes: EPIGIR study. Prognosis of Epilepsies Montrouge: John Libbey 2003:29-38.

11. Kwan P, Brodie MJ: Early identification of refractory epilepsy. N Engl J Med 2000;342(5):314-9.

12. Wallace $H$, Shorvon $S$, Tallis R: Age-specific incidence and prevalence rates of treated epilepsy in an unselected population of 2052922 and age-specific fertility rates of women with epilepsy. Lancet, 1998;352(9145):1970-3.

13. Haynes RB, Ackloo E, Sahota N, McDonald HP, Yao X: Interventions for enhancing medication adherence. The Cochrane database of systematic reviews 2008(2):CD0000 11.

14. Oberié EJ, de Kinderen RJ, Evers SM, van Woerkum CM, de Bruin M: Cost Effectiveness of Medication Adherence-Enhancing Interventions: A Systematic Review of Trial-Based Economic Evaluations. Pharmacoeconomics 2013;31(12):1155-68.

15. Jones RM, Butler JA, Thomas VA, Peveler RC, Prevett M: Adherence to treatment in patients with epilepsy: associations with seizure control and illness beliefs. Seizure 2006;15(7):504-8.

16. Cramer JA, Roy A, Burrell A, Fairchild CJ, Fuldeore MJ, Ollendorf DA, Wong PK: Medication compliance and persistence: terminology and definitions. Value Health 2008;1 1(1):44-7.

17. Haynes RB, Ackloo E, Sahota N, McDonald HP, Yao X: Interventions for enhancing medication adherence. Cochrane database syst Rev 2008, 2(2).

18. Amir M, Roziner I, Knoll A, Neufeld MY: Self-Efficacy and Social Support as Mediators in the Relation Between Disease Severity and Quality of Life in Patients with Epilepsy. Epilepsia 1999;40(2):216-24.

19. Bodenheimer T, Lorig K, Holman H, Grumbach K: PAtient self-management of chronic disease in primary care. JAMA 2002;288(19):2469-75.

20. Pramuka M, Hendrickson R, Zinski A, Van Cott AC: A psychosocial self-management program for epilepsy: A randomized pilot study in adults. Epilepsy Behav 2007;1 1 (4):533-45.

21. Samsonsen C, Reimers A, Brathen G, Helde G, Brodtkorb E: Nonadherence to treatment causing acute hospitalizations in people with epilepsy: An observational, prospective study. Epilepsia 2014; 55(11):e 125-8.

22. Lorig K, Chastain RL, Ung E, Shoor S, Holman HR: Development and evaluation of a scale to measure perceived self-efficacy in people with arthritis. Arthritis \& Rheumatism 1989;32(1):37-44.

23. Lorig K, Gonzalez VM, Ritter P: Community-based Spanish language arthritis education program: a randomized trial. Med Care 1999;37(9):957-63.

24. Lorig KR, Holman HR: Self-management education: history, definition, outcomes, and mechanisms. Ann Behav Med 2003;26(1):1-7.

25. Shegog R, Bamps YA, Patel A, Kakacek J, Escoffery C, Johnson EK, llozumba UO: Managing Epilepsy Well: Emerging e-Tools for epilepsy self-management. Epilepsy Behav 2013;29(1):133-40. 
26. Dilorio C, Yeager K: The epilepsy self-efficacy scale. Teoksessa: Strickland OL \& Dilorio C (toim) Measurement of nursing outcomes: Self care and coping, New York 2003:40-51.

27. Ouwens $M$, van der Brug S, Faber M, van der Weijden T: Shared Decision Making \& Zelfmanagement. In. Nijmegen: Scientific Institute for Quality of Healthcare; 2012.

28. van Eeden $M$, van Heugten $C$, Evers $S$ : The economic impact of stroke in The Netherlands: the Erestore4stroke study. BMC Public Health 2012;12(1):122.

29. Miller WR, Rollnick S: Ten things that motivational interviewing is not. Behav Cogn Psychother 2009;37(2): 129.

30. Miller WR, Rollnick S: Motivational interviewing: Preparing people for change: Guilford press; 2002.

31. Aspinwall LG, Taylor SE: A stitch in time: self-regulation and proactive coping. Psychological bulletin 1997; $121(3): 417$.

32. Data collection MWV Aardex [http://www.mwvaardex.com/Products/DataCollection/index.htm]

33. Neurologie; NVv, Kwaliteitsbevordering; C: Richtlijnen diagnostiek en behandeling van Epilepsie. Herziene, tweede versie: januari 20062006.

34. Dilorio C, Faherty B, Manteuffel B: The development and testing of an instrument to measure self-efficacy in individuals with epilepsy. J Neurosci Nurs 1992;24(1):9-13.

35. Bertholet N, Favrat B, Fallab-Stubi CL, Brunner HR, Burnier M: Why Objective Monitoring of Compliance is Important in the Management of Hypertension. J Clin Hypertens 2000;2(4):258-62.

36. Sherer M, Maddux JE, Mercandante B, Prentice-Dunn S, Jacobs B, Rogers RW: The self-efficacy scale: Construction and validation. Psychological reports 1982;51(2):663-71.

37. Teeuw B, Schwarzer R, Jerusalem M: Dutch adaptation of the general self-efficacy scale. Berlin, Germany 1994.

38. O'Donoghue MF, Duncan JS, Sander JWAS: The National Hospital Seizure Severity Scale: A Further Development of the Chalfont Seizure Severity Scale. Epilepsia 1996;37(6):563-71.

39. Uij SG, Uiterwaal CSMP, Aldenkamp AP, Carpay JA, Doelman JC, Keizer K, Vecht CJ, de Krom MC, van Donselaar CA: A cross-sectional study of subjective complaints in patients with epilepsy who seem to be well-controlled with anti-epileptic drugs. Seizure 2006; 15(4):242-8.

40. Zigmond AS, Snaith RP: The hospital anxiety and depression scale. Acta Psychiatr Scand 1983; $67(6): 361-70$.

41. Tielemans NS, Visser-Meily JMA, Schepers VPM, Post MWM, van Heugten CM: Proactive coping poststroke: Psychometric properties of the Utrecht Proactive Coping Competence scale. Archives of Physical Medicine and Rehabilitation (0).

42. Devinsky O, Vickrey BG, Cramer J, Perrine K, Hermann B, Meador K, Hays RD: Development of the Quality of Life in Epilepsy Inventory. Epilepsia 1995;36(1 1):1089-104.

43. QOLIE-31-PO: Cramer JA, Van Hammee, G., N132 Study Group.: Maintenance of improvement in health-related quality of life during long-term treatment with levetiracetam. Epilepsy \& Behavior 2003, 4:118-23.

44. Brooks R: EuroQol: the current state of play. Health Policy 1996;37(1):53-72.

45. Dolan P: Modeling valuations for EuroQol health states. Med Care 1997;35(11):1095-108.

46. Hakkaart-van Roijen L, Straten Av, Tiemens B, Donker M: Handleiding Trimbos/iMTA questionnaire for Costs associated with Psychiatric illness (TiC-P). In.: Institute of Medical Technology Assessment (iMTA); 2002.

47. Hakkaart-van Roijen L, Tan S, Bouwmans C: Handleiding voor kostenonderzoek. Methoden en standaard kostpriizen voor economische evaluaties in de gezondheidszorg Geactualiseerde versie 2010.

48. Saunders RP, Evans MH, Joshi P: Developing a process-evaluation plan for assessing health promotion program implementation: a how-to guide. Health Promot Pract 2005;6(2):134-47. 



\section{An economic evaluation of}

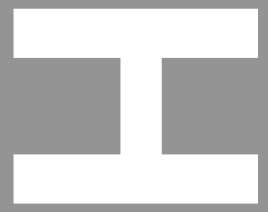

a multi-component self-

management intervention for

adults with epilepsy

(ZMILE study)
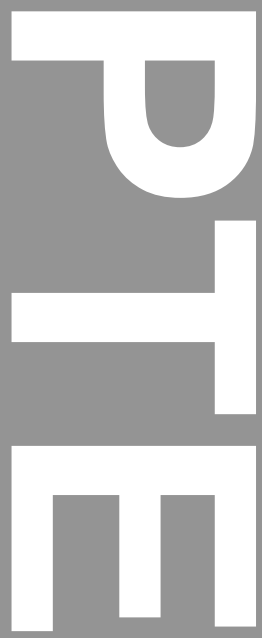

B.F.M. Wiinen

L.A.M. Leenen

R.J.A. de Kinderen

C.M. van Heugten

M.H.J.M. Majoie

S.M.A.A. Evers

Accepted for publication in Epilepsia (in press) 


\section{Abstract}

\section{Objective}

The objective of this (trial-based) economic evaluation was, from a societal perspective, to compare the cost-effectiveness of a multi component self-management intervention ( $\mathrm{MCl}$ ) with care as usual (CAU) in adult patients with epilepsy over a 12 month period.

\section{Methods}

Participants were randomized into intervention or CAU group. Adherence, self-efficacy (ESES), quality adjusted life years (QALYs), health care costs, production losses, and patient \& family costs were assessed at baseline and during the 12-month study period. Incremental costeffectiveness ratios (ICERs) (i.e. cost per increased adherence, self-efficacy or QALY), and cost-effectiveness acceptability curves were calculated and presented.

\section{Results}

In total, 102 patients were included in the study, of whom 52 were in the intervention group. Adherence rates over 6 months were $63.7 \%$ for the CAU group and $75.9 \%$ for the intervention group. Adherence, ESES and quality of life did not differ significantly between groups. An ICER of $€ 54$ per point increase in ESES-score at 6 months and $€ 1105$ per point increase at 12 months follow-up was found. The intervention resulted in an ICER of $€ 88$ per percentage of adherence increase at 6 months. ICERs of $€ 8,272$ and $€ 15,144$ per QALY gained were found at 6 and 12 months followup, respectively.

\section{Significance}

Although no statistically significant difference was found after baseline adjustments, costeffectiveness estimates for $\mathrm{MCl}$ appear to be promising. As rules of inference are arbitrary, it has been argued that decisions should be based only on the net benefits, irrespective of whether differences are statistically significant. Hence, the $\mathrm{MCl}$ may be a cost-effective addition to the current standard care for adults with epilepsy. 


\section{Introduction}

In Europe, the prevalence of epilepsy is estimated to be 5 per 1,000 inhabitants.' As life expectancy is rising, resulting in an aging population, the expectation is that the number of people with a chronic condition such as epilepsy will increase. ${ }^{2}$ In terms of economic impact on society, epilepsy is highly cost-intensive with respect to diagnosis, treatment and hospitalizations due to seizures, as well as unemployment and household work. ${ }^{3,4}$ Furthermore, uncontrolled seizures negatively impact the quality of life (QoL) of patients with epilepsy. ${ }^{5}$ As the healthcare budget is insufficient to cover all healthcare expenses, ${ }^{6}$ economic evaluations are becoming a common requirement for reimbursement decisions. ${ }^{7,8}$ Budget constraints challenge policy makers to make the most cost-effective and health-producing decisions when allocating limited resources.

People with epilepsy are in many ways their own primary carers; well-controlled epilepsy often results in less productivity losses, hospitalizations, and other use of (healthcare) resources. ${ }^{9}$ Higher quality of self-care and reduced health care costs, provision of information and adequate problem-solving skills are needed. Selfmanagement education programs are designed to meet this need. ${ }^{10}$ Evidence from previous controlled clinical trials has indicated positive effects of self-management programs on improving clinical outcomes and their potential to reduce costs for chronic disorders. $^{10,11}$ However, the Managing Epilepsy Well (MEW) Network has revealed the lack of evidence-based programs for epilepsy self-management education. ${ }^{12}$ One of the mechanisms responsible for the improvements in health status, demonstrated by those attending self-management programs, is self-efficacy. ${ }^{10}$ Furthermore, the effectiveness of an AED treatment not only depends on the type of AED; the patients' attitude to it is of great importance. ${ }^{13}$ Hence, adherence, or more precisely concordance, with medical treatment is closely linked to the patients' ability to self-manage their disease, and is shown to be an important factor in determining QoL. ${ }^{14}$ Concordance reflects a consensual agreement about taking AEDs that has been established between patient and practitioner. ${ }^{15}$

In this study, a full economic evaluation was performed examining the costeffectiveness of a multi-component intervention (MCI) aimed at increasing selfmanagement skills for people with epilepsy. Hence, the aim of this study was to compare, from a societal perspective, the cost-effectiveness (i.e. cost per improved adherence or selfefficacy) and cost-utility (i.e. cost per QALY gained) of the $\mathrm{MCI}$ with care as usual (CAU) in adult patients with epilepsy over a period of 6 months with an extended follow-up of 12 months for the $\mathrm{MCl}$ group.

\section{Methods}

\section{Design}

This trial-based economic evaluation was part of a randomized controlled trial (RCT) in which the clinical effectiveness of the $\mathrm{MCl}$ was assessed. Randomization was performed 
using sealed envelopes by an independent person. The study consisted of two parallel groups in which participants were randomly allocated to either the $\mathrm{MCl}$ group la selfmanagement education program with e-Health interventions) or CAU. The control group received CAU. Participants in the intervention group were followed for 12 months and the CAU group for 6 months. After 6 months, patients from the control group were also given the opportunity of receiving $\mathrm{MCl}$; this did not form part of the study. The protocol of this study has previously been described in Leenen \& Wijnen et al. (2014). ${ }^{16}$ An overview of all relevant measurements for this economic evaluation is presented in Figure 10.1.

\section{Population \& Setting}

Adult patients, aged 18 years and over, diagnosed with epilepsy, living at home, using AEDs, who understand the Dutch language, and were willing and able (based on neurologists' opinion) to use e-Health devices belonging to the $\mathrm{MCl}$, were eligible to participate in this study. Patients were excluded if they were not able or willing to function in group activities or when it was expected, on the basis of clinical judgment, that they were not able to comprehend topics discussed within the $\mathrm{MCl}$ (e.g. patients with cognitive deficits).

Patients were recruited in the outpatient clinics of Kempenhaeghe and by means of national epilepsy magazines \& social media. The inclusion started March 2014 and the last follow-up measurements were carried out in November 2016.

\section{Multi-component intervention}

The $\mathrm{MCl}$ consisted of 1) group sessions; 2) the Medication Event Monitoring System (MEMS; Aardex Ltd., Switzerland); 3) a smartphone application "Eppy" (Epilepsy Foundation, the Netherlands); and 4) an internet accessible patient database.

The group sessions consisted of groups of 3-5 patients and additionally family members and/or friends. During the first 5 weeks, group sessions took place once a week, followed by a booster session 4 weeks later. The group sessions lasted for 2 hours and were led by two nurse practitioners, with experience in working in epilepsy patient groups. During the course, participants practiced with the five stages of proactive coping described by Aspinwall \& Taylor ${ }^{17}$ and three fixed epilepsy-related themes. ${ }^{16}$ These five stages were (1) resource accumulation; (2) recognition of potential stressors; (3) initial appraisal; (4) preliminary coping efforts; and (5) elicitation and use of feedback concerning initial efforts. The three epilepsy related themes were: (1) self-monitoring and self-monitoring (e-Health) tools; (2) risk-management; and (3) shared decision-making/concordance. Patients were instructed to set goals and to proactively cope with possible pitfalls. The course was based on a self-management program for patients with type 2 diabetes, which was shown to significantly improve self-efficacy. ${ }^{18}$ 


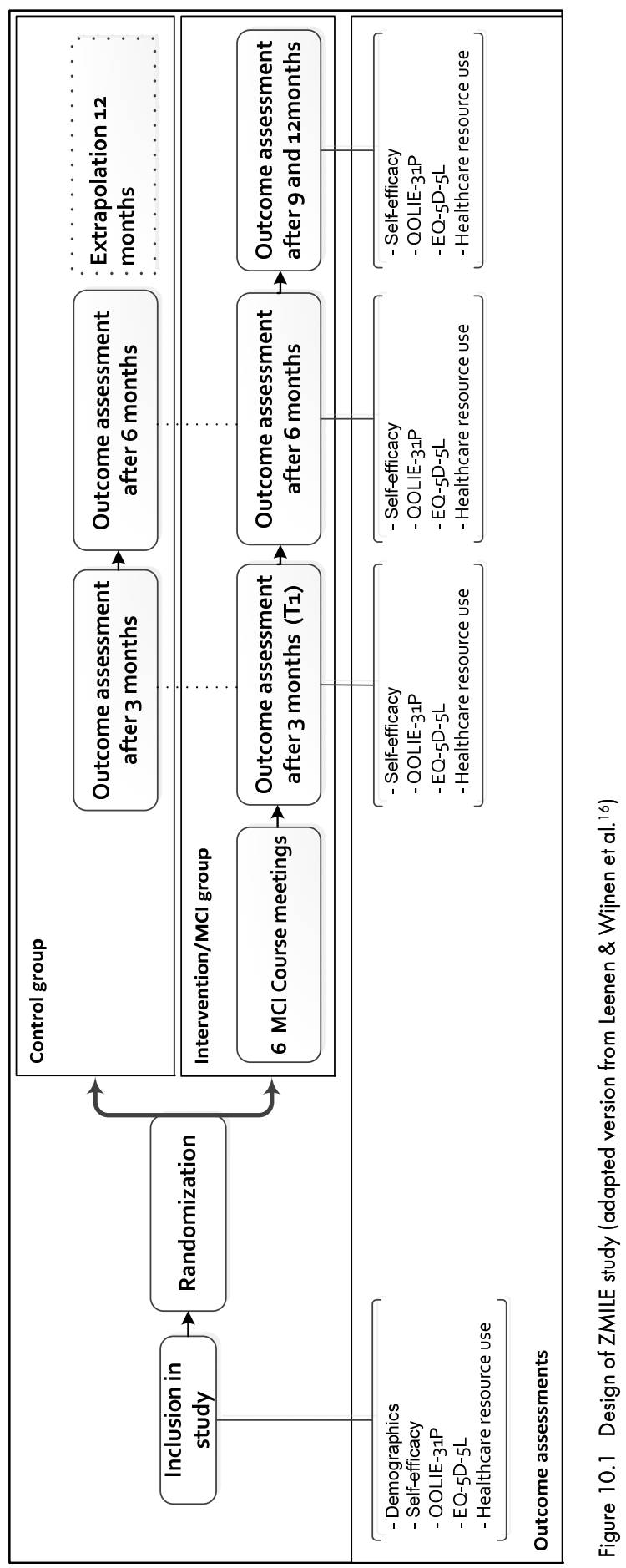


The MEMS caps were electronic caps that fit on standard pill bottles. They registered the date and time every time the pill bottle was opened. In addition, the MEMS of the intervention group included an LCD-screen which provided feedback on the number of times the bottle was opened on a particular day. Patients in the intervention group received feedback on the medication adherence based on MEMS reports during the group sessions and after 3, 6, 9 and 12 months' follow-up. Adherence was expressed as the percentage of days the MEMS bottle was opened according to AED regime, calculated as 100 * (number of days with number of openings as prescribed / number of monitored days).

The smartphone application "Eppy" was designed to register seizure frequency and other facts for persons with epilepsy. The internet accessible patient database consisted of a web-based interface and overview of the information gathered in "Eppy".

\section{Care as usual}

Patients in the CAU group received no intervention; care was not intensified. The CAU group did not follow a standardized protocol. The CAU group also received the MEMS cap but without feedback about their behavior and without a LCD-screen providing feedback regarding the number of times it was opened on a particular day.

\section{Outcomes}

All patients included in the study were administered self-reporting questionnaires at baseline and after 3, 6, 9 and 12 months (9 and 12 months only for intervention group). Furthermore, adherence, used as a proxy for concordance, was monitored using the MEMS. The questionnaires included in the study were Epilepsy Self-Efficacy Scale-33 item (ESES), the Quality of Life in Epilepsy-patient-weighted (QOLIE-31-P), the EuroQol 5 dimensions 5 levels (EQ-5D-5L), and a questionnaire regarding (health care) resource use over the past 3 months.

The ESES was used to assess the efficacy of self-management education program on epilepsy. Self-efficacy was defined as the confidence to carry out a behavior necessary to reach a desired goal. ${ }^{19-21}$ The items represent three dimensions of self-management: medication management, seizure management, and general management including safety and health. The total possible scores for the ESES range from 0 to 330 . Higher scores correspond to higher levels of confidence in ability to manage epilepsy. ${ }^{22}$

The EQ-5D-5L was used to assess generic QoL expressed in a utility with a value between 0 and 1 , where 1 means perfect health and 0 worst imaginable state of health. ${ }^{23}$ Utilities were derived using the Dutch tariffs. ${ }^{24}$

Disease-specific quality of life was measured with the QOLIE-3 1-P, which consists of 38 items assessing 7 domains of epilepsy: seizure worry, overall $Q O L$, emotional wellbeing, energy-fatigue, cognitive functioning, medication effects, social functioning and an overall score. ${ }^{25}$ The final score ranges from 0 to 100 , in which higher values indicate a better QoL. ${ }^{26}$ 
Health care resource use was measured using the Medical Cost Questionnaire, which was an adapted version of the Trimbos/iMTA questionnaire for costs associated with psychiatric illness. Productivity costs were measured with the Productivity Cost Questionnaire, both covering 3 months.

A detailed description of the measurement instruments can be found in Leenen \& Wijnen et al. (2014)..$^{16}$

\section{Costs}

This economic evaluation was performed according to the Dutch guidelines for economic evaluations $^{27}$ and the Consolidated Health Economic Evaluation Reporting Standards. ${ }^{28} \mathrm{~A}$ cost-effectiveness (CEA) and cost-utility analysis (CUA) were performed from a societal perspective to estimate the cost per QALY gain.

Costs were divided into four cost categories: intervention costs, healthcare sector costs, costs for patient and family, and productivity costs. The calculation was based on a detailed inventory covering all cost items composed of standardized cost prices from the Dutch manual for costing, and the calculated mean cost prices from the providers. To determine the costs of drugs, the website of the Dutch healthcare institute for the cost of pharmaceuticals (www.mediciinkosten.nl) was used. Intervention costs included the costs of the MEMS and costs associated with the $\mathrm{MCl}$ such as overhead costs, costs for instructors, costs of feedback sessions and time costs for patients and relatives or friends (if a relative or friend was brought to the group sessions by a patient). Healthcare costs consisted of consultations with healthcare professionals, the use of diagnostic methods, and the frequency of inpatient stay and outpatient treatment. Patient and family costs included the use of formal (paid) care, informal care, and medical devices. The costs for unpaid care were valued using the proxy good method, which values the time spent on informal care at the labor market price of a close market substitute. Productivity costs included productivity losses due to absence from work and were valued using the friction cost method as recommended in the Dutch guidelines. ${ }^{27}$ The friction cost method implies that long-term sick or absent employees will be replaced by employers after a specified friction period based on the average period an employer needs to replace a sick employee. In the Netherlands, a friction period of 85 days is recommended. All costs were indexed for the year 2015. As the study follow-up was one year, no discounting was performed (for either costs or effects).

\section{Analyses}

Baseline differences in costs were checked with nonparametric bootstrapping, based on 1,000 bootstrap replications, as the data did not comply with the underlying assumptions of parametric tests using Microsoft Excel 2010. ESES-scores, QOLIE-31P scores, utilities and demographic variables were assessed for baseline differences using independent t-tests (for continuous variables) or Pearsons' chi-square tests (for categorical variables) using IBM SPSS Statistics version 24. Adherence rates were compared using Wilcoxon 
rank test for repeated measurements. Missing values (i.e. EQ-5D-5L, ESES, QOLIE-3 $1 p$ and costs) at baseline were managed using mean imputation ( $\mathrm{n}=3$ for $\mathrm{CAU}$ group and $\mathrm{n}=1$ for intervention group) due to the low number of missing values and as no clinical information was available for these patients.

Missing data at follow-up measurements were dealt with using multiple imputation (5 times). Imputation was based on age, number of medications, employment, seizure frequency at baseline, seizure severity, self-efficacy score, randomization group, health care, patients \& family and productivity costs at each time point (for cost data only), EQ-5D-5L scores at each time point (for EQ-5D data only), ESES scores at each time point (for ESES data only) and QOLIE-3 1 p score at each time point (for QOLIE-3 $1 p$ data only). To account for non-normality of the cost data, predictive mean matching was used in which "real" observed values from similar cases were imputed instead of imputing regression estimates. ${ }^{29,30}$ QALYs were calculated by means of the "under the curve method," in which the time in a certain health state was multiplied by the utility of this health state. ${ }^{31}$

Disease-specific QALYs were calculated based on the QOLIE-3 1 p by dividing the total QOLIE-3 1 p score by 100 to obtain disease-specific utility-like scores.

An intention to treat (ITT) protocol was used, meaning that all patients initially included in the study were included in the analyses. For the analysis of the MEMS, this implied that all patients who initiated the MEMS were included in the analysis. Analyses were carried out at 6 months (end of comparative phase) and after 12 months' follow-up. The CAU group was only followed for 6 months and the results extrapolated to 12 months assuming that this period was sufficient to obtain a representative estimation of 12 months follow-up costs. As adherence was measured using MEMS caps in both groups, which may be seen as an intervention in both groups, the ICER for adherence rates was only calculated for the comparative phase (6 months).

Incremental cost-effectiveness ratios (ICERs) and cost-utility ratios (ICURs) were calculated by dividing the incremental costs by the incremental effects or QALYs, resulting in the costs per increased adherence, self-efficacy, QALY or disease-specific QALY of the $\mathrm{MCl}$ as opposed to CAU. To quantify the uncertainty around the ICER/ICUR, nonparametric bootstrapping was performed (5000 times). To show the probability that the $\mathrm{MCl}$ is cost-effective, given different ceiling ratios, a cost-effectiveness acceptability curve (CEAC) was constructed.

In the Netherlands, the Council for Public Health and Health Care proposed an informal ceiling ratio for QALYs between $€ 20,000$ and $€ 80,000$ per QALY, depending on the burden of disease. ${ }^{32}$ Based on this proposition and the disability weight of (severe) epilepsy reported elsewhere, ${ }^{33}$ one could assume that the maximum willingness to pay for one QALY is approximately $€ 50,000$.

A regression-based adjustment was performed to account for the difference in baseline costs, (disease-specific) utilities and ESES scores between both groups. ${ }^{34,35}$

One-way sensitivity analyses were performed by 1) Using disease-specific QALYs based on the QOLIE-31p; 2) applying a healthcare perspective, instead of a societal perspective, as some European countries tend to adopt this; 3) excluding the costs of the 
MEMS for the CAU group as patients in the CAU group did not receive any feedback from the MEMS and simply used the device for research purposes; and 4) by using the UK tariff to value the EQ-5D-5L health states.

\section{Results}

\section{Baseline characteristics}

A total of 102 patients were included in the study, 52 of whom were in the intervention group. A large part of the patients reported to have an unpaid job or to be unemployed (61.8\%). There was, however, a significant difference between the intervention group and the CAU group, indicating the intervention group had significantly more patients who had a paid job $(p=0.02)$. Furthermore the intervention group had a significantly higher score on the ESES $(p=0.002)$. Apart from employment rate and ESES score, no other baseline differences were found (see Table 10.1).

At baseline, health care costs and patient \& family costs were similar between both groups. Productivity costs were, however, higher in the intervention group, leading to higher costs overall in the intervention group compared to the CAU group (i.e. €3,338 versus $€ 2,030)$. Although the two $(95 \%)$ confidence intervals of total costs overlap marginally, a baseline correction was made. Unit prices are presented in Supplementary Material S10.1.

\section{Clinical effectiveness}

There was a significant difference at baseline regarding ESES-scores $(p=0.024)$. Hence, significance testing at follow-up was performed based on mean change scores; this did not reveal significant differences between groups at 3 or 6 months' follow-up.

Mean adherence rates over 6 months, as measured with the MEMS, were $63.7 \%$ for the CAU group and $75.9 \%$ for the intervention group, which was not shown to be a significant difference. Adherence decreased slightly over time, resulting in an adherence rate of $71.05 \%$ at 12 months for the intervention group. In total, $18 \%$ of the subjects in CAU group and $4 \%$ of the subjects in the intervention group did not initiate the MEMS cap and were excluded from the analyses (resulting in 94 patients, 44 CAU group and 49 intervention group). Patients who were lost to follow-up or who did not used the MEMS caps were considered as non-adherent. Utility scores derived from the EQ-5D-5L did not show significant differences at any of the follow-up measurements. Total QALYs at 6 months were 0.41 for both the intervention group and the control group using Dutch tariffs. 
Table 10.1 (Baseline) characteristics of the population

\begin{tabular}{|c|c|c|c|}
\hline Characteristics & $\begin{array}{l}\text { Intervention group } \\
(n=52)\end{array}$ & $\begin{array}{l}\text { Control group } \\
(n=50)\end{array}$ & $\begin{array}{c}\text { Total } \\
(n=102)\end{array}$ \\
\hline \multicolumn{4}{|l|}{ Gender } \\
\hline Male & $28(53.9 \%)$ & $22(44 \%)$ & $50(49.0 \%)$ \\
\hline Female & $24(46.2 \%)$ & $28(56 \%)$ & $52(51.0 \%)$ \\
\hline Age in years (mean) & 39.98 (SD: 13.1$)$ & 43.51 (SD: 15.4) & 41.71 (SD: 14.7) \\
\hline $18-24$ & $7(13.5 \%)$ & $7(14 \%)$ & $14(13.7 \%)$ \\
\hline $25-44$ & $23(44.2 \%)$ & $20(40 \%)$ & $43(42.2 \%)$ \\
\hline $45-64$ & $21(40.4 \%)$ & $18(36 \%)$ & $39(38.2 \%)$ \\
\hline$\geq 65$ & $1(1.9 \%)$ & $5(10 \%)$ & $6(5.9 \%)$ \\
\hline \multicolumn{4}{|l|}{ Marital status, $n(\%)$} \\
\hline Married/Cohabiting & $24(46.1 \%)$ & $28(56.0 \%)$ & $52(51.0 \%)$ \\
\hline Living alone & $15(28.8 \%)$ & $11(22.0 \%)$ & $26(25.5 \%)$ \\
\hline Living with parents & $7(13.5 \%)$ & $8(16.0 \%)$ & $15(14.7 \%)$ \\
\hline Other & $3(5.8 \%)$ & $1(2.0 \%)$ & $4(3.9 \%)$ \\
\hline Missing values & 3 & 2 & $5(4.9 \%)$ \\
\hline \multicolumn{4}{|l|}{ Education, n (\%) } \\
\hline No education & $2(3.8 \%)$ & $4(8.0 \%)$ & $6(5.9 \%)$ \\
\hline Primary school & $1(1.9 \%)$ & $3(6.0 \%)$ & $4(3.9 \%)$ \\
\hline Pre-vocational secondary school & $29(55.8 \%)$ & $30(60.0 \%)$ & $59(57.8 \%)$ \\
\hline Secondary School ${ }^{a}$ & $6(11.5 \%)$ & $3(6.0 \%)$ & $9(8.8 \%)$ \\
\hline Higher education $^{\mathrm{b}}$ & $10(19.2 \%)$ & $5(10.0 \%)$ & $15(14.7 \%)$ \\
\hline Other & $2(3.8 \%)$ & $3(6.0 \%)$ & $5(4.9 \%)$ \\
\hline Missing values & 2 & 2 & $4(3.9 \%)$ \\
\hline \multicolumn{4}{|l|}{ Daily life, $n(\%)^{c}$} \\
\hline Study & $6(11.5 \%)$ & $3(6.0 \%)$ & $9(8.8 \%)$ \\
\hline Work & $20(38.5 \%)$ & $11(22.0 \%)$ & $31(30.4 \%)$ \\
\hline Entrepreneur & $4(7.7 \%)$ & 0 & $4(3.9 \%)$ \\
\hline Housewife/houseman & $3(5.8 \%)$ & $5(10.0 \%)$ & $8(7.8 \%)$ \\
\hline Unemployed & $9(17.3 \%)$ & $7(14.0 \%)$ & $16(15.7 \%)$ \\
\hline Incapacitated & $5(9.6 \%)$ & $16(32.0 \%)$ & $21(20.6 \%)$ \\
\hline Retirement or early retirement & $2(3.8 \%)$ & $4(8.0 \%)$ & $6(5.9 \%)$ \\
\hline Other & $1(1.9 \%)$ & $2(4.0 \%)$ & $3(2.9 \%)$ \\
\hline Missing values & $1(1.9 \%)$ & $2(4.0 \%)$ & $3(2.9 \%)$ \\
\hline \multicolumn{4}{|l|}{ Employment $^{c}$} \\
\hline Unpaid job / unemployed & $27(51.9 \%)$ & $36(72.0 \%)$ & $63(61.8 \%)$ \\
\hline Employed & $24(46.2 \%)$ & $11(22.0 \%)$ & $35(34.3 \%)$ \\
\hline Missing & $1(1.9 \%)$ & $3(6.0 \%)$ & $4(3.9 \%)$ \\
\hline $\begin{array}{l}\text { Mean number of seizures } 4 \text { weeks prior } \\
\text { baseline }\end{array}$ & 5.2 (SD: 13.2) & 4.5 (SD: 7.8) & \\
\hline Epilepsy Self-Efficacy Scale score ${ }^{c}$ & 243.8 (SD: 23.3) & 230.4 (SD: 32.3$)$ & \\
\hline \multicolumn{4}{|l|}{ Hospital Anxiety and Depression Scale } \\
\hline Total anxiety score & 6.5 (SD: 3.4) & $7.4(S D: 3.4)$ & \\
\hline Total depression score & 4.8 (SD: 3.2) & 5.2 (SD: 3.0 ) & \\
\hline \multicolumn{4}{|l|}{$\begin{array}{l}\text { Medication adherence monitoring system } \\
\text { (adherence rate) }\end{array}$} \\
\hline 6 months follow-up & $75.9 \%$ (SD: 32.5$)$ & $63.7 \%$ (SD: 36.6$)$ & \\
\hline 12 months follow-up & $71.0 \%$ (SD: 36.6$)$ & - & \\
\hline
\end{tabular}

a Includes: General vocational school, pre-university school and secondary vocational education; ${ }^{b}$ Includes: Professional and academic higher education; ${ }^{c}$ Sig. difference between groups at $5 \%$ level 
Although baseline QOLIE-31p scores differed, the difference was not significant between groups. However, at 3 months follow-up the intervention group had significantly higher QOLIE-3 $1 p$ scores ( $p=0.035)$. Total disease-specific QALYs, as derived from the QOLIE-31p, were significantly higher in the intervention group (0.34) compared to the CAU group (0.32) at 6 months' follow-up with a p-value of 0.041 , and at 12 months' follow-up $(p=0.048)$. Scores on the visual analogue were significantly different at 12 months' follow-up ( $p=0.024)$. When adjusting for baseline differences, no significant differences were found between groups. Table 10.2 presents a detailed overview of the clinical effectiveness data.

\section{Cost-effectiveness}

The intervention costs were $€ 422$ and $€ 648$ for the intervention group at 6 and 12 months' follow-up and $€ 95$ for the control group. The intervention costs for the control group were mainly protocol driven costs attributable to the MEMS bottle. At 6 months, total costs were higher for the intervention group $(€ 4,491)$ compared to the CAU group $(€ 4,142)$. At 12 months, total costs were $€ 9314$ for the intervention group and $€ 8189$ for the CAU group. Total costs at 12 months' follow-up adjusted for baseline differences were $€ 9014$ for the intervention group and $€ 8495$ for the CAU group (see Table 10.3).

Looking at the ESES, the combination of effects and costs resulted in an ICER of $€ 54$ per point increase in ESES-score at 6 months and $€ 1105$ per point increase in ESES score at 12 months' follow-up. Concerning adherence, the intervention resulted in an ICER of $€ 88$ per percentage of adherence increase at 6 months. When looking at the QALYs (Dutch tariff), an ICUR of $€ 8,272$ per QALY gain was found at 6 months' follow-up and an ICUR of $€ 15,144$ per QALY gained at 12 months' follow-up. Results of the bootstrap replications of the ICERs/ICURs are presented in Figure 10.2. This figure presents the differences in costs and effects between the $\mathrm{MCl}$ and $\mathrm{CAU}$ for each bootstrap replication (1000 times).

The probability that the $\mathrm{MCl}$ is cost-effective given different ceiling ratios, as presented in CEACs, is shown in figure 3. At a willingness to pay $€ 2,000$ per point increase on the ESES score, the probabilities of the $\mathrm{MCl}$ being cost-effective is $69 \%$ and $52 \%$ at 6 and 12 months' follow-up, respectively. In terms of QALYs, if one would be willing to pay $€ 50,000$ per QALY gained, the probability that the $\mathrm{MCl}$ would be cost-effective is $74 \%$ at 6 months follow-up and $76 \%$ at 12 months follow-up (see Figure 10.3). 


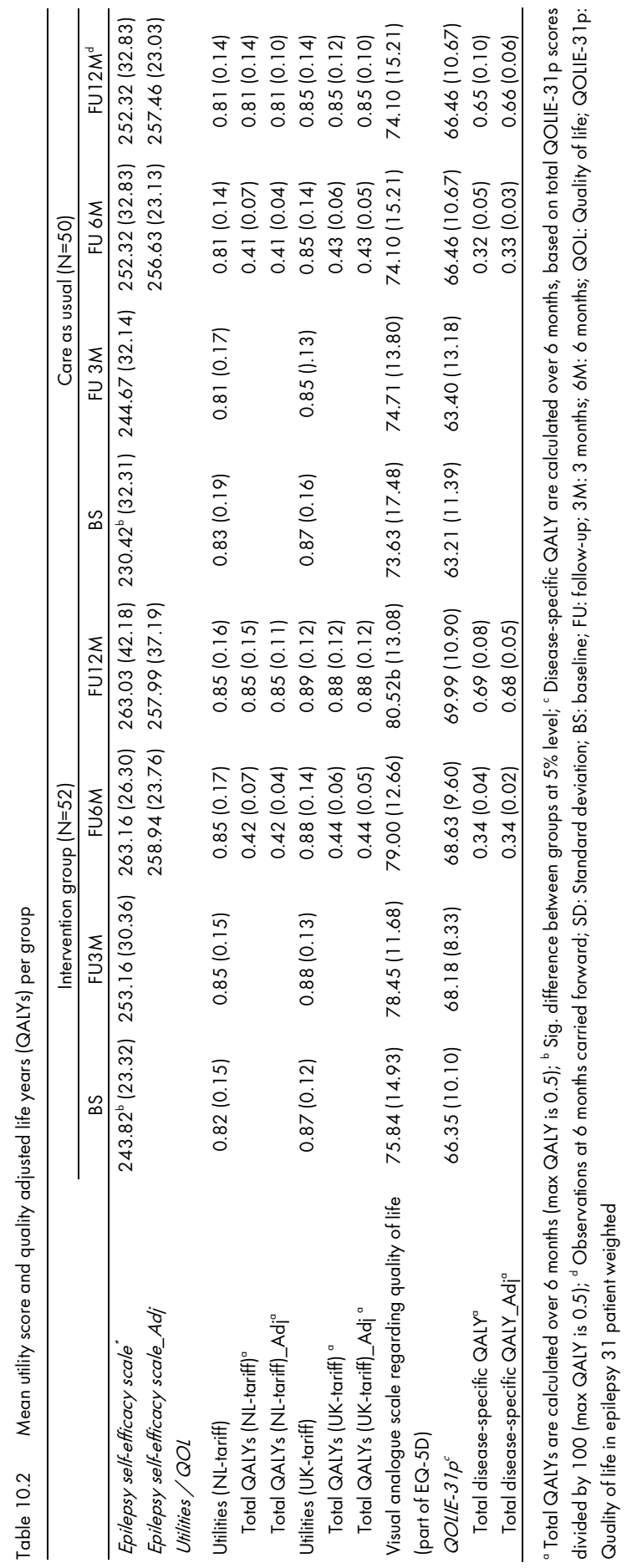




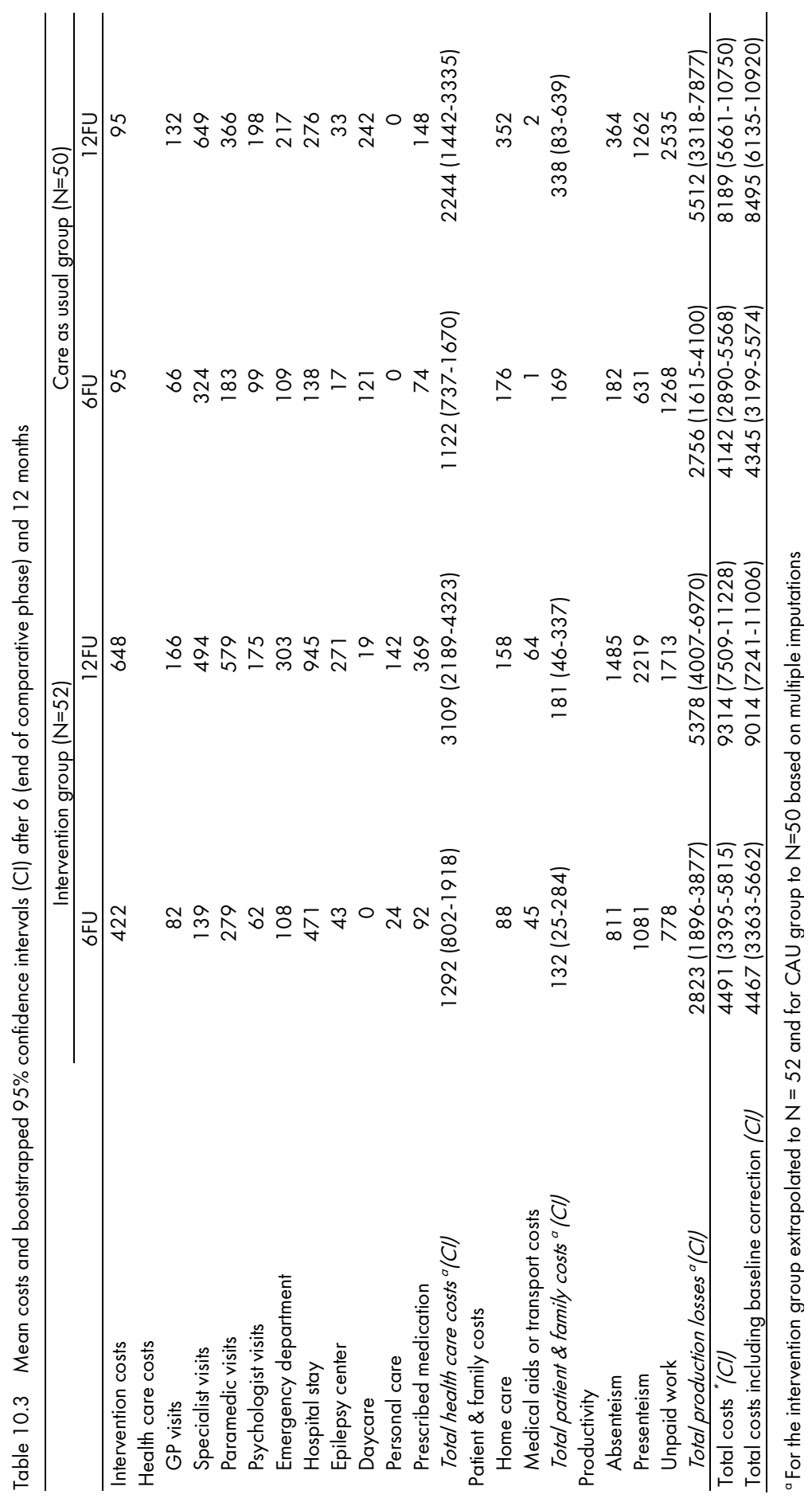




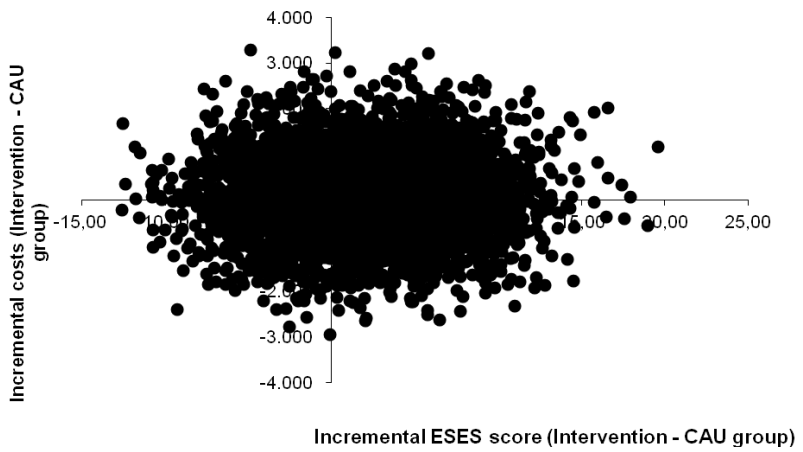

Figure 10.2a Cost-effectiveness plane of ESES scores at 6 months follow-up (costs per point ESES score increase)

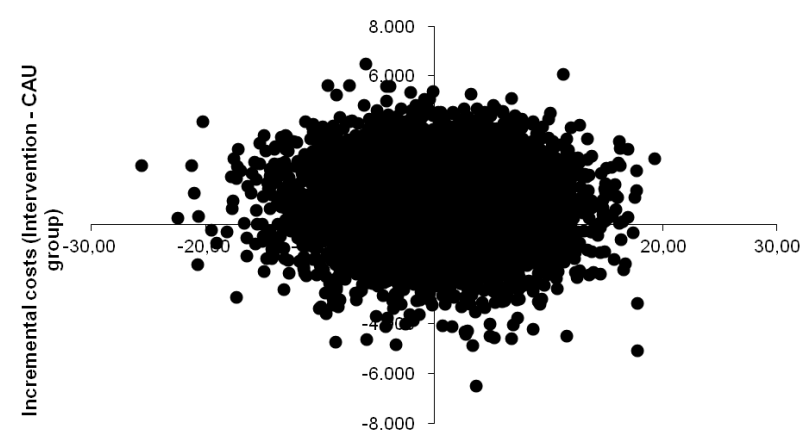

Incremental ESES score (Intervention - CAU group)

Figure 10.2b Cost-effectiveness plane of ESES scores at 12 months follow-up (costs per point ESES score increase)

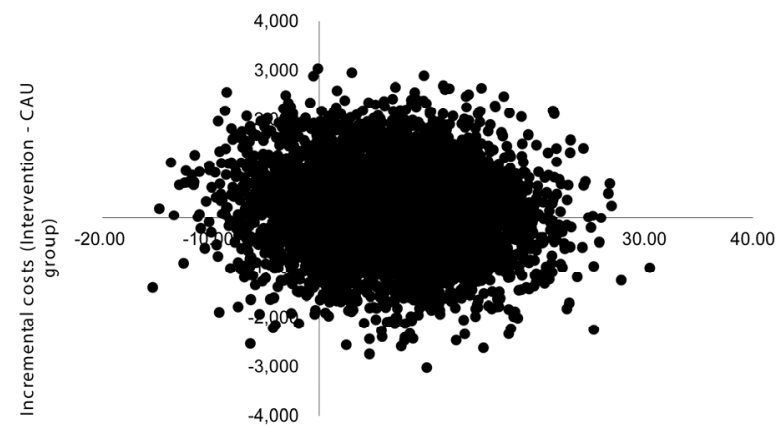

Incremental adhernce (Intervention - CAU group)

Figure 10.2c Cost-effectiveness plane of percentage adherence at 6 months follow-up (costs per percentage increase in adherence increase) 


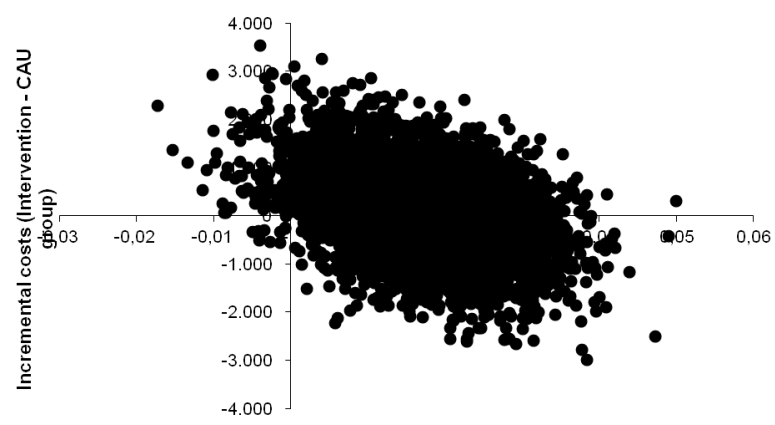

Incremental QALY (Intervention - CAU group)

Figure 10.2d Cost-effectiveness plane of QALY scores at 6 months follow-up (costs per QALY gained)

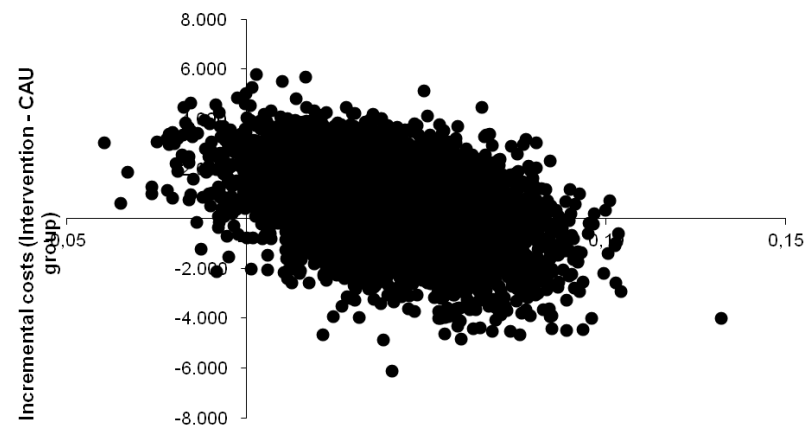

Incremental QALY (Intervention - CAU group)

Figure 10.2e Cost-effectiveness plane of QALY scores at 12 months follow-up (costs per QALY gained)

\section{Sensitivity analyses}

Disease-specific QALY: the ICURs at 6 and 12 months' follow-up, respectively, were $€ 15,975$ and $€ 36,824$ per disease-specific QALY gained. Applying a healthcare perspective instead of a societal perspective led to ICERs of $€ 214$ and $€ 2,034$ per score improvement on ESES score at 6 and 12 months, respectively. Looking at adherence, an ICER of $€ 65$ per percentage increase in adherence was found at 6 months. In terms of QALYs, the healthcare perspective led to an ICUR of $€ 31,502$ per QALY gained at 6 months' follow-up and $€ 27,850$ per QALY gained at 12 months' follow-up.

Excluding the costs of the MEMS for the CAU group resulted in an ICER of €93 per point increase in ESES-score at 6 months, $€ 1,546$ per point increase in ESES score at 12 months follow-up and in ICER of $€ 100$ per percentage adherence increase. Excluding the cost of MEMS for the CAU resulted in an ICUR of $€ 12,901$ per QALY gained at 6 months' follow-up, and €20,363 per QALY gained at 12 months' follow-up. 


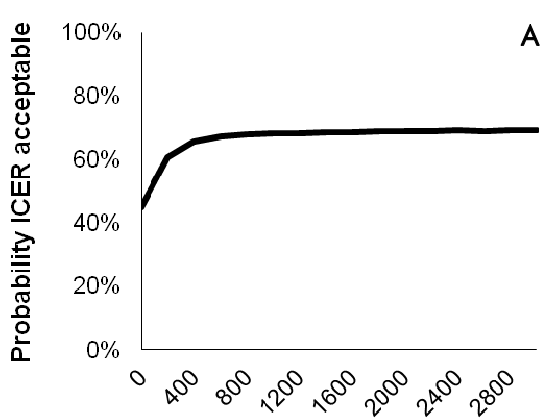

Limit on ICER
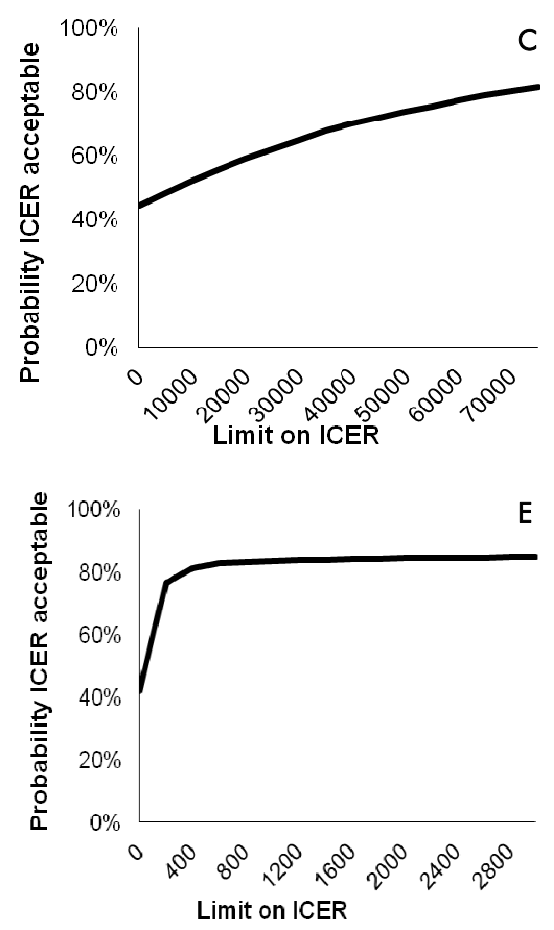

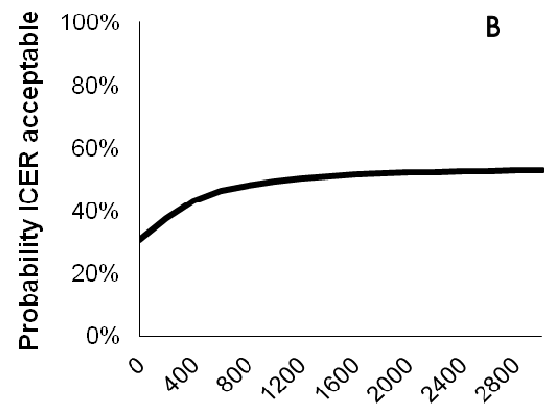

Limit on ICER

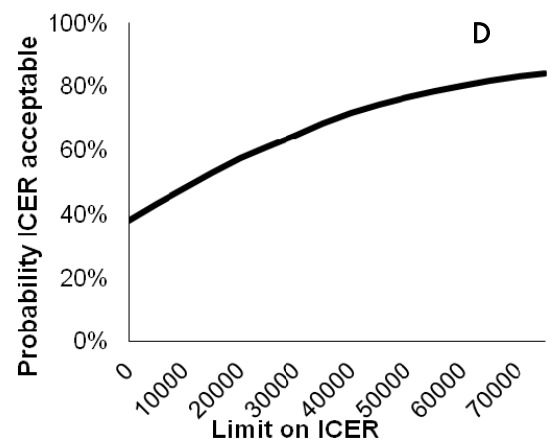

Figure 10.3 A. Cost-effectiveness acceptability curve of costs per ESES score improved at 6 months; B. costeffectiveness acceptability curve of costs per ESES score improved at 12 months; C. Costeffectiveness acceptability curve of costs per QALY gained at 6 months; D. cost-effectiveness acceptability curve of costs per QALY gained at 12 months; E. cost-effectiveness acceptability curve of costs per percentage increase in adherence at 6 months 
Using the UK tariffs to value the EQ-5D-5L health states resulted in ICURs of $€ 9,983$ and $€ 16,658$ per QALY gained at 6 and 12 months' follow-up, respectively.

Lastly, the analyses were performed without baseline adjustments. This resulted in an ICER of $€ 48$ per score improvement on ESES score at 6 months, an ICER of $€ 105$ per score improvement on ESES score at 12 months, and an ICER of $€ 147$ per percentage increase in adherence. In terms of QALYs, this led to an ICUR of $€ 30,989$ per QALY gained at 6 months' follow-up and $€ 36,585$ per QALY gained at 12 months' follow-up.

\section{Discussion}

Cost-effectiveness estimates appeared to be promising. Unlike, QALYs, there is no willingness to pay threshold known for improvement on the ESES score, which makes it difficult to draw firm conclusions regarding cost-effectiveness. Regarding QALYs, considering a threshold of $€ 50,000$ per QALY gained, the probability that the $\mathrm{MCl}$ was cost-effective was estimated to be $74 \%$ at 6 months' follow-up and $76 \%$ at 12 months' follow-up.

The $\mathrm{MCl}$ did not lead to significantly higher levels of self-efficacy. Furthermore, EQ-5D-5L score did not differ between the two groups. Total costs were higher for the intervention group compared to the control group.

The use of baseline corrections impacted the results as demonstrated by the sensitivity analyses. However, the imbalances in important baseline characteristics made us consider adjusting for them. It has been argued that imbalances in baseline measures could in fact matter without being statistically significant ${ }^{36}$ and that baseline adjustments provide more precise estimates of a treatment effect. ${ }^{35}$ Furthermore, although in contrast to, for example, QALYs, baseline costs are not part of the total costs, one could still expect that patients' baseline costs will influence costs during the study. Hence, it is important to investigate this influence. $^{34}$

The lack of effectiveness of the $\mathrm{MCl}$ on the ESES and EQ-5D-5L may be explained by several factors. Of course, it could be that the intervention is not effective. However, this may be too short-sighted. For example, it is also possible, given the nature of the intervention, that we simply did not capture the effect or that the instruments were not sufficiently responsive. The first argument applies mainly to the ESES, as self-efficacy has been shown to increase in other studies investigating self-management programs. ${ }^{37}$ The latter argument applies in particular to the EQ-5D-5L, which has previously been reported not to be responsive in patients with epilepsy. ${ }^{4}$ However, it has been argued that rules of inference are arbitrary and irrelevant to the decisions which clinical and economic evaluations claim to inform. Claxton et al. (1999) argued that, if the objective is to maximize health gain for a given budget, decisions should be based only on the mean net benefits irrespective of whether differences are statistically significant. ${ }^{38}$ This is because one of the mutually exclusive alternatives must be chosen and this decision cannot be 
postponed. By accepting the arbitrary rules of inference, one will impose costs which can be measured in terms of resources or lost health benefits. ${ }^{38}$

This study is not without its limitations. First, a $\mathrm{MCl}$ tailored to a specific age-group may be more effective. For example, it has been shown that adults with lower education and income may be poorer self-managers than those with higher income and/or educational levels. ${ }^{39}$ Second, due to the fact that the interventions consist of multiple components, it is difficult to determine which aspect contributed to the effects found in this study. In addition, although the CAU group did not receive feedback on their usage, both the CAU and the intervention group received the MEMS which means CAU deviated from standard care. Third, patients were instructed to use the smartphone application "Eppy" (Epilepsy Foundation, the Netherlands). Due to financial constraints, Eppy was not maintained and updated, which meant that only a minority of the patients were able to use the app. Moreover, it was discontinued in early 2015 and removed from both the App store (Apple Inc., USA) and the Google Play store (Google Inc., USA). Fourth, the sample size of this study was based on a standard deviation for the ESES score of 7 points. However, the actual standard deviation found in this study was $>30$ points, which may have resulted in a decrease in study power. This may be a reason why we did not find statistically significant results. Lastly, with regard to the 12 month sensitivity analyses, the CAU group was only followed for 6 months and the results extrapolated to 12 months. This implies that the 6-month follow-up of the CAU group was sufficient to obtain a representative estimation of 12 month follow-up costs.

In conclusion, although there was no statistically significant difference found after baseline adjustments, cost-effectiveness estimates appear to be promising. It has been argued that rules of inference are arbitrary and perhaps less relevant to the decisions which clinical and economic evaluations aim to inform. Further research should try to determine which factors contribute to an increased QoL in patients with epilepsy. 


\section{References}

1. Strzelczyk A, Reese JP, Dodel R, Hamer HM. Cost of epilepsy: a systematic review. Pharmacoeconomics. 2008;26(6):463-76.

2. (RIVM) HRvVeM. Een gezonder Nederland met meer chronisch zieken. RIVM, RIVM. 2014.

3. Allers K, Essue BM, Hackett ML, Muhunthan J, Anderson CS, Pickles K et al. The economic impact of epilepsy: a systematic review. BMC Neurol. 2015;15:245.

4. de Kinderen RJ, Lambrechts DA, Wijnen BF, Postulart D, Aldenkamp AP, Majoie MH et al. An economic evaluation of the ketogenic diet versus care as usual in children and adolescents with intractable epilepsy: An interim analysis. Epilepsia. 2016;57(1):41-50.

5. Strzelczyk A, Reese JP, Dodel R, Hamer HM. Cost of Epilepsy. PharmacoEconomics. 2008;26(6):463-76.

6. Annemans L. Gezondheidseconomie voor niet-economen: Een inleiding tot de begrippen, methoden en valkuilen van de gezondheidseconomische evaluatie. Academia Press; 2007.

7. Williams I, Mclver S, Moore D, Bryan S. The use of economic evaluations in NHS decision-making: a review and empirical investigation. Health technology assessment (Winchester, England). 2008; 12(7):iii, ix-x, $1-175$.

8. ZorginstituutNL. Economische evaluatie. https://www.zorginstituutnederland.nl/pakket/werkwijze+ pakketbeheer/beoordeling+geneesmiddelen/economische+evaluatie.

9. Samsonsen C, Reimers A, Brathen G, Helde G, Brodtkorb E. Nonadherence to treatment causing acute hospitalizations in people with epilepsy: an observational, prospective study. Epilepsia. 2014;55(11):e125-8.

10. Bodenheimer T, Lorig K, Holman H, Grumbach K. Patient self-management of chronic disease in primary care. JAMA. 2002;288(19):2469-75.

11. Bourbeau J, Collet JP, Schwartzman K, Ducruet T, Nault D, Bradley C. Economic benefits of selfmanagement education in COPD. Chest. 2006;130(6):1704-11.

12. Shegog R, Bamps YA, Patel A, Kakacek J, Escoffery C, Johnson EK et al. Managing Epilepsy Well: Emerging e-Tools for epilepsy self-management. Epilepsy Behav. 2013;29(1):133-40.

13. Cramer JA, Glassman M, Rienzi V. The relationship between poor medication compliance and seizures. Epilepsy Behav. 2002;3(4):338-42.

14. Amir M, Roziner I, Knoll A, Neufeld MY. Self-efficacy and social support as mediators in the relation between disease severity and quality of life in patients with epilepsy. Epilepsia. 1999;40(2):216-24.

15. Haynes RB, Ackloo E, Sahota N, McDonald HP, Yao X. Interventions for enhancing medication adherence. Cochrane Database Syst Rev. 2008(2):CD000011.

16. Leenen LA, Wijnen BF, de Kinderen RJ, Majoie MH, van Heugten CM, Evers SM. (Cost)-effectiveness of a multi-component intervention for adults with epilepsy: study protocol of a Dutch randomized controlled trial (ZMILE study). BMC Neurol. 2014;14:255.

17. Aspinwall LG, Taylor SE. A stitch in time: self-regulation and proactive coping. Psychol Bull. 1997; $121(3): 417-36$.

18. Thoolen B, de Ridder D, Bensing J, Gorter K, Rutten G. Beyond Good Intentions: the development and evaluation of a proactive self-management course for patients recently diagnosed with type 2 diabetes. Health Educ Res. 2008;23(1):53-61.

19. Lorig K, Gonzalez VM, Ritter P. Community-based Spanish language arthritis education program: a randomized trial. Med Care. 1999;37(9):957-63.

20. Lorig KR, Holman H. Self-management education: history, definition, outcomes, and mechanisms. Ann Behav Med. 2003;26(1):1-7.

21. Lorig KR, Mazonson PD, Holman HR. Evidence suggesting that health education for self-management in patients with chronic arthritis has sustained health benefits while reducing health care costs. Arthritis Rheum. 1993;36(4):439-46.

22. Dilorio C, Yeager K. The epilepsy self-efficacy scale. Teoksessa: Strickland OL \& Dilorio C (toim) Measurement of nursing outcomes: Self Care and Coping, New York. 2003:40-51.

23. Herdman $M$, Gudex C, Lloyd A, Janssen M, Kind P, Parkin D et al. Development and preliminary testing of the new five-level version of EQ-5D (EQ-5D-5L). Qual Life Res. 2011;20(10):1727-36.

24. Versteegh MM, Vermeulen KM, Evers SM, de Wit GA, Prenger R, Stolk EA. Dutch Tariff for the Five-Level Version of EQ-5D. Value Health. 2016;19(4):343-52. 
25. Cramer JA, Van Hammee G. Maintenance of improvement in health-related quality of life during long-term treatment with levetiracetam. Epilepsy Behav. 2003;4(2):1 18-23.

26. Devinsky O, Vickrey BG, Cramer J, Perrine K, Hermann B, Meador K et al. Development of the quality of life in epilepsy inventory. Epilepsia. 1995;36(11):1089-104.

27. Nederland Z. Richtlijn voor het vitvoeren van economische evaluaties in de gezondheidszorg. Diemen: Zorginstituut Nederland. 2015.

28. Husereau D, Drummond M, Petrou S, Carswell C, Moher D, Greenberg D et al. Consolidated health economic evaluation reporting standards (CHEERS) statement. Cost Eff Resour Alloc. 2013;1 1(1):6.

29. Grittner U, Gmel G, Ripatti S, Bloomfield K, Wicki M. Missing value imputation in longitudinal measures of alcohol consumption. Int J Methods Psychiatr Res. $2011 ; 20(1): 50-61$.

30. Horton NJ, Lipsitz SR. Multiple imputation in practice: comparison of software packages for regression models with missing variables. The American Statistician. 2001;55(3):244-54.

31. Drummond MF, Sculpher MJ, Claxton K, Stoddart GL, Torrance GW. Methods for the economic evaluation of health care programmes. Oxford university press; 2015.

32. Council for Public Health and Health Care. Sensible and sustainable care (in Dutch). Zoetermeer2006.

33. Salomon JA, Vos T, Hogan DR, Gagnon M, Naghavi M, Mokdad A et al. Common values in assessing health outcomes from disease and injury: disability weights measurement study for the Global Burden of Disease Study 2010. Lancet (London, England). 2012;380(9859):2129-43.

34. van Asselt $A D$, van Mastrigt GA, Dirksen CD, Arntz A, Severens JL, Kessels AG. How to deal with cost differences at baseline. Pharmacoeconomics. 2009;27(6):519-28.

35. Manca A, Hawkins N, Sculpher MJ. Estimating mean QALYs in trial-based cost-effectiveness analysis: the importance of controlling for baseline utility. Health Econ. 2005; 14(5):487-96.

36. Pocock SJ, Assmann SE, Enos LE, Kasten LE. Subgroup analysis, covariate adjustment and baseline comparisons in clinical trial reporting: current practice and problems. Stat Med. 2002;21(19):2917-30.

37. Dilorio C, Bamps Y, Walker ER, Escoffery C. Results of a research study evaluating WebEase, an online epilepsy self-management program. Epilepsy Behav. 201 1;22(3):469-74.

38. Claxton K. The irrelevance of inference: a decision-making approach to the stochastic evaluation of health care technologies. J Health Econ. 1999; 18(3):341-64.

39. Escoffery C, Bamps Y, LaFrance Jr WC, Stoll S, Shegog R, Buelow J et al. Development of the Adult Epilepsy Self-Management Measurement Instrument (AESMMI). Epilepsy Behav. 2015;50:172-83. 


\section{Supplementary material 10.1}

\section{Overview of the most important costs categories ZMILE study}

\begin{tabular}{|c|c|c|}
\hline Care type & $\begin{array}{c}\text { Unit price } \\
\text { (in euros; 2015) }\end{array}$ & Source \\
\hline \multicolumn{3}{|l|}{ Intervention costs ( 12 months) } \\
\hline Mean costs course (per patient) & 91 & (Calculated from) respective providers or professional organizations. \\
\hline MEMS cap with LCD & 86 & (Calculated from) respective providers or professional organizations. \\
\hline Feedback every 3 months & 452 & (Calculated from) respective providers or professional organizations. \\
\hline Other & 18 & (Calculated from) respective providers or professional organizations. \\
\hline \multicolumn{3}{|l|}{ Costs CAU group ( 12 months) } \\
\hline MEMS cap without LCD & 77 & (Calculated from) respective providers or professional organizations. \\
\hline Other & 18 & (Calculated from) respective providers or professional organizations. \\
\hline \multicolumn{3}{|l|}{ Health care costs } \\
\hline GP & 33 & Dutch guidelines for costing studies. \\
\hline Specialists visit & 91 & Dutch guidelines for costing studies. \\
\hline Social worker & 65 & Dutch guidelines for costing studies. \\
\hline Paramedic visits & 33 & Dutch guidelines for costing studies. \\
\hline Physiotherapist visit & 33 & Dutch guidelines for costing studies. \\
\hline Occupational therapist & 33 & Dutch guidelines for costing studies. \\
\hline Speech therapist visit & 30 & Dutch guidelines for costing studies. \\
\hline Dietician visit & 31 & (Calculated from) respective providers or professional organizations. \\
\hline Psychologist visits & 94 & Dutch guidelines for costing studies. \\
\hline Psychiatrist visit & 94 & Dutch guidelines for costing studies. \\
\hline Company doctor & 125 & (Calculated from) respective providers or professional organizations. \\
\hline Personal care (hour) & 23 & Dutch guidelines for costing studies. \\
\hline Home care (hour) & 73 & Dutch guidelines for costing studies. \\
\hline Emergancy department visit & 259 & Dutch guidelines for costing studies. \\
\hline Ambulance transport & 515 & Dutch guidelines for costing studies. \\
\hline Outpatient visit general hospital & 91 & Dutch guidelines for costing studies. \\
\hline Outpatient visit epilepsy center & 96.5 & Dutch guidelines for costing studies. \\
\hline Hospital stay, average (day) & 476 & Dutch guidelines for costing studies. \\
\hline Epilepsy center & 632 & (Calculated from) respective providers or professional organizations. \\
\hline 24h EEG registration & 823 & (Calculated from) respective providers or professional organizations. \\
\hline MRI & 207 & Dutch guidelines for costing studies. \\
\hline CT-scan & 130 & Dutch guidelines for costing studies. \\
\hline EEG scan & 236 & (Calculated from) respective providers or professional organizations. \\
\hline Medication & Variable & $\underline{\text { Www.mediciinkosten.nl }}$ \\
\hline \multicolumn{3}{|l|}{ Patient and family costs } \\
\hline Housekeeping (hour) & 23 & Dutch guidelines for costing studies. \\
\hline Transport (per kilometer) & 0.19 & Dutch guidelines for costing studies. \\
\hline Medical aids & Variable & (Calculated from) respective providers or professional organizations. \\
\hline \multicolumn{3}{|l|}{ Other costs } \\
\hline Absenteism & 34.98 & Dutch guidelines for costing studies. \\
\hline Presenteism & 34.98 & Dutch guidelines for costing studies. \\
\hline Unpaid work & 14.09 & Dutch guidelines for costing studies. \\
\hline
\end{tabular}



Discussion and summary
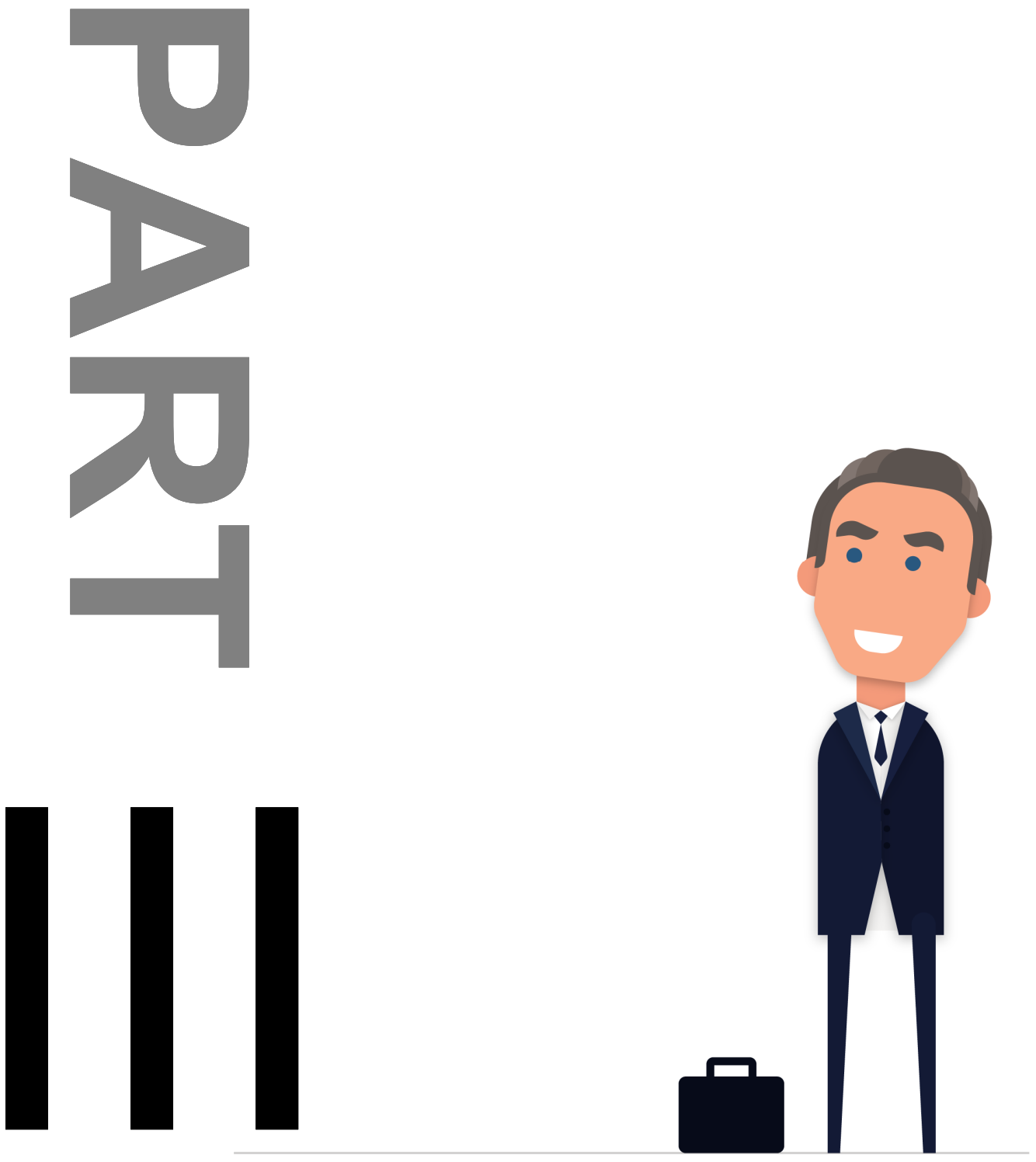



\section{General discussion}
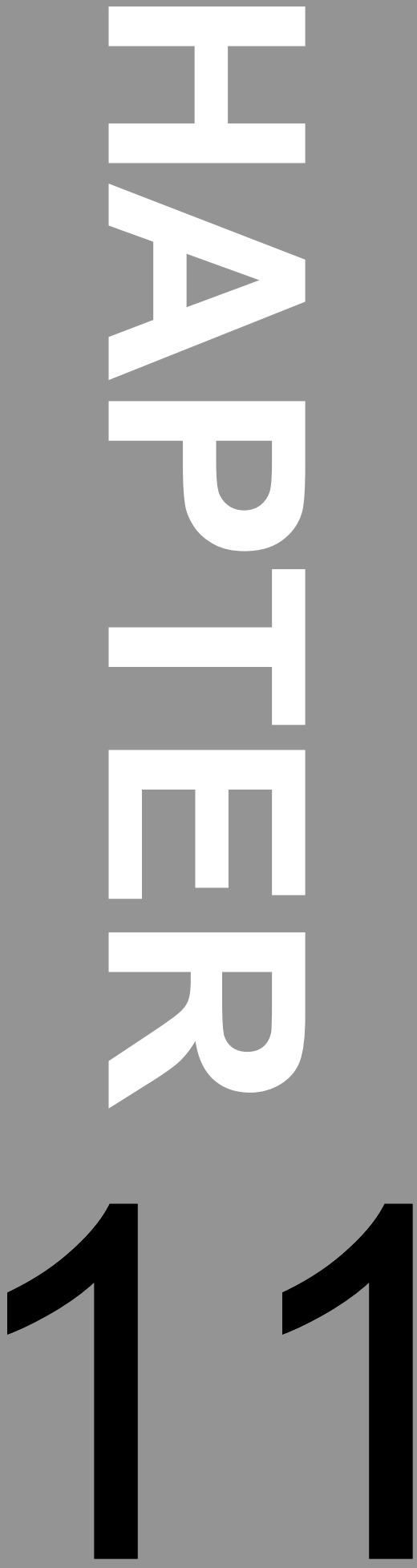



\section{General conclusion and discussion}

As described in the introduction, this thesis, which is structured around six research questions, consists of two main parts. The first part presents methodological aspects and preference-based studies of health technology assessment in epilepsy. The second part provides a more specific focus on economic evaluations within epilepsy. The present chapter opens with the main conclusions and answers to the research questions, with some elaboration, followed by a more detailed discussion of preference studies, selfmanagement and economic evaluations in epilepsy. Directions for further research are also indicated.

\section{Main results of the thesis}

\subsection{What preferences do people have regarding epilepsy diagnostics and the valuation of clinical manifestations of epilepsy?}

In chapter two, patients' preferences were obtained for brain imaging techniques in the primary diagnosis of epilepsy. Using a discrete-choice experiment (DCE), it was shown that patients attached importance to attributes other than psychometric properties (e.g. sensitivity; in this study, the chance of additional examination). For example, patients indicated preferring movement freedom during a registration. Using the relative importance weights for the attributes valued in this study, one could hypothetically compare different diagnostic modalities, for instance, MEG and EEG. Although MEG is often considered to be more patient friendly, it was demonstrated that this is not necessarily the case. Furthermore, the study shows that preferences could differ substantially between subgroups and even between individuals within a subgroup, given the considerable heterogeneity within the parameters. This highlights the importance of accounting for individual preferences in medical decision-making. ${ }^{\prime}$

In chapter three, preferences of the general public were obtained to determine utility values for several clinical manifestations of epilepsy, or for what is usually referred to as health states. This study provided a utility-function for epilepsy health states and gave insights into the relative impact of clinical outcomes in epilepsy on quality of life. Furthermore, it clearly demonstrated that seizure severity had a greater negative impact on utility compared with the attributes seizure frequency and side-effects. ${ }^{2}$

Although outcome valuations used in cost-utility analyses can be given by different groups, generally either patients or the general public (i.e. community preferences), which population should be preferred is still a matter of controversy One possible concern (with the use of community preferences) is patient-discrimination. ${ }^{3}$ This concern is supported by the prospect theory (PT) which states that people assess outcomes not according to an absolute standard, but rather according to a relative one, called a reference level. ${ }^{3}$ 
According to this theory, people allocate larger utilities for improvements near the reference level, but small utilities for improvements far from the reference level. ${ }^{3}$ Community preferences could, therefore, underestimate utility gains in people with a more severe health state, since the community is supposed to be in relatively good health. So the differences between pre- and post-treatment utilities would be larger for patients than for community members. This is especially the case when a treatment causes only a slight improvement in the patient's health status, which could mean a lot to the patient but does not seems to be of importance in the eyes of community.

On the other hand, community preferences could be distorted, because community fails to predict that a patient adapts to their health state. This phenomenon is called impact bias, which implies that people misinterpret the health state they are in when evaluating various health states. ${ }^{4}$ Impact bias could, therefore, cause an overestimation of utility gains in patients. This can also be explained by PT. Based on PT, we would expect that the measured utility for a disease state would be lower for the healthy population than for the sick population, because the healthy population has a higher reference level, and so any sick health state would be perceived as a loss. ${ }^{3}$

A review by Neumann et al. ${ }^{5}$ found that several studies showed that individuals afflicted with a specific disease tend to value their health state more highly than those who have not experienced the condition, although they also found studies reporting similarities in preferences between patients and non-patients. Internationally, however, health state valuation is mostly based on preferences of the general public; patients' preferences usually only involve (new) medications or a treatment which they will actually undergo. For example, valuation sets for the widely used EuroQol-5D-5L are based on community preferences ${ }^{6}$ and the same holds for the valuations sets of the SF-36 and HUI Mark II. ${ }^{7,8}$ Another frequently used argument for using community preferences is that societal resource allocation decisions should be made by appealing to population-based community values. ${ }^{9}$ However, in Sweden, patient preferences are preferred and included in their General Guidelines for Economic Evaluations from the Pharmaceutical Benefits Board. ${ }^{10,11}$

A recent review by Versteegh \& Brouwer on patient and general public preferences concluded that although the properties of both perspectives have been extensively discussed in the literature, there does not seem to be any conclusive or theoretically sound justification for disregarding either perspective, as has been argued before. In addition, they emphasize that health economic guidelines should require QALYs based on both patient and general public preferences. ${ }^{11}$

\subsection{How do generic quality of life instruments compare to epilepsy- specific quality of life instruments?}

In chapter four, we have compared a generic quality of life instrument with an epilepsyspecific quality of life instrument. Generic quality of life instruments are designed to be applicable across all diseases or conditions, across different medical interventions and across a wide range of populations. Condition-specific quality of life measures are 
designed to be relevant to a particular condition or state, in this case epilepsy. ${ }^{12}$ The use of generic instruments is important for policymakers; however, the lack of sensitivity of these instruments may raise concerns.

The aim of this study was to compare the responsiveness of the QOLIE-31p and the EQ-5D-5L in epilepsy and to predict EQ-5D-5L values based on QOLIE-3 1p scores by means of a mapping function. This study demonstrated moderate correlations between the two instruments when looking at the total scores. This may imply that both instruments are measuring the same concept only to some extent and impose conceptual differences as to what is actually measured. The EQ-5D-5L showed substantially high ceiling effects and was less responsive than the QOLIE-31p. Furthermore, this study attempted to derive a mapping function which could be used in (future) economic evaluations to map QOLIE$31 \mathrm{p}$ data to EQ-5D-5L values. Such a mapping function would be useful for researchers who want to calculate quality-adjusted life years (QALYs) but who do not have results of a generic quality instrument (such as the EQ-5D-5L). Using different mapping functions, the highest adjusted $R^{2}$ we were able to regress was 0.265 using an OLS model with squared terms. This led to a mean absolute error of 0.103 . However, this adjusted $R^{2}$ value and the others found in this study were relatively small, which indicates a poor fit of the mapping models to predict EQ-5D-5L outcomes. Hence the usefulness of this mapping function in economic evaluations is questionable given the relatively large mean absolute error and poor model fit. This may stress the need for other approaches.

Generic quality of life instruments are important for determining the utility of a person which can then be used to calculate QALYs. A utility can be seen as expressing the value of a particular health state on a scale from 0 to 1 , where 0 represents "worst imaginable health state" and 1 "perfect health". Utilities can then be used to calculate quality adjusted life years (QALYs), by multiplying the utility of a health state by the time spent in that particular health state. Without going into much detail, utilities for most generic quality of life instruments are elicited according to the Multi Attribute utility Theory, an extension of the Expected Utility Theory of Von Neumann and Morgenstern. ${ }^{13}$ Three main axioms must be taken into account before it can be called a utility, namely, 1) the individual has transitive preferences; 2) the individual agrees that the decision problem is unchanged by substituting one outcome by an equally preferred outcome; and 3) the individual is able to express choices when uncertain. ${ }^{14}$ Hence, for example, the valuation set (or tariffs) is based on choice experiments (i.e. preferences) carried out in the general population in which utilities for all EQ-5D-5L health states are elicited by using time trade-off experiments (see chapter 2), discrete choice experiments (see chapter 1) or standard gamble approaches (not covered in this thesis). Hence, a preference-based measure is a questionnaire with a scoring function designed to weight the responses according to preferences for certain health conditions over others. ${ }^{15}$ Theoretically, if a condition-specific quality of life instrument were used for the calculation of QALYs, the valuation set should be constructed according to the same principles. As the QOLIE-31p is not constructed in this way, theoretically it cannot be used to calculate QALYs. Moreover, given the limited responsiveness, low correlations and the poor model fit of the mapping functions between 
the QOLIE-31p and the EQ-5D-5L, one could argue that there is a need for the development of condition-specific preference-based measures which can then be used to calculate condition-specific QALYs as a supplement to economic evaluations. ${ }^{16}$ The development of such condition-specific, preference-based quality of life instruments may ensure that the benefits of health-care interventions are adequately reflected in QALY estimates for economic evaluations not only in epilepsy but for all conditions. ${ }^{17}$

There is, however, a lack of guidance on how to deal with such condition-specific QALYs in the assessment of economic evaluations. In addition, some argue that such measures should only be considered when empirical evidence disproves the sensitivity of existing generic instruments and empirical evidence proves the superiority of the conditionspecific measure. ${ }^{15}$ Hence, it is clear that generic quality of life instruments have their limitations and that solely depending on current existing measures may not be the right way to evaluate health care interventions. Using preference-based condition specific measures may overcome some problems associated with generic measures, such as responsive issues, but lacks cross program comparability. Including both types of QALYs may be one way forward. ${ }^{16}$ Another option would be to derive well-conducted and validated mapping functions instead of developing condition-specific, preference-based measures, because these mapping functions supposedly to yield utility values comparable to the more frequently used generic instruments. ${ }^{15}$ As this study demonstrated, the second option is not always achievable. Lastly, other, more extensive, preference-based generic quality of life instruments, potentially useful in epilepsy, have been or are currently being developed, such as the AQoL-8D or the ICECAP. ${ }^{18,19}$

However, the psychometric properties of both instruments within the field of epilepsy have yet to be determined. Overall, more research is needed in this area as responsiveness issues may directly impact resource allocation decisions.

\subsection{To what extent is a novel method to elicit preferences used in health care?}

The past few decades have seen an increase in awareness of the value of shared decisionmaking between physicians and patients. There seems to be a greater need for a more patient-centered approach in delivering health care. Best-worst scaling (BWS) is a relatively novel method for eliciting preferences. The overview of BWS provided in chapter 5 sheds some light on its application in health care. ${ }^{20}$ It is important to point out that about two-thirds of studies were performed in the last two years. A total of 62 BWS cases were identified and to briefly recap, the three types were: 1) 26 BWS object cases, in which the researcher is interested in the relative values associated with each of a list of objects; 2) 29 BWS profile cases, in which the choice set has the structure of a single profile (multiple attributes). The respondent is, however, not required to consider the value of the profile as a whole, but has to consider the attribute-levels that describe it; and 3) 7 BWS multi-profile cases, which require a respondent to choose the worst (least attractive) profile as well as the best (most attractive) one in the current choice set. 
This increase in published BWS studies may indicate a greater awareness of advantages of the BWS method for eliciting preferences in health care. It is apparent that BWS, especially the object case, is capable of incorporating a larger set of items or factors - on average $17(M d n=12)$ - compared to DCE. In DCE, the average number of included factors was estimated to be 4 to $6 .^{21}$ BWS object case can be especially useful if one is interested in a list of items that is difficult to reduce to a number acceptable for other conjoint analyses, such as a DCE. Furthermore, compared to the DCE, BWS lespecially object and profile cases) may be less cognitively burdensome. For example, Potoglou et al. (2011) stated that best-worst tasks take advantage of an individual's propensity to identify and respond more consistently to extreme options and BWS tasks seem easier to understand. $^{22}$

It is important to bear in mind that the two methods are complementary. The goal of the study described in chapter 5 is merely to provide an overview of the application and current practices in healthcare, not to state that one method is superior. The strength of the DCE is, however, that it provides a comparison and trade-off of entire profiles, in contrast to BWS where respondents only focus on specific items or attributes. In a BWS multiprofile, respondents are also supposed to make a trade-off between entire profiles, but the need to elicit the best and worst alternative has the limitation of comparing a minimum of three profiles per choice task. Moreover, as there are only minor differences between BWS multi-profile case and DCE, the former has often been referred to as BWS DCE. ${ }^{23,24}$

This overview may increase awareness of BWS among researchers in the field of health technology assessment and provide insights into the various aims. However, although it presents an overview of current (and past) practices, these are in no way recommendations for best practices. As DCEs have been used in health care for a relatively long period compared to BWS, a considerable amount of methodological guidance has been developed, especially during the last decade. ${ }^{25-27}$ Some important methodological issues remain a challenge (e.g. sample size calculations), but most topics, such as designing and analyzing DCEs, have been elaborated on. Mühlbacher et al. have published a short series on theoretical foundations and methodological considerations when conducting a BWS (such as design issues), ${ }^{28,29}$ a guidance protocol has, however, yet to be developed. This would improve standardization and the comparability of BWS and may also enable more researchers to perform such studies.

\subsection{How to appraise economic evaluations and the applicability of an economic evaluation to a particular setting?}

Cost-effectiveness has become increasingly important within health care and hence, within epilepsy. For example, the updated version of the Dutch clinical practice guidelines for epilepsy has recently been extended to include economic evidence. ${ }^{30}$ When using economic evaluations for decision-making or clinical practice guideline (CPG) development, it is essential to determine their quality and applicability. For this purpose, many quality and transferability checklists have been developed over the years and one 
may not be able to see the wood for the trees. Chapter 6 offers some guidance in choosing appropriate checklists. In health economics, a subdivision is often made between partial and full economic evaluations. The term "full economic evaluation" refers to the comparative analysis of alternative courses of action in terms of both costs (resource use) and consequences (outcomes, effects). ${ }^{31}$ Full economic evaluations can vary in a variety of aspects, all of which can affect the quality of the evaluation and hence bias the results. Full economic evaluations are considered to be the best strategy for answering efficiency questions as both the costs and effects are derived from the same population. ${ }^{31}$ Consequently, most checklists focus solely on full economic evaluations. Partial economic evaluations are referred to as studies focusing only on costs and/or resource use. However, in (clinical practice) guideline development or policy-making, and/or in the absence of full economic evaluations, one might consider a partial economic evaluation (e.g. costs analysis or budget impact analysis). This may represent important an intermediate stage in our understanding of the costs and consequences of health services programs. $^{31}$

Similar to clinical studies, biases in (full) economic evaluations can occur as a result of poor methodological quality, which could impact the validity of the results in terms of generalizability or transferability. ${ }^{32}$ In chapter 6 , several risk of bias checklists have been evaluated. In general, it is concluded that, for full economic evaluations, one should use the $\mathrm{CHEC}$-extended ${ }^{33,34}$ or the BMJ checklist. ${ }^{35}$ Furthermore, in cases where one is specifically interested in model-based economic evaluations and if the expected number of included studies is low (e.g. $<10$ studies), the Philips checklist ${ }^{36}$ could be used. If the expected number of included model-based economic evaluations is high (e.g. >10 studies), the ISPOR checklist is likely to be more convenient. ${ }^{37}$ Lastly, to determine the transferability of a study (e.g. whether the results of a study are transferable to another country or setting), the Welte checklist should be used. ${ }^{38}$

These recommendations show that there is a difference in the assessment of trial-based economic evaluations and model-based economic evaluations. Also they imply that if one is willing to conduct a systematic review of economic evaluations, one should use at least two checklists to determine the risk of bias (given that one would include both model- and trial-based economic evaluations). By using two different checklists, quality scores between model- and trial-based economic evaluations are difficult to compare. Hence, as mentioned in chapter 6 , there is a need for the development and validation of a single tool to assess and grade the risk of bias of both trial- and model-based economic evaluations in health care. Such a 'risk of bias assessment tool' could make a substantial contribution to the field of health economics. It would assist end-users of economic evaluations to navigate the vast body of literature, and help producers of such studies to establish a clearer standard, potentially encouraging higher quality and greater rigor. ${ }^{39}$ 


\subsection{What are cost-effective treatments and strategies in epilepsy care?}

The review described in chapter 7, which provides an overview of all published economic evaluations within epilepsy, highlighted potential knowledge gaps regarding economic evidence in epilepsy. The majority of the studies focused on the cost-effectiveness of antiepileptic drugs $(71 \%)$, whereas the cost-effectiveness of other treatments, such as epilepsy surgery, the ketogenic diet, deep brain stimulations or self-management was studied significantly less often. Furthermore, this review stressed the need for a more standardized approach to conducting economic evaluations within the field of epilepsy.

Two of these less investigated treatments, namely the ketogenic diet and selfmanagement, were examined in chapters 8, 9 and 10. The first study (chapter 8) compared the ketogenic diet with care as usual in children and adolescents with intractable epilepsy. Although the ketogenic group showed more clinical improvements (not significantly after 16 months) there was no difference in quality of life between the two groups. Hence, at a maximum WTP of $€ 50,000$ per extra QALY, there was only a $25 \%$ chance that the KD would be cost-effective. However, the KD may still be considered to be a potentially important treatment for children with intractable epilepsy in patients who respond, given the large number of drop-outs resulting in a retention rate of $58 \%$. In patients who were able to tolerate the KD, results were promising. Moreover, the KD showed significant short-term (4 months) improvements on clinical outcomes such as the number of responders defined as a seizure reduction of $50 \%$ or more. This can be substantiated in the present study by the (positive) clinical outcomes for patients maintaining the diet. Further research should focus on determining which patients are more likely to benefit and tolerate the KD in order to improve the (cost-) effective application of the diet.

The second study compared a multi-component intervention $(\mathrm{MCl})$, aimed at increasing self-management skills (i.e. increase self-efficacy and concordance), with care as usual in adult patients with epilepsy. This intervention consisted of 1) group sessions; 2) the Medication Event Monitoring System (MEMS; Aardex Ltd., Switzerland); 3) a smartphone application "Eppy" (Epilepsy Foundation, the Netherlands); and 4) an internet accessible patient database. The group sessions involved groups of 3-5 patients and family members and/or friends. This study found an ICER of $€ 54$ per point increase in ESES-score at 6 months, and $€ 1105$ per point increase in ESES score at 12 months' follow-up. Concerning adherence, the intervention resulted in an ICER of $€ 88$ per percentage of adherence increase at 6 months. When looking at the QALYs, an ICUR of $€ 8,272$ per QALY gain was found at 6 months' follow-up and an ICUR of $€ 15,144$ per QALY gained at 12 months follow-up. Considering a threshold of $€ 50,000$ per QALY gained, the probability that the $\mathrm{MCl}$ is cost-effective is estimated to be $74 \%$ and $76 \%$ at 6 and 12 months' follow-up, respectively. No statistically significant differences were found in outcome measures between groups. It has, however, been argued that decisions should be based only on the net benefits, irrespective of whether differences are statistically significant, and that rules of 
inference are arbitrary and entirely irrelevant to the decisions which clinical and economic evaluations claim to inform. ${ }^{40}$

As self-management is a broad term used to describe the patients' ability to selfmanage his or her disease, the effect of interventions aimed at increasing self-management skills, such as the $\mathrm{MCl}$ in this study, may have varying clinical manifestations. In addition, self-management programs vary in content and manner of delivering information, thus limiting comparability with other studies. In the ZMILE study (chapter 9\&10), the effectiveness of the $\mathrm{MCl}$ was assessed using several outcomes such as medication adherence, self-efficacy, and (proactive) coping. Consequently, the effect of the $\mathrm{MCl}$ may have been spread among the different outcome measures. A recently developed instrument, the Adult Epilepsy Self-Management Measurement Instrument (ESMMI), was designed to provide an overall effectiveness measure for self-management interventions. ${ }^{41}$ The ESMMI is a synthesis of 11 instruments, including the ESES ${ }^{42}$ and the QOLIE-31p. ${ }^{43}$ The use of such an instrument, specifically designed for an intervention such as the $\mathrm{MCl}$, would have been a great addition to the current study.

\section{Further elaboration on health technology assessment in epilepsy}

This thesis makes the case for HTA being a broad discipline covering, among others, preference studies, partial and full economic evaluations, and (clinical) effectiveness studies. Though considered to be part of an overall discipline, these different subdisciplines are often not synthesized into an overall health technology assessment. If one is willing to move towards patient-centered and efficient care, some hurdles need to be taken to actually achieve this goal. Certain topics for discussion emerge from this statement. For example, preference studies are an important way to achieve insight into preferences. More research is, however, necessary to examine ways to incorporate the results into clinical or policy decision-making. Hence it is important to elaborate on methodological approaches to synthesize preferences in the decision-making process and to determine to what extent this is currently being done (see below).

Furthermore, it is often argued (also in this thesis) that helping patients to help themselves (self-management) may be a promising way to improve efficient use of health care resources. Hence, this chapter will expand on whether there is a need for selfmanagement in epilepsy. The last topic I would like to address regarding patient-centered and efficient care, is the quality and quantity of economic evaluations. Decent policy decisions can only be made on the basis of high quality research. It is, therefore, important to examine the quality of studies and to be able to differentiate when making decisions. Furthermore, the emphasis on patient-centered care also entails looking at the quantity of research and, more specifically, the representation of several (vulnerable) groups in research. According to professional and ethical codes for socio-economic research in the information society (the RESPECT Code of Practice ${ }^{44}$ ), vulnerable groups have the right to 
be protected from (potentially) harmful clinical studies, but also have the right to be represented in clinical studies. When identifying research gaps, one should take the treatment of under-represented vulnerable groups into account. These groups should be appropriately treated in all aspects, from research design to reporting the findings to ensure they are not excluded. At the same time, they should not be over-researched to avoid participation becoming a burden for them. ${ }^{44}$

\subsection{Preferences in clinical decision-making}

A large part of this thesis focusses around preference studies. It may, however, be unclear how (patients' or physicians') preferences are used in decision-making. During the last few decades, there has been a move towards including patients in decision-making ${ }^{45-48}$ and growing acceptance that the trade-offs made by actual patients differ from those of clinical experts. ${ }^{49}$ In addition, patient preferences have been shown to have a direct impact on the outcome of an intervention via psychological factors or indirectly via patient adherence/compliance rates. ${ }^{50}$ One way of incorporating patients' preferences is to include these in the clinical decision-making during consultation. It has been argued that there is a need for more research into decision-support mechanisms to enable patients to become informed participants in treatment decisions. ${ }^{51}$ For example, this could be achieved using decision aids during clinical consultations. According to the International Patient Decision Aid Standards Collaboration (IPDAS), decision aids are tools designed to help people participate in decision-making about health care options. ${ }^{52}$ They emphasize that decision aids do not advise people to choose one option over another, nor are they meant to replace practitioner consultation. Rather, they prepare patients so that they can make informed, values-based decisions with their practitioner. A Cochrane review by Stacey et al. ${ }^{53}$ found that decision aids appeared to have a positive effect on patientpractitioner communication, satisfaction with the decision, decision-making process, and/or preparation for decision-making. Those exposed to a decision aid were either more satisfied, or there was no difference between the decision aid versus comparison interventions. ${ }^{53}$ Using a decision aid, it is possible to match patient's preferences to the treatment choices.

One such aid is an option grid, a piece of paper with questions and answers which is used to enable communication between patients and physicians when deciding what would be the most suitable treatment(s) or test(s). ${ }^{54}$ Option grids are relatively simple and have recently been developed for people with epilepsy and are currently being tested in clinical practice.

Another form of decision aid is a decision support system (DSS) which can be based on multi-criteria decision analysis (MCDA). In MCDA, patients' preferences regarding benefits, risk factors and other attributes of the treatment are weighed. This 'weight' represents the importance of the attribute for the decision.

When these weights are combined with evidence about the specific attributes, the available treatments can be ranked from most preferred to least preferred. ${ }^{55}$ The BWS and 
DCE methods described above fulfill these criteria and are often labeled as MCDA techniques. DSSs based on MCDAs have been developed and used in other diseases. For example, the web-based online decision support platform Annalisa ${ }^{\odot}$ has been used as a DSS for patients regarding prostate cancer screening, ${ }^{56}$ Down's syndrome screening, ${ }^{57}$ and contraceptive choices, ${ }^{58}$ This DSS used a simple expected value algorithm to calculate a score for each option by taking into account the individual's preferences for different criteria (as importance weights) and the evidence for the performance of each option on each criterion. ${ }^{56}$ Although a direct relation between the use of such a DSS and clinical outcomes is lacking, in all three studies, the tool was regarded as being user-friendly le.g. ease of use, usefulness, understandable) and valuable in supporting decision-making by both patients and clinicians.

\subsection{Preferences in resource allocation decisions}

The use of patients' preferences in resource use allocation is also important, but practical guidance on how to incorporate preferences in existing HTA processes is often unclear. ${ }^{59}$ However, it has been argued that individual patient preferences for treatments should be taken into account over the entire spectrum of medical care, including the development of new treatment. An example of a possible implication of this is the case of anti-psychotics for schizophrenic patients. ${ }^{60}$ The literature suggests that negative side-effects are responsible for poor adherence of schizophrenics to anti-psychotics. However, a small study, in which participants were asked to rank the importance of medication attributes and effects, showed that patients with schizophrenia actually valued how clearly they could think and interact socially more than concerns about side-effects. ${ }^{61}$ Given this information, reducing the side-effect may not be the most effective way to improve adherence. In epilepsy, similar examples can be found. For example, McAuley et al. (2010) compared practitioners' impressions of patients' concerns with those expressed by the patients themselves. ${ }^{62}$ Although there was an overlap, patients were concerned more about life issues, and practitioners about clinical issues. ${ }^{62}$ Most interestingly, patients selected memory as the second-most important concern; this was weighted much more heavy by them than by their practitioners. However, practitioners did not see the memory problem as a major concern. This study demonstrated that it is important to epilepsy patients that future treatments should address memory problems, regardless of seizure control.

In an attempt to overcome this problem, Bridges \& Jones ${ }^{63}$ have proposed a framework called patient-based HTA, aimed at changing processes to increase patient participation at all levels of HTA and to promote empowering patients who can make informed decisions. ${ }^{63}$ Implications of ignoring patients' preferences would cause HTA to be discarded due to irrelevance, or for patients to be non-compliant with any treatment that is seemingly forced upon them. Another method, as suggested by Brazier et al., ${ }^{50}$ is to make use of a patient-based cost-effectiveness rule consisting of a two-part decision process. In this approach, the first part consists of the identification of the most cost-effective therapy using mean general population values (i.e. the current rule), then relative to this, the 
identification of those treatments that are cheaper. ${ }^{50}$ If a patient prefers treatment $B$ instead of the more cost-effective treatment $A$, they should be free to choose it, given that their own well-being will be greater under treatment $B$ relative to treatment $A$ and the mean costs are lower.

Currently, many decision makers in healthcare systems are looking into the use of patients' preferences to support decision-making. For example, National Institute for Health and Care Excellence (NICE) has taken patients' preferences into account as one approach to establishing the new scheme of value-based pricing (e.g. by eliciting willingness to pay). Furthermore, the Institute for Quality and Efficiency in Health Care (IQWiG) recommends preference elicitation techniques to weight the multiple endpoints in assessment processes and in support of health technology decision-making. ${ }^{59}$ The Evidence and Value: Impact on Decision-Making (EVIDEM) Collaboration used preference elicitation methods to rank healthcare interventions for reimbursement decisions. For example, this study demonstrated that, all else being equal, an intervention for a severe disease was assumed to have greater overall value than an intervention for a mild disease. However, several criteria related to the context of the decision may play a significant role, such as health care system capacity and barriers, stakeholder pressures, and ethical principles of resource allocation. ${ }^{64}$

Despite the increased attention to patients' preferences, no European HTA body is currently using formal preference elicitation approaches for regulatory decision-making. ${ }^{59}$ This stresses the need for further elaboration on methodologies which can be used to integrate patients', physicians', and decision-makers' preferences in resource allocation decisions. However, how one should deal with results of preference studies in decisionmaking remains unclear.

\subsection{The need for self-management in epilepsy?}

During the past decade there appears to have to have been an increase in emphasis on self-management in health care. It is important to determine whether this increased emphasis is indeed justified by a need for self-management in epilepsy (e.g. by demonstrated (cost-)effectiveness). Recently, there have been calls to change the oftenused definition of health, defined by the World Health Organization as "Health is a state of complete physical, mental and social well-being and not merely the absence of disease or infirmity". ${ }^{65}$ The proposed definition should be more dynamic and focus on patients' ability to self-manage his/her disease: "Health as the ability to adapt and implement their own control, in light of the physical, emotional and social challenges of life" .66 The National Institute for Public Health and the Environment (RIVM) has forecast that, with the aging population and the increase in life-expectancy, there is an growing emphasis on the capability of chronically ill people to design their lives optimally both as far as healthcare and local policy are considered. ${ }^{67}$ The RIVM also reports that there are people who lack the options and skills they need to control their own lives, usually those with lower levels of education and frail elderly people. They may benefit from additional care and support. 
A meta-analysis of Chodosh et al. ${ }^{68}$ looked at self-management programs for arthritis, diabetes, and high blood pressure and found that, compared with usual care, selfmanagement programs improved glucose and blood pressure control in older adults with diabetes and high blood pressure. Self-management programs offered only minimal improvement of pain and physical function in older adults with arthritis [68]. Another review by Barlow et al. ${ }^{69}$ provided an overview of publications on self-management in chronic conditions and concluded that, in general, the literature suggested that selfmanagement interventions have a beneficial effect on the well-being of participants, particularly in the short-term. However, long-term effects have been found for selfmanagement interventions. For example, Lorig et al. (2001) examined a Chronic Disease Self-Management Program for patients with heart disease, lung disease, stroke, or arthritis and demonstrated that after 1 and 2 years of follow-up, outpatient visits and health distress were reduced and self-efficacy improved. ${ }^{70}$ Moreover, for chronically ill frail elderly patients, a 1 -year self-management program including physical activity was associated with higher levels of physical activity and overall health status. ${ }^{71}$

In epilepsy, in addition to the review described in chapter 7, the Managing Epilepsy Well (MEW) Network has shown the lack of evidence-based programs for epilepsy selfmanagement despite the fact that, for example, e-Tools are indicated as promising support within epilepsy care. ${ }^{72}$ Furthermore, the MEW states that self-management skills can help people with epilepsy to take control of their health and cope with their day-to-day challenges. The two studies included in the review in chapter 7 deal with completely different interventions and have contradictory results. The study by Plumpton et al. ${ }^{73}$ was both less costly and more effective, whereas the study by Noble et al. ${ }^{74}$ was less effective and more costly. The study described in chapters $9 \& 10$ (ZMILE-study) did not find significant effects; however, economic evidence demonstrated that the $\mathrm{MCl}$ intervention had quite a high probability of being cost-effective. In addition, in their study, Oberié et al. concluded that medication adherence interventions (one of the components of the $\mathrm{MCl}$ described in chapter $9 \& 10$ ) can be cost-effective. Furthermore, they recommend that proven-effective adherence programs should be subjected to comprehensive economic evaluations. $^{75}$

Overall, self-management is promising and has gained attention over the last decade. So yes, there is a need for self-management in epilepsy. However, the large variation between interventions makes them difficult to compare and to derive (pooled) effectiveness estimates. Future research should aim to identify self-management program characteristics that predict successful outcomes. Furthermore, as stated by Barlow et al., ${ }^{69}$ greater use of peer education may offer a way forward, that not only builds on and values the experiences of people with chronic conditions, but may also prove to be cost-effective. Lastly, further research should try to show whether reinforcements can achieve sustained improvements and what time intervals are most cost-effective for such reinforcements. ${ }^{76}$ 


\subsection{The quantity and quality of economic evaluations in epilepsy}

In order to be able to identify possible knowledge or research gaps, it is important to identify the number, and corresponding quality, of economic evaluations within a specific field. The incorporation of economic evaluations in resource allocation decisions is increasingly becoming embedded in national guidelines. For example, the use of economic evaluations is required by the National Institute for Health and Clinical Excellence (NICE) in the UK ${ }^{77}$ and used by the National Health Care Institute in the Netherlands for health technology assessment. ${ }^{78}$ The healthcare budget is used to promote overall health and to improve productivity and efficiency of healthcare by investments in healthcare facilities (e.g. technologies, programs and interventions). ${ }^{79}$ As the budget is insufficient to cover all healthcare expenses, policy makers need to create the most costeffective and health-producing distribution from the limited budget. Evidence-based analyses of interventions and treatments are important for adequate decision-making. This stresses the need for evidence-based economic evaluations within the healthcare sector.

As shown in chapter 7 , there is room for improvement when it comes to the conduct of economic evaluations in the field of epilepsy in terms of quantity, quality, and comparability. This may be due to a lack of awareness of economic evaluations among clinicians conducting clinical trials or a lack of knowledge of economic evaluations. For example, despite the long-recognized need for QALYs in priority-setting and decisionmaking and the growing evidence that psychosocial and poor mental health have the greatest impact on quality of life, ${ }^{80,81}$ many studies focus primarily on seizure frequency. As stated by Perucca et al. (2009), "the lack of investigations towards other clinical factors can be ascribed to the historical belief that seizure frequency is the major determinant of quality of life". ${ }^{82}$ Hence, a reference case may provide general guidance in conducting economic evaluations within epilepsy and may increase standardization and comparability between studies. Economic evaluations can vary in a variety of aspects, all of which can affect the quality of the evaluation. Hence, to structure the most important aspects, the Centre for Reviews and Dissemination (CRD) has identified several quality indicators for the appraisal of economic evaluations ${ }^{83}$ : methods of deriving the effectiveness data; measurement of resource data; valuation of the resource data; measurement and valuation of health benefits (utilities); method of synthesizing the costs and effects; and the analysis of uncertainty. These quality indicators may provide a great basis for developing a reference case specific for epilepsy.

Furthermore, as also shown in chapter 7 , some groups were underrepresented in the identified studies: children, the elderly or patients with cognitive deficits which may give rise to problems such as important information deficits and a potential lack of applicability of evidence generated from clinical trials to vulnerable populations. ${ }^{84}$

Next, often overlooked but no less important, is the reporting of an economic evaluation. After all, a publication describing a high-quality study could receive a low quality appraisal because vital information is not reported adequately. In 1996, Gold et al. ${ }^{85}$ developed a checklist, called the U.S. Panel checklist, for reporting clinical practice guidelines (CPGs). This list consists of 38 questions specifically related to reporting cost- 
effectiveness analyses and, although it can be used for both trial- and model-based EE, it is more applicable to trial-based EE. An ISPOR taskforce has developed the Consolidated Health Economic Evaluation Reporting Standards" (CHEERS), which gives recommendations for optimizing the reporting of health economic evaluations. ${ }^{86}$ These recommendations were developed using a two-round Delphi panel; they include 24 items and accompanying recommendations, some specifically for trial-based and model-based economic evaluations. There is, however, currently no reporting guidance available specifically tailored to systematic reviews of economic evaluations.

CPGs have a large impact on many health care decisions for patients, not only those involving reimbursement. Due to the constant and worldwide tension between clinical opportunities and what is financially feasible, there is a need for a critical approach to healthcare expenditure and the place of economic evidence in CPGs. Though recommended, economic evaluations (EEs) are not routinely incorporated in clinical practice guidelines. ${ }^{87}$ Studies investigating the uptake of HTA-studies in clinical practice found that this is generally limited, especially at the local level. ${ }^{88,89}$ Until 2014 , economic evidence was not incorporated into the Dutch clinical practice guidelines, which were based on scientific research (clinical trials) supplemented by the expertise of health care professionals and patients. As part of the project "How to Prepare a Systematic Review of Economic Evaluations for Informing Evidence-based Healthcare Decisions", (part III is described in chapter 6), an overview of all knowledge and practical information needed to systematically incorporate EE in clinical practice guidelines was provided for CPG developers. This framework included a flowchart containing the different steps of the review process, guidance on the development and selection of search strategies, guidance on selection of different bibliographic databases, information on how to select and use checklists to appraise the methodological quality of the studies, information on how to handle transferability and generalizability issues, guidance on data extraction and data syntheses. It is currently being used to identify new articles systematically, to process such articles, and update and expand the Dutch CPG for epilepsy on a yearly basis to include economic evidence. This standardized approach is expected to facilitate the incorporation of EEs in (other) new and existing clinical practice guidelines. However, despite methodological frameworks, including economic evidence in CPG may not be an easy process. For example, with a restricted budget, the use of economic evaluations is always associated with bad news for some parties. ${ }^{88}$ Moreover, in 2012 when NICE revised the CPG for epilepsy in the UK, the guidelines came under fire by several experts who said that they did not reflect clinical experience and focused too much on drug costeffectiveness. $^{90}$

Although chapter 6 emphasizes the importance of full economic evaluations, partial economic evaluations may be useful for CPG development. While a partial economic evaluation does not directly provide answers to efficiency issues, it is important in offering insights into the (societal) burden of a specific condition. In turn, this can be used for priority setting (e.g. prioritizing research agendas), or to identify factors responsible for relatively high costs which could be subjected to efficiency research. Partial economic evaluation is a 
broadly used term covering a wide range of studies, such as cost of illness studies and budget impact analyses. Partial economic evaluations can be useful for policy-makers in a more indirect manner. Especially in the case of CPG development, if little is known about a particular treatment, partial economic evaluations may provide useful results.

Transparency and the involvement of a broad range of stakeholders (e.g. clinicians but also health economists, insurance companies and patient representatives) are, therefore, important to build sufficient support.

\subsection{The role of education in proper and widely applied health technology assessment}

So far this thesis has highlighted shortcomings and topics for discussion in the field of HTA, mainly with regard to more advanced methodological aspects Many of the methodologies used in HTA research, e.g. the conduct of trial- or model-based economic evaluations and preference-based studies such as DCE or TTO, are well-founded and experts have reached a consensus over the past decades.

Hence, there may be an important role for increasing awareness and knowledge to achieve wide spread use and uniformity in the conduct of HTA in health care. For example, in a report of the Netherlands Federation of University Medical Centers in 2014 it was cited that "most clinicians acknowledge that only half of the procedures they perform are based on scientific evidence, let alone cost-effectiveness". ${ }^{91}$ Moreover, the Dutch Federation of Medical Specialists developed a quality cycle for the successful clinical and cost-effectiveness evaluation of care in which education plays an important role. ${ }^{92}$ This report also reflects on the view of the National Health Care Institute and the Dutch Hospital Association who want to encourage medical professionals to increase the involvement of cost-effectiveness in care delivery. This trend is also observed in other countries. For example, most influential medical groups in the USA are recommending that doctors weigh the costs, not just the effectiveness of treatments, as they make decisions about patient care. ${ }^{93}$ Until a few years ago, HTA, and more specifically economic evaluation, was not embedded in medical education. However, a taskforce arising from the initiative Modernisering Medische Vervolgopleidingen (Modernization of Continued Medical Education), part of the Dutch Federation of Medical Specialists, has recently developed a more detailed competence-based approach for continued medical education in which efficiency research is one of the key components. ${ }^{94}$ Each competency is subdivided into three layers: 1) basic knowledge, which is relevant for all students; 2) advanced knowledge, which is dedicated to senior students who are looking to gain more in-depth knowledge; and 3) knowledge for talents, which is dedicated to students who want to gain even more in-depth knowledge, possibly in a sub-discipline. For every medical student, an individually tailored education plan, agreed by both teacher and student, is compiled at the start of their education. The aim of the taskforce is to provide practical guidance on several competencies instead of solely theoretical descriptions, and to encourage medical teachers to use this competence-based approach nationwide. 
Embedding economic evaluations within medical education is a great way to increase the awareness and possibly the use of economic evidence in health care. Integrating health economics into medical education, for example, encourages an appreciation of why economic factors influence clinical decision-making. ${ }^{95}$ It has been argued that integrating health economics into medical education enables doctors to assess the economic value of medical programs, supporting problem-solving and contributing to health policy analyses. ${ }^{96}$ In addition, cost considerations by clinicians has been shown to reduce practice variation, ineffectiveness, and wasteful care while improving outcomes and encouraging the use of proven therapies. ${ }^{97}$

Although the important role of economic evidence in the education of medical specialists has been recommended in the past decade, this is still only a part of HTA. There may be room for a broader focus on HTA within medical education which would also include preference studies.

\section{Concluding remarks \& recommendations}

The aim of this thesis is two-fold; the first part is devoted to methodological aspects and preference-based studies in the health technology assessment of epilepsy; and the second part to economic evaluations within epilepsy. In the first part, great emphasis was placed on preference-based studies ranging from a discrete choice experiment in patients with epilepsy, to a time-trade off study in the general population and a review to examine the use of best-worst scaling in health care. The second part discussed the risk of bias assessment for economic evaluations and the results of two economic evaluations. In addition, this part highlighted areas of potential research gaps within the field of epilepsy, especially in vulnerable groups such as patients with cognitive deficits, elderly patients, and the treatment of (non)convulsive status epilepticus in both children and adults.

The distinction between preference-based studies and economic evaluation was made in both parts to provide structure. However, by presenting these parts as distinct topics, I indirectly contribute to the lack of synthesis of the two disciplines in (policy or clinical) decision-making. Returning to the title of this thesis, namely "Health technology assessment in epilepsy; moving towards patient-centered and efficient care": this may seem contradictory. But although they are presented in this thesis as strictly two scientifically distinct disciplines, integration on a macro-economic level (e.g. resource allocation decision) or micro-economic level (e.g. clinical consultations) is necessary in order to meet the needs of patients, clinicians, and policy makers and to achieve an efficient use of resources. Shared-decision making, involving all relevant stakeholders, constitutes better tailored care and may enhance concordance (i.e. consensual agreement about treatment established between patient and practitioner), not only in patients with epilepsy, but in medicine as a whole. In accordance with Deber (1994), historical views on the relationship between patients and physicians, e.g. that the physician's role was to act in the best interests of the patient and to direct care and make decisions about treatment on the 
patient's behalf, are no longer sufficient; respect for autonomy of the patient is important, necessitating patient participation. ${ }^{98}$

Several recommendations have emerged from this thesis:

- The first is to obtain greater insight into measurement of quality of life in epilepsy as the EQ-5D-5L was shown to lack responsiveness. This may stress the need for the development of a condition-specific preference-based measure which can then be used to calculate condition-specific QALYs as a supplement to economic evaluations. The development of such condition-specific preference-based quality of instruments may ensure that the benefits of health-care interventions are adequately reflected in QALY estimates for economic evaluations. Although the use of the EQ-5D-5L is recommended by the National Health Care Institute, HTA researchers should be careful when using the EQ-5D-5L in epilepsy research. As long as there is no clear answer, it is probably best to use both generic and disease-specific instruments.

- The second recommendation is to investigate the use and (cost-)effectiveness of decision aids in clinical decision-making as a way of incorporating patients' preferences in clinical decision-making.

- Third, related to the second recommendation, further research should focus on methodologies which can be used to integrate patients', physicians', and decisionmakers' preferences in resource allocation decisions. There is a need for consensus on how to deal with preferences in resource allocation and clinical decision-making.

- Fourth, there is a need for the development and validation of a single tool to assess and grade the risk of bias of both trial-and model-based economic evaluations in health care. Such a 'risk of bias assessment tool' could make a substantial contribution to the field of health economics. In addition, there is currently no reporting guidance available specifically tailored to systematic reviews of economic evaluations. Such guidelines might increase transparency and comparability between reviews and make it easier for policy-makers or subsidizing parties to identify research gaps.

- Fifth, there is a need for a more standardized approach to conducting economic evaluations within the field of epilepsy. There are (current) guidelines on how to conduct economic evaluations in health care; however, an epilepsy-specific guideline could focus, for example, on preferred clinical outcome measures (i.e. 50\% seizure reduction or proportion of seizure-free patients), preferred utility instruments (i.e. a more extensive instrument than solely the EQ-5D-5L), and preferred or basic model structures for model-based economic evaluations within epilepsy. A reference-case might provide a viable and feasible solution.

- Sixth, there are potential gaps in knowledge regarding economic evidence in epilepsy. The majority of the studies currently focus on the cost-effectiveness of antiepileptic drugs, whereas the cost-effectiveness of other treatments, such as epilepsy surgery, the ketogenic diet or self-management were studied significantly less often. In addition, studies on vulnerable groups are lacking. 
- Seventh, future research should focus on determining which patients are more likely to benefit and tolerate the ketogenic diet to increase a (cost-) effective application of the diet.

- Eighth further research should attempt to show whether reinforcements in (educational) self-management interventions can achieve sustained improvements, and which time intervals are most cost-effective for such reinforcements.

- Ninth, it is important to include HTA in medical education. Currently, more emphasis is placed on economic evaluations, a welcome development, but the scope could be widened by increasing emphasis on other aspects of HTA, such as preference studies.

- Lastly, given their increased importance, it is recommended that an economic evaluation is always performed when conducting a clinical trial. These (piggy-backed) trial-based economic evaluations should be conducted taking into account the latest guidelines provided by the National Health Care Institute. 


\section{References}

1. Wijnen B, et al. Eliciting patients' preferences for epilepsy diagnostics: A discrete choice experiment. Epilepsy Behav 2014;31:102-109.

2. de Kinderen RJ, et al., From clinically relevant outcome measures to quality of life in epilepsy: A time tradeoff study. Epilepsy Res 2016;125:24-31.

3. Treadwell JR, Lenert LA. Health values and prospect theory. Med Decis Making 1999;19(3):344-52.

4. Wilson TD, Gilbert DT. Affective forecasting: Knowing what to want. current directions in psychological science $2005 ; 14(3): 131-134$.

5. Neumann PJ, Goldie SJ, Weinstein MC. Preference-based measures in economic evaluation in health care. Annu Rev Public Health 2000;21(1):587-611.

6. Versteegh M, et al. Dutch Tariff for the Five-Level Version of EQ-5D. Value in Health, 2016. 19(4): p. 343352.

7. Brazier J, Roberts J, Deverill M. The estimation of a preference-based measure of health from the SF-36. J Health Econ 2002;21(2):271-292.

8. McCabe $\mathrm{C}$, et al. Health state values for the HUI 2 descriptive system: results from a UK survey. Health Econ $2005 ; 14(3): 231-244$.

9. Weinstein $M$, et al. Cost-effectiveness in health and medicine. New York: Oxford University 1996. 55.

10. (TLV), T.D.a.P.B.A., General Guidelines for Economic Evaluations from the Pharmaceutical Benefits Board (LFNAR2003:2). 2003: Stockholm.

11. Versteegh MM, Brouwer WB. Patient and general public preferences for health states: A call to reconsider current guidelines. Soc Sci Med 2016;165:66-74.

12. Coons SJ, et al. A comparative review of generic quality-of-life instruments. Pharmacoeconomics 2000; $17(1): 13-35$.

13. Von Neumann J, Morgenstern O. Theory of games and economic behavior. 2007: Princeton university press.

14. Torrance GW. Utility Measurement in Healthcare. Pharmacoeconomics 2006;24(1 1):1069-1078.

15. Versteegh MM, et al. Condition-Specific Preference-Based Measures: Benefit or Burden? Value Health 2012;15(3):504-513.

16. Brazier J, Tsuchiya A. Preference-based condition-specific measures of health: what happens to cross programme comparability? Health Econ 2010;19(2):125-129.

17. Brazier JE, et al. Developing and testing methods for deriving preference-based measures of health from condition-specific measures (and other patient-based measures of outcome). Health Technol Assess 2012; 16(32):1-114.

18. Richardson J, et al. Increasing the sensitivity of the AQoL inventory for evaluation of interventions affecting mental health. Research paper 2011.61.

19. Al-Janabi H, Flynn TN, Coast J. Development of a self-report measure of capability wellbeing for adults: the ICECAP-A. Qual Life Res 2012;21(1):167-176.

20. Cheung KL, et al. Using Best-Worst Scaling to Investigate Preferences in Health Care. Pharmacoeconomics, 2016.

21. Clark MD, et al. Discrete choice experiments in health economics: a review of the literature. Pharmacoeconomics 2014;32(9):883-902.

22. Potoglou $D$, et al. Best-worst scaling vs. discrete choice experiments: an empirical comparison using social care data. Soc Sci Med 201 1;72(10):1717-27.

23. Gallego G, et al. Should I stay or should I go? Exploring the job preferences of allied health professionals working with people with disability in rural Australia. Hum Resources Health 2015;13(1):53.

24. Lancsar $E$, et al. Best worst discrete choice experiments in health: methods and an application. Soc Sci Med, 2013;76(1):74-82.

25. Bridges JF, et al. Conjoint analysis applications in health-a checklist: a report of the ISPOR Good Research Practices for Conjoint Analysis Task Force. Value Health 2011 ; 14(4):403-413.

26. Hauber $A B$, et al. Statistical methods for the analysis of discrete choice experiments: A report of the ISPOR conjoint analysis good research practices task force. Value Health 2016.

27. Johnson FR, et al. Constructing experimental designs for discrete-choice experiments: report of the ISPOR conjoint analysis experimental design good research practices task force. Value Health 2013; 16(1):3-13. 
28. Mühlbacher AC, et al. Experimental measurement of preferences in health and healthcare using best-worst scaling: an overview. Health Econ Rev 2016;6:2.

29. Muhlbacher AC, et al.. Experimental measurement of preferences in health care using best-worst scaling (BWS): theoretical and statistical issues. Health Econ Rev 2016;6(1):5.

30. Nederlandse vereniging voor epilepsie. Richtlijnen Epilepsie. 2016 28-10-2016]; Available from: http://epilepsie.neurologie.nl/cmssite/index.php?pageid=2.

31. Drummond MF, et al. Methods for the economic evaluation of health care programmes. 2015: Oxford university press.

32. Evers SM, Hiligsmann M, Adarkwah CC. Risk of bias in trial-based economic evaluations: identification of sources and bias-reducing strategies. Psychol Health 2015;30(1):52-71.

33. Evers $\mathrm{S}$, et al. Criteria list for assessment of methodological quality of economic evaluations: Consensus on Health Economic Criteria. Int J Technol Assess Health Care 2005;21 (2):240-5.

34. Odnoletkova I, et al. Cost-effectiveness of therapeutic education to prevent the development and progression of type 2 diabetes: Systematic review. J Diabetes Metab 2014. 2014.

35. Drummond, M.F. and T.O. Jefferson, Guidelines for authors and peer reviewers of economic submissions to the BMJ. BMJ 1996;313(7052):275-83.

36. Philips $Z$, et al. Good practice guidelines for decision-analytic modelling in health technology assessment: a review and consolidation of quality assessment. Pharmacoeconomics 2006;24(4):355-71.

37. Jaime Caro J, et al. Questionnaire to assess relevance and credibility of modeling studies for informing health care decision making: an ISPOR-AMCP-NPC Good Practice Task Force report. Value Health 2014; 17(2):174-82.

38. Welte $R$, et al. A decision chart for assessing and improving the transferability of economic evaluation results between countries. Pharmacoeconomics 2004;22(13):857-76.

39. Walker DG, et al. AHRQ Methods for Effective Health Care, in Best Practices for Conducting Economic Evaluations in Health Care: A Systematic Review of Quality Assessment Tools. 2012, Agency for Healthcare Research and Quality (US): Rockville (MD).

40. Claxton K. The irrelevance of inference: a decision-making approach to the stochastic evaluation of health care technologies. J Health Econ 1999; 18(3):341-64.

41. Escoffery C, et al. Development of the Adult Epilepsy Self-Management Measurement Instrument (AESMMI). Epilepsy Behav 2015;50:172-83.

42. Dilorio C, Yeager K. The epilepsy self-efficacy scale. Teoksessa: Strickland OL \& Dilorio C (toim.) Measurement of nursing outcomes: Self care and coping, New York, 2003:40-51.

43. Cramer JA, Van Hammée G, Group NS, Maintenance of improvement in health-related quality of life during long-term treatment with levetiracetam. Epilepsy Behav 2003;4(2):1 18-23.

44. Professional and ethical codes for socio-economic research in the information society. The RESPECT Code of Practice. 2004 30-11-2016]; Available from: http://www.respectproject.org/code/index.php?id=.

45. Elwyn G, et al. Shared decision making and the concept of equipoise: the competences of involving patients in healthcare choices. Br J Gen Pract 2000;50(460):892-9.

46. Elwyn G, Edwards A,Kinnersley P. Shared decision-making in primary care: the neglected second half of the consultation. Br J Gen Pract 1999;49(443):477-82.

47. Say $R$, Murtagh $M$, Thomson R. Patients' preference for involvement in medical decision making: a narrative review. PatientEduc Couns 2006;60(2):102-14.

48. Mühlbacher AC, et al. Patient-Focused Benefit-Risk Analysis to Inform Regulatory Decisions: The European Union Perspective. Value Health 2016;19(6):734-40.

49. Mühlbacher AC, Juhnke C. Patient preferences versus physicians' judgement: does it make a difference in healthcare decision making? Appl Health Econ Health Policy 2013;1 1(3):163-80.

50. Brazier JE, Dixon S, Ratcliffe J. The role of patient preferences in cost-effectiveness analysis: a conflict of values? Pharmacoeconomics 2009;27(9):705-12.

51. Coulter A. Partnerships with Patients: The Pros and Cons of Shared Clinical Decision-Making. Journal of Health Services Research 1997;2(2):1 12-21.

52. International Patient Decision Aid Standards Collaboration. What are patient decision aids? 2016 28-10$2016] ;$ Available from: http://www.ipdas.ohri.ca/what.html.

53. Stacey $D$, et al. Decision aids for people facing health treatment or screening decisions. Cochrane Database Syst Rev 2011 (10):Cd001431.

54. Epilepsiefonds Nederland, Jaarverslag 2015. 2015, Stichting Epilepsiefonds: Utrecht. 
55. Dowie J, et al. Towards generic online multicriteria decision support in patient-centred health care. Health Expect $2015 ; 18(5): 689-702$.

56. Cunich $M$, et al. Integrating evidence and individual preferences using a web-based multi-criteria decision analytic tool: an application to prostate cancer screening. Patient $2011 ; 4(3): 153-62$.

57. Erenbourg A, et al. Decision support in Down's syndrome screening using multi-criteria decision analysis: a pilot study. Epidemiology Biostatistics Public Health 2013. 10(3).

58. French RS, et al. The development of a multi-criteria decision analysis aid to help with contraceptive choices: My Contraception Tool. J Fam Plann Reprod Health Care 2014;40(2):96-101.

59. Mühlbacher AC, Kaczynski A. Making Good Decisions in Healthcare with Multi-Criteria Decision Analysis: The Use, Current Research and Future Development of MCDA. Applied Health Economics and Health Policy 2016;14(1):29-40.

60. Buttorff C. What Should Be the Role of Patient Preferences in Making Health Care Resource Allocation Decisions?, in ISPOR 12th Annual European Congress. 2009, International Society for Pharmacoeconomics and Outcomes Research: Paris.

61. Kinter ET, et al. Identifying patient-relevant endpoints among individuals with schizophrenia: an application of patient-centered health technology assessment. Int J Technol Assess Health Care 2009;25(1):35-4 1.

62. McAuley JW, et al. Comparing patients' and practitioners' views on epilepsy concerns: A call to address memory concerns. Epilepsy Behav 2010;19(4):580-3.

63. Bridges JF, Jones C. Patient-based health technology assessment: a vision of the future. Int J Technol Assess Health Care 2007;23(01):30-5.

64. Goetghebeur MM, et al. Bridging health technology assessment (HTA) and efficient health care decision making with multicriteria decision analysis (MCDA): applying the EVIDEM framework to medicines appraisal. Med Decis Making 2012;32(2):376-88.

65. World Health Organization. WHO definition of Health. 2016 28-10-2016]; Available from: http://www.who.int/about/definition/en/print.html.

66. Huber $M$, et al. How should we define health? BMJ 2011.343.

67. National Institute for Public Health and the Environment RIVM forecasting study: a healthier Netherlands with more people living with a chronic disease. 2014 28-10-2016]; Available from: http://www.rivm.nl/en/Documents_and_publications/Common_and_Present/Newsmessages/2014/RI VM_forecasting_study_a_healthier_Netherlands_with_more_people_living_with_a_chronic_disease.

68. Chodosh J, et al. Meta-analysis: Chronic disease self-management programs for older adults. Ann Int Med 2005; 143(6):427-38.

69. Barlow J, et al. Self-management approaches for people with chronic conditions: a review. Patient Educ Couns 2002;48(2):177-87.

70. Lorig KR, et al. Chronic disease self-management program: 2-year health status and health care utilization outcomes. Med Care 2001;39(11):1217-23.

71. Leveille SG, et al. Preventing Disability and Managing Chronic Illness in Frail Older Adults: A Randomized Trial of a Community-Based Partnership with Primary Care. Journal of the American Geriatrics Society, 1998;46(10):1191-8.

72. Shegog R, et al. Managing Epilepsy Well: Emerging e-Tools for epilepsy self-management. Epilepsy Behav 2013;29(1):133-40.

73. Plumpton $\mathrm{CO}$, et al. Economic evaluation of a behavior-modifying intervention to enhance antiepileptic drug adherence. Epilepsy Behav 2015;45:180-6.

74. Noble AJ, et al. Clinical- and Cost-Effectiveness of a Nurse Led Self-Management Intervention to Reduce Emergency Visits by People with Epilepsy. PLoS One 2014;9(3):e90789.

75. Oberié EJM, et al. Cost effectiveness of medication adherence-enhancing interventions: $A$ systematic review of trial-based economic evaluations. Pharmacoeconomics 2013;31(12):1155-68.

76. Goudswaard AN, et al. Long-term effects of self-management education for patients with Type 2 diabetes taking maximal oral hypoglycaemic therapy: a randomized trial in primary care. Diabet Med 2004; $21(5): 491-6$.

77. Williams I, et al. The use of economic evaluations in NHS decision-making: a review and empirical investigation. Health Technol Assess 2008; 12(7):iii, ix-x, 1-175.

78. Zorginstituut Nederland. Economische evaluatie. 2016 15-11-2016]; Available from: https://www.zorginstituutnederland.nl/pakket/werkwijze+pakketbeheer/beoordeling+geneesmiddelen/ economische+evaluatie. 
79. Annemans L. Gezondheidseconomie voor niet-economen. Een inleiding tot de begrippen, methoden en valkuilen van de gezondheidseconomische evaluatie. 2007: Academia Press.

80. Perrine K, et al. The relationship of neuropsychological functioning to quality of life in epilepsy. Arch Neurol 1995;52(10):997-1003.

81. Boylan LS, et al. Depression but not seizure frequency predicts quality of life in treatment-resistant epilepsy. Neurology 2004;62(2):258-61.

82. Perucca P, Gilliam FG, Schmitz B. Epilepsy treatment as a predeterminant of psychosocial ill health. Epilepsy Behav 2009; 15 Suppl 1:S46-50.

83. Tacconelli E. Systematic reviews: CRD's guidance for undertaking reviews in health care. The Lancet Infect Dis 10(4):226.

84. Rogers W, Lange MM. Rethinking the Vulnerability of Minority Populations in Research. Am J Public Health 2013;103(12):2141-6

85. Gold M. Panel on cost-effectiveness in health and medicine. Medical Care 1996;34(12):DS197-DS199.

86. Husereau D, et al. Consolidated Health Economic Evaluation Reporting Standards (CHEERS)--explanation and elaboration: a report of the ISPOR Health Economic Evaluation Publication Guidelines Good Reporting Practices Task Force. Value Health 2013;16(2):231-50.

87. van Mastrigt GA, Hiligsmann M, Arts JJ, Broos PH, Kleijnen J, Evers SM, Majoie MH. How to Prepare a Systematic Review of Economic Evaluations for Informing Evidence-based Healthcare Decisions: A five-step approach (part 1 of 3). Expert Rev Pharmacoecon Outcomes Res. 2016;16(6):689-704.

88. Drummond M. Economic Evaluation in Health Care: Is It Really Useful or Are We Just Kidding Ourselves? Australian Economic Review 2004;37(1):3-11.

89. Briggs A, A View from the Bridge: Health Economic Evaluation - A Value-Based Framework? Health Economics 2016;25(12):1499-502.

90. Holmes D. NICE epilepsy guidance may be detrimental to patient care. Lancet 2012;379(9814):399.

91. Task force of the Netherlands Federation of University Medical Centres, Zichtbaar zinnige en zuinige zorg. 2014.

92. Stuurgroep Zorgevaluatie, Adviesrapport Zorgevaluatie Van project naar proces. 2016.

93. Pollack A. Cost of Treatment May Influence Doctors, in The New York Times. 2014.

94. Denktank Modernisering Medische Vervolgopleidingen, Van CanMEDS naar CanBetter; Hoe leid je aios op in algemene competenties? 2016.

95. Evans DB. Medical education and economics. Med Educ 1989;23(1):48-54

96. Da'ar OB, Al Shehri AM. Towards integration of health economics into medical education and clinical practice in Saudi Arabia. Medical Teacher 2015;37(sup 1):S56-S60.

97. Scott RD, Solomon SL, McGowan JE. Applying economic principles to health care. Emerging Infectious Diseases $2001 ; 7(2): 282-5$.

98. Deber RB. Physicians in health care management: 7. The patient-physician partnership: changing roles and the desire for information. CMAJ: Canadian Medical Association Journal 1994;151(2):171-6. 


\section{Summary}
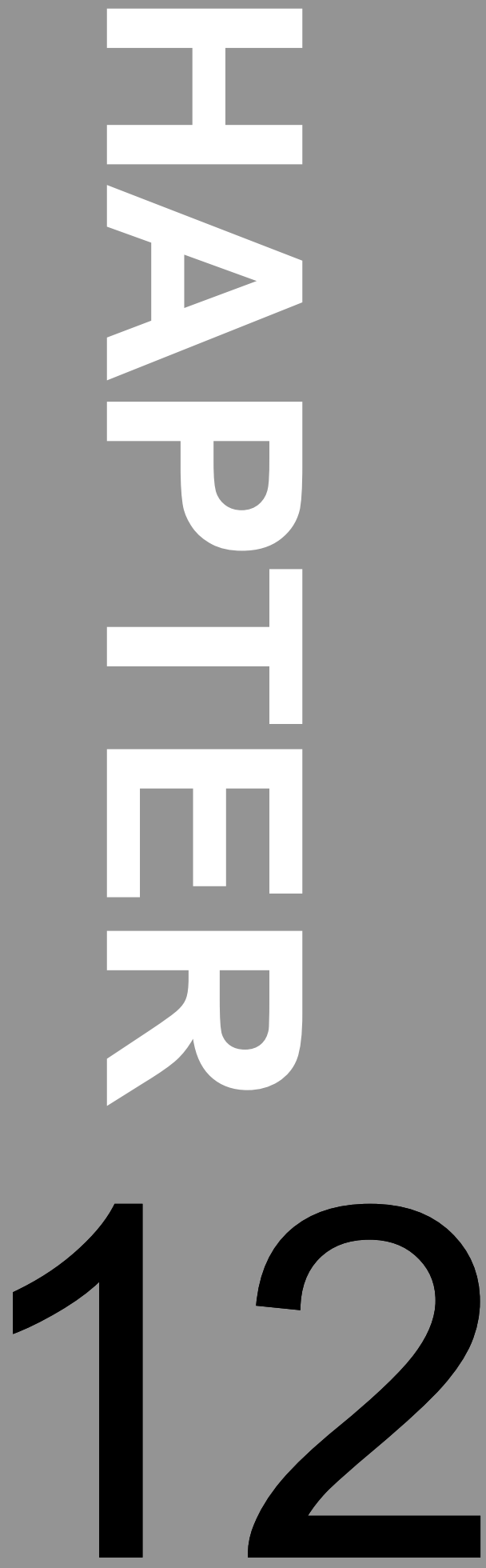



\section{Summary}

Health technology assessment (HTA) is a multidisciplinary field in which, among others, costs, (cost-)effectiveness and preferences play an important role. Given the rising health care expenditures the past decades in almost all developed countries, economic evaluations and the accompanied cost-effectiveness criterion have become an important part of healthcare policy decisions. In addition, the increased awareness of patient autonomy, growing acceptance that the trade-offs made by actual patients differ from those of clinical experts, and the changing relation between patient and clinician necessitate the need for a more patient-centered healthcare system. This thesis reports on methodological aspects and preference-based studies in the health technology assessment of epilepsy; and on the availability and quality of economic evaluations within epilepsy. Lastly, this thesis reports on two economic evaluations: one study evaluating the costeffectiveness of the ketogenic diet in children with intractable epilepsy, and one study evaluating the cost-effectiveness of a multi component self-management intervention for adults with epilepsy.

Chapter 1 provides background knowledge regarding epilepsy and highlights relevant concepts within HTA. Epilepsy is a neurological disorder, characterized by recurrent unprovoked seizures. About $30 \%$ of the patients with epilepsy are not seizure free while being on anti-epileptic drugs (AEDs). These patients, with uncontrolled epileptic seizures and in which two or more AEDs have failed, are commonly referred to have refractory or intractable epilepsy. Epilepsy has a large impact on patients' quality of life, their surrounding and society as a whole.

Health technology assessment is a broad multidisciplinary field which examines the social, economic, organizational and ethical issues of a health intervention or health technology. HTA aims to provide policymakers and clinicians evidence-based choices in health services and patient care. Two central HTA topics in this thesis are preference elicitation and economic evaluations. Preference elicitation studies try to prioritize and identify preferences from pre-specified stakeholders to inform policy or clinical decisions. Preferences in healthcare are typically elicited by using stated preference methods, such as a discrete choice experiment (DCE), best-worst scaling (BWS), or time trade-off (TTO) exercises. In economic evaluations one compares both the costs and effects of two (or more) alternative treatments or interventions in a systematic manner to calculate incremental cost-effectiveness ratios.

This chapter ends with an overview of the research questions which were used as a basis for this thesis.

Chapter 2 describes a DCE to identify to what extent important attributes of diagnostic procedures in epilepsy affect preferences for a procedure; 2) to determine the relative importance of these attributes; 3) to calculate overall utility scores for routine electroencephalography (EEG) and magnetoencephalography (MEG) recordings. 
Respondents were asked to make a series of binary choices between two (hypothetical) diagnostic procedures used in epilepsy, based on several attributes which varied in levels. It was demonstrated that MEG is not necessarily more patient-friendly than a routine EEG in primary diagnostics and, regarding additional diagnostics, patients had a strong preference for long-term 24h EEG over EEG after sleep deprivation.

Chapter 3 reports on the results of a time trade-off (TTO) study in which utility scores were derived for epilepsy health states. In this study health states, based on clinically important epilepsy attributes (e.g. seizure frequency, seizure severity, side-effects), were valued by a sample of the Dutch population $(\mathrm{N}=525)$ using the TTO method. This study provides a utility-function for transforming clinically relevant epilepsy outcome measures into utility estimates. In addition, it appeared that seizure severity had the largest impact on quality of life compared with seizure frequency and side-effects.

Chapter 4 describes a study in which the responsiveness of and correlation between the EQ-5D-5L and the QOLIE-31P in patients with epilepsy is examined. The EQ-5D-5L showed substantially high ceiling effects and rather small effect sizes (EFs) and standardized response means (SRMs) whereas the QOLIE-3 1 P did not show ceiling effects and also showed small to moderate EFs and SRMs. Mapping functions to regress QOLIE$31 \mathrm{P}$ values to $\mathrm{EQ}-5 \mathrm{D}-5 \mathrm{~L}$ values did not show an optimal fit with relatively low adjusted R2 values. The results presented in this study may emphasize the importance of the development of condition-specific preference-based instruments which can be used within the QALY framework and hence incorporated as an important supplement in economic evaluations.

Chapter 5 presents a systematic review of current practice and trends in the use of best-worst scaling (BWS) in health care. The review aimed to identify, review and critically appraise BWS in health care, and to identify trends over time in key aspects of BWS. A total of 62 BWS were identified of which 26 were BWS object case, 29 were BWS profile case, and 7 were BWS multi-profile case. About two- thirds of studies were performed in the last two years. Decreasing sample sizes and number of factors in BWS object case, as well as the use of less complicated analytic methods was observed in recent studies. The quality of BWS studies was generally acceptable according to the PREFS checklist. The use of BWS has drastically increased in health care, especially in the last two years.

Chapter 6 provides guidance on the conduct of a systematic review of economic evaluations (EEs) for informing evidence-based healthcare decisions. More specifically this paper explains the data extraction process, the risk of bias assessment and the transferability of EEs. Recommendations are based on criteria lists are based on the thoroughness of development, feasibility, overall quality, recommendations of leading organizations, and widespread use. 
Chapter 7 presents a systematic review of economic evaluations of treatments for patients with epilepsy. As well as looking at both pharmacological (anti-epileptic drugs) and non-pharmacological (e.g. epilepsy surgery, ketogenic diet, vagus nerve stimulation) therapies, this review examines the methodological quality of the full economic evaluations included. A total of 40 publications were included in this review. In general studies had an acceptable quality. There was, however, a substantial difference in methodology in all included articles which hampered the attempt to combine information meaningfully. Heterogeneity between the studies stresses the need to define a reference case.

Chapter 8 describes an RCT to examine long-term retention rate, clinical outcomes, cost-utility and cost-effectiveness of the Ketogenic Diet (KD) compared with care as usual (CAU) in children and adolescents with intractable epilepsy from a societal perspective. The KD group resulted in more responders and showed greater improvement on seizure severity. Furthermore, the KD did not lead to worsening of side-effects other than gastrointestinal problems (only at 4 months' follow-up). However, as only a minimal difference in QALYs was found between the KD group and the CAU group, the resulting cost per QALY ratios were inconclusive.

Chapter 9 describes the study design of the so-called ZMILE-study (Zelf-management Interventie Leven met Epilepsie). A multi-component self-management intervention (MCI) which combines a self-management/education program with e-Health interventions was developed for adults with epilepsy. The overall objective of this study was to assess the (cost)-effectiveness and feasibility of the $\mathrm{MCl}$, aiming to improve self-efficacy and concordance in patients with epilepsy.

Chapter 10 reports on the results of the economic evaluations of the ZMILE-study. Objective of this economic evaluation was to compare the cost-effectiveness of the $\mathrm{MCl}$ compared to care as usual (CAU) in adult patients with epilepsy. The $\mathrm{MCl}$ did not lead to significantly higher levels of self-efficacy among adults with epilepsy. Furthermore, EQ-5D$5 \mathrm{~L}$ score did not differ between the intervention group and the control group. A (short-term) effect was found in estimates derived from the QOLIE-3 1 p, but disappeared after baseline correction. Although there was no statistically significant difference found after baseline adjustments, cost-effectiveness estimates appear to be promising.

Chapter 11 discusses the main conclusions and answers to the research questions, with some elaboration, followed by a more detailed discussion of preference studies, selfmanagement and economic evaluations in epilepsy. Directions for further research are also indicated. It is recommended, among other things, to obtain greater insight into measurement of quality of life in epilepsy, on methodologies which can be used to integrate patients', physicians', and decision-makers' preferences in resource allocation decisions, a more standardized approach to conducting economic evaluations within the field of epilepsy, to include HTA in medical education, and to always perform an economic evaluation when conducting a clinical trial. 



\section{Samenvatting}
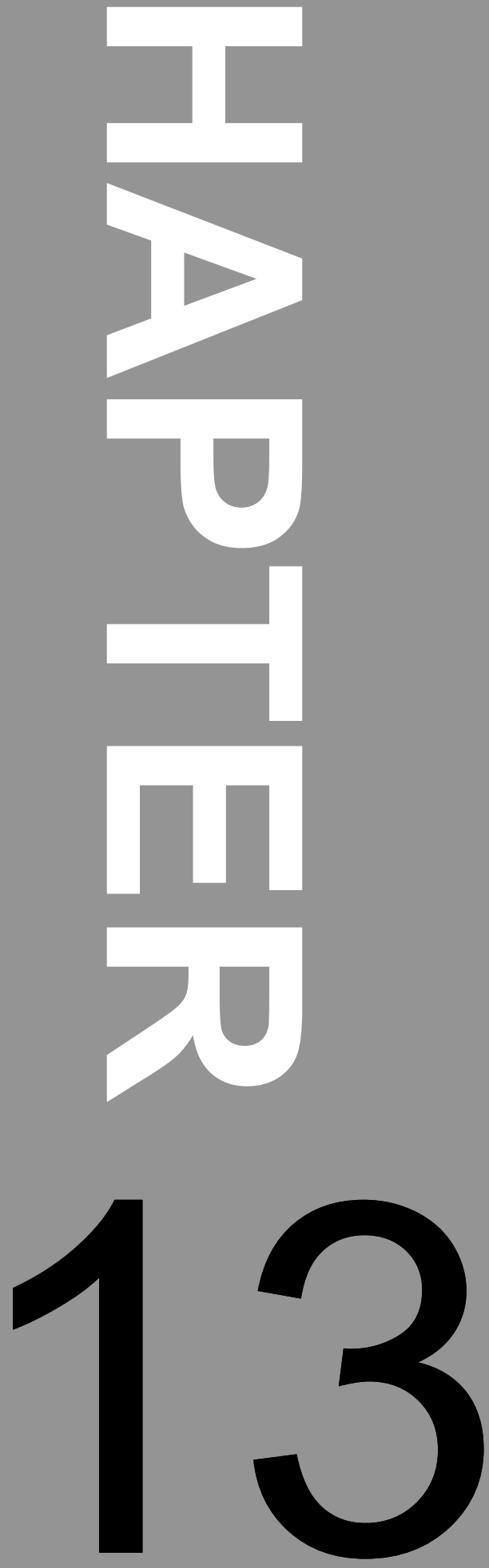



\section{Samenvatting}

Health Technology Assessment (HTA) is een multidisciplinair vakgebied waarin, onder andere, kosten, kosteneffectiviteit en voorkeurenonderzoek een belangrijke rol spelen. Gelet op de stijgende kosten voor de gezondheidszorg van de afgelopen decennia, is er een belangrijke rol weggelegd voor economische evaluaties en de bijbehorende kosteneffectiviteitscriteria. Daarnaast is de kijk op de traditionele relatie tussen patiënt en behandelaar de afgelopen decennia veranderd en is de nadruk op de autonomie van de patiënt komen te liggen. $\mathrm{Er}$ is een bewustzijn voor verschillen in keuzes die gemaakt worden tussen patiënten en behandelaars. Dit vraagt om een meer patiëntgerichte en efficiënter gezondheidszorgstelsel.

Deze thesis beschrifft methodologische aspecten en voorkeurenstudies in HTA binnen epilepsie. Daarnaast wordt er gekeken naar de kwaliteit en kwantiteit van economische evaluaties binnen epilepsie. Als laatste wordt de kosteneffectiviteit onderzocht van twee behandelingen voor mensen met epilepsie: een studie naar de kosteneffectiviteit van het ketogeen dieet bij kinderen met refractaire epilepsie en een studie naar de kosteneffectiviteit van een zelfmanagement interventie voor volwassenen met epilepsie.

Hoofdstuk 1 is een inleidend hoofdstuk waarin achtergrondinformatie wordt gegeven over epilepsie en waarin belangrijke begrippen binnen HTA worden toegelicht. Epilepsie is een neurologische aandoening gekarakteriseerd door terugkerende plotselinge aanvallen veroorzaakt door elektrische ontladingen in de hersenen.

Ongeveer $30 \%$ van de patiënten met epilepsie is niet aanvalsvrij terwij ze antiepileptica slikken. Deze patiënten, met oncontroleerbare epileptische aanvallen en waarbij twee of meer ani-epileptica niet aanslaan, worden ook wel refractaire epilepsie patiënten genoemd. Epilepsie heeft een grote impact op de kwaliteit van leven van patiënten, hun omgeving, en de samenleving als geheel.

Het doel van HTA is het informeren van beleidsmakers en clinici met behulp van evidence-based informatie -over gezondheidsinterventies en patiëntenzorg. In deze thesis staan twee HTA onderwerpen centraal, namelijk (patiënten)voorkeurenonderzoek en economische evaluaties. Voorkeurenstudies proberen voorkeuren met betrekking tot een bepaald onderwerp (bijv. een nieuw medicijn) van vooraf gespecificeerde belanghebbenden (bijv. patiënten) te identificeren en te prioriteren. Deze voorkeuren worden meestal verkregen door middel van stated preference methoden, zoals een discrete choice experiment, best-worst scaling of time trade-off opdrachten.

Economische evaluatiestudies vergelijken zowel de kosten als de effecten van twee (of meer) alternatieve interventies of behandelingen met elkaar op een systematische manier. Vervolgens wordt aan de hand van deze gegevens de incrementele kosteneffectiviteitsratio berekend.

Aan het eind van dit hoofdstuk worden de onderzoeksvragen die als uitgangspunten voor deze thesis zijn gebruikt toegelicht. 
Hoofdstuk 2 beschrifft de resultaten van een discrete choice experiment waarin werd gekeken 1) in hoeverre bepaalde karakteristieken van procedures die gebruikt worden bij de diagnose van epilepsie de voorkeur van patiënten voor die procedures beïnvloedt; 2) naar het relatieve belang van deze karakteristieken; en 3) naar het berekenen van een hypothetische utiliteitsscore voor electro-encefalografie (EEG) en magneto-encefalografie (MEG) op basis van de uitkomsten van het onderzoek. Respondenten werd gevraagd om een aantal binaire keuzes te maken tussen (hypothetische) diagnostische procedures voor epilepsie, bestaande uit een aantal karakteristieken met elk variërende niveaus (bijv. $30 \mathrm{~min}$ reistijd vs. $60 \mathrm{~min}$ reistijd). Uit dit onderzoek bleek dat MEG niet patiëntvriendelijker is dan EEG. Aanvullend onderzoek liet zien dat respondenten een sterke voorkeur hedden voor lange termijn 24-uurs EEG-registratie in vergelijking met EEG na slaapdeprivatie.

Hoofdstuk 3 rapporteert de resultaten van een time trade-off (TTO) studie waarin utiliteiten werden afgeleid voor epilepsie-gerelateerde gezondheidstoestanden. In deze studie werden gezondheidstoestanden gebaseerd op epilepsie-gerelateerde karakteristieken (bijv. aantal aanvallen, aanvalsernst, bijwerkingen van medicijnen) gewaardeerd door een steekproef uit de algemene Nederlandse bevolking ( $N=525)$ Deze studie biedt een utiliteitsfunctie voor het transformeren van klinisch relevante uitkomstmaten binnen de epilepsie naar utiliteiten. Daarnaast bleek vit deze studie dat aanvalsernst een grotere impact had op kwaliteit van leven dan aanvalsfrequentie en bijwerkingen van medicijnen.

Hoofdstuk 4 beschrijft een studie waarin de responsiviteit van en de correlatie tussen de EQ-5D-5L en de QOLIE-31P in patiënten met epilepsie is onderzocht. De EQ-5D-5L vertoonde in hoge mate ceilling effects en kleine effects sizes (EFs) en standardized response means (SRMs). De QOLIE-31P daarentegen, liet geen ceilling effects zien en had kleine tot matige EFs en SRMs. Het opstellen van een mapping functie waarbii de waardes van de QOLIE-3 1 P middels een regressieformule werden vertaald naar EQ-5D$5 \mathrm{~L}$ waardes was slecht beperkt mogelijk doordat de modellen niet goed aansloten bij de vergaarde gegevens, wat resulteerde in lage aangepaste $R^{2}$ waardes. De resultaten uit deze studie benadrukken het belang van de ontwikkeling van een conditie-specifiek, op voorkeuren gebaseerd meetinstrument dat kan worden gebruikt binnen het QALYframework. De verkregen uitkomsten van een dergelijk meetinstrument kunnen op deze manier worden toegevoegd als aanvulling op de standaard economische evaluatie.

Hoofdstuk 5 presenteert een systematische review van de huidige praktijk en trends in het gebruik van best-worst scaling (BWS) binnen de gezondheidszorg. Het doel van deze review was om BWS studies in de gezondheidszorg te identificeren, de kwaliteit kritisch te beoordelen en trends ten aanzien van belangrijke aspecten van BWS in de tijd te identificeren.. In totaal zijn 62 studies geïdentificeerd waarvan 26 BWS object case studies, 29 BWS profile case studies en 7 BWS multi-profile case studies. Het gebruik van 
BWS in gezondheidszorg is drastisch gestegen de afgelopen jaren. Ongeveer tweederde van de geïdentificeerde studies was uitgevoerd in de afgelopen twee jaar. Recente studies hadden veelal kleinere steekproefgroottes, bestonden uit minder attributen en gebruikte minder ingewikkelde analytische methoden dan oudere studies. De kwaliteit van de BWS studies was over het algemeen acceptabel.

Hoofdstuk 6 geeft handvatten voor het uitvoeren van een systematisch literatuuronderzoek naar economische evaluaties om vergoedingsbeslissingen binnen de gezondheidszorg te nemen. Het specifieke artikel in deze thesis is onderdeel van een serie van drie papers en gaat in op de data extractie, het risico op bias en de mogelijkheid om resultaten te vertalen naar een andere setting (transferability). In dit artikel worden aanbevelingen gedaan voor checklist die gebruikt kunnen worden bij de risk of bias en transferability beoordeling. Deze aanbevelingen zijn gebaseerd op bestaande, toonaangevende internationale checklists waarbij deze zijn beoordeeld op haalbaarheid, kwaliteit en de manier van ontwikkeling van de vragenlijst.

Hoofdstuk 7 betreft een systematische literatuurstudie naar economische evaluaties van behandelingen voor mensen met epilepsie. In deze studie zijn zowel farmacologische (anti-epileptica) als niet farmacologische interventies (bijv. epilepsie chirurgie, ketogeen dieet, nervus vagus stimulator) onderzocht. Het doel van de review was om de beschikbaarheid en methodologische kwaliteit van de geïdentificeerde studies te beoordelen. Over het algemeen waren studies van hoge kwaliteit. Echter, er zaten substantiële methodologische verschillen tussen de geïdentificeerde studies waardoor het niet mogelijk was om resultaten van studies te combineren. Deze heterogeniteit tussen de verschillende studies benadrukt de behoefte aan een meer gestandaardiseerde aanpak van economische evaluaties binnen epilepsie.

Hoofdstuk 8 beschrijft de resultaten van een gerandomiseerde gecontroleerde studie naar de langetermijneffecten, retentiegraad, kostenutiliteit en kosteneffectiviteit van het ketogeen dieet (KD) vergeleken met de gebruikelijke zorg in kinderen en adolescenten met epilepsie vanuit een maatschappelijk perspectief. In de KD groep waren meer patiënten met een succesvolle behandeling en ook lieten meer patiënten een afname zien in hun aanvalsernst. Naast bijwerkingen met betrekking tot het maagdarmstelsel (alleen op 4 maanden follow-up) zorgde het KD niet voor extra bijwerkingen. Echter, er werd een minimaal verschil gevonden tussen beide groepen in kwaliteit van leven. Dit resulteerde in ongunstige kosteneffectiviteitsratio's.

Hoofdstuk 9 beschrijft de resultaten van het studiedesign van de ZMILE studie (Zelfmanagement Interventie Leven met Epilepsie). In deze studie is een interventie bestaande uit meerdere componenten, zoals voorlichting en e-health hulpmiddelen, ontwikkeld met als doel het stimuleren van zelfmanagement voor volwassenen met epilepsie. Het doel van deze studie was om de kosteneffectiviteit en haalbaarheid van 
deze interventie te beoordeelden, met als primaire vitkomstmaten zelfredzaamheid en concordantie in patiënten met epilepsie.

Hoofdstuk 10 geeft de resultaten van de economische evaluatie van de ZMILE-studie weer. Het doel van deze economische evaluaties was om de kosteneffectiviteit van de ZMILE interventie te bepalen in vergelijking met de gebruikelijke zorg voor volwassenen met epilepsie. De interventie groep had geen significant hogere zelfredzaamheid en ook de kwaliteit van leven was niet statistisch significant tussen beide groepen. Echter, hoewel er geen significante verschillen tussen beide groepen te vinden was, lijken de kosteneffectiviteitsschattingen toch veelbelovend.

Hoofdstuk 11 bediscussieert de voornaamste conclusies uit deze thesis en beantwoord de diverse onderzoeksvragen die aan het begin van deze thesis zijn opgesteld. Vervolgens volgt een meer gedetailleerde discussie over voorkeurenonderzoek, zelfmanagement, en economische evaluaties in epilepsie. Ook worden er aanbevelingen gedaan voor verder onderzoek. Het wordt, onder andere, aanbevolen 1) om meer inzicht te verkrijgen in het meten van kwaliteit van leven binnen epilepsie; 2) om meer inzicht te krijgen in het integreren van de voorkeuren van patiënten, clinici en beleidsmakers in vergoedingsbeslissingen; 3) het creëren van een standaardprotocol voor het vitvoeren van economische evaluaties binnen epilepsie; 4) het includeren van HTA binnen medische (vervolg)opleidingen; en 4) om altijd een economische evaluatie vit te voeren wanneer met een klinische studie vitvoert. 
Addendum
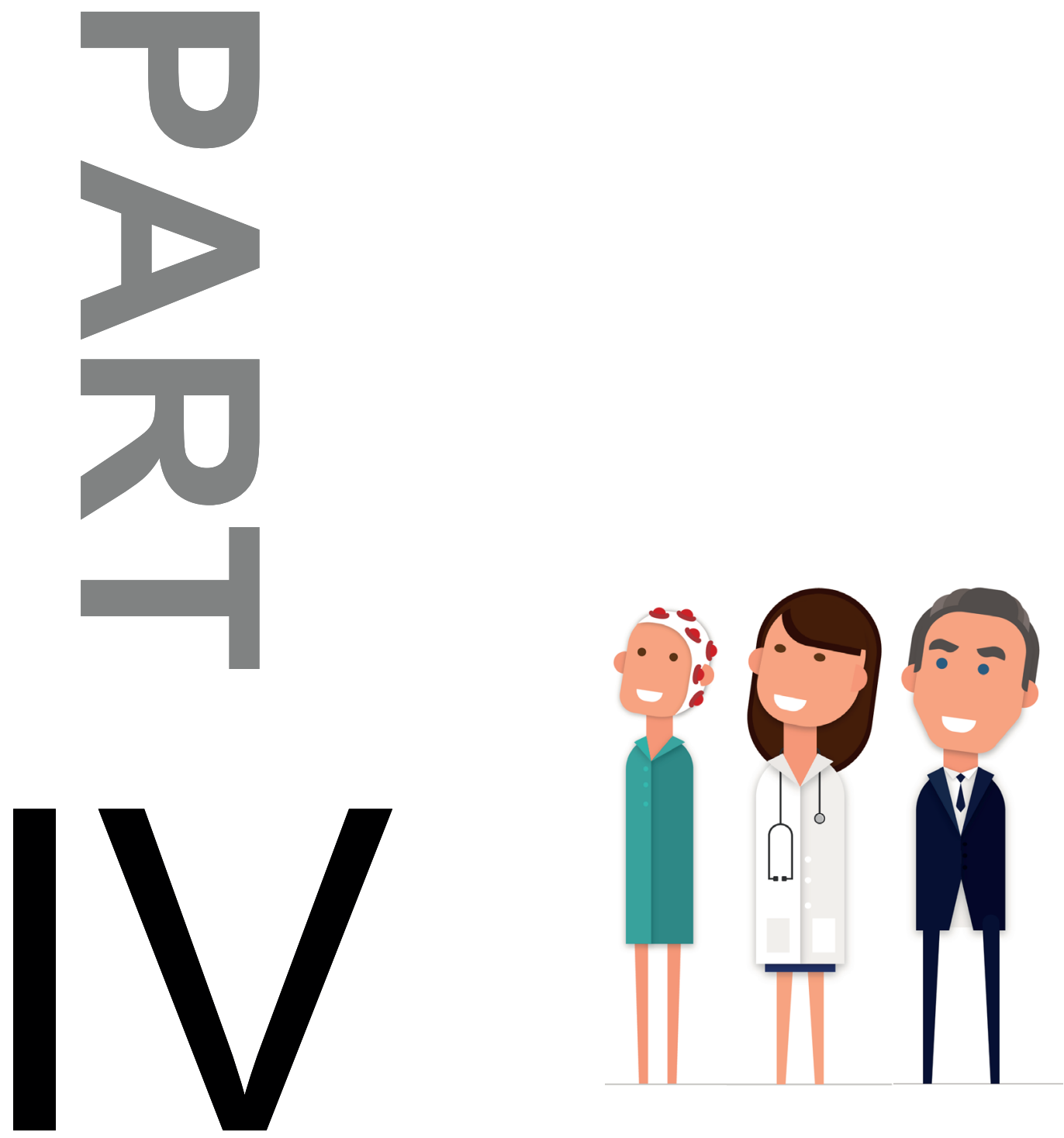

Valorization 



\section{Valorization}

Epilepsy is the fourth most common neurological disorder and affects people of all ages. In the Netherlands, in 2015, roughly 180,000 were suffering from epilepsy, with 14,000 newly identified cases. Epilepsy results in various clinical manifestations, and seizure severity and frequency vary per person. In 2011, in The Netherlands, costs of epilepsy were about 248.7 million euro, that is $4.8 \%$ of the total healthcare budget spend on neurological disorders. The rising healthcare costs attributable to epilepsy in combination with an overall increase in healthcare expenditure nationwide and a constraint healthcare budget stress the need for economic evaluations with the ultimate goal to achieve an efficient allocation of resources.

To achieve a more efficient and patient-centered healthcare system, scientific research is only the first step. The second step is to reach out to various stakeholders within the healthcare sector to disseminate the results of scientific research. Scientific knowledge should benefit society as a whole and it is important to translate the value of scientific work into ideas and solutions societies face. As defined in the $\mathrm{PhD}$ regulations of Maastricht University in 2013, the process of valorization is "the process of value creation from scientific knowledge by making it available for societal (or economic) use and make it suitable for translation to competitive products, services, or processes". Hence, this chapter provides an overview of the relevance of this thesis for the most important stakeholders and a description of the dissemination of the findings of this thesis.

\section{Relevance for researchers}

The studies described in this thesis are relevant for researchers in the field of health technology assessment (HTA). Chapter two and three provide examples of application of a discrete choice experiment and a time trade-off study in epilepsy. Chapter four provides an overview of best-worst scaling in healthcare, which may provide important understandings and insight in to the usefulness of this technique.

For researchers focusing on economic evaluations, especially in epilepsy, the performance of the EuroQol-5D-5L in adults with epilepsy described in chapter five is important as it makes them aware of the shortcomings of this instrument. As this instrument is recommended in the national guidelines for conducting economic evaluations, it is important to know what its limitations are when using the instrument. For example, these results may give rise to the development of another generic or condition-specific instrument to examine quality of life in patients with epilepsy or these results may highlight the need for additional questionnaires besides the EuroQol-5D-5L when conducting an economic evaluation in epilepsy.

This thesis also provides an overview of the current economic evaluations conducted in epilepsy, which indirectly highlights potential knowledge gaps. This may guide future researchers in developing (trial-based) economic evaluations to close these gaps. In addition, the heterogeneous methodology may give rise to the development of a reference 
case for the conduct of economic evaluations in epilepsy. Lastly the two trial-based economic evaluations in which the ketogenic diet (KD) and a multi component selfmanagement intervention are being compared to care as usual add to the current scientific knowledge of both the KD and self-management in epilepsy.

\section{Relevance for patients and healthcare professionals}

Some of the more methodological studies described in this thesis are not directly relevant for patients and healthcare professionals. However, on the long run, patients and healthcare professionals indirectly benefit from improved scientific methods as it enables researchers to perform high quality studies, resulting in results with greater certainty.

For patients and healthcare professionals, it is relevant to know what the (costleffectiveness is of interventions aiming to treat epilepsy is. Although it is unrealistic to assume that every patient or and healthcare professional is able to understand the studies described in this thesis in detail, the overall conclusion should be presented in a comprehensible way. It is important for patients and healthcare professionals to be aware of alternative treatments as $30 \%$ of the people with epilepsy is still not seizure free while being on anti-epileptic drugs. For example, although the retention rate of the KD is rather low, the KD was shown to be of significant importance to those who were still on the diet at the end of the study. The same holds true for the multi component self-management intervention, which (marginally) improved patients' health status.

\section{Relevance for policy makers or healthcare insurance companies}

For policy makers or healthcare insurance companies it is important to look at the (costleffectiveness of treatments in order to make a deliberate choice on what to reimburse. The ZMILE-study demonstrated favourable cost-effectiveness ratios, which may create a positive attitude towards self-management interventions in general, but more specifically the ZMILE intervention for adults with epilepsy. Regarding the KD, although unfavourable cost-effectiveness ratios were found, the KD was shown to be effective on the short term and was demonstrated to be effective for those who were still on the diet at the end of the follow-up period. The low retention rate may make policy makers or healthcare insurance companies willing to investigate the most optimal way of delivering the KD and which patients are best able to benefit from the KD.

\section{Dissemination}

To stimulate the dissemination among fellow researchers, it is important that the results of all scientific studies described in this thesis and the studies which are not included in this thesis, are being published in international journals. Of the nine articles described in this thesis, eight have been published so far and the remaining two are currently under review for publication in international journals. In addition, the plan is to write and publish a Dutch translation of the economic evaluation of the ZMILE study in the Dutch journal "Epilepsie, 
periodiek voor professionals" to reach out to Dutch neurologists. A publication regarding the short term effects of the KD is already available in this journal. Besides this, a general (Dutch) introduction to economic evaluations in epilepsy was written (and published) in this same journal.

Besides (inter)national publications, results of the studies described in this thesis have been presented at (inter)national conferences such as conferences of the International Society of Pharmacoeconomic Outcomes and Research (ISPOR), the Lowlands Health Economics Study Group (LolaHESG), the annual CAPHRI day, the annual International Clinical Symposium of Kempenhaeghe, and a congress of the European Academy of Neurology. In addition, all researchers at epilepsy center Kemenhaeghe are required to present their results (or progress) twice a year for interested staff members of Kempenhaeghe and specially invited (inter)national experts in the field of epilepsy.

To enhance the dissemination and ease of use of the KD, a website has made available for people with epilepsy who are currently on the KD. This website contains information regarding the KD and provides daily recipes for the three different types of the KD, namely the modified Atkins diet, the MCT-diet, and the classic KD. These recipes are developed in consultation with a master chef and makes life easier for those on the KD. The menus can be found at www.ketogeenmenu.nl. In order to make patients and their parents/caretakers get used to the new meals, workshops were provided. Furthermore, when the patients give their permission, their treating physician and dietician can view their personal data. With this, the dietician and/or treating physician can anticipate on experienced side-effects or the level of ketosis by changing the diet. The development of this new website is sponsored by fonds NutsOhra, CZFonds, Epilepsiefonds and ZonMw.

Regarding the multi-component self-management intervention ( $\mathrm{MCl}$; the ZMILE-study), with valorization in mind, an advisory group was composed already in the designing phase of the study. This advisory group consisted of a patient representative, and people from healthcare insurances, and the industry. The advisory group was consulted/informed four times a year and the final results of the ZMILE-study were presented to them. As the ZMILE-study has been completed quite recently, we are now working on the implementation of the intervention. Fortunately, the first conversations regarding implementation of the $\mathrm{MCl}$ are promising and we hope to be able to offer patients the $\mathrm{MCl}$ at the Academic Center for Epileptology in Maastricht in the near future. In addition, a process evaluation was carried out (not described in this thesis), to evaluate patients' view on the $\mathrm{MCl}$ and to identify potential barriers or facilitators for future implementation (such as the availability of the education adherence monitoring system and minor adjustments to the intervention protocol). 



\section{List of publications}





\section{List of publications}

\section{Peer-reviewed international publications}

Wijnen BFM, Leenen LAM, de Kinderen RJA, van Heugten CM, Evers SMAA, Majoie MHJM: An economic evaluation of a multi-component intervention for adults with rpilepsy (ZMILE study). Epilepsia: in press

Cheung KL, Wijnen BFM, de Vries H. 2017. Online smoking cessation interventions in the Netherlands: effects, cost-effectiveness, and theoretical underpinnings. J Med Internet Res; in press

Wijnen BFM, de Kinderen RJA, Lambrechts DAJE, Postulart D, Aldenkamp BAP, Majoie MHJM, Evers SMAA. Long-term clinical outcomes and economic evaluation of the ketogenic diet versus care as usual among children and adolescents with intractable epilepsy. Epilepsy Res. 2017;132:91-9.

Wijnen BFM, van Mastrigt GAPG, Evers SMAA, Gershuni O, Lambrechts DAJE, Majoie MHJM, Postulart D, Aldenkamp BAP, de Kinderen RJA. A systematic review of economic evaluations of treatments for patients with epilepsy. Epilepsia. 2017.

Wijnen BFM, Van Mastrigt G, Redekop WK, Majoie H, de Kinderen R, Evers S. (2016). How to prepare a systematic review of economic evaluations for informing evidencebased healthcare decisions: data extraction, risk of bias, and transferability (part 3/3). Expert Rev Pharmacoecon Outcomes Res. 2016; 16(6):723-732.

van Duin MJ, Conde R, Wijnen BFM, Evers SM, Gonzalez-Rodriguez JL, Govers MJ, Hiligsmann M. The impact of comorbidities on costs, utilities and health-related quality of life among HIV patients in a clinical setting in Bogota. Expert Rev Pharmacoecon Outcomes Res. 2016.

Leenen LA, Wijnen BFM, de Kinderen RJ, van Heugten CM, Evers SM, Majoie MH. Are people with epilepsy using eHealth-tools? Epilepsy Behav, 2016;64(Pt A):268-272.

Wijnen BFM, Cheung KL, Hollin IL, Janssen EM, Bridges JF, Evers SMAA, Hiligsmann M. (2016). Using best-worst scaling to investigate preferences in health care. PharmacoEconomics. 2016;34(12):1195-1209.

de Kinderen RJA, Wijnen BFM, van Breukelen G, Postulart D, Majoie HJM, Aldenkamp AP, Evers SMAA. From clinically relevant outcome measures to quality of life in epilepsy: a time trade-off study. Epilepsy Res. 2016. 
de Kinderen RJ, Lambrechts DA, Wijnen BFM, Postulart D, Aldenkamp AP, Majoie MH, Evers SM. An economic evaluation of the ketogenic diet versus care as usual in children and adolescents with intractable epilepsy: An interim analysis. Epilepsia. 2016;57(1):4150 .

Hop MJ, Wiinen BFM, Nieuwenhuis MK, Dokter J, Middelkoop E, Polinder S, van Baar $M E$, Dutch Burn Repository group. Economic burden of burn injuries in the Netherlands: A 3 months follow-up study. Injury. 2016;47(1):203-210.

Wijnen BFM, van der Putten IM, Groothuis $S$, de Kinderen RJA, Noben CYG, Paulus ATG, Ramaekers BLT, Vogel GCWM, Hiligsmann M: Discrete-choice experiment versus rating scale exercise to evaluate the relative importance of attributes: a study of the Maastricht ISPOR Student Chapter. Expert Rev Pharmacoecon Outcomes Res. 2015;3: $1-8$.

Wijnen BFM, de Kinderen RJA, Colon AJ, Dirksen CD, Essers BAB, Hiligsmann M, Leijten FSS, Ossenblok PPW, Evers SMAA. Eliciting patients' preferences for epilepsy diagnostics: A discrete choice experiment. Epilepsy Behav. 2014;31:102-109.

Wijnen B.F.M., Leenen L, de Kinderen R, Majoie M, van Heugten CM, Evers S: (Cost)effectiveness of a multi-component intervention for adults with epilepsy: study protocol of a Dutch randomized controlled trial (ZMILE study). BMC Neurol 2014;14:255

\section{Dutch papers/book chapters}

Wijnen BFM, van Gils P, de Kinderen RJA, Evers SMAA. Tijd voor uniformiteit tussenmaatschappelijke kostenbatenanalyses en economische evaluaties in de zorg. Tijdschr gezondheidswet. 2017.

Wijnen BFM, de Kinderen R, Hiligsmann M, Evers SMAA. "Economic evaluations within multiple sclerosis" written as a contribution to the book "Multiple sclerosis and clinical scientific methods" published in January 2016 (chapter in Dutch only). ISBN 9789057611315. Merck B.V. Book chapter.

Wijnen BFM, Majoie M, Van Heugten C, de Kinderen R, Leenen L, Evers S. Economische Evaluatiestudies Binnen de Epilepsie. Epilepsie periodiek voor professionals, 2015;2(13):16-20.

\section{Reports}

de Kinderen RJA, Wijnen BFM, Evers SMAA, Hiligsmann M, Paulus ATG, de Wit GA, van Gils PF, Over EAB, Suijkerbuijk AWM, Smit F. Social cost-benefit analysis of tobacco control policies in the Netherlands. Maastricht University, RIVM \& Trimbos Institute. 2016. 
Cheung KL, Wijnen BFM, de Vries, $\mathrm{H}$ Online smoking cessation interventions in the Netherlands. A project of MIRO - innovation in smoking cessation. 2016.

\section{Submitted}

Wijnen BFM, Mosweu I, Majoie MHJM, Ridsdale L, de Kinderen RJA, Evers SMAA, McCrone P. Mapping of the QOLIE-31P to EQ-5D-5L Utilities and Comparison of the Responsiveness of both Instruments in Epilepsy. Submitted to European Journal of Health Economics

Cheung KL, Wijnen BFM, Hiligsmann M, Coyle K, Coyle D, Pokhrel S, de Vries H, Präger $M$, Evers SMAA. The cost-effectiveness of internet-based interventions for smoking cessation in the Netherlands. An analysis based on the EQUIPT ROI model. Submitted to Addiction.

Goei H, Wijnen BFM, Mans S, de Jongh MA, van der Vlies $\mathrm{CH}$, Polinder S, van Loey NE, van Baar ME. Epidemiology, costs and quality of life of burn injuries presenting to emergency departments; a pilot study. Submitted to Burns \& Trauma.

Hendriks A, Wijnen BFM, van Engelen R, Conde R, Evers SMAA, Gonzalez J, Govers M, Mühlbacher A, Hiligsmann M. A best-worst scaling to elicit patients' preferences for HIV/AIDS treatment in Colombia. Submitted to AIDS Education and Prevention.

Leenen LAM, Wijnen BFM, van Haastregt JCM, de Kinderen RJA, Evers SMAA, Majoie $M H J M$, van Heugten CM. Process evaluation of a multi-component self-management intervention for adults with epilepsy (ZMILE study). Submited to Epilepsy \& Behavior.

\section{Abstracts}

de Kinderen RJA, Wijnen BFM, Postulart D, Majoie MHJM, Aldenkamp AP, Evers SMAA. From clinically relevant outcome measures to quality of life in epilepsy. Value Health. 2014;17(7):A573.

Hop JM, Wijnen BFM, Nieuwenhuis M, Dokter J, Middelkoop E, Polinder S, van Baar M. Economic Burden of Burn Injuries in the Netherlands. Value Health. 2014;17(7):A606A607.

Wijnen BFM, Leenen LAM, de Kinderen RJA, Majoie $M H$, van Heugten CM, Evers SMAA. (Cost-) Effectiveness of A Multi-Component Intervention for Adults With Epilepsy: Study Protocol of A Dutch Randomized Controlled Trial. Value Health. 2014;17(7):A582A583. 
Wijnen BFM, van der Putten IM, Groothuis $S$, de Kinderen RJA, Noben CYG, Paulus ATG, Ramaekers BLT, Vogel GCWM, Hiligsmann M. Discrete-choice experiment versus rating scale exercise to evaluate the relative importance of attributes: a study of the Maastricht ISPOR Student Chapter. Value Health. 2014;17(7):A568.

Krueger L, Tamminga J, Wijnen BFM, Hiligsmann M, Evers SMAA. An European overview of the future changes in evidence requirements for the reimbursement of orphan drugs: a stakeholder analysis value in health. In ISPOR 17th Annual International Meeting, Montreal, Canada. Value Health. 2014;17:A236.

Wijnen BFM, de Kinderen RJA, Colon AJ, Dirksen CD, Essers BAB, Hiligsmann M, Leijten FSS, Ossenblok PPW, Evers SMAA. Eliciting Patients' Preferences for Epilepsy Diagnostics: A Discrete Choice Experiment. Epilepsy Behav 2014;31:102-109. 
Dankwoord 



\section{Dankwoord}

Gefeliciteerd, je hebt zojuist het meest gelezen hoofdstuk uit deze thesis opengeslagen: Het dankwoord. Volledig terecht overigens, ik heb gedurende dit traject veel hulp en steun gehad van veel verschillende mensen. Belangrijk hierbij zijn natuurlijk alle patiënten, patiëntjes, en/of proefpersonen die hebben deelnomen aan de verschillende studies. Het is een cliché maar als jullie jezelf niet beschikbaar hadden gesteld voor wetenschappelijk onderzoek was het bijzonder beroerd afgelopen met mijn promotietraject. Enorm bedankt dat jullie dit hebben willen doen.

Daarnaast wil ik een aantal mensen in het bijzonder noemen.

Prof. dr. mr. S.M.A.A. Evers, beste Silvia, ik ben blij dat iij mijn promotor was. Vanaf mijn eerste jaar van de research master heb je mij begeleid bij mijn onderzoeksvoorstel en later ook mijn onderzoeksstage. Het voelde goed om na een kort vitstapje naar Rotterdam, weer terug te komen en mijn promotietraject onder jouw begeleiding vit te voeren. Jouw expertise, brede netwerk en vermogen om on-the-spot met oplossingen te komen is bewonderenswaardig. Daarnaast is het ook fijn dat je zo nuchter in het leven staat en makkelijk bent in de omgang. Toen ik een verkeerde vragenlijst verstuurde naar "een aantal" patiënten tijdens mijn stage heb je hier bijzonder weinig woorden aan vuilgemaakt. Je hebt me zelfs met succes overtuigd om bij Health Services Research mijn promotietraject te komen doen. Ook vond ik het fijn dat je me veel verschillende soorten projecten toevertrouwden, zoals het schrijven van een hoofdstuk of het ondersteunen van de maatschappelijke kosten-baten analyse naar tabaksontmoedigingsmaatregelen. Bedankt dat je hebt geholpen mijn onderzoekservaring zo divers te maken.

Prof. dr. H.J.M. Majoie, beste Marian, wat is het fijn om jou als promotor te hebben. Je enorme expertise, niet alleen op het gebied van epilepsie maar ondertussen ook op het gebied van health technology assessment, hebben mijn artikelen echt naar een hoger niveau gestuwd. Het is fijn dat je alles zo secuur leest en altijd tijd weet te vrij te maken om stukken te lezen, zelf met een Europese deadline voor de deur. Daarnaast ben je enorm enthousiast over de projecten waarbii je betrokken bent, wat erg motiverend werkt. Het blijft altijd leuk en verrassend om van jou een mailtje te ontvangen op een zondagavond (of nacht). Het is volledig terecht dat je gedurende mijn promotietraject professor bent geworden. Als een van je weinige mannelijke PhD studenten die je begeleidt hoop ik wel nog ooit als passagier een ritje in je Donkervoort te mogen maken!;)

Dr. R.J.A. de Kinderen, beste Reina, je was een hele goede begeleider tijdens mijn master en een hele goede co-promoter gedurende mijn promotietraject. Toen ik als "de nieuwe Reina" op Kempenhaeghe terecht kwam heb iij me wegwijs gemaakt en geholpen met alles waar ik tegenaan liep. Jij dacht altijd heel sterk aan mijn belang en wat goed was/is voor mijn ontwikkeling. Dat waardeer ik enorm. Ik heb veel van je geleerd de afgelopen 
jaren en het was altijd leuk om samen aan projecten te werken. Je bent mede verantwoordelijk voor de diversiteit in mijn onderzoekservaring.

Ik wil graag de leden van de beoordelingscommissie van dit proefschrift hartelijk danken voor het lezen en beoordelen van mij thesis: Prof. dr. Manuela Joore, Prof. dr. Paul Boon, Prof. dr. Dirk Ruwaard, dr. Joost Nicolai, en dr. Joran Lokkerbol. Dank dat u allen tijd heeft genomen om mijn proefschrift te lezen en te beoordelen.

Daarnaast wil ik iedereen bedanken die bij de vitvoering of ondersteuning van de projecten in deze thesis betrokken was. Op de eerste plaats het ZMILE-team; de verpleegkundigen, Carly Jansen, Bea Martens, Joke Creemers, Marion van Ool, Jeske Nelissen, en Peter van der Wielen, die in de avonduren bereid waren om de verschillende cursusgroepen te leiden in Maastricht, Heeze en Nijmegen. Verder wil ik de adviesgroep bedanken die betrokken was bij het gehele ZMILE traject: Arjan Wietses, Madeleine van Kan, Caroline Caron, Loes Hageman, Anouk Pieron, Bernard Vrijens, Anja Lenssen, Karin Scheele, Richard Lazeron, Francis Tan, en Christian Vader. Belangrijk was ook de PR afdeling van Kempenhaeghe, in het bijzonder Marian Verstappen, jullie hebben bijgedragen aan het behalen van het streefaantal patiënten voor de studie.

Daarnaast natuurlijk Linda voor alle logistieke ondersteuning en overige zaken waarvoor ik je kon lastigvallen. Marion, bedankt voor je tomeloze inzet voor de MEG studie.

Alhoewel ik een groot gedeelte heb gemist van de uitvoering van de KOEK-studie, wil ik iedereen bedanken die zich hiervoor heeft ingezet. De neurologen specialisten, psychologen, verpleegkundigen, en diëtisten. Ondanks de moeilijke inclusie is het een zeer mooie studie geworden.

Loes Leenen, we hebben samen een mooi project neergezet en het was leuk mijn promotietrajact parallel aan dat van jou te doen. Ik waardeer de hoeveelheid werk die je hebt verzet voor de ZMILE-studie (ookwel jouw kindje) enorm. Het was heel fijn samen te werken met iemand die alles zo secuur bijhoudt. Onze samenwerking is gelukkig nog niet ten einde want er liggen nog een paar laatste loodjes te wachten. Ik weet zeker dat je binnenkort ook jouw thesis kunt gaan indienen! Bedankt voor de gezelligheid op de kamer en de lekkere etentjes (waarvoor ook dank aan Rein viteraard).

Prof. dr. Caroline van Heugten, beste Caroline, hoewel je niet mijn directe promotor was heb ik altijd veel gehad aan je tips tijdens onze ZMILE vergaderingen. Ik vond het fijn dat je op de juiste momenten mijn artikelen hebt aangescherpt. Je leest artikelen nauwkeurig en bent zeer behulpzaam. Mijn proefschrift is een flink aantal typo's lichter door jou.

Prof. dr. Paul McCrone, thanks for being a great supervisor during my stay in London. Your expertise largely contributed to the quality of the paper. Besides that, you're also a great 
person to work with. It was great having the Christmas lunches and parties together at King's College. Hopefully we'll meet again in the future.

Dr. Danielle Lambrechts, beste Danielle, we hebben veel op en neer gemaild over de KOEK-studie. Bedankt voor je input en het nauwkeurig lezen van mijn artikelen.

Ik wil alle coauteurs bedanken die hebben bijgedragen aan mijn artikelen (in willekeurige volgorde): prof. dr. Bert Aldenkamp, dr. John Bridges, prof. dr. Gerard van Breukelen, dr. Albert Colon, prof. dr. Carmen Dirksen, dr. Brigitte Essers, prof. dr. mr. Silvia Evers, drs. Olga Gershuni, dr. Paul van Gils, prof. dr. Caroline van Heugten, drs. Ilene Hollin, drs. Ellen Janssen, dr. Reina de Kinderen, dr. Danielle Lambrechts, Loes Leenen, dr. Frans Leijten, prof. dr. Marian Majoie, dr. Ghislaine van Mastrigt, prof. dr. Paul McCrone, dr. Mickael Hiligsmann, drs. Iris Mosweu, dr. Pauly Ossenblok, dr. Debby Postulart, dr. Ken Redekop, en prof. dr. Leone Ridsdale.

Natuurlijk wil ik al mijn collega's bij HSR bedanken. In het bijzonder, Brigitte, Janet, en Suus, bedankt dat ik bij jullie terecht kon voor al mijn vragen hoe onzinnig dan ook. Ruben bedankt voor de gezellige lunches en gesprekken. Ook bedankt aan alle leden van het ISPOR student chapter en natuurlijk de medebestuursleden (Xavier, Inge, Ingrid, Sandra, Kei Long, Reina, en Sandra) voor alle mooie dingen die we hebben georganiseerd. Ghislaine, bedankt voor je hulp en input bij de totstandkoming van de epilepsie review en de "How to .."-reeks. Het heeft even geduurd (vooral de review) maar uiteindelijk zijn het mooie publicaties geworden. Ook dank aan mijn ex-collega's van het Brandwonden centrum voor de zeer leerzame periode (met name Margriet, Nicole, Helma en Anneke).

Martin (Amogre) Ayanore, thanks for being a great roommate during my PhD trajectory. With your wife and kids in Ghana, you had to go back to Ghana now and then but I really liked our conversations regarding Ghanaian politics or Ghanaian food (goat heads;)l. I still remember how surprised you were to know that we have goats as a pet.

Bobby Presly, we've only been roommates for the past few weeks as you've just started with your PhD but so far it's great to have you around in the office. It's good to have someone around who also appreciates high quality movies;) Good luck with your PhD trajectory.

Kei Long Cheung, kamergenoot van het eerste uur. Je haat dit soort dankwoorden, dus ik ga even een mooi melodramatisch stukje schrijven. Jouw kijk op de wereld is op zijn zachts gezegd bijzonder. Je discussieert over alles en vrijwel altijd kies je het standpunt van de minderheid. Ik merk dat ik hierdoor genuanceerder nadenk over bepaalde zaken. We hebben enorm veel gelachen en (teveel) geluld op de kamer maar we zijn allebei redelijk productief gebleven gedurende ons promotietraject. Je hebt de neiging om jezelf als underdog neer te zetten, maar voor iedereen die je om de tuin hebt geleid: Kei Long is de meeste excellerende van onze kamer!;) 
Gedurende mijn tijd in Maastricht (en daarvoor) heb ik het geluk gehad de nodige vriendschappen te mogen sluiten.

Pascal Rijkers en Johnny Nas, ik vind het mooi dat we al zo lang dates plannen, van stappen tot darten, bowlen, en zeevissen (haha \#mietenhut). Soms zien we elkaar een flink aantal weken niet maar het is altijd lachen als we weer iets ondernemen, mooi dat we dezelfde humor hebben (soms dan;)). De diverse vakanties/weekenden weg bevallen mij altijd goed, de meeste anekdotes zijn helaas niet geschikt voor dit hoofdstuk vrees ik (denk bijv. aan Lloret, Praag, Slantsjev Brjag). Ik hoop binnenkort weer even een mooie trip met jullie te plannen!

Studeren en wonen in een vreemde stad met mijn kookskills is spannend in het begin. Als actief lid bij eerst M.S.V. Santé en later M.S.T.V. Stennis wil ik iedereen bedanken die mijn tijd in Maastricht zo leuk maakt/heeft gemaakt. Het is fijn dat ik vanaf vrijwel het begin een leuke groep mensen heb leren kennen. Uiteraard de Heugemerboys: Frits, Jorn en Maikel. Een broederschap ontstaan op het befaamde Santé kamp. Wat begon als "wie is de chef" is vitgegroeid tot een hechte vriendschap. Wat hebben we veel feestjes/activiteiten/ weekenden ondernomen. Zelfs nu iedereen verspreidt is over het land, kan ik genieten van de keren dat we weer eens wat gaan doen. Ook schattig dat we afgelopen januari de ontgroening van Frits bii Sabaton(!) hebben mee mogen maken.

Frits, je was echt de eerste die ik kende in Maastricht (naast de drie maanden van Johnny Nas natuurlijk) en er zijn jaren geweest dat ik je echt vier keer per week zag. Mooi dat we al zo lang samen in een coverband spelen, eerst de saurusband en toen Dubbel Fout. Daarnaast hebben we precies dezelfde interesses en kunnen we echt hele dagen vollullen met slap gezever. Ik geniet van onze discussies over Fifa en een andere niet nader te noemen zeer strategische spellenreeks;).

Daaf, Frits, Joris, Marloe, en Niek, aka dé legendarische Saurusband, bedankt voor de leuke tijd. Mooi dat we afgelopen jaar een spetterend reünie optreden hebben gehouden.

Muriel, natuurlijk wil ik jou bedanken. We hebben samen veel meegemaakt en onze vriendschap gaat ook al terug vanaf dat befaamde Santé kamp. Zonder jou was ik niet waar ik nu ben en was mijn Nederlands nog steeds op een bedroevend niveau.

Dan natuurlijk alle (ex)bandleden van Dubbel Fout: Jeroen, Pieter, Twan, Frank, Paul, Bas, Frits, Maarten en Hoi Yau. Mooi dat ons oorspronkelijke plan om één optreden te doen tijdens Stensation 2012 volledig mislukt is. De optredens blijven binnen stromen. We zitten allemaal op dezelfde lijn qua ambities en visie over de band én nemen onszelf niet te serieus. Desalniettemin hebben we toch maar mooi even de BeNeLux finale van clash of the coverbands gehaald! Het is geweldig dat iedereen altijd in is voor een feestie en logeerpartijijes geen probleem zijn. Ik zou over een iedereen een anekdote willen schrijven maar dat gaat te lang worden. Bas, iij als mede-founding father van de band 
verdient wel aparte vermelding. Onze benoeming als nieuwe Sven en Jurjen van M.S.T.V. Stennis was volledig terecht en ik vind het mooi dat we zoveel dingen samen hebben gedaan en vaak bij elkaar in de commissie hebben gezeten, met als hoogtepunt natuurlijk hét stapjaar! Hopelijk kun je binnenkort je studie eindelijk eens afronden;) Fijn dat je altijd klaar staat voor anderen, waar ik de nodige keren van heb geprofiteerd. Pieter, zonder jou was ik nog steeds een schriel mannetje (lees: nóg schrieler). Je hebt me gemotiveerd om samen twee/drie keer in de week te gaan lullen in de sportschool en af en toe wat aan fitness te doen. Daarnaast is het ook buiten de sportschool altijd gezellig. Ook jii bent enorm behulpzaam en altijd bereidt te helpen als er wat is, een hele fijne eigenschap. Natuurlijk extra dank voor het fantastische figuur in hoofdstuk drie;).

Hoi Yau Chan, beste Hoi ;), ik waardeer alle dingen die je voor me doet. Het is leuk dat je altijd enthousiast bent om dingen te ondernemen en dat we veel dezelfde dingen leuk vinden. Mooi dat je ook altijd in bent voor nieuwe activiteiten en dat je me hebt geintroduceerd in de wondere wereld van de sushi! We hebben echt al een paar hele mooie reizen gemaakt (denk aan \#Fuerteventura en \#Colombia), hopelijk volgen er nog meer. Nog even knallen en dan heb je ook je research master in de pocket.

Uiteraard wil ik mijn familie bedanken, mijn ooms en tantes en neven en nichten. Mijn peetoom Ome Gerard en peettante Tante Sien (en Ome Paul). Ma, Pa en Mandy, we zijn een relatief klein gezin maar we hebben het altijd gezellig thuis. Een positieve thuissituatie is de basis geweest voor mijn carrière tot nu toe, enorm bedankt daarvoor. Mandy, trotse moeder van Bram sinds dit jaar, mooi om te zien hoe enorm goed je dit oppakt en hoeveel dingen je weet (m.b.t het verzorgen van een baby bedoel ik dan, ik ben echt een totale leek). Binnenkort hoop ik alle benodigde kennis te hebben om een eerste keer op te passen.

Ma en pa, jullie hebben ons altijd gestimuleerd om te doen wat we zelf wilden. Niet teveel regelties en down to earth. Ik ben blij dat jullie me altijd steunen als er dingen zijn waar ik mee zit of tegenaanloop. Hopelijk is het na het lezen van deze thesis ook iets duidelijker geworden wat ik de afgelopen jaren heb uitgespookt!

Ben 

About the author 



\section{About the author}

Ben Wijnen was born on the $23^{\text {rd }}$ of March 1990 in Boxtel, the Netherlands. After finishing secondary education (athenaeum) at Maurick College in Vught, he studied Health Sciences at Maastricht University, obtaining his bachelor's degree in 2011 with a major in movement sciences and a minor in bioregulation \& health. His bachelor thesis involved a study to examine the development of executive functions in children. Ben specialized in Health Technology Assessment during the Health Sciences Research master and in 2013 he obtained his master's degree

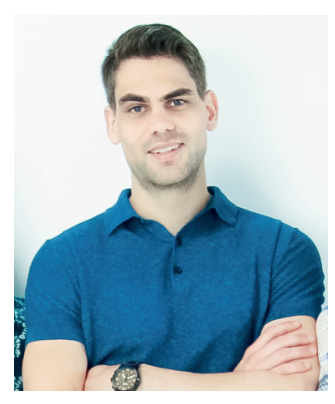
with the distinction cum laude. During his one-year internship at the Kempenhaeghe epilepsy institute, as part of the master program, he focused on patients' preferences for epilepsy diagnostics. Extracurricular activities during these years included the membership of the board of the Maastricht student tennis association ('M.S.T.V. Stennis') and playing in a (cover)band.

After his graduation he was employed as a junior researcher at the Association of Dutch Burn Centers in the Maasstad Hospital Rotterdam focusing on costs and cost-effectiveness analyses of patients admitted to the burn center. Between 2014 and 2017, Ben was a PhD candidate at the department of Health Services Research at Maastricht University and the department of Research and Development at the Kempenhaeghe epilepsy institute. His research focused on Health Technology Assessment in epilepsy, which included preference studies and economic evaluation of several interventions in the field of epilepsy such as a self-management program to improve self-efficacy, a diagnostic modality in the primary diagnosis of epilepsy, and the effects of a ketogenic diet on seizure frequency in children. In addition, he was an affiliated consultant at Synergus Inc., Stockholm were his main task was to support Stockholm's health economic team with interpretation of data and overall support. During his PhD, he was a visiting lecturer at the Universidad del Rosario of Bogota and the Universidad CES of Medellin, Colombia, and a visiting researcher at King's College London at the department King's Health Economics after receiving the Erasmus+ travel grant. During his PhD he followed courses, among others, on advanced societal cost-benefit analyses, budget impact analysis, pharmacoeconomic modelling, and acquired the university teaching qualification. Furthermore, Ben initiated and prepared research proposals and supervised several bachelor and master students. He is in the board of the ISPOR student chapter of Maastricht University which he founded and is author of several peer-reviewed (inter)nationally published articles with an impact factor (17 published). Currently, he works as a postdoctoral researcher at the department of Health Services Research, Maastricht University and the department of Research and Development at the Kempenhaeghe epilepsy institute. 


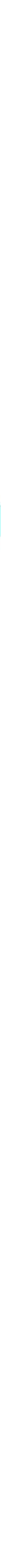

